\title{
Techno-economic and life-cycle impact analysis of solar photovoltaic microgrid systems for off-grid communities
}

By:

\section{Daniel Oluwaseun Akinyele}

A thesis submitted to the Victoria University of Wellington in fulfillment of the requirements for the degree of Doctor of Philosophy in Engineering 



\begin{abstract}
This thesis aims primarily to propose Solar Photovoltaic Microgrids (SPMs) for six different remote communities in Nigeria, one from each of the country's geopolitical zones. The research analysis is presented based on the basic load demand of 24 households within each of the selected communities. The arrangements of the houses are obtained from the community's layout provided by a building consortium.

The study first presents the intended users' basic energy needs and their daily energy usage. This information was obtained by a preliminary assessment of the users' energy consumption patterns and is then used to develop an aggregate load profile for the communities. The available solar energy resources of the different locations are carefully examined, in relation to their disparities, intermittent characteristics and seasonal variations. These factors are then used to determine suitable SPM's capacities for the users. However, because users tend to increase their energy usage over time, the research also emphasises the possibility of future load demand growth. With such consideration, more practical electrification solutions can be achieved. The study considers users' electricity demand growth of 25 to $75 \%$ of the baseline value of $175 \mathrm{kWh} / \mathrm{d}$.

The photovoltaic microgrid systems are modelled in the DIgSILENT PowerFactory environment. The lengths of the lines running from the electric power plant to the households are obtained from the community's layout. This information is included in the model, coupled with the solar energy data and the technical configurations of the PV arrays. The SPMs are modelled as $400 \mathrm{~V}, 50 \mathrm{~Hz}$ low-voltage systems, with energy supply being distributed as single-phase to the users. The study conducts load flow simulations to determine the relationship between the energy generation and the users' load demand over a 24-hour period. It also ascertains whether or not the busbar voltages and the line loadings are within an acceptable range. The simulations and the energy analysis are then extended to an 8760-hour period to obtain the microgrid system's annual electricity generation, with respect to the users' energy requirements.
\end{abstract}


The SPM system's performance is evaluated in terms of the electrical power output, energy production, normalised yields and losses, and the efficiency, according to the IEC standards 61724 and 61836. The solar energy resources' daily and seasonal variations are balanced through a battery storage system. A rigorous battery state of charge $(\mathrm{SoC})$ and reliability analysis is then used to ascertain the possible confidence level at which the proposed SPMs can meet the intended users' energy demand. The reliability indices assessed are the unmet load demand, loss of energy probability (LOEP) and the availability.

The thesis employs the Life Cycle Cost Analysis (LCCA) to evaluate the cost of the different SPMs over their useful operational lives, based on the methodology in the literature and the HOMER simulation tool. In addition, it evaluates the SPM's environmental impacts in terms of the fuel saved, $\mathrm{CO}_{2}$ emissions avoided, life-cycle emission rate and Global Warming Potential (GWP), Cumulative Energy Demand (CED), Energy Payback Time (EPBT) and the Energy Return on Investment (EROI).

The effectiveness of the proposed SPMs is evaluated by first comparing the techno-economic and environmental assessment results with those of a diesel power plant. This is also done by comparing the results with some existing related outputs in the literature, which are reported for solar photovoltaic systems in different regions of the world.

The research results indicate that it is possible to develop practical, cost-effective and reliable clean energy systems for the specified communities based on solar photovoltaic technology. The SPMs have the capability to compete with conventional electricity options - diesel/petrol generators with which some households are already familiar. Importantly, incorporating the design safety and the load growth factors in the system sizing model makes determining SPM capacities and the prediction of their annual energy production more realistic than many other existing PV energy studies in the literature. 
The performance evaluation results further indicate that different SPM sizes in a particular location have the same normalised yields and losses, and are different from the values obtained for the SPMs in other locations. These results are comparable with those reported in the International Energy Agency Photovoltaic Power System (IEA PVPS) Task 2 database for different locations in the world and other academic publications. This study reveals that the SPM with the lowest losses has the highest efficiency. This is found within a location with the lowest annual solar irradiation of all the six locations. By contrast, the SPMs in locations with relatively higher yearly solar irradiation have comparatively higher losses and lower efficiencies.

Furthermore, even though the diesel plant's initial capital cost is as low as $\sim 10-17 \%$ of those of the SPMs, its life cycle costs are $\sim 2-2.3$ times the life cycle costs of the proposed SPMs for the six locations. Over the 25year project life span, the SPMs clearly provide a significant economic benefit.

The battery average SoC probability distribution values of $>98 \%$ above the minimum set point of $30 \%$ were also achieved. The reliability indices, i.e. LOEP of $<5 \%$, availability of $>95 \%$ achieved in this study for the SPMs, are also comparable with the existing results in the literature. The SPM's estimated emission rate is $\sim 57 \mathrm{gCO}_{2} / \mathrm{kWh}$, which is lower than the values of 576 - $695 \mathrm{gCO}_{2} / \mathrm{kWh}$ obtained for diesel systems. The SPM system's GWP ranges from 3,409 to 7,945 $\mathrm{kgCO}_{2}$-eq. Also, the system's EPBTs and EROIs range from 1.11 to 1.6 years and 15.63 to 22.52 , respectively, of the specified locations.

The proposed SPM model is based on the global engineering standards and best practices and has very considerable practical applications. These can provide a reference point for governments, policymakers, researchers, designers, planners, and other stakeholders of interest in conceptualising and proceeding with the design, planning, and development of new electrification systems for remote communities. 


\section{List of Publications}

\section{Peer-Reviewed Journal Articles}

(1) Akinyele D. O, Rayudu R. K, “Life cycle impact assessment of photovoltaic power generation from crystalline silicon-based solar modules in Nigeria", Renewable Energy 2016; 101:537-549

(2) Akinyele D. O, Rayudu R. K, "Comprehensive techno-economic and environmental impact study of a localised photovoltaic power system for off-grid communities", Energy Conversion and Management 2016; 124: 266-279

(3) Akinyele D. O, Rayudu R. K, Blanchard R. "Sustainable microgrids for energy-poor communities: A spotlight of the planning dimensions", IEEE Smart Grid Newsletter July 2016.

(4) Akinyele D. O, Rayudu R. K, "Strategy for developing energy systems for remote communities: Insights to best practices and sustainability", Sustainable Energy Technologies and Assessments 2016; 16: 106-127.

(5) Akinyele D. O, Rayudu R. K, “The techno-economic and life cycle environmental performance analyses of a solar photovoltaic microgrid system for developing countries", Energy 2016; 109: 160-179

(6) Akinyele D. O, Rayudu R. K, "Community-based hybrid energy supply system: A practical and comparative approach", Applied Energy 2016; 171: 608-628.

(7) Akinyele D. O, Rayudu R. K, Rodney H. G. Tan, "Comparative study of photovoltaic technologies based on performance, cost and space requirement: Strategy for selection and application", International Journal of Green Energy 2015. DOI: 10.1080/15435075.2015.1112287

(8) Akinyele D. O, Rayudu R. K, Nair N. K. C, “Development of photovoltaic power plant for remote residential applications: The socio-technical and economic perspectives", Applied Energy 2015; 155: 131-149.

(9) Akinyele D. O, Rayudu R. K, Nair N. K. C, "Global progress in photovoltaic technologies and the scenario of solar panel plant and module performance estimation - Application in Nigeria",Renewable and Sustainable Energy Reviews 2015; 48: 112-139.

(10) Akinyele D. O, Rayudu R. K, "Review of energy storage technologies for sustainable power networks",Sustainable Energy Technologies and Assessments 2014; 8: 74-91. 


\section{Peer-Reviewed Technical Conference Papers}

(1) Akinyele D. O, Rayudu R. K, Nair N. K. C, “Grid-independent renewable energy solutions for residential use: The case of an off-grid house in Wellington, New Zealand", In Proc. IEEE/PES Asia Pacific Power and Energy Engineering Conference (APPEEC), 2015, pp. 1-5.

(2) Akinyele D. O, Rayudu R. K, Nair N. K. C, Seah W. K. G, “Clean development mechanism projects for developing countries: Potential for carbon emissions mitigation and sustainable development", In Proc. 18 $8^{\text {th }}$ National Power System Conference (NPSC), 2014, pp. 1-6.

(3) Akinyele D. O, Rayudu R. K, Chakrabarti B, Nair N. K. C, “Decentralized energy generation for end-use applications: The economic, social and environmental benefits assessment", In Proc. IEEE/PES Innovative Smart Grid Technologies (ISGT) Conference, 2014, pp. 1-4.

(4) Akinyele D. O, Rayudu R. K, "Distributed photovoltaic power generation for energy-poor households: The Nigerian perspectives", In Proc. IEEE/PES Asia Pacific Power and Energy Engineering Conference (APPEEC), 2013, pp. 1-6. 


\section{Acknowledgments}

I give glory to Almighty God for His grace upon my life throughout the Ph.D. work. Glory be to God!

I am grateful to the Victoria University of Wellington for giving me the opportunity to pursue a world-class Doctorate degree programme in Renewable Energy, through the Victoria Doctoral Scholarship that is facilitated by the Research Trust of the Victoria University of Wellington. Without this scholarship, I would not have had the opportunity to come to New Zealand to study. I also use this medium to thank my Supervisors, Dr Ramesh Rayudu, Associate Prof Nirmal Nair and Associate Prof Rodney Tan for their supervision, guidance and support, which greatly contributed to the success of the thesis. They have spent quality time throughout the research in advising and making healthy suggestions and corrections to improve the quality of the Ph.D. study, to ensure that the research is in the right and acceptable direction. Furthermore, their professional prowess has also contributed immensely to my scholarly writing, especially in journals and conference papers that I have been able to produce through this research. I am very grateful to them for their efforts and kind gestures.

Furthermore, my appreciation goes to Associate Prof Rodney Tan for his support, by giving me a copy of the FPVMPA software, which I found useful in the PV performance analysis. I also want to thank Helen Beaglehole for her great assistance in the course of my study. My colleagues within and outside the University are also appreciated, who have contributed to the success of the thesis, i.e. through their critiques, observations, and suggestions.

Finally, I want to appreciate my wife for her prayers, understanding and support throughout the Ph.D. programme. Thank you so much dear! I also thank my parents, brothers and sisters, uncles and aunties for their prayers, care, and support. May the good Lord bless all of you, in the name of Jesus Christ. 


\section{Contents}

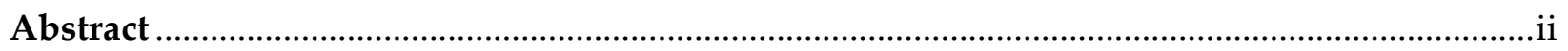

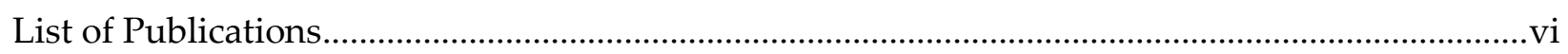

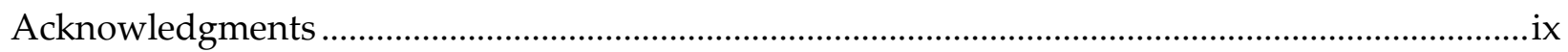

List of Acronyms and Symbols ..................................................................................................

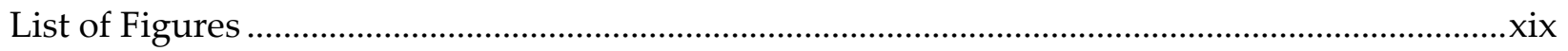

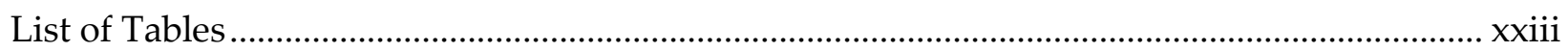

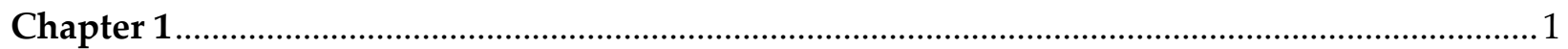

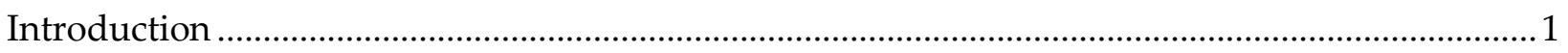

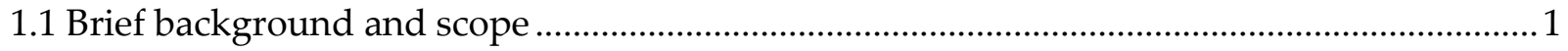

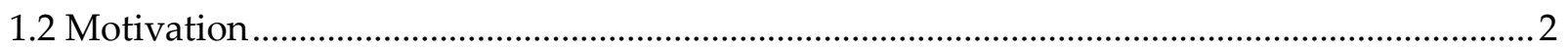

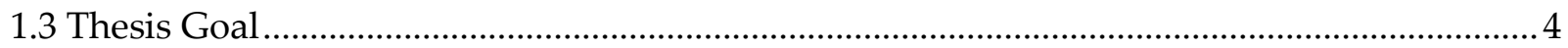

1.3.1 Research Objectives ....................................................................................................

1.4 Possible Thesis Contributions and their Usage …………………………………………...... 6

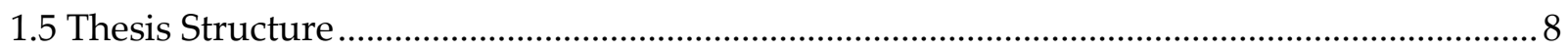

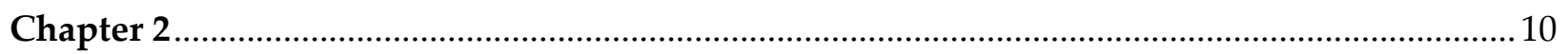

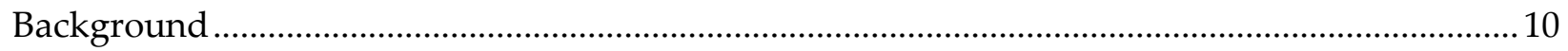

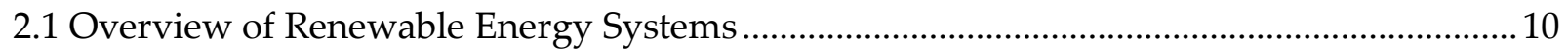

2.2 Photovoltaic technologies .................................................................................................... 12

2.2.1 Crystalline silicon (c-Si) technologies...........................................................................13

2.2.2 Thin film technologies ............................................................................................... 15

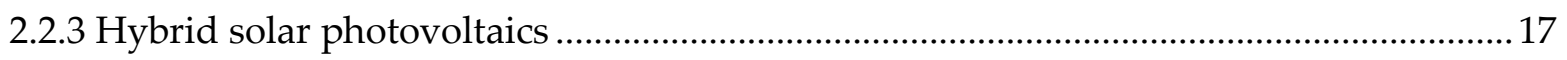

2.2.4 Organic and polymer solar photovoltaics .....................................................................18

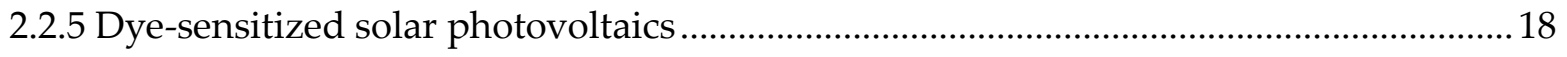

2.2.6 Nanomaterial-based solar photovoltaics ........................................................................ 18

2.3 Solar photovoltaic model ............................................................................................... 19

2.3.1 Single diode model ...................................................................................................19

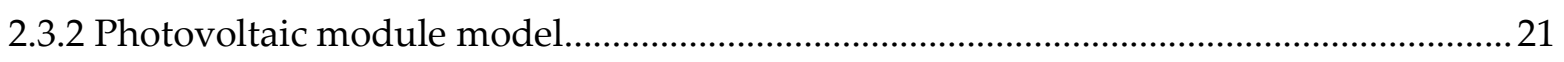




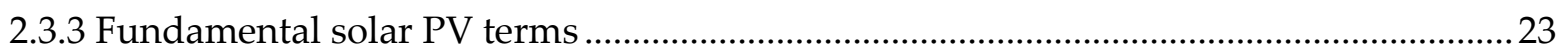

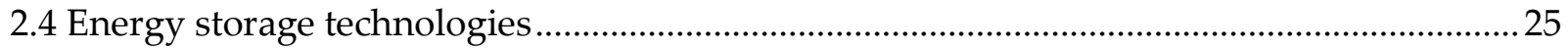

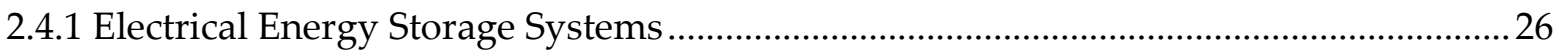

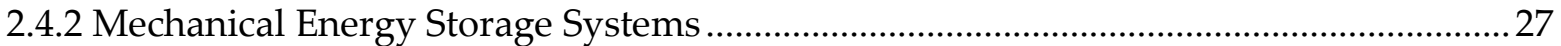

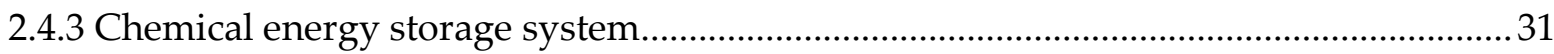

2.4.4 Thermal energy storage system …..................................................................................3

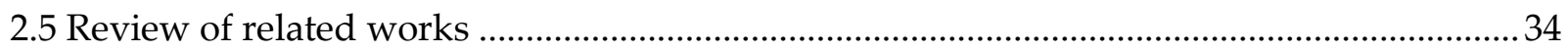

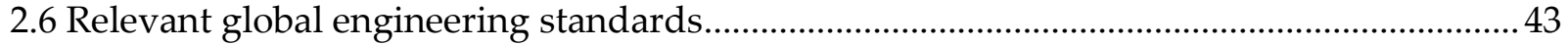

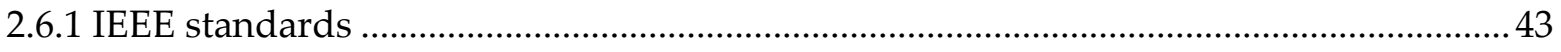

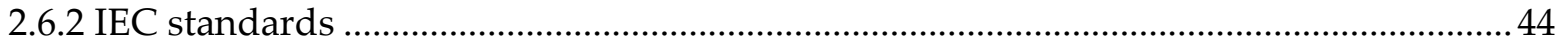

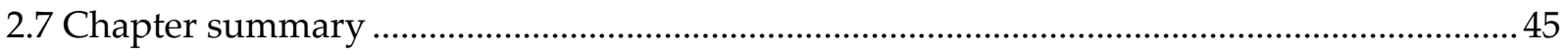

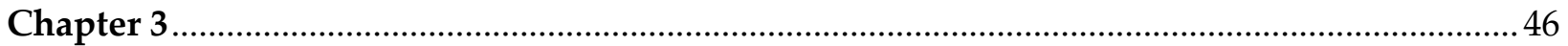

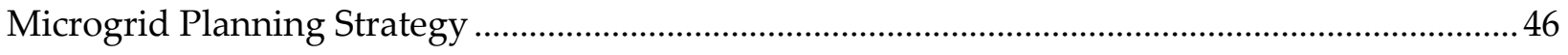

3.1 Stages of microgrid development ...................................................................................... 46

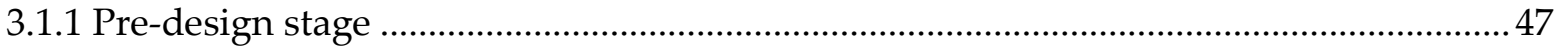

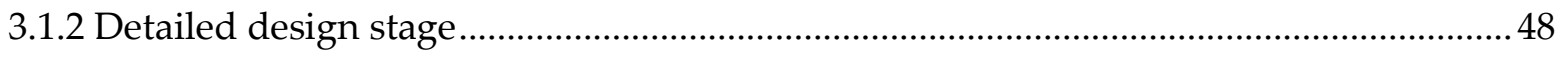

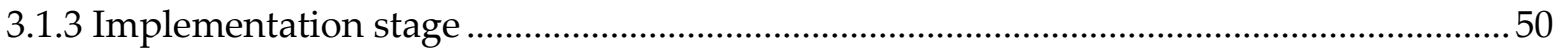

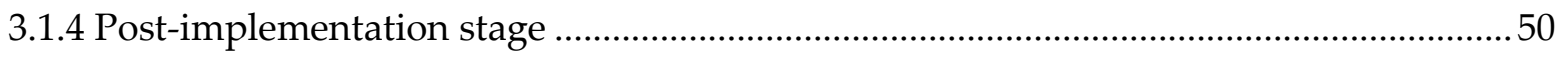

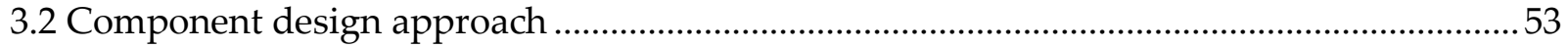

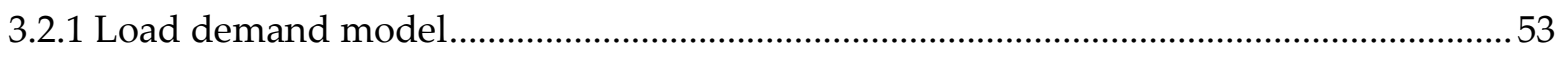

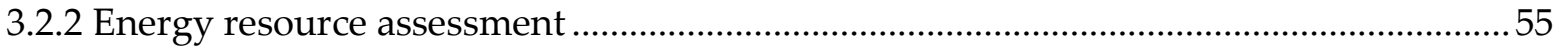

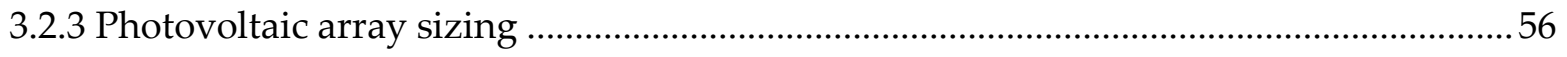

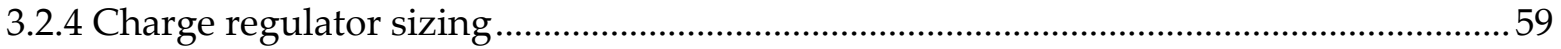

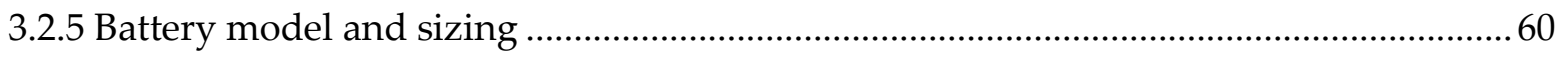

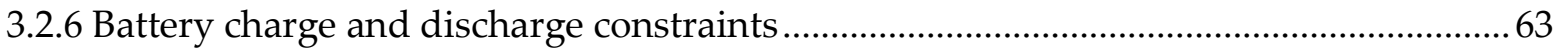

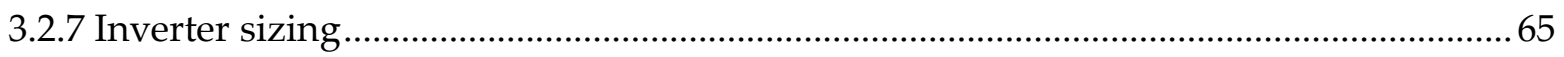

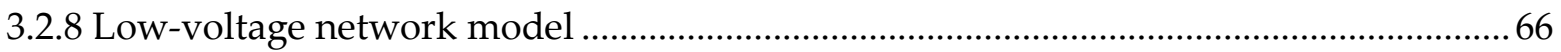

3.2.9 Diesel generator fuel consumption model .................................................................... 67

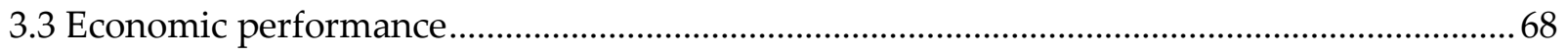


3.4 Environmental performance............................................................................................

3.4.1 Global warming potential ..............................................................................................

3.4.2 Cumulative energy demand ............................................................................................

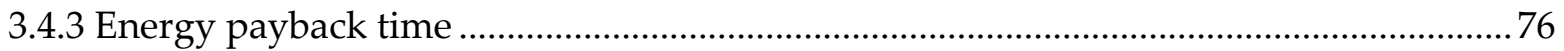

3.4.4 Energy return on investment........................................................................................

3.5 Chapter summary ………………………………………………………………………....

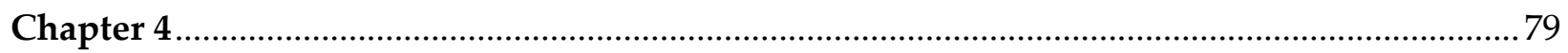

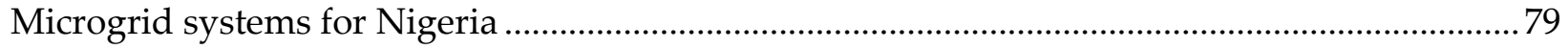

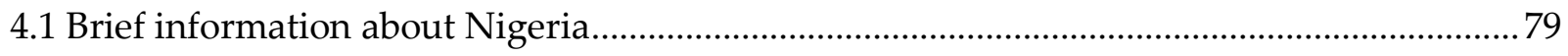

4.2 Energy situation of the country......................................................................................... 82

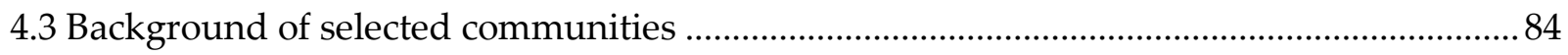

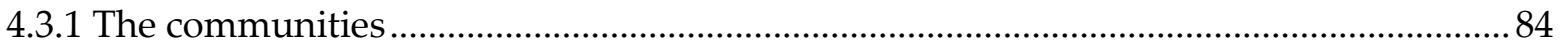

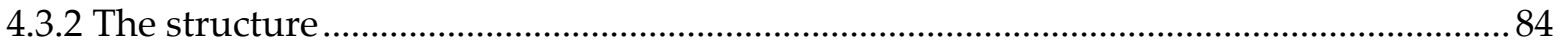

4.3.3 Assumptions made ………………………………………………………………….... 86

4.3.4 Challenges ................................................................................................................ 87

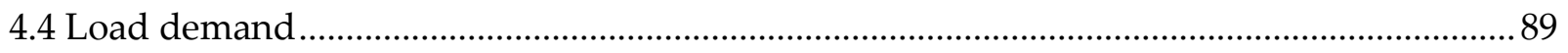

4.4.1 Users' load profile ……………………………………………………………………... 89

4.5 Solar energy resources of the locations ............................................................................... 95

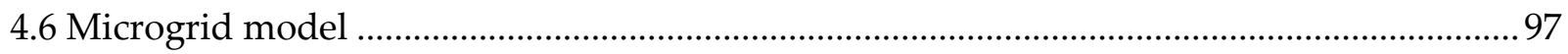

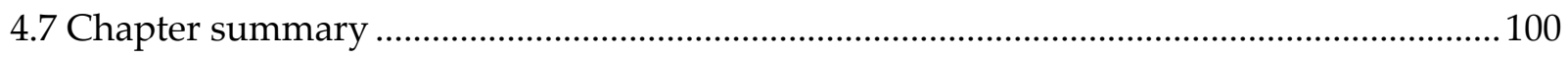

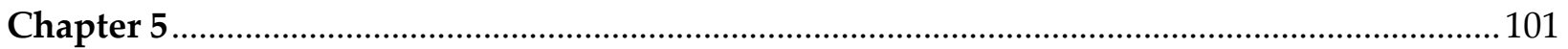

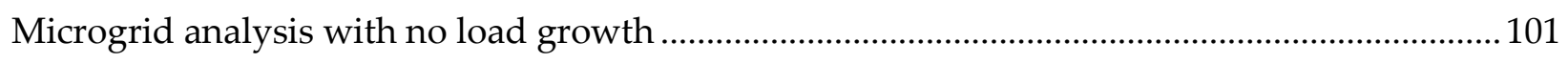

5.1 System capacity and electrical performance ……………………………………………...... 101

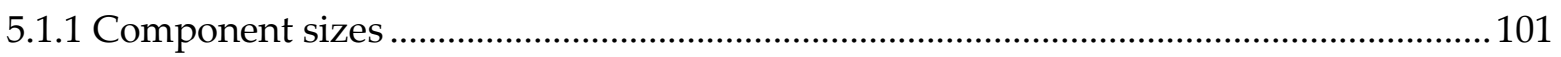

5.1.2 Daily power and energy outputs ................................................................................. 108

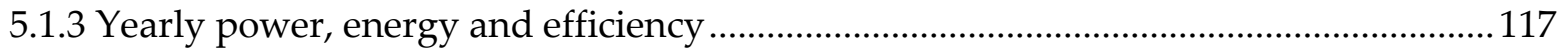

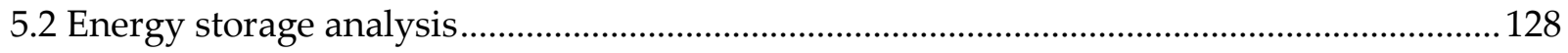

5.3 Hourly variations and reliability assessment.................................................................... 144

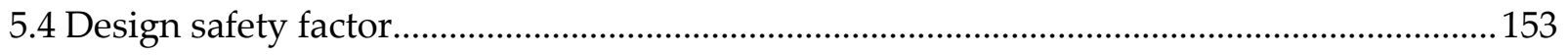


5.5 Chapter summary

\section{Chapter 6 .}

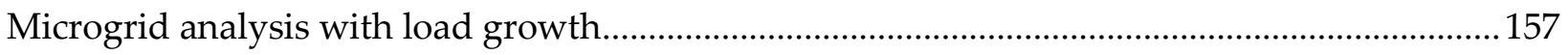

6.1 Initial system capacity and performance ........................................................................... 157

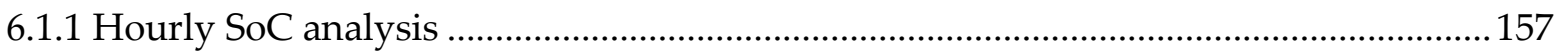

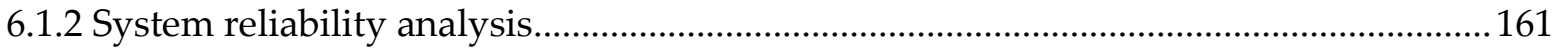

6.2 New SPM capacity with initial battery bank ................................................................... 163

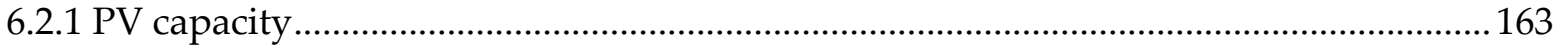

6.2.2 Battery SoC and reliability analyses with new PV capacity ….................................... 164

6.3 SPM capacity with new battery bank ................................................................................ 170

6.3.1 New PV and battery bank capacities.......................................................................... 171

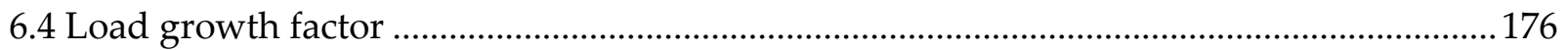

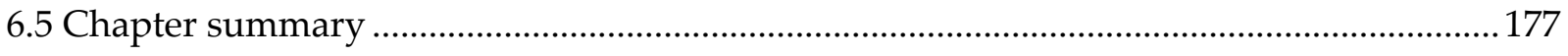

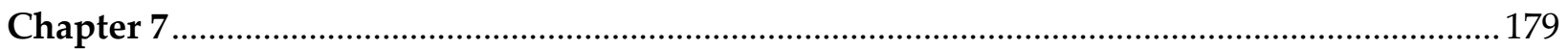

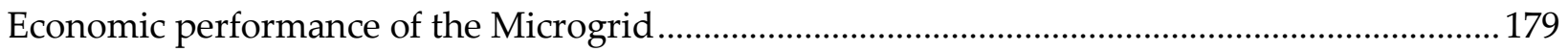

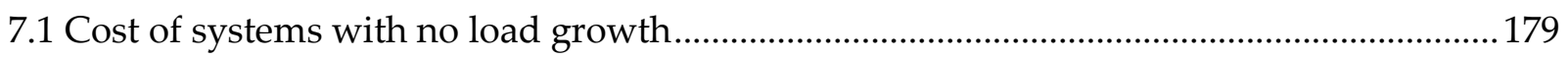

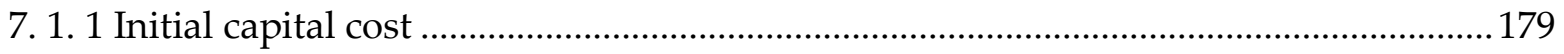

7. 1. 2 Percentage costs of PV and battery components ….................................................. 180

7. 1. 3 Operation and maintenance and replacement costs .................................................. 181

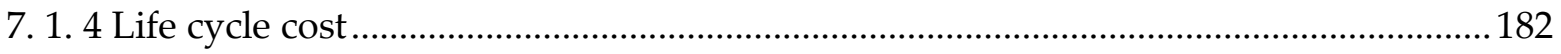

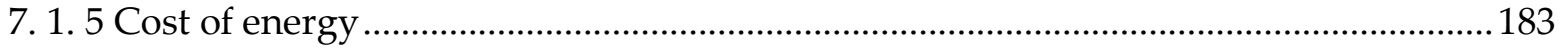

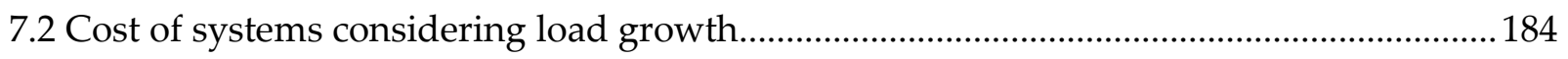

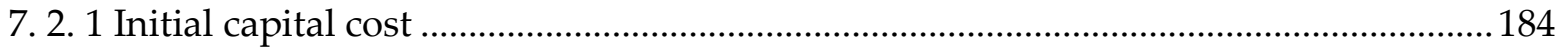

7. 2. 2 Percentage costs of PV and battery components ..................................................... 185

7. 2. 3 Operation and maintenance and replacement costs .................................................. 187

7. 2. 4 Life cycle cost ...................................................................................................... 187

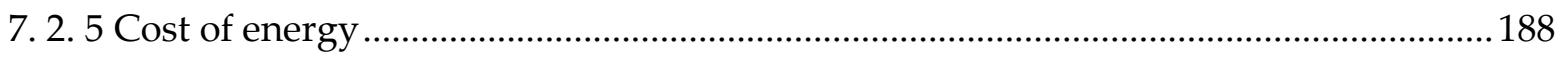

7.3 Comparison of SPM costs with a diesel plant cost........................................................... 190

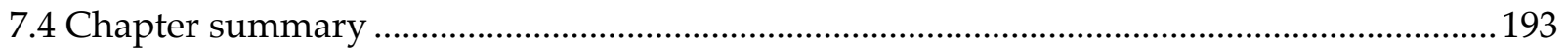


Environmental performance of the Microgrid

8.1 Carbon dioxide emissions avoided

8.2 Emission rate and GWP of the proposed SPMs.

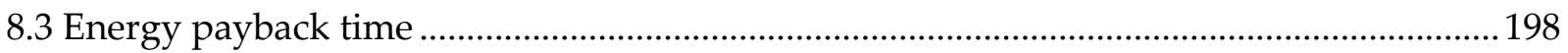

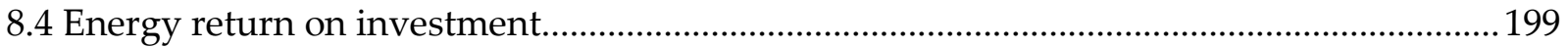

8.5 Comparison of impact of SPM systems with a diesel system...........................................201

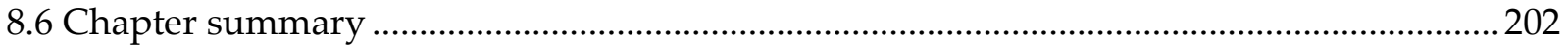

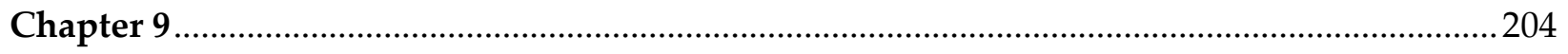

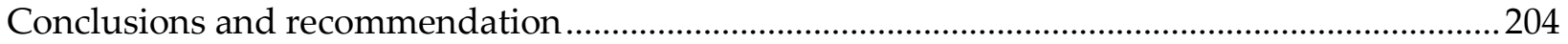



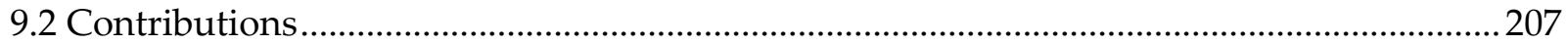

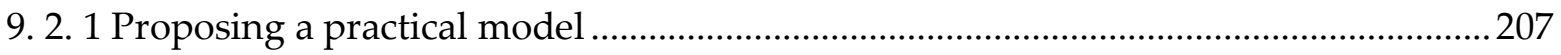

9. 2. 2 SPM capacities through design safety factor.............................................................. 208

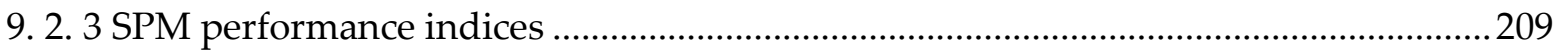

9. 2. 4 Economic performance of the proposed systems ...................................................... 211

9. 2. 5 Life cycle environmental performance of the proposed systems..............................211

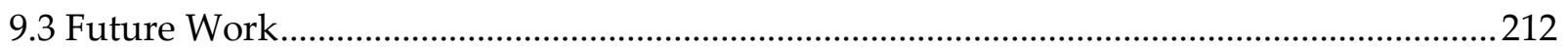

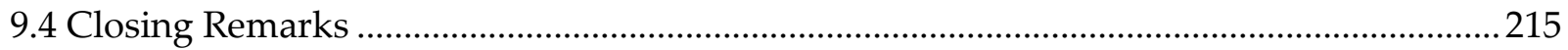

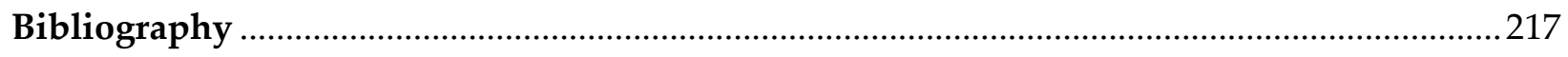




\section{List of Acronyms and Symbols}

$A_{k}$

Az

a-Si

$B_{c}(\min )$

$B_{c}(\max )$

$B_{c p}$ (req.capacity)

BSS

BOS

BoEQ

CdTe

CdS

CIS

CIGS

$C D A$

$C R_{\text {size }}$

C

c-Si

DMap

DoD

$D_{a}$

$D_{l f}$

$d_{r f}$

$d_{s f}$

$d_{j z}$

EWT

$E_{u}$

Ediff

EGEN

$E_{a G E N}$

FPVMPA

FF

GaAs

$\mathrm{G} a$

$G_{s t c}$

GHG

I number of energy users within the user category

category of users

amorphous silicon

minimum allowable battery capacity $(\mathrm{kWh})$

maximum allowable battery capacity (kWh)

battery capacity based on the desired MDOD (kWh)

battery storage system

balance of system

bill of engineering quantity

cadmium telluride

cadmium sulphide

copper indium selenide

copper indium gallium selenide

community development association

size of charge regulator @ system voltage

battery capacity ratio

crystalline silicon technology

data map

depth of discharge (\%)

days of autonomy

daily load factor

derating factor

design safety factor

duration of operation of load within a category of users

emitter wrap through

total energy demand (kWh/day)

hourly energy difference (kWh)

hourly energy produced by the PV plant (kWh)

module's yearly energy production (kWh)

fundamental PV module performance analysis

fill factor

gallium arsenide

solar irradiance of the location $\left(\mathrm{W} / \mathrm{m}^{2}\right)$

solar irradiance at STC (i.e. $1000 \mathrm{~W} / \mathrm{m}^{2}$ )

greenhouse gas

output current of solar cell (A) 


\begin{tabular}{|c|c|}
\hline IEEE & institute of electrical and electronic engineers \\
\hline IEC/TS & international electrotechnical commission/technical standard \\
\hline$I n v_{\text {size }}$ & inverter size $(\mathrm{kVA})$ \\
\hline$i$ & hourly interval \\
\hline$I_{l}$ & photo or solar generated current (A) \\
\hline$I_{d}$ & diode current $(\mathrm{A})$ \\
\hline$I_{s h}$ & shunt- leakage current (A) \\
\hline$I_{m p p}$ & maximum power current $(\mathrm{A})$ \\
\hline$I_{s c}$ & short circuit current $(\mathrm{A})$ \\
\hline$j$ & the kind of electrical appliances \\
\hline$k$ & battery rate constant $(/ \mathrm{hr})$ \\
\hline$K_{v}$ & temperature coefficient of voltage $\left(\% /{ }^{\circ} \mathrm{C}\right)$ \\
\hline$K_{p}$ & temperature coefficient of power $\left(\% /{ }^{\circ} \mathrm{C}\right)$ \\
\hline KiBaM & kinetic battery model \\
\hline Lav & average electrical load $(\mathrm{kW})$ \\
\hline$l_{g f}$ & Load growth factor \\
\hline$L p k$ & peak electrical load $(\mathrm{kW})$ \\
\hline$L_{i}$ & electrical load at time $t_{i}(\mathrm{~kW})$ \\
\hline$L_{n}$ & electrical load at time $t_{n}(\mathrm{~kW})$ \\
\hline LCCA & life cycle cost analysis \\
\hline LDTTE & losses due to temperature effect \\
\hline$L O E$ & loss of energy \\
\hline LOEP & loss of energy probability \\
\hline Lind & inductive loads $(\mathrm{kW})$ \\
\hline Lo & other loads $(\mathrm{kW})$ \\
\hline Mono c-Si & monocrystalline \\
\hline$M D o D$ & maximum depth of discharge (\%) \\
\hline MPP & maximum power point \\
\hline MPPT & maximum power point tracker \\
\hline$m$ & number of segments or intervals \\
\hline NOCT & nominal operating cell temperature \\
\hline$N_{\text {years }}$ & expected battery life span (years) \\
\hline$N_{\text {cycles }}$ & battery cycle life \\
\hline$n$ & number of hours in a day \\
\hline$n_{\text {id }}$ & ideality factor \\
\hline$n_{j z}$ & number of loads within category of users \\
\hline$n_{8}$ & conversion coefficient from primary to electrical energy \\
\hline $\mathrm{PSH}$ & minimum monthly peak sun hour \\
\hline$P W M$ & pulse-width modulated inverter \\
\hline
\end{tabular}




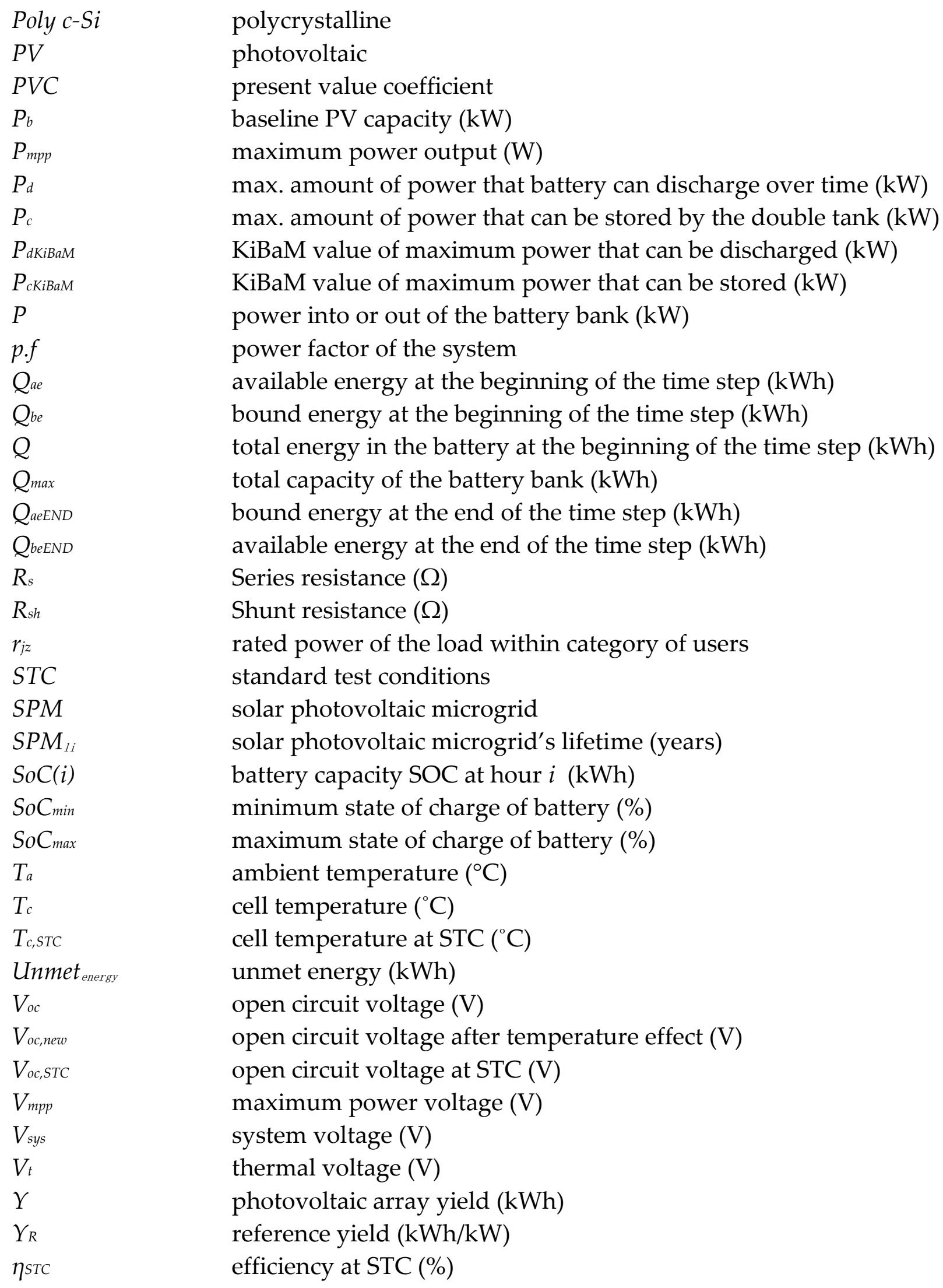




$\begin{array}{ll}\eta_{\text {new }} & \text { Actual efficiency }(\%) \\ x & \text { number of PV modules } \\ z & \text { category of users } \\ \Delta t & \text { length of the time step }(\mathrm{h}) \\ \alpha_{c} & \text { maximum charge rate of the battery (A/Ah) } \\ \eta_{c} & \text { battery charge efficiency } \\ \eta_{d} & \text { battery discharge efficiency } \\ \eta_{r t p} & \text { round trip efficiency }(\%)\end{array}$




\section{List of Figures}

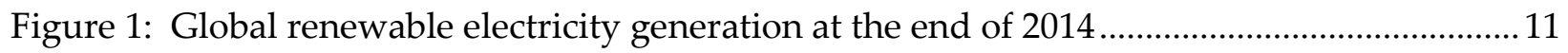

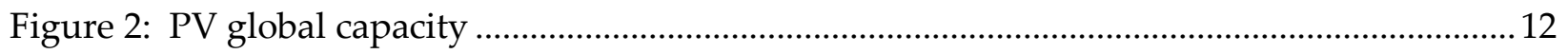

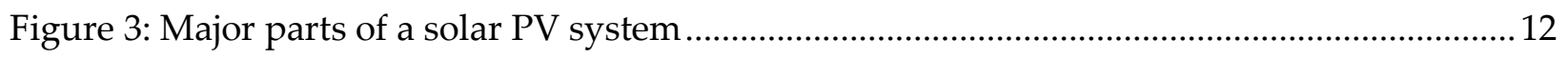

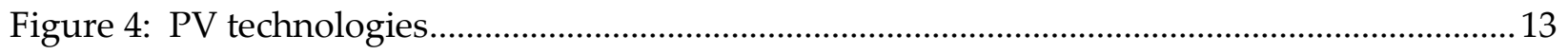

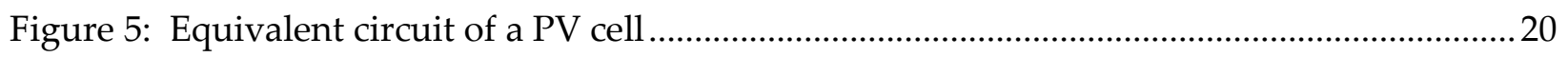

Figure 6: Module current and voltage at different irradiance levels ............................................22

Figure 7: Module current and voltage at different temperature values .........................................2 22

Figure 8: Classification of energy storage technologies..................................................................25

Figure 9: Stages of energy project ............................................................................................ 47

Figure 10: Solar energy resources across the geo-political zones of Nigeria [150] ....................... 82

Figure 11(a) - (h): Load profiles of the households..................................................................93

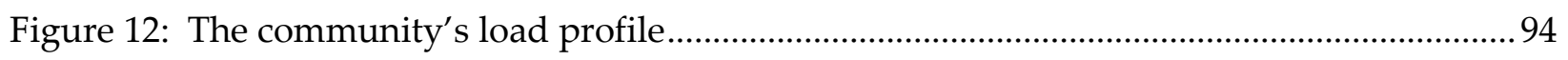

Figure 13: Peak sun hours of the northern zones .......................................................................96

Figure 14: Peak sun hours of the southern zones .........................................................................96

Figure 15: Ambient temperature of the northern zones ..............................................................97

Figure 16: Ambient temperature of the southern zones...............................................................97

Figure 17(a) Schematic of the community (b) SPM model..............................................................99

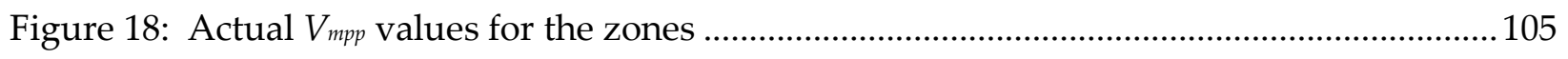

Figure 19: Corresponding ambient temperatures for the zones.................................................. 105

Figure 20: Corresponding PV module temperatures for the zones ........................................... 106

Figure 21: 190W module power output after temperature losses ............................................... 106

Figure 22: $190 \mathrm{~W}$ module power output after all losses are considered....................................... 107

Figure 23: Efficiency of the PV module for the zones ................................................................ 108

Figure 24: PV array power versus load demand for NE zone .................................................... 109

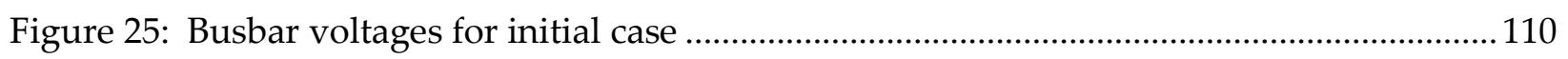

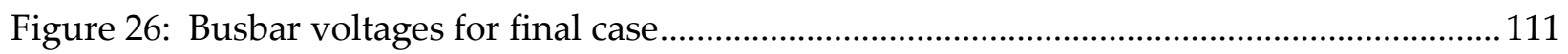

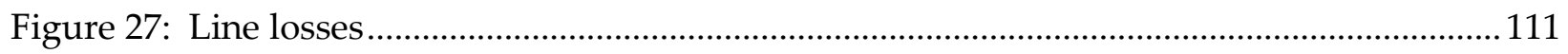

Figure 28: PV array power versus load for the NW zone ......................................................... 112

Figure 29: PV array power versus load for the NC zone...........................................................113

Figure 30: PV array power versus load for the SE zone ............................................................. 114

Figure 31: PV array power versus load for the SS zone............................................................115

Figure 32: PV array power and the load for the SW zone....................................................... 116

Figure 33: SPM systems' baseline power outputs ..................................................................... 122

Figure 34: SPM systems' power outputs after temperature losses ............................................ 123 
Figure 35: SPM systems' power outputs after temperature and other losses 123

Figure 36: SPM systems' total power losses ................................................................................ 124

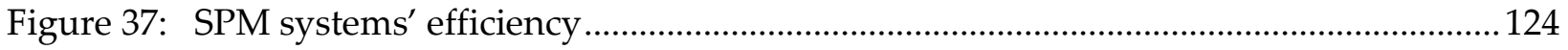

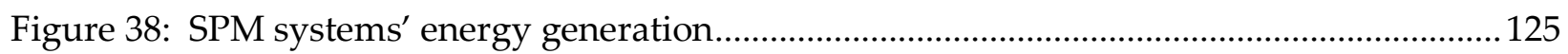

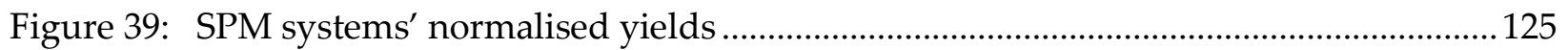

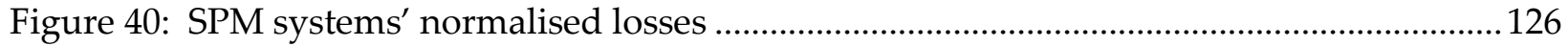

Figure 41: Battery SoC values for the initial scenario ................................................................ 131

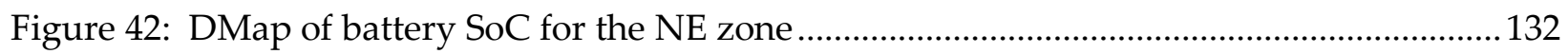

Figure 43: DMap of battery SoC for the NW zone …................................................................ 132

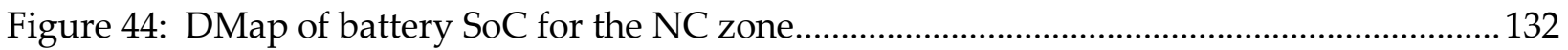

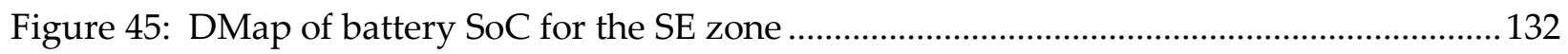

Figure 46: DMap of battery SoC for the SS zone ........................................................................ 133

Figure 47: DMap of battery SoC for the SW zone..................................................................... 133

Figure 48 (a) - (f): Frequency distribution of the battery SoC ...................................................... 134

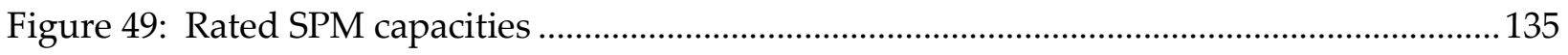

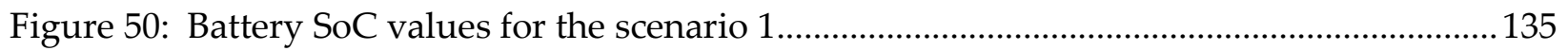

Figure 51: Scenario 1 battery SoC DMap for the NE zone ........................................................... 135

Figure 52: Scenario 1 battery SoC DMap for the NW zone......................................................... 136

Figure 53: Scenario 1 battery SoC DMap for the NC zone ........................................................... 136

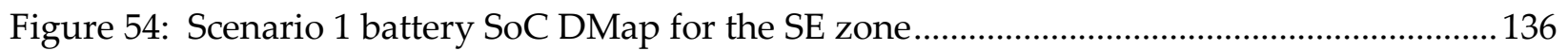

Figure 55: Scenario 1 battery SoC DMap for the SS zone ............................................................ 136

Figure 56: Scenario 1 battery SoC DMap for the SW zone .......................................................... 136

Figure 57 (a) - (f): Frequency distribution of SoC ..................................................................137

Figure 58: Battery SoC for 5\% load demand reduction ................................................................ 140

Figure 59: Battery SoC for 10\% load demand reduction .............................................................. 140

Figure 60: Battery SoC for 15\% load demand reduction ............................................................ 141

Figure 61: Battery SoC for 20\% load demand reduction .............................................................. 141

Figure 62: Battery SoC for 25\% load demand reduction ............................................................ 141

Figure 63: Scenario 2 battery SoC DMap for NE zone ................................................................. 142

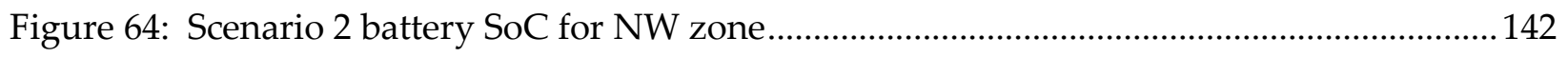

Figure 65: Scenario 2 battery SoC DMap for NC zone............................................................... 142

Figure 66: Scenario 2 battery SoC DMap for SE zone .................................................................. 142

Figure 67: Scenario 2 battery SoC DMap for SS zone............................................................... 142

Figure 68: Scenario 2 battery SoC DMap for SW zone................................................................. 143

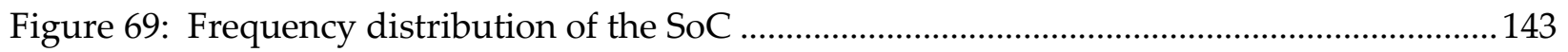

Figure 70: Hourly SoC values for the NE zone ............................................................................. 146

Figure 71: Hourly SoC values for the NW zone ......................................................................... 146 
Figure 72: Hourly SoC values for the NC zone 147

Figure 73: Hourly SoC values for the SE zone ........................................................................... 147

Figure 74: Hourly SoC values for the SS zone .......................................................................... 148

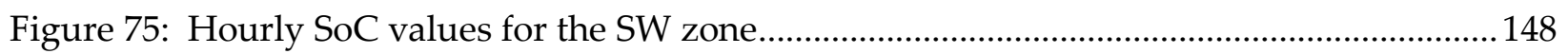

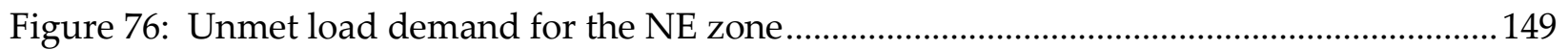

Figure 77: Unmet load demand for the NW zone .................................................................... 149

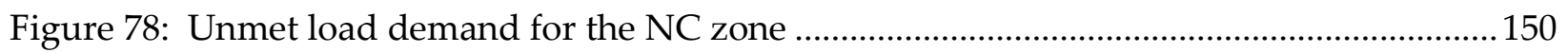

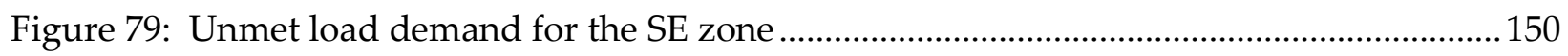

Figure 80: Unmet load demand for the SS zone ........................................................................ 151

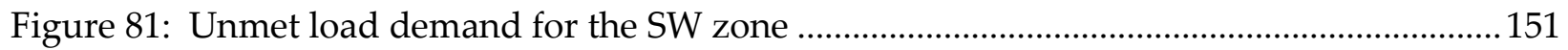

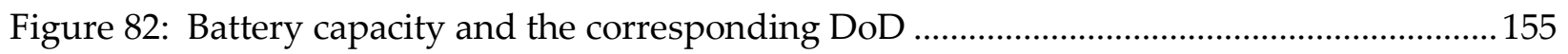

Figure 83: Battery SoC for load growth for the NE zone............................................................. 159

Figure 84: Battery SoC for load growth for the NW zone ......................................................... 159

Figure 85: Battery SoC for load growth for the NC zone ........................................................... 160

Figure 86: Battery SoC for load growth for the SE zone............................................................ 160

Figure 87: Battery SoC for load growth for the SS zone ........................................................... 161

Figure 88: Battery SoC for load growth for the SW zone ............................................................ 161

Figure 89: Unmet energy demand for the load growth ............................................................... 162

Figure 90: Loss of energy probability for the load growth........................................................... 163

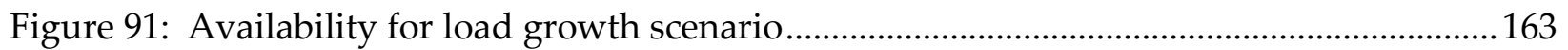

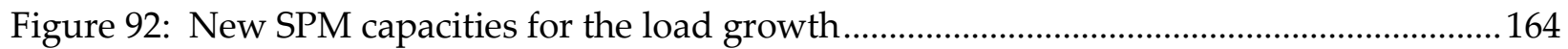

Figure 93: Battery SoC for 12.5\% load growth with new SPM capacities ................................... 166

Figure 94: Battery SoC for 25\% load growth with new SPM capacities ...................................... 167

Figure 95: Battery SoC for 37.5\% load growth with new SPM capacities ................................... 167

Figure 96: Battery SoC for 50\% load growth with new SPM capacities ....................................... 168

Figure 97: Unmet demand with the initial and the new SPM capacities ................................... 168

Figure 98: Loss of energy probability with the initial and the new SPM capacities....................169

Figure 99: Availability for the initial and the new SPM capacities ............................................ 169

Figure 100: Energy production and total losses for the new SPM capacities .............................. 170

Figure 101: Yield and losses of the new SPM capacities...........................................................170

Figure 102: Battery SoC for 150\% UD with new PV and battery capacities ............................... 173

Figure 103: Battery SoC for 175\% UD with new PV and battery capacities ................................173

Figure 104: Unmet energy demand for the new PV and battery capacities ............................... 174

Figure 105: Loss of energy probability for new PV and battery capacities................................. 174

Figure 106: Availability for the new PV and battery capacities ................................................ 175

Figure 107 (a) - (e): Load capacities for the 100, 112.5, 125, 137.5 and 150\% UDs .....................176

Figure 108: Initial capital costs of the microgrid systems with no load growth ......................... 180 
Figure 109: Percentage cost of the PV array and the battery bank ........................................... 181

Figure 110: Operation and maintenance costs of the microgrids ................................................. 182

Figure 111: Life cycle costs of the photovoltaic microgrids ........................................................ 183

Figure 112: Cost of energy of the photovoltaic microgrids.......................................................... 184

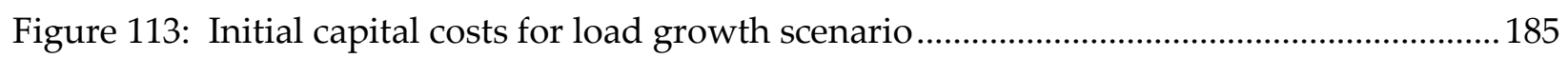

Figure 114: Percentage cost of the PV array with load growth ...................................................186

Figure 115: Percentage cost of the battery bank with load growth............................................... 186

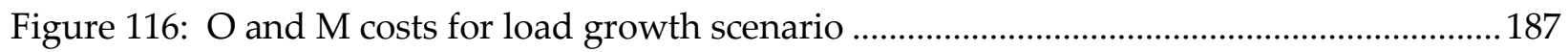

Figure 117: Life cycle costs for the load growth scenario ............................................................ 188

Figure 118: Cost of energy for the load growth scenario …..........................................................188

Figure 119: Daily fuel consumption for different demand requirements ................................... 192

Figure 120: Fuel and life cycle costs of the diesel generator ...................................................... 192

Figure 121: Cost per unit energy generation of the diesel power plant ......................................193

Figure 122: Annual carbon dioxide emission by the diesel power plant .....................................196

Figure 123: The proposed SPM's GWP …................................................................................... 198

Figure 124: The energy payback time of the SPM ...................................................................... 199

Figure 125: The energy return on investment of the SPMs ...........................................................201 


\section{List of Tables}

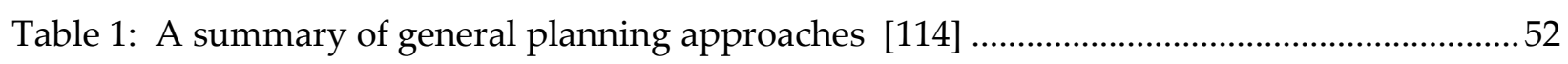

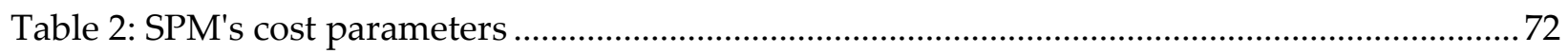

Table 3: Diesel power system's cost parameters ..........................................................................73

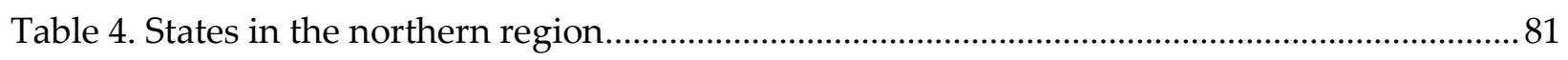

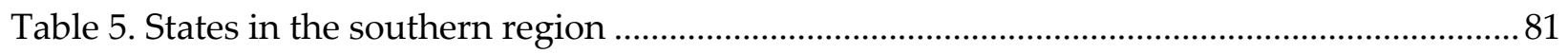

Table 6: List of selected communities............................................................................................ 84

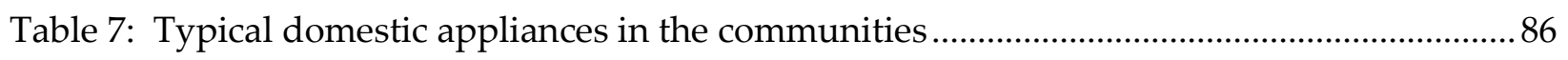

Table 8: Loads and energy consumption of the houses...................................................................94

Table 9: Arrangement of loads in the LV network .......................................................................99

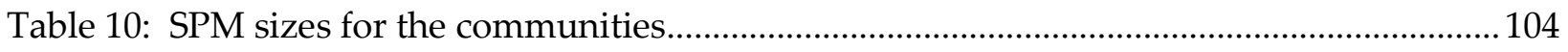

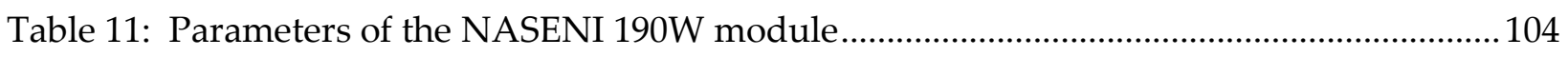

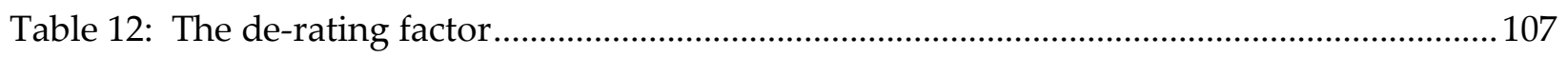

Table 13: Performance indices of the SPM systems...................................................................128

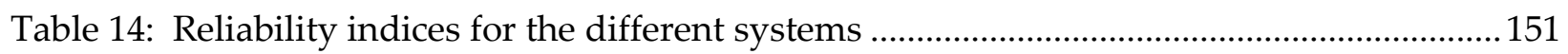

Table 15: Design safety factor for the zones ............................................................................. 154

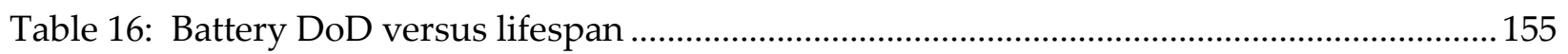

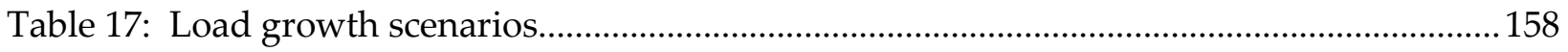




\section{Chapter 1}

\section{Introduction}

This chapter introduces the thesis and includes a brief background and scope, the motivations, the research goals, the possible contributions and their usage, and the organisation of the thesis.

\subsection{Brief background and scope}

It is reported that about 1.285 billion people in the world today, do not have access to modern electricity services (i.e. $~ 15 \%$ of the global population) [1, 3]. Of those, around 621 million people live in the sub-Saharan African region. The region's electrification rate is limited to $32 \%$, of which the urban electrification rate is $59 \%$, while the rural access rate is only $16 \%$. The lack of access to modern electricity is one of the major factors responsible for the social and economic incapacitation of most of the people in this region [4-6]. This describes the hampered socioeconomic development experienced in a country like Nigeria.

Despite Nigeria's huge population of about 182 million, about $60 \%$ of its people has no access to electricity. Even in many of the communities that have access to the national grid, electricity supply has been characterised as erratic and poor. The centralised electricity apparatus is weak and inefficient, whose outputs are not able to support the electric load demands of all the users [4, 7]. It is clear that there is little or no difference between the grid-connected and off-grid areas, because of the poor state of the existing grid systems [7]. This situation has led to a geometric increase in the rate of use of diesel/petrol generating systems for satisfying electricity demands in different parts of the region [4-8]. This has environmental and health implications, as well as high operational and maintenance costs.

Although it is usually believed that developing countries produce relatively lower carbon footprints because of their low level of industrialisation, the global warming and climate change issues affect the entire world. Therefore, there is a paramount need to improve the situation through deployments and application of clean energy technologies [5]. Such energy technologies will be needed as an alternative to 
the carbon-intensive technologies that are currently depended on in several communities, for meeting the users' energy demands.

In a bid to respond to the poor electricity situation, some of the governments, project developers, investors, the private sector, NGOs and policymakers within the African region, including in Nigeria, have begun to take the issue of energy very seriously. It has become one of the priority items on their agenda for growth and development. This is opening new opportunities for the deployments and application of remote microgrid technologies based on renewable energies. Such electricity systems have the capability to meet the users' daily energy needs in the isolated areas.

The main focus of this research is developing new electrical systems for remote residential dwellings in different regions of Nigeria that currently do not have access to the national grid. It intends to explore the available energy resources of those locations to meet the intended users' basic energy needs.

Electrical power systems are basically categorised into system planning and system operation and management. While system planning concentrates on the pre-design analysis and the detailed engineering design of energy systems, the system operation and management focus on the energy project implementation and post-implementation issues. The scope of this thesis is therefore limited to, system planning, which typically considers both pre-design and detailed design aspect of the energy development for offgrid communities.

\subsection{Motivation}

Solar photovoltaic microgrid systems are progressively gaining recognition and relevance in the electricity industries, both in the developing and developed nations. In developed countries, such systems are increasingly being integrated with the existing centralised electricity infrastructure, primarily to ensure energy security and reduce the use of carbon-intensive energy systems. On the other hand, the systems could play a crucial role in electrifying remote communities of the developing countries, in the shortor medium- term $[6,7]$.

The access to electricity and its contribution to the economic growth and the elimination of poverty are part of the UN's programmes. The UN declared the eight Millennium Development Goals (MDGs) in the year 2000, for the purpose of getting rid 
of extreme poverty and hunger, reduce maternal and child mortality, tackling diseases HIV/AIDS, malaria etc. It also advocated gender equality and the empowerment of women. It was hoped that achieving these goals would ensure the sustainability of the global environment and foster worldwide cooperation for development [9].

The MDGs have been the most widely supported worldwide development goals ever in the history of humanity. They have also created the necessary awareness about the compelling need for the well-being of the people. They brought to the fore a wide range of programmes and projects on clean energy technologies, as a means of mitigating carbon emissions. They also give some directions for research in the area of renewable energy around the world.

Also, UN's recent Sustainable Development Goals (SDGs) are intended to ensure that the whole world is electrified by 2030. The electricity needs of the 1.285 billion people around the globe without access to modern electricity services are strongly emphasised [7, 10-17]. One of SDGs objectives is to provide access to affordable, reliable, sustainable and modern energy for all. Undoubtedly, the availability of energy services will be instrumental to realising socio-economic growth and sustainable development in any nation. Furthermore, the UN recently declared the years 2014 to 2024 as the decade of "Sustainable Energy for All" (SE4All). This agendum stresses electricity access, doubling the worldwide improvement in energy efficiency practices and increasing renewable energy production in the global energy capacity by $100 \%$ [10].

Plans are also in place in the African region towards realising an increased electricity access rate for its people. One such effort has been creating the African Energy Leaders Group (AELG). This was intended to place energy issues as a priority on the African continent's economic agenda. This would then foster the creation of facilities and infrastructure that offer increased access to reliable and cost-efficient energy services. It also includes the widespread application of energy efficient appliances by the year 2030 [10]. The two regional sub-groups supporting the AELG initiative are currently established in West Africa (including Nigeria) and East Africa.

The ease of development and the perceived environmental friendliness have been identified as one of the factors that make off-grid renewable energy systems promising electricity solutions for isolated communities. This particular aspect of research is currently receiving a growing attention, and it is expected to thrive in the near future. This is because off-grid solutions based on renewable energy resources have the potential to offer a cost-effective electricity supply for those communities, compared to 
the conventional diesel power generation option [7]. The total dependence on the expansion of the existing grid infrastructure alone cannot achieve the desired electricity access increase in the short-term. This is another key factor that favours the off-grid electricity systems $[6,16]$.

This thesis is motivated by the energy situation of the people living in the offgrid communities. Compared to some existing related research works in the literature, its particular focus lies in proposing more practical energy solutions. Access to energy in remote communities may be acquired in three different ways [16]:

1) At the household level by employing isolated devices that are dedicated to generating energy

2) At the community level where there is a single energy infrastructure that caters for the whole community

3) At the grid-based level where the existing national grid infrastructure is extended to the community

This thesis focuses on the second option, i.e. energy system at the community level. Different remote communities in Nigeria have been selected for this study, whose solar energy resources could be harnessed to address the prevailing energy challenges. Community-based renewable energy systems are new in the country; this gives the thesis an opportunity to reach some useful conclusions. These can be useful for understanding the important indices necessary for planning and developing energy systems for isolated communities.

\subsection{Thesis Goal}

The overall aim of this thesis is to propose Solar Photovoltaic Microgrids (SPMs) for residential applications. Different remote communities are used as case studies, within Nigeria' six geo-political zones. The main focus of the research is to create new electrification systems for those communities, carefully considering their basic energy needs and the available solar energy resources. Such energy systems could be practically deployed to address the energy challenges encountered in those communities.

Certain questions are raised when renewable energy technologies are being considered for electricity generation, such as the following: 
- How to determine the appropriate electricity generation capacity that is able to adequately support the basic energy requirements of the houses within a specified community?

- How a solar photovoltaic microgrid system (SPM) can be depended on for meeting the users' load demand for every hour of the day?

- How reliable will the microgrid system be with and without load growth?

- To what degree is the solar photovoltaic energy system economically and environmentally viable?

These inquiries indicate that different people are involved in this process, with different loads and energy consumption patterns. There is also the possibility of changes in the daily and seasonal electricity demands by different people or households. The intermittent characteristic of renewable energy sources makes solar electricity a relatively difficult option for supplying continuous electricity to the intended communities. The eventual expansion of the communities that can lead to a load growth in the future is also identified as a potential challenge.

\subsubsection{Research Objectives}

This research intends to look at the above-mentioned issues closely so as to arrive at useful contributions that can advance energy research for isolated areas. Therefore, in order to address the mentioned challenges, with the prime goal of the study in focus, the following research objectives have been established:

1) To assess the intended users' daily energy needs and usage patterns in the specified communities and create an aggregate load profile for 24 households per community

2) To design and model stand-alone SPMs with electrical generation capacities that can balance the users' energy usage

3) To evaluate the SPM's performance during different seasons - dry and rainy seasons and then assess its availability over the year, with and without load growth

4) To conduct comparative cost and environmental benefit evaluations for the SPMs and the diesel plant option 
The first objective seeks to get a sound understanding of the users' basic energy demands. This is then applied to develop the electric energy consumption profile. This considers the users' needs and preferences. It is expected that their energy usage will vary due to the daily and the seasonal variations. For instance, while electric fans become necessary during the dry season, they are less important during the rainy season because of the change in seasons. The communities' aggregate electric load profile will provide useful information about the amount of electricity that needs to be produced to meet the people's daily energy requirements.

The second objective relies on the first objective to create suitably-sized solar electricity systems for the communities, in relation to the specified locations' solar energy resources. The locations' resources will also be assessed, so as to be able to predict the amount of electricity that may be delivered over the year. Different energy configurations will be modelled, whose techno-economic design and analysis will then be compared with that of a diesel power system.

The third objective concentrates on how to quantify the system's performance in light of the locations' environmental factors - solar irradiation and ambient temperature. It also assesses the proposed SPM's reliability over the year. Thus, the periods within the year when there could be a loss of energy supply can be identified. The focus of the fourth objective is to analyse the proposed system's economic and environmental performances and then compare them with that of a diesel system.

With these objectives, the thesis is poised to examine the task of proposing or developing community-based energy systems from a practical point of view. It will also assess the users' daily energy patterns and requirements, in relation to the site characteristics. This will ensure the design, modelling and simulation of appropriate energy generation systems. The solar electricity system's performance will be evaluated through a quantitative technique. The outputs of the analysis will then by compared with $100 \%$ diesel energy system and with some existing related works. The detail of this will be discussed in the subsequent chapters.

\subsection{Possible Thesis Contributions and their Usage}

This thesis makes the following contributions to the body of knowledge in the field of renewable energy systems: 
1) The proposed SPMs can provide a reference point for researchers, designers, planners, policymakers, and the stakeholders of interest in designing, planning and developing new solar electrification systems for remote communities. The research is also expected to provide important information about the key energy design and planning indices, such as the users' energy needs and the electrical load patterns, the location's solar energy resource, the possibility of load demand growth in the future and the appropriate power generation capacity

2) The research will also reveal the impact of the solar insolation and the ambient temperatures on the proposed SPM's rated generation capacities for different locations. Such an analysis will consider the worst-case users' load demand requirements. A detailed technical analysis relating the users' demands with the SPMs generation will provide an insight into the possibility of providing a 24hour energy supply for the intended communities. The design safety factor that is introduced in the design approach could be used as a yardstick for determining suitable system's capacities for PV energy application in the locations. The thesis, therefore, can make a contribution by ensuring a good understanding of the design and better utilisation of solar energy resources

3) The thesis will also provide a better academic understanding of the solar photovoltaic system's performance. This accounts for the possible system's energy production and the losses in the different localities. The SPM's reliability indices (i.e. unmet load demand, loss of energy probability and the availability) that are assessed in this study will provide useful information about the confidence level at which the proposed energy systems can meet the users' load demands

4) The economic analysis provides general indications that the proposed SPMs have the potential to offer a cost-effective electricity supply, compared to the diesel plant. The SPM's environmental assessment demonstrates how much fuel and $\mathrm{CO}_{2}$ emissions could be saved. It will also be useful for understanding the proposed system's life cycle impact, in terms of the emission rate, global warming potential, cumulative energy demand, energy payback time and the energy return on investment

These contributions have already been published in peer-reviewed journal articles and technical conferences (see the publication section). 


\subsection{Thesis Structure}

The remaining part of the thesis is organised as follows:

1) Chapter 2 presents the background of the thesis. This discusses the general overview of renewable energy systems. It then surveys in detail the state-of-theart of solar photovoltaic and energy storage systems. It reviews existing related works and briefly describes the global engineering standards consulted for the study

2) Chapter 3 discusses microgrid planning strategy. It focuses on the concept of system planning strategy - pre-design and detailed engineering analysis, system sizing and design, modelling and simulation and component selection. It assesses and discusses the intended users' load demands and the available energy resources. It also presents the performance analysis in terms of energy production and losses, economic and environmental metrics. The approaches are based on two scenarios, i.e. microgrid with no load growth and microgrid with load growth

3) Chapter 4 presents the background information about Nigeria. It discusses the energy situation of the country, the available solar energy resources and the layout of the community and the households. It also presents the status of the intended energy users and their lifestyles

4) Chapter 5 presents a microgrid system analysis with no load growth. It discusses the engineering design and analysis, focusing on system capacities and detailed electrical performance, in relation to users' load demands. It also focuses on the energy storage and availability evaluations. It presents the results for different battery state of charge scenarios and the reliability of different sizes of microgrids

5) Chapter 6 presents a microgrid system analysis with load growth. It discusses the engineering design analysis for the corresponding system capacities and their electrical performances. It presents the results for different battery state of charge scenarios and the reliability of different sizes of microgrids

6) Chapter 7 focuses on the economic performance of the photovoltaic microgrids. It also compares the economic analysis of the proposed energy systems with that of a diesel power system 
7) Chapter 8 focuses on the life cycle environmental performance of the microgrid systems. It also compares the environmental impacts of the proposed energy systems with that of a diesel power generation plant

8) Chapter 9 concludes the thesis and provides directions for future work. It is divided into the achieved objectives of the thesis, the main contributions of the research, the future work and the concluding remarks 


\section{Chapter 2}

\section{Background}

This chapter provides a relevant background for the thesis and is divided into six main parts. The first part presents an overview of renewable energy systems, while the second part focuses on a detailed survey of the global developments in solar photovoltaic technologies. The third section describes the solar photovoltaic cell and module models, including some fundamental PV terms. The fourth part of the chapter presents the state-of-the-art of energy storage technologies for electrical power applications. The fifth section presents the review of related existing work, while the sixth part of the chapter provides a brief description of the global engineering standards that are relevant to the thesis. A summary concludes the chapter.

\subsection{Overview of Renewable Energy Systems}

Renewable energy resources are defined as energy sources that are obtained from the "natural" and continuous energy flows occurring in the "immediate environment" [13]. The word "natural" indicates that they do not require any human effort to bring about their flow or availability in the environment, unlike the fossil-based energy resources that need human effort and exploration to make them available for use. Energy supplies from renewable energy resources have been considered to be in harmony with sustainable development plans compared to non-renewable energies e.g. fossil- and nuclear-based fuels, that have the perceived issues of energy resource limitations and adverse environmental impacts [13].

While renewable energies are sometimes called "green" or "sustainable" energies, the non-renewable energies are referred to as "finite" or "brown" energies. The renewable energy sources include solar, wind, water, tidal, wave, biomass and geothermal, and they produce lesser negative impacts on the environment. Though they are naturally occurring energy sources, they exhibit a variable characteristic [14]. Hence, the electricity delivered by their technologies is fashioned according to the "timevarying" availability of the sources [15]. This feature is one of the major challenges faced in the utilisation of renewable energy resources for power applications. However, the variability is usually balanced by the application of energy storage systems. Several 
energy storage technologies exist for renewable energy applications, which will be later discussed in this thesis.

Renewable energy currently accounts for $22.8 \%$ of the total global electricity production, while fossil fuels and nuclear account for $77.2 \%$ [16]. The total worldwide electricity generation at the end of 2014 was 23,636 TWh (as shown in Figure 1), with hydro, wind, biopower and solar PV accounting for $16.6 \%, 3.1 \%, 1.8 \%$ and $0.9 \%$, respectively. Geothermal, concentrating solar power (CSP) and ocean electricity generation jointly account for $0.4 \%$ of the total electricity production [16].

In addition, the total global renewable power capacity in 2014 was 1,712 GW, with hydro, biopower, geothermal, solar PV, CSP and wind accounting respectively, for 1,055 GW, $93 \mathrm{GW}, 12.8 \mathrm{GW}, 177 \mathrm{GW}, 4.4 \mathrm{GW}$ and $370 \mathrm{GW}$ [16], compared to its capacity of $800 \mathrm{GW}$ in 2004 (i.e. $715 \mathrm{GW},<36 \mathrm{GW}, 8.9 \mathrm{GW}, 3.7 \mathrm{GW}, 0.4 \mathrm{GW}$ and $48 \mathrm{GW}$ ). This information indicates that renewable electric power capacity has appreciably increased from $800 \mathrm{GW}$ to 1,712 GW between 2004 and 2014. It is also expected that significant growth will be experienced in the renewable energy industry, in the years to come.

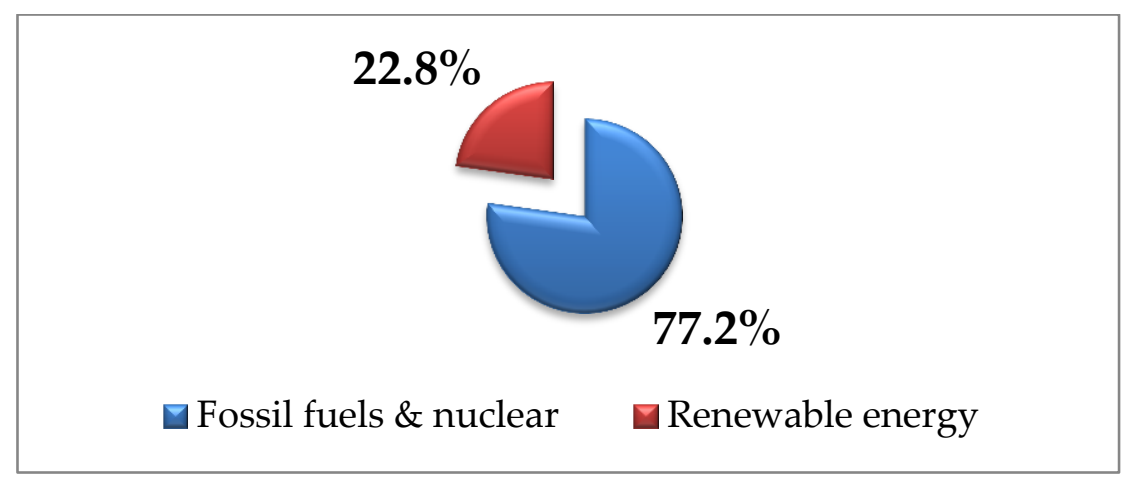

Figure 1: Global renewable electricity generation at the end of 2014

*The total global energy production in 2014 being 23,636 TWh [17]

This thesis focuses on solar energy-based systems; therefore, this section will primarily concentrate on the background of photovoltaic systems. The PV global capacity is shown in Figure 2. It is obvious that solar PV has witnessed a tremendous growth for over a decade now. It continues to become more popular and is identified as one of the promising energy options for electrifying off-grid communities around the world. 


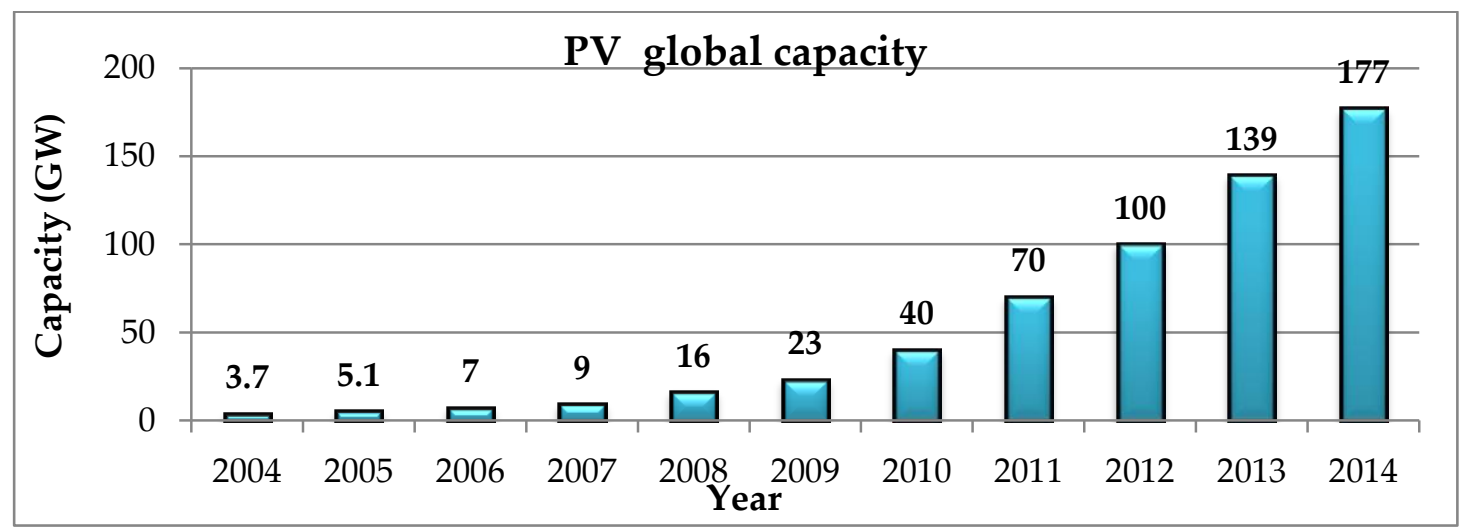

Figure 2: PV global capacity

In recent times, there has been a continuous reduction in the prices of solar PV modules [6], which is identified as one of the factors that stimulate the interests of people in solar electricity option. Apart from this, PV electricity systems are noiseless; they have no moving parts and also require relatively low maintenance. Though a part of the balance of systems (BOS) needs to be replaced during the useful life of the entire system, the PV modules have a long life span, usually over 20 years [18, 19]. The components of PV systems are classified, as shown in Figure 3 [18].

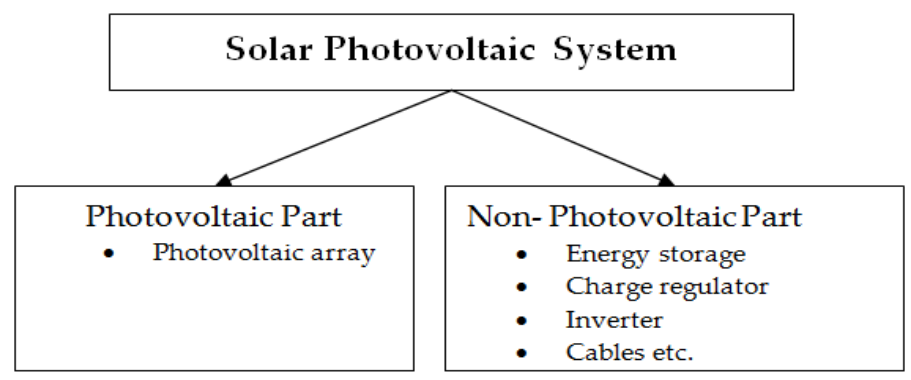

Figure 3: Major parts of a solar PV system

1) The photovoltaic part - mainly the modules

2) The non-photovoltaic part - all other components apart from the modules (i.e. the BOS). This part includes the energy storage system, charge regulator, inverter, cables, PV frame etc

\subsection{Photovoltaic technologies}

Solar photovoltaic technologies are basically categorised as shown in Figure 4 [6, 7, 20-55]. 


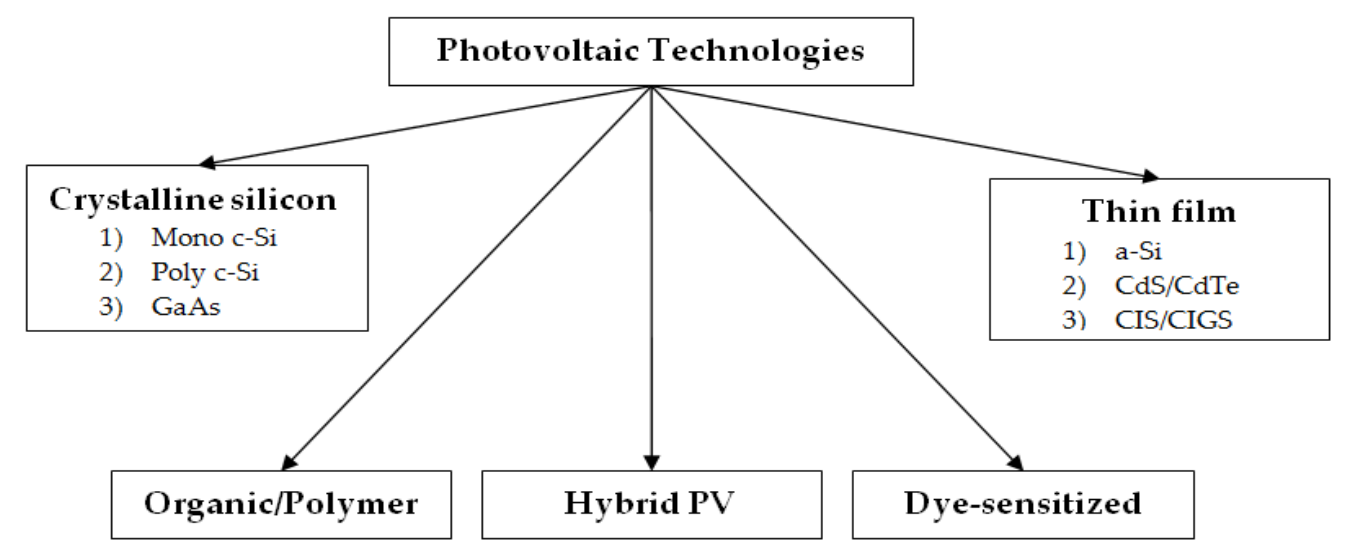

Figure 4: PV technologies

\subsubsection{Crystalline silicon (c-Si) technologies}

These systems are regarded as the "first generation of PV technologies", with silicon being the primary material for manufacturing the cells and modules. There has been a wide range of developments over time in cell/module efficiency. This is one of the factors that currently make them leaders in the solar photovoltaic market when compared to other technologies. Currently, the energy conversion efficiency of $24.7 \%$ has been reached for single crystal silicon solar cells at standard test conditions (STC) $[12,23,41,56]$. This efficiency has been obtained at a solar irradiance, temperature and air mass of $1 \mathrm{~kW} / \mathrm{m}^{2}, 25^{\circ} \mathrm{C}$ and 1.5 , respectively [18].

The costs of crystalline silicon solar cells/modules have constantly being reduced over time, especially the monocrystalline (mono c-Si) and polycrystalline (poly c-Si) cells and modules. This has also contributed to the popularity of these technologies. The c-Si technologies are classified into mono c-Si, poly c-Si, tricrystalline (tri c-Si), emitter wrap through (EWT) and the gallium arsenide (GaAs). One of the major challenges with c-Si solar PV technologies is that they are adversely affected by an increase in the location's ambient temperature, i.e. temperature $>25^{\circ} \mathrm{C}$ [18]. It is stated in the literature that the c-Si PV module temperature can be $25-35^{\circ} \mathrm{C}$ hotter than the ambient temperature [6]. This leads to a reduction in the module's output power.

- The mono c-Si is currently the most widely used solar PV, and it uses the silicon p-n junctions in its manufacturing arrangements. It is usually produced by the Czochralski (CZ) process, a technique associated with the growth of a crystal, melting of feedstock and pulling a single crystal ingot by using a seed crystal. 
The conversion efficiency of the mono c-Si cells/modules reported by manufacturers within the PV industry is between 15\% and 20\% [20-25]. Mono cSi cells are usually identified with "pseudo-square" cross sections [29-31]

- The poly c-Si is usually produced by melting the silicon material and then allowing it to solidify so as to orient the crystals in an "immovable" direction. In this way, a rectangular ingot of multi-crystalline silicon is developed, which is sliced into blocks and wafers [31]. Unlike the mono c-Si arrangements, the poly cSi cells are usually identified with "square" cross sections. This type of c-Si technology is currently a competitor to mono c-Si modules in the PV market, in terms of cost and electrical performance. Over the past few years, it is apparent that the conversion efficiency of poly c-Si cells and modules are relatively lower than those of mono c-Si types, i.e. $<15 \%$ [26, 27]. However, some poly c-Si products have evolved having the same conversion efficiency with mono c-Si modules. Such is the case of SUNTECH $250 \mathrm{~W}$ c-Si modules (both mono and poly c-Si types) [26]. Both modules have the same parameters viz. fill factor and conversion efficiency of $77.52 \%$ and $15.38 \%$, respectively. However, the maximum power output of these two modules at the nominal cell operating temperature (NOCT) are $183 \mathrm{~W}$ and $185 \mathrm{~W}$, respectively, with their corresponding temperature coefficients of power being $-0.0045\left(/{ }^{\circ} \mathrm{C}\right)$ and $-0.0043\left(/{ }^{\circ} \mathrm{C}\right)$. The implication of the disparity in the NOCT and the temperature coefficient of power is that the poly c-Si type will have a better $\mathrm{kWh} / \mathrm{kW}$ performance in tropical weather (hot weather conditions). The efficiency of poly c-Si modules is also between $15 \%$ and $20 \%$ [23, 24]

- The tri c-Si solar technology has the capability and "mechanical stability" to be sliced into thinner wafers, compared to the mono c-Si technology [19]. Such ultra-thin slicing makes it a more appropriate technology when a relatively higher mechanical yield is desired. A fill factor and conversion efficiency of $80 \%$ and $16.79 \%$ respectively, have been documented in the literature for a silicon wafer that is designed with an active cross-sectional area of $156 \mathrm{~cm}^{2}$ [22]

- The EWT technology has been developed by some solar PV manufacturers based on the concept of improved cell design. This is expected to lead to increased conversion efficiency compared to the application of the conventional material 
optimisation technique used in most other technologies [19]. Conversion efficiencies between $15 \%$ and $20 \%$ have been achieved by employing this technology. Examples of popular PV manufacturers that use EWT technology are Advent solar and SUNPOWER Corp

- The GaAs technology is made up of two major compounds, namely gallium (Ga) and arsenic (As). The literature establishes that its conversion efficiencies are higher compared to other solar photovoltaic cells/modules that are manufactured from silicon materials [27]. This is made possible by the alloy arrangements of GaAs, aluminium ( $\mathrm{Al})$, indium (In), phosphorous $(\mathrm{P})$ and antimony $(\mathrm{Sb})$ that leads to the creation of a multi-junction structure. Such a photovoltaic structure possesses a relatively high value of band gap energy (i.e. band gap of $1.43 \mathrm{eV}$ compared to $1.1 \mathrm{eV}$ for single junction arrangements). The GaAs modules have the capability to resist heat and this special property makes them suitable for concentrator photovoltaics and space applications. They are light in weight compared to the conventional mono and poly c-Si technologies. However, they are more expensive than the other technologies. The literature indicates that the highest conversion efficiency of $39 \%$ is achieved for Gallium arsenide (GaAs)/indium gallium phosphide (InGaP) multi-junction crystalline type [19, 20, 23]. Also, an efficiency of $40.8 \%$ has been reported by National Renewable Energy Laboratory (NREL) for the metamorphic triple-junction solar photovoltaic cell $[12,24]$

\subsubsection{Thin film technologies}

These technologies are thin semiconductor films. They are manufactured with the concept of material optimisation to realise relatively lower costs of manufacturing [32]. The thin film technologies include amorphous silicon (a-Si), cadmium telluride (CdTe), cadmium sulphide (CdS/CdTe), copper indium selenide (CIS) and copper indium gallium selenide (CIGS) [32-48]. They are made by depositing thin films of one of the mentioned semiconductor materials on a particular substrate. Such a substrate could be a glass or stainless steel, and the process of depositing thin layers is referred to as the sputtering process. This manufacturing method can facilitate the deposition of semiconductor materials, as thick as $<10$ micron in contrast to the c-Si technologies that have a comparatively higher wafer thickness (i.e. a typical thickness of 230 micron) [37]. 
However, due to relatively higher capture losses, the thin film technologies still have lower efficiencies than those of c-Si solar cells and modules.

- a-Si is a kind of thin film technology that is non-crystalline in nature. It is the most developed amongst the thin film technologies. Its relatively higher conversion efficiency also makes it to be very popular. Though it possesses disordered silicon arrangements, it is forty times more absorbent than the conventional mono c-Si solar cells. The disordered silicon arrangements in a-Si give it a relatively higher band gap (i.e. $1.7 \mathrm{eV}$ versus $1.1 \mathrm{eV}$ for c-Si technology). This is through the absorption of the visible part of the light spectrum than the infrared portion, but a-Si materials exhibit dangling bonds that lead to defect states in their arrangements [23]. In practice, the issue of defect states in a-Si is addressed by reacting hydrogen with it, thus, creating amorphous silicon hydride (a-Si:H). Such a material is relatively more stable than the conventional a-Si structure. The highest laboratory conversion efficiency of a single-junction aSi photovoltaic module is $12 \%$ and this is reduced to about $4 \%$ to $8 \%$ under the outdoor condition. This reduction in efficiency, when exposed to light, is referred to Staebler Wronski (SW) effect, otherwise called the light-induced degradation [32]. This is a major challenge for a-Si modules. The concept of thermal annealing and developing a multi-junction structure has been introduced to enhance the conversion efficiency and then address the issue of photo-degradation problem. Despite these efforts, the application of a-Si technology for electricity generation is hampered by its low efficiency [38]. At the moment, a-Si cells and modules are a victim of the reduction in the costs of c-Si solar cells and modules. In this case, its costs could not compete with the c-Si technologies in the market

- The CdTe technology is capable of reaching a conversion efficiency of $>15 \%$ [15], just with a small cross sectional area and a typical band gap energy of $1.45 \mathrm{eV}$, according to the literature. This feature indicates that it possesses a high direct coefficient of absorption (COA) of the small semiconductor material. Conversion efficiencies of more than $9 \%$ have been reported for CdTe solar modules, i.e. with some typical laboratory and commercial efficiencies being $18 \%$ and $11.1 \%$, respectively [39]. One of the main setbacks for this thin film technology is that cadmium is toxic, and this presents a serious environmental threat. Also, the 
availability of tellurium is another issue with this technology. These issues also limit their use for electricity production applications

- The CIGS/CIS technology currently has a developing status but is likely to compete with other thin film technologies in the future. One of its special features is that its direct band gap could be raised up to a value of $1.68 \mathrm{eV}$. The cell and module efficiencies of $20 \%$ and $13 \%$ respectively, have been mentioned in the literature [41-44]. Apart from the relatively low-efficiency values, some of the challenges with CIGS are the availability of indium and their photodegradation in heat and/or damp conditions. The photo-degradation issue requires additional barrier coatings on the thin film material

\subsubsection{Hybrid solar photovoltaics}

This kind of solar photovoltaic technology is produced from adding c-Si and non c-Si semiconductor materials. An example of the hybrid solar cell is the Sanyo product, with a conversion efficiency of $21 \%$ [40]. The Sanyo solar cell is regarded as Heterojunction with Intrinsic Thin Layers (HIT) and it combines both a-Si and c-Si materials. The literature reports that the efficiencies of the Sanyo HIT modules within the commercial sphere are currently higher than those of the commercial efficiencies for c-Si modules of the same rated power output. The Sanyo 250W HIT module, for instance, has a conversion efficiency of $18 \%$, while the SUNTECH $250 \mathrm{~W}$ c-Si module has an efficiency of $15.38 \%$ [26].

Furthermore, conversion efficiencies of $22 \%$ and $19.4 \%$ have been reported for the Panasonic 245W HIT cells and modules respectively, compared to the efficiencies of $14.8 \%$ and $15.1 \%$ reported for SUNTECH $245 \mathrm{~W}$ mono c-Si and poly c-Si modules, respectively [42]. Another hybrid technology is the microcrystalline $(\mu \mathrm{c}-\mathrm{Si})$ photovoltaic cell, which is manufactured through the hot wire chemical vapour deposition (HWCVD). This technology combines the c-Si and a-Si materials. It is designed to address the issue of Staebler Wronski effect in an ordinary thin amorphous silicon layer. Single $\mathrm{p}-\mathrm{i}-\mathrm{n}$ and $\mathrm{n}-\mathrm{i}-\mathrm{p}$ junction $\mu \mathrm{c}-\mathrm{Si}$ systems have achieved efficiencies of $8.9 \%$ and $9 \%$, respectively [45-48].

The other type of hybrid PV reported in the literature is the micromorph tandem cells. This technology combines a-Si and $\mu \mathrm{c}-\mathrm{Si}$ PV cells $[19,43]$. Though the hybrid PV 
modules have a relatively higher efficiency compared to the conventional c-Si modules, they are very expensive - one of the factors affecting their popularity and widespread application for electricity generation

\subsubsection{Organic and polymer solar photovoltaics}

The organic and polymer technologies are new in the PV industry. Like the CIS/CIGS technologies discussed earlier, they are in the developing phase [49-56]. Indications are that they have a relatively lower conversion efficiency of around 4-5\%. Apart from the fact that they are disposable and cost-effective, their production processes are not associated with high temperature and vacuum conditions. They are basically produced from thin film semiconductor materials. These are typically $100 \mathrm{~nm}$ of organic materials such as polymers and pentacene, polyphenylene vinylene, copper phthalocyanine, and carbon fullerenes.

\subsubsection{Dye-sensitized solar photovoltaics}

The photovoltaic process of this type of technology is made possible by a lightabsorbing or light-sensitive dye material, hence, its name dye-sensitised solar cell [49]. A typical dye material is essentially an iodide, and a nano-material regarded as titanium dioxide $\left(\mathrm{TiO}_{2}\right)$ that is usually used to hold the molecules of the dye material. The literature reports a conversion efficiency of $11 \%$ for dye-sensitised solar cells [50]. One of their challenges is that their system electrolyte contains unstable and potentially dangerous solvents. Such solvents are volatile and can spread through plastic material. This makes sealing of the organic cells to be very challenging

\subsubsection{Nanomaterial-based solar photovoltaics}

Though this technology is new in the solar PV parlance, it is expected to thrive in the future [50, 51]. Nanomaterials (e.g. nanowires and nanoparticles) have been classified as one of the third generation solar photovoltaic technologies [51-55]. They utilise nano-scale components and simultaneously manage the energy band-gap of the photovoltaic arrangements. This is brought about by the fact that the "quantum" limit effects of the nano-components allow an increase in the band gap of the technology. The devices that are used in nanotechnology-based photovoltaic cells include the carbon nanotubes (CNT), quantum dots (QD) and hot carrier (HC). They are briefly described as follows: 
- The CNT type is being produced by a hexagonal-lattice-carbon material with very good electronic and mechanical features. It has reached conversion efficiencies between $3 \%$ and $4 \%$ [51]

- The QD technology is developed by the combination of a group of semiconductor materials and is able to adjust the band-gap so as to increase the voltage or current. While the current output of this technology is increased by reducing the band gap, the voltage output increase is introduced by increasing the band-gap [52-54]

- The HC type uses the selective energy contacts to obtain light energy developed by the hot carriers, i.e. electrons and holes from the semiconductor material regions. This process is achieved without dissipating the excess energy as heat. Thus, it offers the possibility of achieving a solar cell with increased conversion efficiency [55]

\subsection{Solar photovoltaic model}

In order to characterise the performance of photovoltaic cells/modules or arrays, a well-established technique is usually employed, i.e. the equivalent circuit model of a solar cell. This particular model is introduced for the purpose of defining the I-V characteristics of the solar photovoltaic cell, module or an array, subject to some given operating conditions $[6,7,56-68]$.

\subsubsection{Single diode model}

A common equivalent circuit model in practice is the single-diode model. It primarily involves the arrangement of a current source, diode and a shunt resistance in parallel. This is then arranged in series with another resistance (i.e. series resistance), as shown in Figure 5. Also, in the I-V curve of solar cells, certain parameters are of interest such as the open-circuit voltage $\left(V_{o c}\right)$, short-circuit current $\left(I_{s c}\right)$ and the PV maximum power $\left(P_{m p p}\right)[6,7,56-69]$. The $P_{m p p}$ is expressed as the product of the maximum power point current $\left(I_{m p p}\right)$ and the maximum power point voltage $\left(V_{m p p}\right)$. 


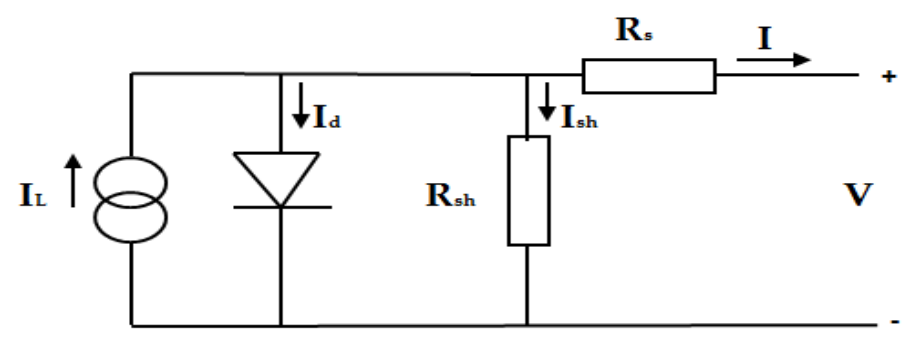

Figure 5: Equivalent circuit of a PV cell

By applying the Kirchoff's current law to the equivalent circuit model presented in Figure 5, the solar PV cell output current, I could be estimated by the following equation [56-69]:

$$
I=I_{l}-I_{d}-I_{s h}
$$

where:

$I$ is the output current of solar cell (A)

$I_{l}$ is the photo or solar-generated current (A)

$I_{d}$ is the diode current (A)

$I_{s h}$ is the shunt- leakage current (A)

The $I_{d}$ is modelled by employing the Shockley's ideal diode equation, as presented in the following equations:

$$
\begin{gathered}
I_{d}=I_{o}\left(\exp \left(\frac{V+I R_{s}}{n_{i d} V_{t}}\right)-1\right) \\
V_{t}=\frac{k T_{c}}{q} \\
I_{s h}=\frac{\left(V+I R_{s}\right)}{R_{s h}}
\end{gathered}
$$

By substituting Eqs. (2), (3) and (4) into Eq. (1), the output current of the solar cell is then represented by Eq. (5):

$$
I=I_{l}-I_{o}\left[\exp \left(\frac{V+I R_{s}}{n_{i d} V_{t}}\right)-1\right]-\frac{\left(V+I R_{s}\right)}{R_{s h}}
$$


where:

$k$ is the Boltzmann constant $=1.381 \times 10^{-23} \mathrm{~J} / \mathrm{K}$

$q$ is the electronic charge $=1.602 \times 10^{-19} \mathrm{C}$

$I_{o}$ is the diode reverse saturation current (A)

$n_{i d}$ is the ideality factor $(1 \leq \mathrm{n} \leq 2)$

$V_{t}$ is the thermal voltage $(\mathrm{V})$

$T_{c}$ is the absolute temperature $(\mathrm{K})$

$R_{s}$ is the series resistance $(\Omega)$

$R_{s h}$ is the shunt resistance $(\Omega)$

The single diode model is usually referred to as a five-parameter model because of certain parameters such as the $I_{l}, I_{o}, R_{s}, R_{s h}$ and $n_{i d}$ that are crucial for PV systems performance analysis. Such an analysis are useful for developing solar photovoltaic simulation models.

\subsubsection{Photovoltaic module model}

A solar PV module is made up of a specified number of series-connected cells, depending on the desired maximum power and voltage. Since the cells are arranged in series, the same current flows through them. This implies that the cell current is the same as the module current. However, the module voltage is obtained by multiplying the voltage of the cell by the total number of cells in the module arrangement. Eq. (6) represents the single diode model for a solar photovoltaic module or an array [6, 7, 5669].

$$
I_{m}=I_{l}-I_{o}\left(\exp \left(\frac{V_{m}+\alpha I_{m} R_{s}}{n \alpha V_{t}}\right)-1\right]-\frac{V_{m}+\alpha I_{m} R_{s}}{\alpha R_{s h}}
$$

where:

$V_{m}$ is the module voltage $(\mathrm{V})$

$I_{m}$ is the module output current (I)

$n$ is the ideality factor for the module

$\alpha$ is the number of modules

The I-V characteristics of a photovoltaic cell or module may be described as an "infinite or boundless" number of current and voltage operating points, at a particular 
set of conditions such as solar irradiance and ambient temperature. Figures 6 and 7 [6, 69, 216] are obtained from the PVSyst simulation software library and they show the behaviour of a solar module voltage and current with variations in the irradiance and temperature. While the solar irradiance is being varied between $200 \mathrm{~W} / \mathrm{m}^{2}$ and 1000 $\mathrm{W} / \mathrm{m}^{2}$, the temperature is varied between $10{ }^{\circ} \mathrm{C}$ and $70{ }^{\circ} \mathrm{C}$, in steps of $15^{\circ} \mathrm{C}$.

Figure 6 demonstrates that the PV module's current increases as the solar irradiance increases, while the voltage shows little increase. On the other hand, Figure 7 reveal that the module's voltage reduces as the temperature increases, while the current shows little or no increase with temperature.

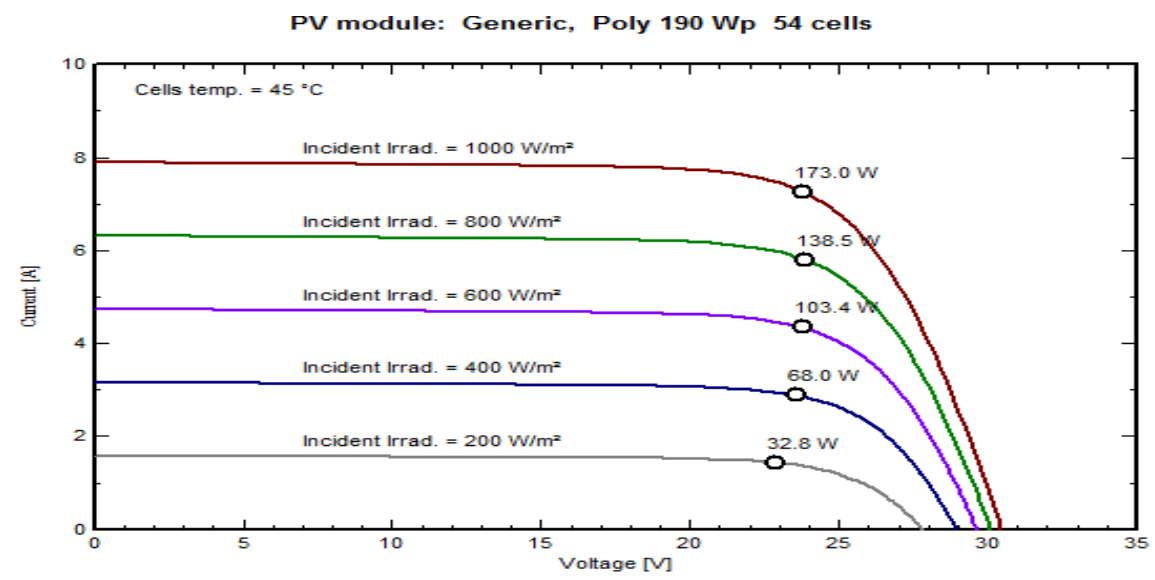

Figure 6: Module current and voltage at different irradiance levels

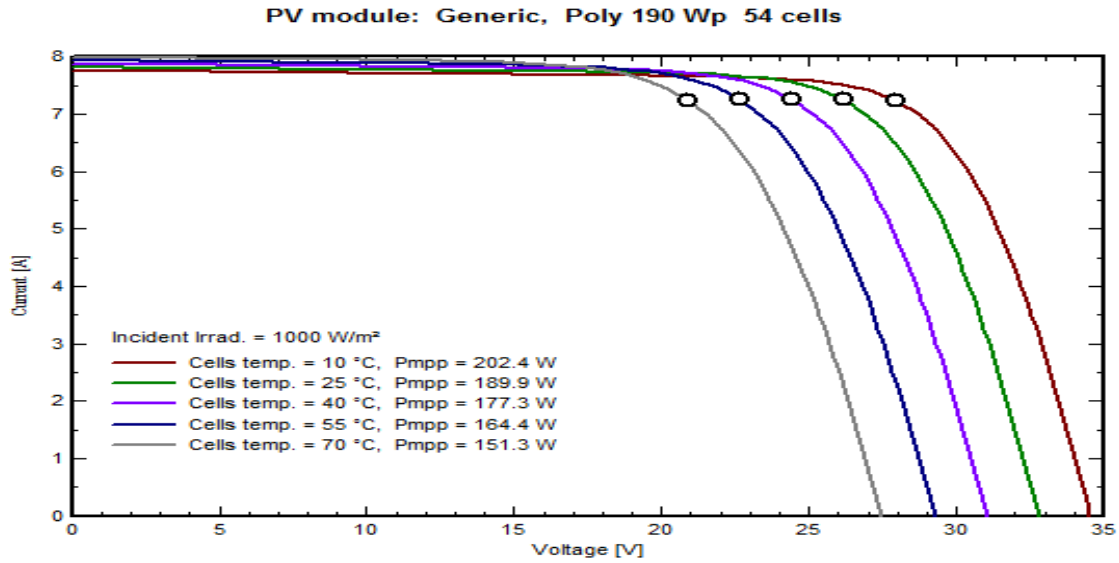

Figure 7: Module current and voltage at different temperature values 


\subsubsection{Fundamental solar PV terms}

The following are some basic terms in PV systems that are necessary for the understanding of the performance and analysis of solar electricity $[6,7,58-60]$ :

1) Solar PV device - this is used to convert solar energy into electricity. An example of this is the solar cell

2) Open circuit voltage $\left(V_{o c}\right)$ - essentially, this is the maximum value of DC voltage on the I-V characteristics. Such a value represents the operating point for a solar PV device at the no-load condition. It is usually obtained at the open-circuit condition (i.e. no load) when the values of current and power are zeros. Also, it is independent of the cross-sectional area of the solar cell or module, though it decreases as the cell or module temperature increases. The open circuit voltage for c-Si solar photovoltaics is usually in the range of $0.5-0.6 \mathrm{~V}$ at standard test conditions

3) Short-circuit current $\left(I_{s c}\right)$ - this represents the maximum value of DC current on the I-V characteristics. It is obtained at the short-circuit condition, i.e. when both the value of voltage and power are zeros. This electrical parameter has a linear relationship with the solar irradiance. For this reason, a location with good solar irradiation cycles will be suitable for solar electricity systems

4) Maximum power point current (Impp) - this is the value of electric current when a solar cell or module is being operated at the maximum power point, as shown in Figure 6. The maximum power current is usually obtained at a corresponding voltage value, $V_{m p p}$. According to the literature, the maximum power current of cSi technologies is usually expressed as $90 \%$ of the short circuit current (i.e. $I_{m p p}=$ $0.9^{*} I_{s c}$.

5) Maximum power point voltage $\left(V_{m p p}\right)$ - it is the value of voltage when a cell or module is being operated at the maximum power point, as shown in Figure 7. The maximum power voltage is usually obtained at a corresponding current value, Impp. The literature also reports that the maximum power point voltage has a typical value between $70 \%$ and $80 \%$ of the open circuit voltage

6) Maximum power $\left(P_{m p p}\right)$ - it is the product of the maximum power current and maximum power voltage. Therefore, it represents the power output of a solar cell or module when it is operated at the maximum power point. As shown in Figures 6 and 7, the maximum power output of the system corresponds to both 
$I_{m p p}$ and $V_{m p p}$. The maximum power point is the point on the "knee" of the I-V curve. It is always desirable that PV cells or modules are operated at the maximum power point. A process or method used to achieve this is the maximum power point tracking $(M P P T)$. Such a process could be achieved by a charge controller

7) Series resistance $\left(R_{s}\right)$ - this is one of the parameters of the single diode model that should be minimised to achieve optimum conversion efficiency of the cell or module

8) Shunt resistance $\left(R_{s h}\right)$ - unlike the series resistance, this parameter needs to be maximised to achieve optimum conversion efficiency

9) Module - this is usually referred to as an assembly of solar cells, i.e. the seriesconnection specified number of cells (e.g. 60 cells, 72 cells, etc.)

10) Panel - this is referred to as a group of solar modules

11) Sub-array - it is usually referred to as a part of a solar PV array

12) Array - it is essentially an arrangement of solar PV modules or panels together with the mechanical frame or support

13) BOS - the balance of system in PV systems is used to describe all the nonphotovoltaic components

14) Fill Factor - it may be defined as the ratio of $P_{m p p}$ to the product of $I_{s c}$ and $V_{o c}$, at standard test conditions. The literature reports that its typical values are usually over $70 \%$. Eq. (7) is used for calculating the $F F$

15) PV efficiency $(\eta)$ - it may be described as the ratio of $P_{m p p}$ to the product of the cell or module area and the solar irradiance at STC $\left(G_{s t c}\right)$, as shown in Eq. (8). While the cross-sectional area of the cell or module is usually expressed as the product its length $(l)$ and width $(w)$, the solar irradiance of $1000 \mathrm{~W} / \mathrm{m}^{2}$ is represented by Gstc. Furthermore, the denominator of Eq. (8), i.e. $l^{*} w^{*} G_{s t c}$ is usually regarded as the power density of the incident light

$$
\begin{gathered}
F \boldsymbol{F}=\frac{\boldsymbol{I}_{m p p} V_{m p p}}{\boldsymbol{I}_{s c} V_{o c}} \\
\eta=\frac{I_{m p p} V_{m p p}}{l \cdot w \cdot G_{s t c}}
\end{gathered}
$$




\subsection{Energy storage technologies}

In the previous chapter, it was established that one of the major challenges of renewable energy systems is the variability of their electrical outputs. In other words, renewable energies are weather-dependent and, due to this feature, they cannot be dispatched like the conventional energy systems (e.g. diesel power generators). However, energy storage systems are introduced into renewable electricity arrangements to mitigate the intermittency of their sources [14]. This allows increased and better use of renewable energy resources.

The aim of this sub-section is to present an overview of various energy storage technologies and their capability to balance the variability of renewable energy resources. The energy storage technologies for electricity applications (i.e. stationary applications) are classified as shown in Figure 8 [14, 70-101] and briefly discussed below.

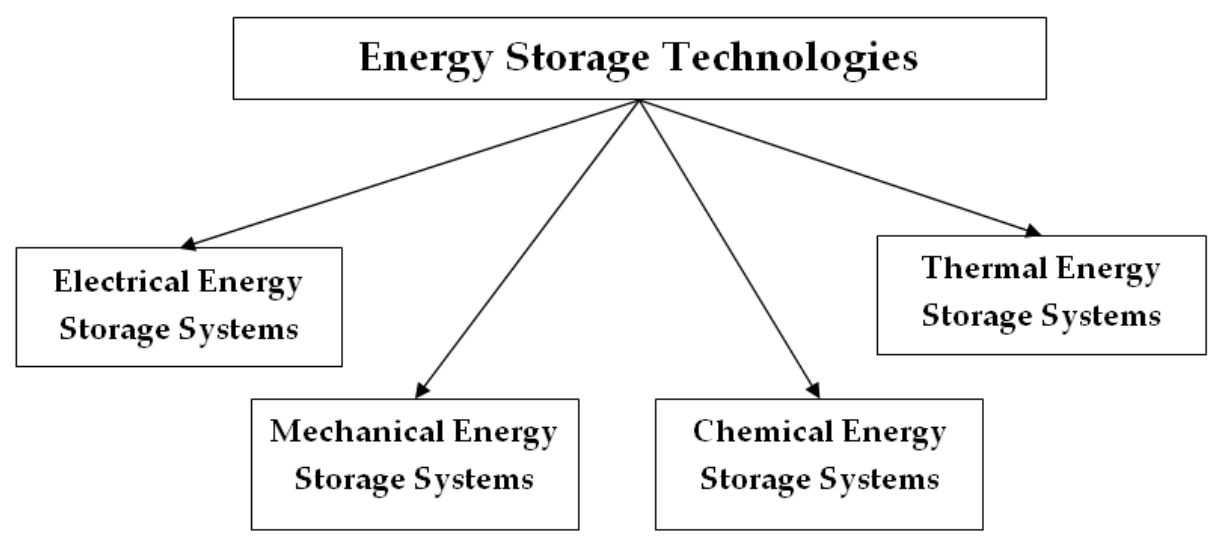

Figure 8: Classification of energy storage technologies

- Electrical Energy Storage Systems (EESS) include the Capacitor Storage (CS), Super-Capacitor Energy Storage (SCES) and Superconducting Magnetic Energy Storage (SCES) systems.

- Mechanical Energy Storage Systems (MESS) includes the Flywheel Energy Storage (FES), Compressed Air Energy Storage (CAES) and Pumped-Hydro Storage (PHS) systems

- Chemical Energy Storage Systems (CESS) includes the Battery Energy Storage (BES) system 
- Thermal Energy Storage Systems (TESS) includes the Aquiferous Thermal Energy Storage (ATES), Cryogenic Energy Storage (CES), Hot Thermal Energy Storage (HTES), Pumped-Heat Electrical Storage (PHES) systems

\subsubsection{Electrical Energy Storage Systems}

\section{1) Capacitors}

Capacitors have the capability to directly store electrical energy, and they can achieve this at a very fast rate. They have several thousand charge/discharge cycles compared to battery energy storage systems [70]. Also, they have been reported as having a capability for transient voltage stability in electrical power system applications. One major shortcoming of the conventional capacitor is its low energy density. This limitation has led to increasing research efforts toward the development of electrochemical capacitors, otherwise called supercapacitor energy storage (SCES) systems.

The SCES system has better electrical properties than the conventional capacitor, in terms of energy density and capacitance. These electrical properties are found to be thousands of times higher than the ordinary capacitors; this is because of their relatively larger surface area [70-73]. Even though the SCES system has higher power and energy densities compared to the ordinary capacitor (CS) system, its energy density is still comparatively lower than those of battery energy systems (e.g lead-acid battery). Therefore, capacitors generally have short discharge time and high self-discharge losses ( $20-40 \%$ per day) and these shortcomings limit them to electrical applications with relatively shorter timescales [71,74].

Some researchers have maintained that the power capacity of capacitors will be more appropriate for electrical applications, rather than their energy capacity [72, 75, 76-78]. This position implies that capacitors have higher power capacity than their energy density. While the round trip efficiency of the CS system is between $60 \%$ and $90 \%$, the SCES system has its value between $75 \%$ and $>95 \%$ [71, 73]. The CS and SCES systems have life spans of $\sim 5$ years and $>20$ years, respectively. In addition, the cycling capabilities of these two technologies are $>50,000$ and $>100,000$, respectively.

2) Superconducting magnetic energy storage

In the case of the SMES system, when a direct current (DC) is made to flow in its conducting medium, i.e. the superconducting coil, the electric energy does not "die out" 
as it does in the ordinary coil $[78,79]$. This unique property allows energy to be stored in a magnetic form until it is demanded when it will be converted back to electrical energy. The energy storage process of this system is achieved when the DC induces a magnetic field in the superconducting coils.

The SMES system has a fast response, with a switching time of around $17 \mathrm{~ms}$. Furthermore, it has a round-trip efficiency of about $98 \%$ [71, 78]. It also possesses a very good power density, just like capacitors, and has an expected life of over 20 years. Some of the major challenges of the SMES technology include short discharge time, high cost and the environmental issues of developing a strong magnetic field. The SMES system also has self-discharge losses and cycling capacity of $10-15 \%$ and over 100, 000, respectively [72, 80, 81]. It consists of the following components [71]:

- Superconductor (i.e. the coil): this is manufactured from an alloy, usually referred to as "niobium-titanium". Fundamentally, it operates at about $-269^{\circ} \mathrm{C}$, i.e. around the boiling point of liquid helium

- Cryogenic refrigerator: this uses helium as its refrigerant. Its main purpose is to maintain the operating temperature of the SMES technology

- Power conversion: this part is used for effective charging and discharging of the energy storage system. Electrical energy is stored by maintaining a positive voltage across the superconducting coils. On the other hand, energy is discharged by providing a negative voltage across the coils

- Control and management: this part is responsible for the control, coordination and the management of the flow of energy in the SMES system

\subsubsection{Mechanical Energy Storage Systems}

1) Flywheel energy storage

The FES system is an electromechanical system that allows electric energy to be stored in the form of kinetic energy. Its arrangement is usually made up a cylinder that rotates on magnetic bearings attached to an electrical machine [70, 71]. This machine operates as a motor during the charging process, while it behaves like a generator when energy is being discharged. During the charging process, i.e. when energy is being stored, the machine (electric motor) requires a very high speed to operate the flywheel 
system. Such a speed is about 10,000 r.p.m for low-speed rotors while it is between $>10,000$ and 100, 000 r.p.m for high-speed rotors [71, 74, 75].

When discharging energy from the FES system, the motor reverses and supplies energy through a suitable power converter. The FES system has a life span of about 15 years [81]. It apparently also has several thousand charge and discharge cycles compared to battery energy storage systems. It has a high round-trip efficiency, usually between $90 \%$ and $95 \%$. In addition, it has a cycling capacity of over 20,000 [70, 71]. However, like the capacitor energy storage systems, the FES technology has a short discharge time. Another shortcoming of the FES system is that it has high self-discharge and frictional losses, which can sum up to $100 \%$ /day.

\section{2) Compressed-air energy storage}

In the case of CAES system, energy is stored in the form of compressed air in dedicated underground reservoirs or chambers [82]. The energy that has been stored is later discharged when demanded, through a conversion process. Such a process involves combustion, so as to allow the operation of an expansion turbine to operate an electric generator. The heat energy developed when the system is charged, (i.e. compression of air), is usually released to the atmosphere during discharging (i.e. when the air is de-compressed) [71, 82]. A fuel is also usually required to reheat the air. This type of CAES system is regarded as the diabatic- CAES.

The CAES system has a round trip efficiency of around $85 \%$ with a life span between 20 and 40 years [71-75]. It is appropriate for large-scale energy storage applications in electrical power systems. Also, it has a cycling capacity between 10,000 and 30,000 and a small self-discharge loss. The shortcomings of the diabatic- CAES system are the requirement for an underground chamber or cavern; the waste of heat energy (i.e. the heat developed is dissipated to the atmosphere); the use of fossil fuels; and the release of pollutant emissions resulting from the combustion processes. However, research efforts to overcome the mentioned challenges have led to some developments in CAES system. The following technologies result from the improvements and modifications of the conventional diabatic-CAES system:

- Adiabatic-CAES: this technology is developed to exclude the use of fuel in the operation of a CAES system. It eliminates fuel consumption by storing the heat energy developed during the air compression process. The heat is then used to reheat the air during the expansion process. The combustion chamber in the 
conventional CAES is therefore replaced by thermal energy storage (TES) system. Adiabatic-CAES is scalable and eco-friendly [84-89]. It possesses hybrid storage characteristics (i.e. both the CAES and TES features) and has a relatively higher efficiency. The absence of combustors and fossil fuels is also an economic benefit of the adiabatic-CAES compared to the diabatic-CAES type. The first adiabaticCAES plant in the world, otherwise called the "advanced adiabatic-CAES (AACAES) demonstration plant", is planned to be commissioned in Germany by 2016. This plant is being developed by RWE Power and its collaborators, who aim to achieve an efficiency of about $70 \%$ [14]

- Isothermal CAES: this system also attempts to address some of the shortcomings of the diabatic and/or adiabatic-CAES system. It does not use fuel or thermal energy storage (TES) in its arrangements, but has the capacity to offer improved round trip efficiency (typically between about 70\% and 80\%) [86]. Furthermore, the system is also cost-efficient compared to the conventional CAES systems

- Small-medium scale CAES: suitable geological chambers or reservoirs are a requirement for large-scale compressed air storage systems. However, employing small-medium scale CAES systems will allow the use of artificial pressure vessels [89]. This way, the flexibility of operation can be achieved without using the underground reservoirs and thermal energy storage. This system is suitable for tri-generation applications, which includes decentralised energy generation and storage, air-cycle heating and cooling in a single CAES technology. Potentially, this could lead to significant use of small-scale energy systems and the reduction of greenhouse gas emissions in the future [14]

- Underwater/Ocean-CAES: this is another new storage system that does not require underground chambers. It is suitable for offshore renewable energy systems. Recently, Hydrostor (a company situated in Canada) has patented an energy storage technology that employs semi-adiabatic Underwater-CAES (UWCAES) $[14,84,88,89]$. Such a system can store a large-scale energy for about 4 48 hours, for systems between 1 and $50 \mathrm{MW}$. The UW-CAES is expected to lead to a widespread application of microgrids and distributed energy resources in the future 


\section{3) Pumped-hydro storage}

This system has been reported as a well-developed, low cost and widely used option for large-scale energy storage applications in electrical power systems [14]. Its arrangement usually has double interconnected reservoirs at different heights (i.e. lower and upper caverns), water pumping mechanism to store water to the upper reservoir and a turbine to produce electricity when water is released into the lower chamber. The PHS has a round-trip efficiency of $70-80 \%$, with a life span ranging from 40 to 60 years [71,72,75,76]. It has a cycling capacity of 20,000 - 50,000; it also has a very small self-discharge loss per day.

The main challenges of the pumped hydro technology are the requirement for an appropriate site (usually a dedicated land mass), high capital investments, long time of construction, and the environmental issues [71]. However, new research and development efforts are devising means of reducing the environmental issues of the pumped hydro system. These efforts have led to the development of systems such as the sub-sea, seawater, and variable-speed pumped hydro energy storage technologies.

- Sub-surface PHS: in this system, either the lower or upper chamber or both chambers is located at the sub-surface (i.e. below the ground level) [90]. There is also the possibility of using existing subsurface structures for economic reasons. An example of the sub-surface PHS is the Elmhurst Quarry system in Illinois, US, which is proposed to use an abandoned mine and quarry for both the lower and upper reservoirs. This system has a size between 50 and 250MW and a storage capacity of 708.5GWh, according to the literature $[14,71]$

- Seawater PHS: in this system, the ocean is used as the lower reservoir. Less land is therefore used (i.e. only one reservoir is needed on land). It could be developed near to renewable power generation plants viz. the wind and solar [70, 71, 91, 92]. Such a system promises a widespread application of renewable energy resources in the future. The only seawater PHS in the world that uses the seawater is the Okinawa Yanbaru plant, situated in Japan. It has an installed capacity of 30MW, with the Pacific Ocean serving as its lower reservoir, while the upper reservoir is an artificial device [14]

- Variable-speed PHS: one of the shortcomings of the conventional fixed-speed water pumping turbines used around the globe is their inability to provide 
frequency regulation to the electrical system [93-96]. This is due to their fixedspeed. However, variable-speed turbines have the potential to address this shortcoming, thus, offering relatively better performance

\subsubsection{Chemical energy storage system}

\section{1) Batteries}

They are the most widely used energy storage systems. Examples are lead-acid, nickel cadmium (Ni-Cd), nickel metal hydride (Ni-MH), lithium-ion (Li-ion), sodium sulphur (NaS), vanadium redox (VRB), sodium-nickel chloride (ZEBRA) and zinc bromide $(\mathrm{ZnBr})$ [71]. These systems have the potential to respond to electrical load changes within $20 \mathrm{~ms}$ and they have a round-trip efficiency ranging from 60 to $90 \%$ [14, 71,97]. Furthermore, of the mentioned batteries, NaS, VRB, ZEBRA and $\mathrm{ZnBr}$ are referred to as high- energy batteries because they have a relatively higher storage duration timescale.

This thesis uses the battery energy storage system as one of the major components of the technical solutions. Therefore, emphases are laid on the different types of battery technologies that are in existence and their suitability for the development of the solar photovoltaic microgrids, on which this thesis concentrates [14, 70-81, 97-102].

- Lead-acid: this technology is the oldest and most widely used type of rechargeable battery energy storage systems. It was invented in 1859 by a French physician called Gaston Planté. It usually comprises of connected cells, an electrolyte, and the electrodes (i.e. positive and negative conductors) [70-71, 101]. There are primarily two kinds of the lead-acid battery system that are available in practice. These systems are the flooded or vented, and the valve-regulated or sealed types. While the flooded technology is a lead acid battery that requires frequent water replacement to forestall the battery's electrolyte drying, the valveregulated technology does not require water replacement. The valve-regulated battery is classified into "Absorbed" and "Gelled" electrolyte types with relatively higher costs. The lead-acid is currently the most commonly used energy storage system for renewable energy applications. It has a cycle life of about 2,000 - 2,500 cycles, a round-trip efficiency of $70-90 \%$ and a life span of 5 15 years $[14,71,72,78,97]$. It also has a low self-discharge loss of $0.1-0.3 \% /$ day. 
However, this battery system is characterised by a low cycling capacity and also has a negative environmental impact because of the production of toxic remnants

- Lithium-ion: this is not only recognised in the electronics field, but is also wellknown in the transportation industries [71]. It has low self-discharge losses of $0.1-0.3 \% /$ day. It has a higher cycling capacity and a round trip efficiency of about 10,000 and $100 \%$, respectively, compared to all other battery energy systems [70, $71,74,76]$. It also has a life span of 5 - 15 years. However, it is still currently expensive compared to the conventional lead-acid storage technology

- Nickel cadmium: there are basically five different battery technologies that use the nickel electrode material. These technologies are nickel-zinc (Ni-Zn), nickelhydride (Ni-H2), nickel cadmium (Ni-Cd), nickel metal hydride (Ni-MH) and nickel-iron (NiFe). While the $\mathrm{Ni}-\mathrm{Cd}$ and $\mathrm{Ni}-\mathrm{MH}$ technologies are more popular than the other alloys, the $\mathrm{Ni}-\mathrm{Cd}$ has been identified as the most widely used nickel-electrode battery technology in the industry [71]. It has a life span of 10 20 years. The shortcomings of the Ni-Cd technology include low cycling of $\sim 2,000$ - 2,500, and the adverse environmental impact of generating cadmium [14, 71]. It has a slightly higher self-discharge losses of $\sim 0.2-0.6 \%$ than the lead -acid batteries

- Metal-air: this battery system is a form of fuel cell technology that uses a metal as fuel while the air serves the purpose of an oxidising agent. It is eco-friendly and can also offer cost-efficient energy storage in the future. However, it possesses a low round trip efficiency (usually less than 50\%) and a poor recharging capability [70, 71]. Its cycling capacity is between 100 and 300, even though it has very small self- discharge losses

- VRB: in the case of vanadium redox battery technology, energy is being stored through the transference of electron between dissimilar ionic vanadium materials $[70,71]$. It possesses a cycling capacity of $>12,000$ and small self-discharge loss per day. It has a life span ranging from 5 to 10 years, with a round-trip efficiency of over $85 \%[14,71]$. However, because it produces toxic remnants, it has an adverse environmental impact

- $\mathrm{ZnBr}$ : this is also a high energy technology. It presents a relatively higher performance compared to the conventional lead acid batteries [98]. It has a life 
span of 5 - 10 years, cycling capacity of over 2,000, a round-trip efficiency ranging from 72 to $85 \%$, and a small daily self-discharge loss [71, 98]

- NaS: studies indicate that this technology possesses a high power and energy density, of about four times more than those of lead-acid battery systems. The battery system operates at a temperature ranging from 300 to $350{ }^{\circ} \mathrm{C}$, and this is one of its major shortcoming [71]. This is because an external heating arrangement is a requisite for its efficient operation. It has relatively higher selfdischarge losses of about $20 \%$, a cycling capacity of about 2,500 and a life span 10 to 25 years [71, 80,81]

- ZEBRA: it is another high-energy battery technology, which possesses a high operating temperature property of $\sim 300^{\circ} \mathrm{C}$. Its electrodes (i.e. cathode and anode conductors) are nickel chloride and liquid sodium, respectively [71]. It has a cycling capacity of over 2,500 and self-discharge losses of $\sim 15 \% /$ day. In addition, this technology possesses a lower energy and power density compared to the $\mathrm{NaS}$ battery systems. Its life span ranges from 10 to 14 years. One of the environmental benefits of the ZEBRA batteries is that they could be recycled and developed into new batteries

\subsubsection{Thermal energy storage system}

This system employs materials that can be kept at high or low-temperature in containers with well-designed or dedicated insulators [14]. Electricity is then generated from the heat or cold that is developed from this process, through a heat engine system. This storage technology could be classified into the low- and the high-temperature storage systems. However, their modus operandi is determined by the operating temperature of their storage media, in relation to the room temperature. Systems having a temperature below $-18{ }^{\circ} \mathrm{C}$ are used for industrial cooling applications [71, 74, 76]; those having a temperature ranging from 0 to $12{ }^{\circ} \mathrm{C}$ are used for achieving cooling in buildings; those with a temperature of $25-50{ }^{\circ} \mathrm{C}$ are employed for heating in buildings, while systems with temperatures that are greater than $175{ }^{\circ} \mathrm{C}$ are used for industrial heat storage applications.

1) Low-temperature TES: this is categorised into aquiferous (ATES) and cryogenic (CES) thermal storage systems. The ATES is usually associated with the process involving the use of a refrigerator to cool or ice water. Such a process could be 
useful to users when there are some cooling demands [71]. The CES is associated with the process of boiling cryogen (i.e. liquid nitrogen) by the heat of the surroundings. The heated cryogen is then used for producing electricity through cryogenic heat engine arrangements. The CES system has a life span ranging from 20 to 40 years, a self-discharge loss of $0.5-1 \%$ and a round-trip efficiency ranging from 40 to $50 \%$

2) High-temperature TES: a good example of this system in practice is the solar thermal electric power plant. Such a plant stores heat energy in a double tank of molten salt. Apart from molten salt, the other potential materials for the hightemperature TES include concrete, phase change materials (PCMs), saturated steam and highly pure graphite material [71]. The high-temperature TES has a life span of 5 - 15 years and self-discharge losses ranging from 0.05 to $1 \%$. An example of the solar thermal power plant is the Solana Generating Station in the US. It has a capacity of $280 \mathrm{MW}$ and uses the molten salt to store energy for 6 hours [94]. The pumped heat storage technology is another form of thightemperature TES and is in its development stage. It has an expected round-trip efficiency of $75-80 \%$ [14, 71]. In addition, its design allows a scalable and costeffective storage arrangements compared to the pumped hydro system. It will also have a less environmental impact compared to the pumped hydro technology

\subsection{Review of related works}

Several studies have been reported on renewable energy systems for off-grid applications, especially in the rural and isolated areas in developing countries, within and outside Nigeria. Such research is based on using the available renewable and nonrenewable energies (particularly solar, wind, diesel/petrol sources) in different regions or communities of different countries to propose energy supply systems. The authors report different energy scenarios, and these are reviewed in this section.

A study has been presented on the household electrification system based on solar photovoltaic technology for rural development in Nigeria [103]. The research primarily assesses domestic load demand as a way to propose suitable photovoltaic systems for the off-grid homes. It considers light bulbs, TVs and radios as basic domestic appliances for those living in the rural/remote areas, using four communities 
in the Southwestern Nigeria as case studies. Each community has less than 200 houses. The authors obtained data on domestic load for the communities through interviews. Rural homes in Nigeria are reported to require a daily energy supply of $2.324 \mathrm{kWh}$ (i.e. $850.8 \mathrm{kWh} / \mathrm{yr}$ ). This work presents information about the energy consumption of the specified off-grid users, and it may be useful for planning. However, while the energy consumption and of course the load profile of the users is an important aspect of energy system development, the study does not consider or propose technical solutions for the communities. Nor are detailed sizing methodologies for solar PV, batteries and inverters/charge controllers are discussed in the research. Such aspects are vital parts of the development of the energy system for off-grid areas that need to be considered.

Furthermore, a research project has been discussed that concentrates on the prospect of solar electric power supply to remote areas in Nigeria [104]. The study focuses on the economic analyses of PV system compared to different energy options viz. diesel generator and the grid systems. The work's main contribution is that it finds the photovoltaic system that has the least yearly cost of energy supply, amongst the considered energy options. However, while this gives a general indication of the costeffectiveness of the solar PV system, the research does not consider a detailed technoeconomic analysis that is needed to make a strong case for renewable energy systems compared to other technologies.

Photovoltaic technologies for distributed electric power supply are discussed in [105]. This study, which focuses on Nigeria, presents the status and the possibility of a solar photovoltaic system for remote locations and telecommunication systems. It reports some of the existing PV projects in the country and suggests policies that could be helpful for widespread use of solar power systems in the isolated communities in Nigeria. The research provides useful information for understanding the status of solar PV systems in Nigeria. However, it does not present the technical and economic evaluations that are of interest for developing energy systems for remote areas.

In addition, a research was conducted on rural area power supply in Nigeria, so as to compare the costs of the PV, diesel/gasoline and the utility systems [106]. This study presents the sizing methodology for the photovoltaic system and applies life cycle cost analysis to determine the costs of the different energy options. The research concludes that the solar PV system has a potential for achieving cost-effective energy 
supply, compared to the other options. However, the battery state of charge and load growth analyses were not considered; nor is the effect of temperature on the PV system evaluated.

A study has also been reported, with a focus on the electricity access in Nigeria [107]. It emphasises the viability of off-grid solar photovoltaic systems. It evaluates the life cycle cost of an off-grid solar photovoltaic electrification and then compares it with the electricity grid option, using the RETScreen tool. A remote community of 40 households in the northern part of Nigeria was used as a case study. In addition, an initial electric load of $5 \mathrm{~kW}$ was considered. The research concludes that the PV project will not be economically viable over the 25 -year project life, compared to the grid supply tariff of $22 / \mathrm{kWh}$ (i.e. about US $\$ 0.15$ per $\mathrm{kWh}$ ). It is recommended that government support will help to make PV projects viable in the country. The study does not provide detailed engineering analysis, which is a vital aspect of energy planning and development.

The assessment of the cost-effectiveness of solar photovoltaic systems for telecommunication applications in Nigeria is presented in [108]. The results of the research show that a solar PV has the potential to reduce the cost per unit energy supply for telecommunication systems. Furthermore, a paper is presented that focuses on how $100 \%$ renewable electricity supply systems could be possible in Nigeria [109]. It concentrates on the country's renewable energy potential and emphasises the possibility of achieving electricity generation totally based on renewable energy resources. The work does not provide the technical solutions for Nigeria, even though it presents the abundant renewable energy available. Although an analysis of the available energies in the country is informative, it still does not solve the energy problem of the off-grid communities. People's energy needs need to be assessed, which can inform the solutions required to address the energy shortage challenges.

The optimal sizing of hybrid energy system for a remote telecommunication tower has also been discussed, using Nigeria as a case study [110]. It employs the Hybrid Optimization Model for Electric Renewables (HOMER) tool to simulate different energy configurations such as the PV/diesel generator, PV/Wind turbine/diesel generator and diesel generator only. It focuses on the technical and economic assessment of the energy options, and also compares the amount of carbon emissions produced by the systems. 
The peak load and average demand per day of the telecommunication system are 3.3 $\mathrm{kW}$ and $37 \mathrm{kWh}$ respectively. The sizes of PV, diesel generator, and wind turbine are 8 $\mathrm{kW}, 5.5 \mathrm{~kW}$, and $1 \mathrm{~kW}$, respectively. The research results reveal that the energy configurations that incorporate renewable energy systems offer the most economical solutions. The renewable energy systems also contribute to the reduction of carbon emissions.

A paper on the assessment of decentralised hybrid PV-diesel power system for the northern part of Nigeria has been published [111]. The authors also employed HOMER for analysing a system with daily load demand and daily peak load of 1,500 $\mathrm{kWh}$ and $236 \mathrm{~kW}$ respectively, for rural/isolated communities of around 1,500 households. Each home is assumed to consume $1 \mathrm{kWh}$ of electricity per day. The results of the simulation present configuration 1 (i.e. PV and two diesel generators with the rating of $175 \mathrm{~kW}, 160 \mathrm{~kW}$, and $100 \mathrm{~kW}$ respectively), as one having the least total net present cost and cost of energy. The system also produces relatively lower carbon emissions.

The research discussed in [110, 111] presents simulation models for different energy options - PV/diesel compared to a diesel generator system. However, the authors do not provide information about the battery state of charge analysis, which is an important aspect of the technical analysis. The design/sizing methodologies are not included in the papers, nor is the impact of losses on the PV system's performance discussed. In addition, they do not analyse the reliability, load growth and the life cycle impact of their proposed solutions.

An economic evaluation of hybrid energy systems for rural electrification has also been discussed in [112]. This research uses Nigeria's six geopolitical zones as case studies. The authors use HOMER to simulate different energy systems for six different areas, each from a geopolitical zone in the country. The energy systems that were simulated include PV/diesel/battery, PV/wind/diesel/battery, wind/diesel/battery, PV/diesel, PV/wind/diesel and wind/diesel. The analysis results show that the $\mathrm{PV} /$ diesel/battery system has the least total net present cost and cost of energy. The carbon emissions were minimised to about $23 \%$ by including renewable energy systems in the energy mix, e.g. $13,604 \mathrm{~kg} / \mathrm{yr}$ compared to the base case of about $60,000 \mathrm{~kg} / \mathrm{yr}$. This research also presents a techno-economic analysis of different electricity options 
for the remote homes, but it does not consider the battery energy system analysis and the effect of losses on the PV system. The work also does not consider the reliability, load growth and the life cycle impact of the proposed systems.

The feasibility of renewable energy resources for water pumping in sub-Saharan Africa was presented in [113], using central Nigeria as a case study. The paper employs a techno-economic analysis to investigate whether or not renewable energy systems are able to compete with the conventional option for pumping clean water. The results of the study indicate that even though the initial capital investments of renewable technologies are high, their "increased" upfront costs are lower than the ongoing fuel costs of the conventional system. This signifies an economic benefit of the renewable energy systems. However, detailed methodologies and technical analysis of the PV system are not considered in the study. Such consideration is crucial to the better understanding of the operation of the system.

The simulation of off-grid generation options for remote villages in Cameroon was also discussed [114]. This research conducts the techno-economic analysis for different hybrid electricity configurations by using the HOMER software. The systems that are considered are Micro hydro/LPG generator/battery, Micro hydro/diesel generator/battery, PV/LPG generator/battery and PV/diesel generator/battery. The sizes of the micro hydro, LPG generator and PV systems are $14 \mathrm{~kW}, 15 \mathrm{~kW}$, and $18 \mathrm{kWp}$ respectively, to support daily demand and peak load of $110 \mathrm{kWh}$ and $12 \mathrm{~kW}$ respectively. The results of the simulation show that the micro hydro/LPG generator option with a battery capacity of $36 \mathrm{kWh}$ generated energy at the cost of $0.296 €$ per $\mathrm{kWh}$ for the remote community while the PV/LPG generator with a battery capacity of $72 \mathrm{kWh}$ produced energy at the cost of $0.576 €$ per $\mathrm{kWh}$. The authors posited that these results indicate the possibility of renewable energy systems becoming a suitable alternative option for electrifying isolated areas. However, the analysis of the system reliability and load growth were not discussed. Nor was a detailed engineering design and analysis provided.

The design of a photovoltaic-wind hybrid power generation system was also presented in [115], focusing on remote areas of Ethiopia. The system was simulated in HOMER environment, considering the basic appliances such as light bulbs, water pumping machine, radio receiver, television, health vaccine refrigerators and school 
appliances. The total daily load demand of the community is about $78 \mathrm{kWh}$. The research output indicates that the energy configuration with a renewable fraction of $84 \%$ produces the least total net present cost and cost of energy. The authors indicated that renewable energy systems are able to supply the entire users' load demand requirements in the remote communities. The research does not consider PV energy losses, battery state of charge, system reliability, load growth and the life cycle impact analyses.

A paper on the feasibility of pico-hydro and photovoltaic hybrid power systems for remote villages in Cameroon has been published [116]. HOMER simulation software was used to model the energy systems for daily load demand and peak load of $73 \mathrm{kWh}$ and $8.3 \mathrm{~kW}$, respectively. The study does not consider energy losses, battery state of charge, reliability, load growth and the life cycle impact assessments.

Furthermore, an overview of solar photovoltaic systems for decentralised rural electrification was presented in [117]. The study highlights the experiences of electrifying rural and remote areas. It also discusses the obstacles, institutional structures, and the financial requirements. The paper's techno-economic aspects concentrate on the system design techniques and approaches. These include the performance analysis, comparison of the techno-economic design of different energy configurations and the environmental implications. This work provides an insight into the challenges facing the off-grid energy systems and possible ways of planning such systems using India as a case study.

An analysis of different solar photovoltaic power systems to determine optimal PV panels and system installation has been presented, with a focus on Kahramanmaras in Turkey [118]. The research makes a comparison between the performance of three different PV technologies, namely mono c-Si, poly c-Si and thin film, with each of the systems having a power rating of $3 \mathrm{~kW}$. The results of the study present the poly c-Si modules as the optimal technology for the specified area, in terms of annual incomes and breakeven point.

The design and economic analysis of an off-grid PV system for household electrification has been discussed [119]. The paper focuses on a single household application in Faisalabad, Pakistan that requires $1.928 \mathrm{~kW}$ of solar PV to support an 
average daily demand of $5.9 \mathrm{kWh}$. The results indicate that the system's life cycle cost and the cost of energy are lower than those of conventional systems. However, the study also does not consider energy losses, battery state of charge, reliability, load growth and the life cycle impact analyses.

The study of a hybrid distributed renewable electric power generation for rural areas was discussed in [120], using Bangladesh as a case study. The authors used HOMER to model off-grid systems (PV/biomass) for two villages of 900 and 700 houses. The daily load demand and peak load of village 1 are 3,100 $\mathrm{kWh}$ and $394 \mathrm{~kW}$, respectively, while the corresponding values for village 2 are 2,400 kWh and $368 \mathrm{~kW}$. Results indicate that village 1 will require $500 \mathrm{~kW}$ PV and $120 \mathrm{~kW}$ biomass plant, while village 2 will require $200 \mathrm{KW}$ and $100 \mathrm{~kW}$ of PV and biomass systems respectively. The total net present costs and cost of energy for the renewable energy-based systems of the two villages are found to be lower than furnace-oil fuelled power plant in Bangladesh. The study discusses the techno-economic analysis of the PV/biomass system. However, it does not include analysis of losses of the proposed energy systems. The research does not also consider the battery state of charge, reliability, load growth and life cycle impact analyses.

The optimum utilisation of renewable energy sources in a remote area of India was discussed in [121]. The research presents the analysis of the potential of different renewable energy systems such as PV, wind and biomass before developing an algorithm to evaluate the optimum values of the resources. Though the results of the work are informative and will be useful for optimisation tasks, the work does not include evaluation of losses, battery state of charge, load growth and the life cycle impact.

The options for off-grid electrification in the kingdom of Bhutan was presented in [122]. The HOMER simulation software was used for modelling energy systems, both for off-grid and grid-connected applications. The authors attempt to find the costeffective energy configuration for different kinds of available renewable energy resources. This leads to four different systems: PV/diesel, PV/wind/diesel, PV/battery and wind/diesel. The system's average load demand is $12.12 \mathrm{kWh} /$ day. Results reveal that PV/diesel hybrid and PV/battery systems could be competitive options for remote areas, rather than considering the grid extension option. However, the authors do not 
consider energy losses, battery state of charge, reliability, load growth and the life cycle impact assessments.

A review of the techno-economic feasibility analysis of solar PV system is presented in [123]. The paper discusses different existing works on PV power generation system for off-grid electricity applications. It highlights the system design methods, techno-economic analysis, performance analysis and life cycle analysis, thus, providing useful background and insights into solar photovoltaic applications for isolated areas.

Additionally, the new sizing method for solar photovoltaic water pumping systems has been discussed [124]. The method presented in the study considers the loss of power supply probability. It also applies life cycle cost analysis to evaluate the system's cost. The paper is found useful for understanding how reliable solar PV energy system could be. The performance analysis of off-grid PV electrification systems for sustainable information and communications technology (ICT) development has also been presented [125]. The research discusses a field study in four different regions of Thailand and quantifies the amount of energy that could be delivered by a PV system for ICT application. However, the research does not include the battery state of charge, reliability, load growth and the life cycle impact assessments.

The optimal sizing of a PV/wind/diesel/battery system for an off-grid region has also been discussed, using Rafsanjan as a case study [126]. The research employs a harmony search approach for optimising the performance of the proposed energy system, which will be useful for solving optimisation problems. However, the work does not consider the battery energy, reliability and life cycle impact analyses, which are crucial for off-grid energy system design and planning. In addition, a study has been discussed that focuses on the performance evaluation of an off-grid solar PV system [127]. The paper uses Saudi Arabia as a case study. The paper does not consider reliability, load growth, and the life cycle impact evaluations.

The wind/PV/battery hybrid system application was discussed, using Bangladesh as a case study [128]. The authors designed $27 \mathrm{~kW}$ PV and $39 \mathrm{~kW}$ systems for the daily demand and peak load of $169 \mathrm{kWh}$ and $61 \mathrm{~kW}$, respectively. This hybrid system is also compared with the PV/battery system. Results reveal that the wind/PV 
hybrid system has an optimal total net present cost and cost of energy, compared to the $\mathrm{PV} /$ battery option. This is due to the relatively higher renewable energy fraction of the hybrid configuration. The study presents the system's techno-economic design, but it does not consider the losses, battery energy, reliability, load growth and the life cycle impact analyses.

The various indicators for the life cycle analysis (LCA) of residential photovoltaic systems were compared in [129]. The study discusses the global warming potential (GWP) of $1 \mathrm{kWh}$ of electricity generated from solar PV systems, using Europe as a case study. The authors concluded that the greenhouse gas (GHG) emissions per $\mathrm{kWh}$ electricity produced from silicon crystalline photovoltaic systems range from 50 to $100 \mathrm{~g}$ $\mathrm{CO}_{2}$-eq. They further indicated that the lower values are valid for Spain and Italy because of their relatively higher solar irradiation. However, the higher values were obtained for Belgium, Germany and the United Kingdom with lower solar irradiation.

Additionally, the life cycle energy and environmental comparative analysis of solar PV systems has been discussed [130]. The research lays emphasis on the domestic and overseas manufacturing scenarios of silicon-based solar photovoltaics. The authors argued that current LCA research works are limited to North America and Europe, while as of today, most of the solar photovoltaic modules are produced in the nonOECD nations, e.g. China. Three different PV technologies were investigated, viz. mono c-S-, poly c-Si, and ribbon c-Si. The paper reports that the life cycle carbon emissions of these modules manufactured in China are 72.2, 69.2 and 54.3g $\mathrm{CO}_{2}$-eq. per $\mathrm{kWh}$, respectively. The results presented in $[129,130]$ provide useful information and methodology for assessing the proposed SPMs' life cycle environmental performance.

The contributions of these existing studies form a useful background for understanding renewable energy-based electricity systems. However, the review makes it clear that the majority of the research does not consider solar photovoltaic energy losses, battery state of charge, reliability, and load growth analyses. Furthermore, most of the existing publications estimate the environmental performance of the photovoltaic systems with the assumption that they are 100\% carbon neutral. Such studies do not consider the energy flow and the amount carbon dioxide emitted over the system's life cycle. These knowledge gaps will be addressed by this thesis. 
This thesis introduces the design and modelling approaches based on the global engineering standards and practical experience. It also presents the load flow analysis showing the relationship between generation and load demand, including the evaluation of the unmet demand, loss of energy supply probability and the availability indices. It further evaluates the proposed energy system's economic and the life cycle environmental impact. The results of the thesis are expected to add value to the existing body of knowledge. Such knowledge can be useful for planning solar photovoltaic power systems in Nigeria, including other developing countries.

\subsection{Relevant global engineering standards}

\subsubsection{IEEE standards}

1) IEEE standard 1562 [131]: this is basically a guide for array and battery sizing in stand-alone PV systems. It is developed to assist PV system designers, engineers or consumers in sizing off-grid solar photovoltaic systems, by using the peak or rated sun-hour method. It gives recommendations on how to use the worst-case scenario, (i.e. usually the worst monthly solar irradiation and the highest users' load demand) to determine a suitable photovoltaic power system

2) IEEE standard 1013 [132]: this presents a recommended practice for sizing lead acid batteries for stand-alone PV systems. It discusses a method for determining the capacity of both vented and valve-regulated lead-acid battery systems for offgrid PV systems application. It also highlights the installation, maintenance, safety and testing procedures for such battery systems

3) IEEE standard 1361 [133]: this concentrates on the selection, charging, test and the evaluation of lead-acid batteries used in stand-alone photovoltaic systems. The off-grid PV system parameters (e.g. charging) and the operating conditions are presented in light of the characteristics and performance of the battery system

4) IEEE standard 1561 [134]: this serves as a guide for optimising the performance and life of lead-acid batteries in remote hybrid power systems. It is relevant for considering lead-acid batteries for off-grid hybrid energy supply system

5) IEEE standard 1661 [135]: this is a guide for testing and evaluating lead-acid batteries that are employed in photovoltaic hybrid power systems. It is relevant to PV/diesel hybrid systems or any other hybrid system involving at least one renewable energy technology 


\subsubsection{IEC standards}

1) IEC standards 61724 [136]: this concentrates on the photovoltaic system performance monitoring- guidelines for measurement, data exchange, and analysis. This standard basically recommends methodologies for evaluating PV system's electrical performance in relation to the operating conditions

2) IEC/TS 62257-9-1 [137]: this provides technical specifications and recommendations for small renewable energy and hybrid systems for rural electrification systems. It focuses on micropower systems. Such systems are designed and modelled for isolated dwellings or communities or villages. The standard provides suggestions on how to select the right system for the right locations and the possible scenarios

3) IEC/TS 62257-2 [138]: this presents recommendations for small renewable energy and hybrid systems for rural electrification. Its main emphasis is on how to develop a range of electricity systems from specified energy demand requirements. The standard presents methodologies that could be useful for evaluating the socio-economic aspect of decentralised energy projects

4) IEC/TS 62257-4 [139]: this presents technical specifications and recommendations for small renewable energy and hybrid systems for rural electrification, focusing on system selection and design. It emphasises the assessment of the users' needs and also highlights different electrical power systems or architectures that can be employed for meeting the specified needs

5) IEC/TS 62257-7 [140]: this presents technical specifications and recommendations for small renewable energy and hybrid systems for rural electrification, concentrating on conventional generators. In particular, it specifies the general requirements for generators of up to the rated maximum power of $100 \mathrm{kVA}$ in distributed electricity systems for remote communities. It considers the system's components selection, sizing, installation and the operation and maintenance $(\mathrm{O}$ and $\mathrm{M}$ )

6) IEC/TS 62257-7-1 [141]: this presents technical specifications and recommendations for small renewable energy and hybrid systems for rural electrification, concentrating on solar PV generators. It proposes technical specifications for the design and development of small-scale solar PV generators of up to $100 \mathrm{~kW}$-p for off-grid applications in isolated areas 
7) IEC/TS 62257-7-3 [142]: this discusses technical specifications and recommendations for small renewable energy and hybrid systems for rural electrification. It focuses on the selection of generator set for off-grid locations. It is also an addition to IEC/TS 62257-7

8) IEC/TS 61836 [143]: this basically presents the terms, definitions, and symbols of solar PV energy systems. Such symbols and terms are recognised in the field of solar PV practice

\subsection{Chapter summary}

The chapter has provided a brief overview of the renewable energy systems, with information on the current renewable electricity proportion of the total global electricity capacity. It reports that renewable energy production accounts for $22.8 \%$, while the remaining $77.2 \%$ accounts for the conventional energy resources - fossil fuels and nuclear. The total worldwide electricity generation at the end of 2014 was 23,636 TWh.

Furthermore, the chapter has provided a background on the latest developments of solar photovoltaic technologies worldwide. It recognised the major existing photovoltaic cell/module technologies, such as those manufactured from the crystalline silicon, thin film, organic/polymer, hybrid PV and the dye-sensitized materials.

A comprehensive survey of the existing energy storages that are used for electrical power applications has been presented. The systems are divided into the electrical, mechanical, chemical and the thermal energy storage systems. All these technologies have been carefully surveyed, in relation to their characteristics, storage capabilities, round-trip efficiencies, life spans, and limitations. New storage systems that have relatively lower adverse environmental impacts were also mentioned.

Some related existing research has also been reviewed. It is clear that the majority of the research does not consider solar photovoltaic energy losses, battery state of charge, reliability, future load growth and the life cycle impact analyses, which are very crucial for proposing realistic energy systems for isolated communities. It is these gaps that this thesis intends to address. 


\section{Chapter 3}

\section{Microgrid Planning Strategy}

Energy planners, policymakers and governments have identified renewable energy-based microgrid technologies as an option for electrifying remote communities around the world. However, it is particularly important to have the necessary knowledge and understanding of the appropriate strategies that are required for the successful planning and development of such energy systems [144]. The absence of such approaches can lead to poor, unreliable and unsustainable energy systems, which will potentially affect the proliferation of renewable energy systems. Though the focus of this thesis is not on the implementation of energy systems, the strategies are introduced as general guidelines for understanding and planning off-grid electricity systems in developing countries, including Nigeria.

This chapter provides a planning framework for the proposed microgrid solutions and it is divided into four major parts. The first presents the stages of microgrid system development, while the second part focuses on the component design approach for the solar photovoltaic microgrid (SPM) system. The third section provides the approach for evaluating the economic performance of the proposed microgrid solutions, while the fourth part of the chapter presents the methodology for assessing the environmental impact of the proposed SPM systems. A summary concludes the chapter.

\subsection{Stages of microgrid development}

The diagram shown in Figure 9 presents the phases of the microgrid projects for off-grid applications [144, 145]. These different stages are discussed in practical terms with respect to the necessary strategies required for developing the energy systems. The stages in this section are introduced as the main phases of planning, developing and managing off-grid electricity systems, and are based on the global engineering standards and practical experience [131-143]. 


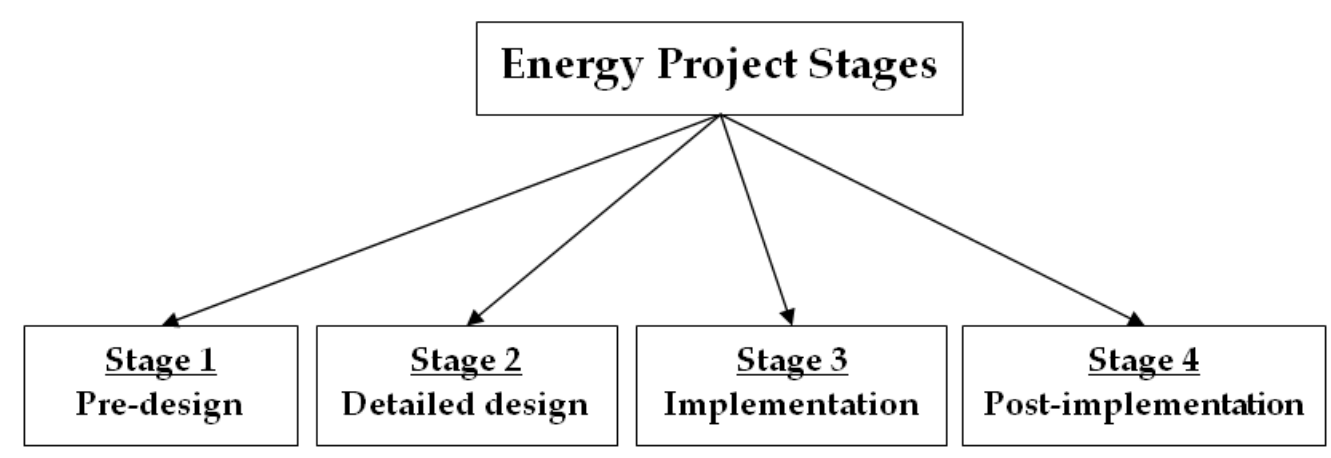

Figure 9: Stages of energy project

\subsubsection{Pre-design stage}

This phase of the electricity planning process concentrates on assessing the specified site where the energy project is to be implemented. It is also known as the preliminary or pre-installation phase [144]. Its main purpose is to provide the energy planner with the required knowledge and understanding of the characteristics of the intended location or community. This includes the geographical and the technical features, as well as the information of the people's status and energy requirements. In essence, this involves considering the location's weather condition and pattern, available energy resources, the types of buildings and distance between them, the intended users' energy needs and consumption patterns and their financial status. In addition, it is important to identify whether or not there is an existing electricity grid around the community, as well as ascertaining the community structure and its accessibility etc.

The seasonal ambient temperature, humidity, rainfall or water flow data is needed to determine some of the location's environmental features that are required for further planning and design. Furthermore, understanding the community's topography is also crucial, i.e. finding out whether it is situated on the hill, in a valley or on a plain terrain etc. This is because it can potentially affect the choice and performance of the proposed energy technologies. These preliminary evaluations are very vital because the information about the site characteristics is one of the major planning requirements.

The electricity planning and development processes are also expected to be in harmony with people's energy needs, i.e. load demand [146]. The users' load demand requirements are best understood by assessing their basic energy needs. Such 
assessments also consider their financial status and ascertains whether or not they are willing to pay for the proposed energy service. It is important to ensure the awareness, participation and the maximum support of the community. This will help to achieve successful projects through awareness programmes and frequent meetings with the stakeholders in the community. Such stakeholders include the community leaders or chiefs or the community development association.

In circumstances where the electricity grid extension is practicable (i.e. technically and economically) in a particular site, the technical procedures for supplying energy to the people will usually be decided by the local utility [144, 146, 147]. This process involves setting up of electric poles and then extending electric lines from the existing distribution substation to the community. The time required for this process differs from one community to another, and it is dependent on the community's distance to the existing electricity network, and the approval of the local utility authority to embark on the grid extension project.

On the other hand, an electricity generation system based on conventional and/or renewable energies is usually recommended for off-grid communities when the grid extension is not viable. Such a recommendation is usually made based on the cost and the communities' distance to the electricity grid [7]. It also involves identifying the possibility of using renewable energy and/or conventional energy resources for electrifying the community. Priority is usually given to the application of renewable energy systems; this is due to their perceived potential for clean electricity generation. In addition, they have a lower system operating and life cycle costs compared to the conventional power generation option. However, in scenarios where an electricity supply from both the grid extension and the renewable energy systems is not feasible, conventional energy systems are recommended for the isolated communities, e.g. diesel/petrol-powered systems.

\subsubsection{Detailed design stage}

The intent of this stage of the planning process is to provide the detailed design and analysis of the proposed electric power generation system. The strategies discussed at this stage primarily involve the assessment of the energy resource(s) and the technical analysis to determine the least-cost energy option(s) with a minimum environmental impact [144-148]. It presents a detailed techno-economic design and evaluation of the proposed energy solutions. As it has been mentioned in the 
preliminary stage, the intended users' basic energy needs and consumption patterns need to be analysed, which are then used for the engineering design.

The community's renewable energy resources are assessed to determine whether or not they can provide a reliable energy supply for the people. This could be achieved by evaluating the historical renewable energy data of the location. Some of the required data include the following:

- Solar PV system: the hourly and average daily irradiance $\left(\mathrm{W} / \mathrm{m}^{2}\right)$ and the ambient temperature $\left({ }^{\circ} \mathrm{C}\right)$

- Wind system: the hourly and average daily wind speed $(\mathrm{m} / \mathrm{s})$

- Small hydro system: the hourly and average water flow $\left(\mathrm{m}^{3} / \mathrm{s}\right)$, and the head $(\mathrm{m})$

Resource assessment is also necessary for predicting the performance of the proposed energy production technologies, in relation to the available data [146]. This way, the energy planner will be able to ascertain which of the available renewable energy resources is viable or most viable for the community.

The technical analysis of the strategy involves evaluating the intended users' demand, component sizing and selection, technology selection, and the system operation and maintenance procedure. This aspect is a very important aspect of the planning process because a poor technical analysis will definitely lead to the system's underperformance or failure. The energy requirements of a particular community are peculiar to the intended applications [147]. The ability to identify these requirements could help to achieve an optimum system design and a desirable performance. The key task is therefore to design an electricity system that will suit the users' energy requirements, and their local community, with the prime goal of achieving high system performance and long-term viability.

Some common household appliances include the light bulbs, electric fans, radios, TVs, DVD players etc, depending on the category of people that is being considered in the design [7]. The users' load demands and usage patterns that have been assessed are then employed to develop the community's load profile. This is used for modelling the appropriate energy systems that can support the users' demand requirements.

Furthermore, the technical analysis is not conducted in isolation; it is combined with the economic evaluation for the purpose of determining cost-effective energy option(s). For this reason, the process is referred to as the techno-economic analysis 
because it integrates both the technical and the economic perspectives. However, the selection of appropriate technology is not only determined by the techno-economic evaluation. It also involves a practical consideration, such as the maturity of the technology, annual availability of the energy resources, the ease with which the proposed system could be operated and maintained, and the availability of spare parts [146]. The energy generation technology could be fueled by either renewable and/or non-renewable energy resources. The renewable energy-based systems are referred to as single-source systems, while the energy production technology based on both the renewable and the non-renewable energy systems is regarded as the hybrid system. In the case of the hybrid energy systems, it is desirable to optimise the renewable energies in their energy mix. This is to encourage the maximum use of renewable energy resources.

\subsubsection{Implementation stage}

While the pre-design and the detailed engineering design stages focus on design and analysis, the implementation and commissioning stage is concerned with the execution of the proposed energy system. The implementation stage brings the technical solutions and plans to reality at the intended community [144]. It involves the approval of the technical design and analysis of the electricity system, obtaining quotations from component manufacturers, deploying equipment and materials to project site, instaling the system, testing the project and listing of the spare parts. At this stage, it is important to ensure that the installation is done with the approved design specifications, according to standards and best practices. This also involves using quality components and materials and providing security for the construction materials on the site.

\subsubsection{Post-implementation stage}

This stage, also known as the post-installation phase, focuses on the tasks, processes and activities after the project implementation. The strategies at this stage involve effective operation and maintenance, system management and monitoring, training and capacity building. In order to achieve a sustainable energy system in the community, it is crucial to have good maintenance measures, such as the preventive and corrective aspects. Experiences have shown that, apart from a poor system design, the lack of maintenance is another major problem that contributes to the failure of 
renewable energy systems [114]. Therefore, maintenance is a crucial aspect at the postimplementation stage that needs to be carefully considered in the energy planning and development parlance.

Furthermore, it is necessary to ensure an effective electric generation and demand management. This is required to maintain the energy system within its rated capacity, which is one of the key ingredients of achieving technical sustainability. In the energy design stage, the system's performance is predicted with the available energy resource, while in the post-implementation stage, the actual system's performance is monitored. The post-installation stage also considers how the electricity system could be scaled up in the future to support the users' energy demand increase.

Capacity building is also another important aspect of the post-implementation stage. This basically involves stakeholders', and particularly the users' participation in the energy project [146]. Local capacity building is usually achieved by providing the technical support and possible training programmes to equip some selected community members with the knowledge and skills on the operation and maintenance of the energy system. This can assist in ensuring the continuous operation of the off-grid electricity system.

This approach has been reported to be effective in some developing countries [147], where people' education and training within the community have paved the way for involving different individuals, such as teachers, traders, technicians, local health workers etc. Also, training and capacity can be organised throughout the energy development phases, which can help to develop local expertise for achieving sustainable community-based energy systems. Another benefit of the training is that it will educate the people about energy efficiency; this will provide the trainees with the necessary knowledge for efficient use of energy.

Some major approaches for planning and developing energy system for remote communities are summarised in Table 1. These strategies are used as part of the background for the methodologies introduced in this thesis, which focus on creating new energy systems for off-grid communities in different parts of Nigeria. They are also discussed because the thesis attempts to carefully consider the long-term viability of the design, right from the inception. Apart from the system design and cost considerations, some other factors affect the planning, development and success of the off-grid electricity systems. 
The lack of good planning is a major factor that contributes to increased solar photovoltaic system failure in Nigeria. Though this thesis majorly concentrates on the first two stages (i.e. pre-design and detailed design) for proposing the solar photovoltaic microgrids for Nigeria, the third and fourth stages (i.e. implementation and post-implementation) are considered for future purposes. The pre-design and the detailed design are an aspect of system planning, while the implementation and the postinstallation are a part of system operation $[7,114]$. The discussion on pre-design and detailed design stages in this thesis are largely based on the author's practical experience and the familiarity with the people, geography and characteristics of the specified communities in Nigeria. The analysis is also guided by the global standards specified by the IEEE and IEC.

Table 1: A summary of general planning approaches [144]

\begin{tabular}{lcl}
\hline Project stage & Approaches \\
\hline Pre-design : & - & Examining the site characteristics \\
& - & Finding out if there is an existing distribution grid \\
& - & Investigating the practicability of extending the grid \\
& to the community if an electricity grid exists \\
& - & Considering grid extension if it is feasible and cost- \\
& effective \\
& - & Considering distributed electric power generation if \\
& grid extension is not practicable in the community \\
& - & Examining the possible energy option(s), e.g. \\
& conventional and/or renewable energy-based \\
& systems \\
- & Understanding people's basic energy needs and \\
& how the project may be funded \\
- & Detailed energy resource assessment \\
- & Energy demand analysis and load profiling \\
- & Techno- economic design and evaluation \\
- & Technology selection \\
& - & Evaluating the system's environmental impact \\
\hline Detailed design: & - & Using quality/approved components and materials \\
- & Instaling components with best practices \\
- & Building local expertise through training \\
- & Providing a list of spare parts \\
- & Providing security during installation \\
\hline Implementation: &
\end{tabular}




\begin{tabular}{ll}
\hline Post-implementation: & - Energy use management \\
& - Effective operation and maintenance \\
& - Considering and managing the future load growth \\
- & Local capacity building \\
\hline
\end{tabular}

\subsection{Component design approach}

Decentralised energy generation systems could be powered by either renewable and/or conventional energy resources. They can be 100\% renewable or conventional energy systems (i.e. single source systems), or be a combination of the two resources (i.e. hybrid systems). As shown in Fig. 3, a solar photovoltaic system, for instance, consists of the PV module, charge regulator, battery storage, inverter, cables etc. The design approaches for the different components of the energy system is discussed in this sub-section, and they are based on the global engineering standards [131-143] and $[6,7]$.

\subsubsection{Load demand model}

The users' energy needs assessment is a crucial step in planning, decision-making and development purposes. It is necessary to have the information on the types of electrical appliances/loads used by the people in a community, the total number of the appliances, their rated power and the daily duration of energy usage. This is then used to analyse the users' daily energy consumption and produce the community's load profile. The community's daily total energy use, in this case, is the summation of the daily energy consumed by the individual users.

- The energy use or consumption could be defined as the quantity of energy that the intended users require per day. It is important to note that the energy demand differs from one person to the other, and this must be carefully considered in order to calculate the total consumption. The daily energy consumption of a particularly community, made up of different users with different loads or appliances could be evaluated by Eq. (9) [148].

$$
E_{u}=\sum_{z}^{\text {category }} A_{z} *\left(\sum_{j}^{\text {load }} n_{j z} \cdot r_{j z} \cdot d_{j z}\right)
$$

where: 
$E_{u}$ is the total energy demand $(\mathrm{kWh})$

$A_{z}$ is the category of users

$n_{\mathrm{j} z}$ is the number of loads

$r_{j z}$ is the rated power of the load within the category

$d_{j z}$ is the duration of operation of load within the category

This thesis, however, focuses on the category of users within the household sector, who are living in off-grid communities. The information on the total energy demand could then be used to develop the load profile.

- The load profile is the users' electric energy consumption pattern as a function of time. It is typically the energy demand over a 24-hr period. It is otherwise regarded as the daily load curve [149], which represents a composite of energy demands by the category of users in the community. The operation of loads varies with time (e.g. daily, weekly, seasonal, etc.), depending on the users' lifestyles. This is evident in the users' status, preferences and availability. A typical load profile is expected to provide information about the users' daily minimum and the maximum load demand, so that the variation of energy usage can be determined. The lowest and the highest values of electrical load demand in a day are regarded as the minimum and the peak demand, respectively, while the load factor is obtained by dividing the average load over a certain period of time by the peak load at that same period [149]. The daily load factor could be estimated by Eq. (10). The energy consumption and the load profile are interwoven. This is because the value of one could be calculated from the other. For instance, the users' total energy consumption may be estimated from a load profile by employing the trapezoidal technique, which basically calculates the area under the load curve, as presented in Eq. $(11)[7,158]$.

$$
\begin{aligned}
& D_{l f}=\frac{L_{a v} \times 24 h r}{L_{p k} \times 24 h r} \\
& E_{u}=\frac{1}{2}\left[L_{i} t_{i}+2\left(\sum_{i}^{m-1} L_{1+i} t_{1+i}\right)+L_{n} t_{n}\right]
\end{aligned}
$$

where:

$D_{\text {lf }}$ is the load factor (\%)

Lav is the average load $(\mathrm{kW})$

$L_{p k}$ is the peak load $(\mathrm{kW})$ 
$L_{i}$ is the electrical load at time $t_{i}(\mathrm{~kW})$

$L_{n}$ is the electrical load at time $t_{n}(\mathrm{~kW})$

The users' energy needs assessment is vital for designing and modelling the energy system. This involves evaluating the daily energy consumption and developing the load curve. Furthermore, this thesis considers the seasonal variations of the users' energy demand. In this case, the users' energy consumption is examined for two seasons, (i.e. dry and rainy seasons), which is informed by the lifestyles of the category of people considered. During the dry season, there is a high solar irradiance, and all the specified users' appliances may be supported by the proposed PV system. However, during the rainy season, the PV system may need to be over-sized to be able to support the users' appliances. This is due to relatively lower irradiation cycle during the rainy season [4-8]. Alternatively, the users' load demand could be reduced in proportion to the available solar irradiance during the rainy season. These aspects are carefully considered in this study, to propose practical energy production models for the specified isolated households.

- Load growth is an important aspect of the energy system design and planning for remote communities that must also be carefully considered. In this thesis, it is first considered in terms of the effect of increasing the number of households on the proposed energy solutions. It is then further expressed as $25-75 \%$ load demand increase of the baseline value of $175 \mathrm{kWh} / \mathrm{d}$. The load growth factor, $l_{g f}$ is introduced into the PV sizing model to determine the additional capacities that can help the proposed solar photovoltaic microgrids to withstand the users' increased demand.

\subsubsection{Energy resource assessment}

The assessment of the available energy resource(s) is also crucial in order to provide technical solutions for the proposed community's load profile. This aspect involves evaluating the renewable energy resources in the community. In this thesis, solar energy is selected as a possible means of electrifying the off-grid communities in Nigeria. The country experiences abundant solar energy resource, which is around an average of $\sim 4$ to $6.5 \mathrm{kWh} / \mathrm{m}^{2}$ per day, with the northern part of the country having a higher potential compared to the southern part $[4-8,150]$. The peak sun hours $(P S H)$ of the different locations in the country are determined from this information. 
The thesis uses an hourly solar irradiance and temperature data to model the proposed SPM systems for six different communities, one from each of the country's six geopolitical zones. Such information serves as an input to the simulation software to determine the appropriate SPM capacities and also to evaluate the systems' performance.

\subsubsection{Photovoltaic array sizing}

The performance of solar photovoltaic modules or arrays is dependent on two major factors, such as the solar irradiance and temperature parameters of the specified community $[6,7,18,67,151-153]$. The literature establishes that the current produced by a solar PV array increases with the solar irradiance. However, as the ambient temperature increases (i.e. beyond $25{ }^{\circ} \mathrm{C}$ ), the array power output and the efficiency are decreased. The reduction of the power output and the efficiency is due to a decrease of the array voltage, which has been adversely affected by an increase in the cell temperature. As a result, it is important to carefully consider the losses due to the temperature in any PV-based electricity systems.

For c-Si photovoltaic cells, the open circuit voltage $\left(V_{o c}\right)$ decreases by about $0.37 \%$ per ${ }^{\circ} \mathrm{C}$ increase in temperature, while the short circuit current increases only by around $0.05 \%$, under the same operating condition [18]. The end result of the increase of temperatures of the solar PV modules is that the maximum power point (MPP) shifts away to the left, as shown in Fig. 7. This leads to a reduction in their maximum power outputs, by around $0.5 \%$ per ${ }^{\circ} \mathrm{C}$ rise in temperature. The information about the temperature coefficient of power for c-Si PV modules differs from one manufacturer to another, but most of them are $\leq 0.5 \%$ per ${ }^{\circ} \mathrm{C}[13,18,19,56,69,151]$. Furthermore, for c-Si solar modules whose temperature coefficient of power is not provided by the manufacturer, a value of $0.5 \%$ per ${ }^{\circ} \mathrm{C}$ is used for analysing their electrical performance. Because the currents of solar photovoltaic modules are less affected by the temperature variations compared to their voltages, the temperature dependence of the short-circuit current is in most cases neglected.

This thesis emphasises the temperature dependence of the module voltage, power output, energy yield and losses and the efficiency. The temperature dependence of the solar PV module open-circuit voltage is represented by Eq. (12); the temperature dependence of the module efficiency is estimated by Eq. (13), while the PV module's actual temperature is calculated by Eq. (14) [56, 69, 152]: 


$$
\begin{gathered}
\mathrm{V}_{o c, \text { new }}=V_{\mathrm{oc}, S T C}\left(1-\left(K_{v}\right)\left(T_{c}-T_{\mathrm{c}, S T C}\right)\right) \\
\eta_{\text {new }}=\eta_{S T C}+\left[1+K_{p}\left(T_{c}-T_{c, S T C}\right)\right] \\
T_{c}=T_{a}+\left(\frac{N O C T-20^{\circ} C}{0.8}\right) G_{a}
\end{gathered}
$$

where:

$V_{\text {oc,new }}$ is new open-circuit voltage (V)

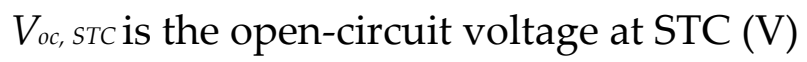

$K_{v}$ is the temperature coefficient of voltage $\left(/{ }^{\circ} \mathrm{C}\right)$

$K_{p}$ is the temperature coefficient of power $\left(/{ }^{\circ} \mathrm{C}\right)$

$T_{c}$ is the cell temperature $\left({ }^{\circ} \mathrm{C}\right)$

$T_{c, \text { STC }}$ is temperature at STC $\left({ }^{\circ} \mathrm{C}\right)$

$\eta_{\text {new }}$ is the new efficiency (\%)

$\eta_{\text {STC }}$ is the efficiency at STC (\%)

$T_{a}$ is the ambient temperature $\left({ }^{\circ} \mathrm{C}\right)$

NOCT is the nominal operating cell temperature $\left({ }^{\circ} \mathrm{C}\right)$

$\mathrm{G}_{a}$ is the solar irradiance of the location $\left(\mathrm{kW} / \mathrm{m}^{2}\right)$

The IEC 61836 standards specify that three factors are responsible for losses in solar PV systems. These are the temperature effect, the incomplete utilisation of solar irradiation and the balance of system (BOS) losses. In this thesis, the losses due to the incomplete utilisation of solar irradiation and the BOS losses are referred to the "other losses". Therefore, apart from the losses due to temperature, the other losses are due to the following [143]:

- inverter losses

- dust (i.e. soiling of the module)

- shading

- wiring and conversion

- ageing etc.

The possible losses due to temperature could be evaluated the design stage, using the location's solar irradiance and temperature data. However, to assess the other losses, a 
scaling factor is usually introduced that considers the reduction in the power output of PV modules assuming the real-life operating conditions, compared to the STC conditions provided by the manufacturer. Such a factor provides considerations for the other losses in practical terms. It is referred to as the derating factor and is being introduced to assess the solar module power output, according to [136, 152-155].

This thesis is also based on the worst-case scenario, i.e. the users' highest load demand and the lowest monthly average of solar irradiation of the location, according to the IEEE 1562 standard. Based on this, the ideal SPM's capacity can be calculated by Eq. (15), but the actual or the baseline capacity is obtained by introducing the design safety factor $\left(d_{s f}\right)$ [158]. This factor is used to multiply Eq. (15) to determine the suitable SPM's capacity in any location of interest. The solar PV array is first oversized by $20 \%$ to obtain an initial basis (i.e. baseline). In this case, the design safety factor $\left(d_{s f}\right)$ is 1.2 and the solar array will be analysed to ascertain whether or not, it will be sufficient to support the users' demand requirements over the year. The power output of the solar photovoltaic microgrid, $P_{o}$, at a particular hour of the day or year is therefore estimated by Eq. (16) [56, 69, 136, 152]:

$$
\begin{gathered}
P_{b}=\frac{E_{u}}{P S H} \\
P_{o}=\frac{G_{a}}{G_{S T C}}\left[x \cdot P_{m p} \cdot \mathrm{d}_{r f}\left(1+K_{p}\left(\mathrm{~T}_{c}-T_{c, S T C}\right)\right)\right]
\end{gathered}
$$

where:

$P_{b}$ is the PV capacity $(\mathrm{kW})$

PSH is the monthly average peak sun hours of the location $x$ is the number of PV modules $P_{m p}$ is the rated power of the module $(\mathrm{kW})$

The IEC 61724 standard provides recommendations on the photovoltaic system performance monitoring- guidelines for measurement, data exchange and analysis. Its recommendations are used to guide the performance analysis reported in this thesis. The IEC 61724 standard makes the following considerations [7, 155]:

- There is a disparity in the electrical performance of solar PV systems around the world, due to the difference in rated system power, locations, seasons, 
engineering design and analysis, and the module technology employed. It is, therefore, a challenging task to compare PV modules or arrays from different regions. The standard has, however, introduced certain performance parameters that could be used as a basis for comparing various solar photovoltaic modules. Examples of such parameters are normalised yields, losses and performance ratio

- The performance parameters are expected to provide insights into the deficiencies in system operation, as well as the system losses. Solar PV system losses include losses due to temperature, incomplete utilisation of the solar irradiation and the inefficiencies or failure of components

- The performance parameters would be able to validate solar PV performance evaluation models at the detailed design stage of the planning process

\subsubsection{Charge regulator sizing}

Solar charge regulators are sized by considering the PV array current and voltage, and the battery system voltage. This is because their voltage values must be matched with those of the solar photovoltaic array and battery storage systems. In addition, it is essential that they are able to handle the maximum current that is generated by the solar PV array. These factors guide the selection of the charge regulators. Eq. (17) [131, 141] can be used to estimate the size of charge regulators. In solar photovoltaic systems, there are two widely recognised types of charge regulators; these are the pulse width modulation (PWM) and maximum power point tracker (MPPT) types. The MPPT type is preferred by designers and installers because of its relatively higher capability to maximise the capture of the solar energy resources [139]. The charge regulated is overrated by $30 \%$ as presented in Eq. (17) so that it can accommodate a surge current from the solar PV array.

$$
C R_{\text {size }}=1.3 \times I_{s c}
$$

where:

$C R_{\text {size }}$ is the capacity of the charge controller

$I_{s c}$ is the short-circuit current $(\mathrm{A})$ 


\subsubsection{Battery model and sizing}

The Kinetic Battery Model (KiBaM) is used in this study to explain the behaviour of the lead-acid battery system. It involves determining the quantity of electrical energy that is stored by or discharged from the battery storage bank each "time step" [152]. The $\mathrm{KiBaM}$ is fundamentally based on the theory of "electrochemical kinetics" that models battery energy storage banks as a double tank system, where the "available energy" and the "bound energy" are contained in the first and the second tank, respectively. The available energy represents the energy that is readily available for conversion to DC electricity, while the bound energy is the energy that is chemically bound and is not directly available for withdrawal. The two-tank concept is described by the following parameters, which are determined from the battery's capacity curve:

- The theoretical or maximum battery capacity $\left(Q_{\max }\right)$ - the total quantity of energy that can be contained by the double tanks

- The capacity ratio (c) - the ratio of the size of the first tank to the combined size of both tanks. The first tank is the one that contains the available energy

- The rate constant $(\mathrm{k})$ - the rate at which the battery can convert the bound energy to the available energy or vice-versa. This is associated with the conductance between the two tanks

The total amount of energy that is stored in the battery at any particular period of time is obtained by the sum of the available and the bound energy, as presented by Eq. (18):

$$
Q_{t}=Q_{a e}+Q_{b e}
$$

The maximum amount of power that the battery bank can discharge for a particular length of time $\Delta t$ is given by Eq. (19). The maximum amount of power, which the battery bank can store over a particular time, could similarly be estimated by Eq. (20).

$$
\begin{aligned}
P_{d} & =\eta_{d} P_{d K \text { KKaM }} \\
P_{c} & =\eta_{c} P_{c \text { cKiBaM }} \\
P_{d K i B a M} & =\frac{-k c Q_{\max }+k Q_{a e} e^{-k \Delta t}+Q k c\left(1-e^{-k \Delta t}\right)}{1-e^{-k \Delta t}+c\left(k \Delta t-1+e^{-k \Delta t}\right)}
\end{aligned}
$$




$$
P_{c K i B M}=\frac{k Q_{a e} e^{-k \Delta t}+Q k c\left(1-e^{-k \Delta t}\right)}{1-e^{-k \Delta t}+c\left(k \Delta t-1+e^{-k \Delta t}\right)}
$$

Eqs. (19) and (20) represent the allowable range for the power into or out of the battery bank, in any one time step. The power into the battery is considered to be positive, while the power out of it is negative. Two additional constraints are imposed on the battery bank's charge power, which relate to both the battery's maximum charge rate $(M C R)$ and the maximum charge current $(M C C)$. The values of the battery charge power that correspond to the MCR and MCC can be estimated by Eqs. (23) and (24)

$$
\begin{aligned}
P_{C M C R} & =\frac{\left(1-e^{-\alpha_{c} \Delta t}\right)\left(\mathrm{Q}_{\max }-\mathrm{Q}\right)}{\Delta t} \\
P_{C M C C} & =\frac{N I_{M C C} V_{s y s}}{1000}
\end{aligned}
$$

where:

$P_{d}$ is the max. amount of power that battery can discharge over time $(\mathrm{kW})$

$P_{c}$ is max. amount of power that can be stored by the double tank $(\mathrm{kW})$

$P_{\text {дКівам }}$ is the KiBaM value of maximum power that can be discharged $(\mathrm{kW})$

$P_{\text {сківам }}$ is KiBaM value of maximum power that can be stored $(\mathrm{kW})$

$P$ is power into or out of the battery bank $(\mathrm{kW})$

$p$. $f$ is power factor of the system

$Q_{a e}$ is available energy at the beginning of the time step (kWh)

$Q_{b e}$ is the bound energy at the beginning of the time step (kWh)

$Q$ is total energy in the battery at the beginning of the time step ( $\mathrm{kWh}$ )

$Q_{\max }$ is the total capacity of the battery bank (kWh)

$Q_{a e E N D}$ is bound energy at the end of the time step ( $\mathrm{kWh}$ )

$Q_{b e E N D}$ is available energy at the end of the time step $(\mathrm{kWh})$

$\Delta t$ is the length of the time step (h)

$\alpha_{c}$ is the maximum charge rate of the battery (A/Ah)

$\eta_{c}$ is the battery charge efficiency

$\eta_{d}$ is the battery discharge efficiency

$c$ is the battery capacity ratio

$i$ is the hourly interval 
$n$ is the number of hours in a day

The maximum battery charge power is then obtained at a value corresponding to the minimum value of the three parameters in Eq. (25), with the assumption that each of them occurs after the charging losses are considered. Similarly, the battery discharge losses are assumed to exist after the energy is drawn from the double tank. In this analysis, both the charge and the discharge efficiencies are obtained from the square root of the round trip efficiency, thus, they are equal. The round trip efficiency of leadacid batteries is usually around $80 \%$ [152].

$$
P_{c}=\frac{\operatorname{MIN}\left(P_{c K i B a M}, P_{c M C R}, P_{c M C C}\right)}{\eta_{c}}
$$

After obtaining the values for the actual charge or discharge power, then, the resulting values of $Q_{a e}$ and $Q_{b e}$ at the end of the time step can be calculated by Eqs. (26) and (27):

$$
\begin{aligned}
& Q_{a e E N D}=Q_{a e} e^{-k \Delta t}+\frac{(Q k c-P)\left(1-e^{-k \Delta t}\right)}{k}+\frac{P c\left(k \Delta t-1+e^{-k \Delta t}\right)}{k} \\
& Q_{b e E N D}=Q_{b e} e^{-k \Delta t}+Q(1-c)\left(1-e^{-k \Delta t}\right)+\frac{P(1-c)\left(k \Delta t-1+e^{-k \Delta t}\right)}{k}
\end{aligned}
$$

The battery bank's size is determined through Eq. (28) [7, 131-143, 156, 157]. Also, the availability of the PV microgrids is investigated on hourly and monthly basis over the year. This is achieved through the battery state of charge $(S o C)$ analysis. The battery SoC relates the available microgrids' outputs to the communities' maximum load demands. When the energy produced by the PV microgrids are more than the users' load demands, there is a positive energy difference, which is referred to as excess energy. However, there is a negative energy difference when the microgrids' outputs are less than the users' demands, and this is called an energy deficit. The energy balance is defined by Eq. (29) [158].

$$
\begin{gathered}
B_{c p}(\text { req.capacity })=\frac{E_{u} \times D_{a}}{\eta_{r t p} D o D V_{s y s}} \\
E_{d i f f}=\sum_{i=1}^{8760}\left(E_{G E N}-E_{u}\right)_{i}
\end{gathered}
$$

where: 
$B_{c p}$ (req. capacity) is the battery capacity (Ah)

$D_{a}$ is the autonomy

$\eta_{r t p}$ is the round-trip efficiency (\%)

$D o D$ is the depth of discharge (\%)

$V_{\text {sys }}$ is the system voltage $(\mathrm{V})$

$E_{u}$ is the total energy demand $(\mathrm{kWh})$

$E_{\text {diff }}$ is the energy balance

$E_{G E N}$ is the hourly energy generation $(\mathrm{kWh})$

Battery storage systems are designed to store the excess energy so as to forestall an energy deficit; this energy is used when the sun is not available $[6,14,169]$. The energy deficit is the inability of the solar photovoltaic microgrids to support the energy demand at a particular time $[158,159]$. Such an occurrence is quantified in terms of the unmet energy (Unmetenergy) or unmet load demand. Therefore, the ratio of the total unmet energy to the total demand, over a period of time (e.g. an hour, 24-hour, month, year) is what is being regarded as the loss of energy probability ( $L O E P)$ as presented in Eq. (30) [158, 159]. Hybrid Optimisation Model for Electric Renewables (HOMER) simulation tool is used to analyse the battery energy, unmet load demand, LOEP and the availability indices.

$$
L O E P=\frac{\sum_{i=1}^{8760}\left(\text { Unmet }_{\text {energy }}\right)_{i}}{\sum_{i=1}^{8760}\left(E_{u}\right)_{i}}
$$

\subsubsection{Battery charge and discharge constraints}

The electrical energy stored by the battery system is bound by the following constraints $[152,158,168]$ :

$$
B_{c}(\min ) \leq B_{c} \leq B_{c}(\max )
$$

where:

$B_{c}$ is the battery capacity (Ah)

$B_{c}(\max )$ is the product of the rated battery capacity $(\mathrm{Ah})$ and the rated system voltage; this is the specification provided by the manufacturer.

$B_{c}(\min )$ is the minimum battery capacity $(\mathrm{Ah})$ 
SoC is the state of charge at a particular hour, $i$

$$
\begin{aligned}
& B_{c}(\min )=(1-M D O D) * B_{c}=\left(S o C_{\min }\right) * B_{c} \\
& S o C(i)=\frac{B_{c}}{B_{c}(\max )}
\end{aligned}
$$

Eq. (31) is also be translated to Eq. (34) by relating the battery capacity to the battery state of charge. The loss of energy $(L O E)$ is expected to occur if the battery $S o C<S o C_{\text {min, }}$ otherwise it is zero. In this thesis, the $S_{o} C_{\min }$ is $30 \%$, while the $S_{o} C_{\max }$ is $100 \%$.

$$
S o C_{\min } \leq S o C \leq S o C_{\max }
$$

Also, the relationship between the battery's expected life span $\left(N_{\text {years }}\right)$ and its cycle life $\left(N_{\text {cycles }}\right)$ is given Eq. (35) [2]:

$$
N_{\text {years }}=\frac{N_{\text {cycles }}}{365}
$$

The following are the parameters that affect the battery energy storage system's performance, according to Eq. (28):

- Days of autonomy: this represents the time during which the solar photovoltaic system is capable of supporting the load in the absence of sunlight. It needs to be carefully considered at the system's design stage, as it affects the technoeconomic evaluation of the proposed solutions. The higher the autonomy, the larger the size of the battery. Therefore, autonomy is dependent on the consideration of the cost, location and the system type. For instance, a location with a low solar irradiance will have a higher autonomy, while the one with a high solar irradiance will have a relatively lower autonomy

- System voltage: this represents the voltage of the battery bank. The lower the system voltage, the higher the battery capacity in Ampere-hours. However, the higher the system voltage, the lower the battery capacity. Experience has shown that battery banks that are designed with multiple strings of monoblock batteries (e.g. 6V, 12V etc) $[156,157]$ usually experience a poor system performance and reduced life spans. This is due to unequal charge and discharge rates between the several battery strings. Such arrangements are also associated with a relatively higher maintenance requirements and costs. High-capacity battery 
systems (i.e. those $\geq 50 \mathrm{kWh}$ capacity) are used in practice to address this problem. This allows limiting the strings to a maximum of 1 to 3 series-connected 2 V cells, instead of monoblocks [157]

- Demand: it can be concluded from Eq. (28) that the larger the load demand, the larger the required battery capacity that would support it. This also has an economic implication. For this reason, the use of energy efficient appliances will help to reduce the burden of the load demand on the energy system

\subsubsection{Inverter sizing}

The output of PV systems is direct current (DC), and most of the users' appliances operate on the alternating current (AC) supply. The inverter is employed to change the DC to AC output for operating the users' loads. The sizing methodology for the inverter depends on the types of loads that are used, such as the resistive and inductive loads. Eq. (36) is used to estimate the inverter's power rating.

$$
\operatorname{In} v_{\text {size }}=3 \times\left(L_{\text {ind }}\right)+L_{o}
$$

where:

$I n v_{\text {size }}$ is the inverter capacity $(\mathrm{kVA})$

Lind is the inductive load $(\mathrm{kW})$

$L_{o}$ is the other loads $(\mathrm{kW})$

As seen in Eq. (36), the power rating of the inductive loads is multiplied by a factor of 3. This is due to their relatively higher current requirements when they are switched on (i.e. surge currents at starting). It is necessary to carefully consider this so that the loads will not place too harsh an effect on the inverter system. In other words, the inverter size must be large enough to support the users' loads at any given time (i.e. it should not be lower than the total load). The inverter voltage must also be matched with the battery voltage. A poorly designed inverter system will invariably lead to PV system's failure, given that the system is off-grid.

It is also possible to have an inverter system that incorporates solar charge controllers with maximum power point tracking (MPPT) capability. Such a device is selected in this thesis. This is because it has a better capacity to maximise the solar energy than the pulse-width modulation (PWM) type [139]. 


\subsubsection{Low-voltage network model}

The proposed microgrid systems are designed to supply electricity to a number of households within small communities. The system design considers the community layout, i.e. the arrangement of the houses and their distances to the power plant. The energy supply system is typically a low-voltage network, i.e. $400 \mathrm{~V}, 50 \mathrm{~Hz}$ system. A simulation tool is required to calculate the load flow. This shows the relationship between the energy produced by the system and the users' load demand. A lot of tools are in existence for load flow calculations, but in this thesis, the DIgital SImuLation of Electrical NeTworks (DIgSILENT) PowerFactory software is used. This software is a computer aided engineering tool for analysing electric power systems.

Load flow calculations are used to examine electrical power systems under the steady-state operating conditions [163]. The steady-state conditions could be defined as the operating conditions whereby the variables and the parameters are assumed to remain unchanged for a particular period when the system is being examined. Apart from estimating the electrical energy generated with respect to the load, the software also allows the evaluation of the busbar voltages [149].

The proposed solar photovoltaic microgrid system is modelled in the DIgSILENT environment and it consists of the PV array, the battery and the single-phase loads. The photovoltaic array and the battery components are obtained from the software's template folders. These components are configured as static generators, and as the name suggests, they are non-rotating generators such as the following $[7,163]$ :

- photovoltaic system

- fuel cells

- storage devices

- HVDC terminals

- reactive power compensators

Configuring the PV and the battery components as static generators implies that they are capable of delivering AC power outputs through the static converters that are embedded in their models. 
Furthermore, it is important to select suitable conductors or lines that will be used to supply electricity to the houses. Such conductors are selected from the global library of DIgSILENT. Apart from this, it is also important to select the right phase technology. A 3-phase with neutral technology is appropriate for LV systems, which is employed to distribute energy to the single-phase loads in the different houses. A single-phase technology is common in Nigeria's residential dwellings. In such supply arrangements, it is common to distribute the users' loads on the 3-phase system, usually with a conductor size of $4 \times 16 \mathrm{~mm}^{2}$. The selected lines have a maximum operational temperature of $70^{\circ} \mathrm{C}$, which resembles a real life (i.e. outdoor) operating condition. The busbars in the model are labelled as LV_1 to LV_k, (i.e. for k-bus power generating system), while the lines connecting the busbars and the loads in the houses are represented by $\mathrm{L} 1$ to $\mathrm{Ln}$, (i.e. for $\mathrm{n}$ lines).

The distances between the power generating system and the houses are also considered in the model, which are obtained from the community's layout. This provides information about the required length of conductors or lines. The electrical system is divided into branches according to the arrangement of the houses and the distribution of the single phase models on the $400 \mathrm{~V}$ 3-phase microgrid system. Running a series of load flow calculations with 3-phase loads in DIgSILENT involves the use of AC load flow with balanced 3-phase calculation method. However, AC load flow with unbalanced 3-phase method is usually employed for single-phase load models. This also involves devising a means of balancing the system voltage. The acceptable steady state voltage value is between 0.95 p.u and 1.05p.u [7, 163, 168].

\subsubsection{Diesel generator fuel consumption model}

Engine generators (e.g. diesel generators) are usually designed to support the peak electrical load requirements of any particular application. The system alternator must be capable of supplying a continuous apparent power (kVA) of the load [142]. The primary reason for this is that engine generators are most efficient when they operate at a higher proportion of their rated power [7, 140, 166]. However, they become less efficient as the loads are decreased. The operation of the generator between 50 and $80 \%$ of its rated power is recommended by IEC [142]. 
When the loads are decreased, diesel generators usually have a relatively higher specific fuel consumption (litre per $\mathrm{kWh}$ ) [166]. The generator's fuel consumption rate could be estimated by Eq. (37) $[8,152]$. The diesel generator may be the only power source for electrifying a particular location. It may also be used as back-up with renewable energy in certain periods in the year when there may be a loss of energy. However, this thesis focuses on $100 \%$ solar PV systems, whose techno-economic and life cycle environmental performance analyses will be compared to that of a diesel power generation system.

$$
F_{c}=X P_{\text {out }}+Y P_{\text {rated }}
$$

where:

$F_{c}$ is the fuel consumption rate (litre per $\mathrm{kWh}$ )

$P_{\text {out }}$ is the operating output power $(\mathrm{kW})$

$P_{\text {rated }}$ is the rated generator power $(\mathrm{kW})$

$\mathrm{X}$ is the generator fuel curve slope (typically 0.246 litre/kWh) [152]

$Y$ is the fuel curve intercept coefficient (typically 0.08415 litre/kWh) [152]

\subsection{Economic performance}

A life cycle cost analysis ( $L C C A$ ) technique is used to evaluate the proposed microgrid system's costs [152]. The LCCA is conducted for different energy scenarios involving different system component sizes. The thesis then compares the proposed system's economic performances with that of a diesel power system.

The life cycle cost $(L C C)$ of a system is the sum of all the costs incurred during its lifetime. The LCC is also referred to as the total net present cost (NPC) [152]. It is indisputable that the cost of the components cannot be the same over the system's life span. Therefore, comparing similar components or items at different times and costs is an easier method to reduce all the costs to purchasing time [167]. This is popularly known as the present value method and it suggests that two major factors usually determine the value of money over a period of time. These are the inflation and the interest rates. 
- Inflation rate: this measures the degree to which the value of money has declined. The inflation rates of $4 \%$ and $5 \%$ have been assumed by the authors in [119] and [190], respectively, but a value of 5\% is assumed in this thesis [158]

- Interest rate: this provides the information about the amount of profit that is obtainable from saving a sum of money. The interest rates of $8 \%$ and $7 \%$ have been assumed by the authors in [119] and [190], respectively, but a value of $6 \%$ is assumed in this thesis. An interest rate of $6 \%$ and a lifetime of 25 years are recommended in the Hybrid Optimisation Model for Electric Renewables (HOMER) software - a commercially available microgrid design and planning tool developed by the National Renewable Energy Laboratory (NREL), for the purpose of analysis [152]

The inflation and interest rates in Nigeria change with time, which is why in energy project planning, development and management, all costs and economic evaluations are usually reviewed and updated when the bill of engineering quantities (BoEQ) is being prepared [217]. The BoEQ is a document that provides the list of materials, components, and labor, and their current costs required for the implementation of a proposed energy project.

As mentioned earlier in Chapter 3, there are four stages of microgrid or rural electrification development life cycle, such as the pre-design, detailed design, installation and post-installation. There are a lot of processes between the detailed design and the installation stages. One of these is the review and re-evaluation of costs. In practice, this is carried out by a quantity surveyor or a cost consultant. It is important to note that all the costs - initial component, installation, operation and maintenance and replacement costs will be considered based on the current market price before the procurement of materials and components, mobilisation and installation take place. All other costs such as the community levy or community development association levy, local authority processing charges etc., will also need to be considered. 
It is not usually known at the design stage, when an energy project will eventually be implemented. This, and the fluctuations in the investment rates justify the fact that any interest and inflation rates used for the initial economic analysis are subject to change when the project is about to commence in the future [217, 218]. The historical averages of the country's inflation and interest rates may also be assumed, but these will be modified in the future. By using assumed inflation and interest rates of 5 and $6 \%$, respectively, this thesis presents an economic analysis of the proposed SPM systems that can be a reference point for researchers and energy developers and managers. The analysis could be adjusted or modified to suit the actual situation in the future.

Costs may be expressed as "current costs" and "constant costs" [219]. The actual cash flows obtained in the market are the current costs. The current costs change over time as a result of inflation (or deflation). The constant cash flows represent the costs that would have been required if they were paid in the base year. The base year refers to the year that the evaluation is structured around, and it is safe to assume a constant inflation rate during the project lifetime [152, 219]. Therefore, this thesis assumes a constant inflation rate of $5 \%$, indicating that all costs are assumed to rise at the same rate. Furthermore, time value of money is the price attached to the time that investors wait for a return on investments [219]. The interest rate is a measure of this time and it is crucial to the calculation of present value.

The inflation and the interest rates, and the number of years involved are required to determine the present value coefficient $(P V C)$. The present value coefficient of money is given by Eq. (38), which represents the PVC of a component that will be purchased in n-year later. The component's present value could, therefore, be estimated by Eq. (39). Furthermore, other costs are usually involved over the project's lifetime, and such costs are referred to as the recurring expenses in particular years. These costs are primarily the operation and maintenance $(\mathrm{O}$ and $\mathrm{M})$, and the replacement costs. They can be estimated by Eq. (40) by introducing each year in which the component has been purchased and/or maintained. 
The proposed microgrid system's life cycle costs could be estimated by Eq. (41) [7, 166-169]. The initial capital cost basically includes the cost of the solar PV modules, battery bank, charge regulator, inverter, and other BOS - cables, circuit breakers etc. The installation cost is also a part of the initial capital cost. The system's annual life cycle cost $(A L C C)$ is usually estimated by multiplying the capital recovery factor $(C R F)$ and the LCC as presented in Eqs. (42) and (43). The cost per kWh of energy over the project's lifetime is calculated by Eq. (44) $[7,158,166]$. In addition, the cost parameters are presented in Tables 2 and 3.

$$
\begin{gathered}
P V C=\left(\frac{1+i}{1+r}\right)^{n} \\
P_{v}=P V C \times C_{i c} \\
P V C=C_{i c}+C_{i c}\left(\frac{1+i}{1+r}\right)+C_{i c}\left(\frac{1+i}{1+r}\right)^{2}+C_{i c}\left(\frac{1+i}{1+r}\right)^{3}+\ldots . .+C_{i c}\left(\frac{1+i}{1+r}\right)^{n-1} \\
C_{L C C}=C_{i c}+\mathrm{C}_{o m}+\mathrm{C}_{r p} \\
C R F=\frac{\left((1+r)^{n} \times r\right)}{\left((1+r)^{n}-1\right)} \\
A L C C=C R F \times L C C \\
C O E=\frac{A L C C}{E_{u}}
\end{gathered}
$$

where:

$i$ is the inflate rate (\%)

$r$ is the interest rate (\%)

$n$ is number of years

$C_{i c}$ is the initial capital cost (\$)

$C R F$ is the capital recovery factor

$P V C$ is the present value coefficient

$P_{v}$ is the component's present value (\$)

$A L C C$ is the annual life cycle cost (\$)

$C_{L C C}$ is the life cycle cost (\$) 


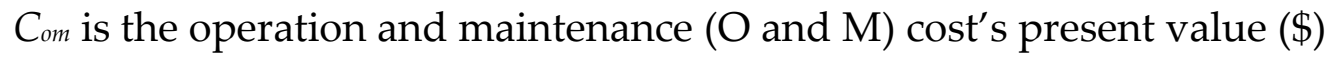

$C_{r p}$ is the replacement cost's present value (\$)

$C O E$ is the cost of energy $(\$ / \mathrm{kWh})$

$E_{u}$ is the useful electrical energy that is produced by the energy systems

There are other financial metrics like the return on investment and payback time, and the funding mechanism for energy projects. These are not discussed in this thesis but they will be considered in future studies.

Table 2: SPM's cost parameters

\begin{tabular}{ll}
\hline Parameter & Value \\
\hline Inflation rate (\%) & 5 \\
Interest rate (\%) & 6 \\
Number of years & $1,2,3, \ldots$. etc \\
Project lifespan (years) & 25 \\
Module cost (\$/W) & $1.15[220]$ \\
Battery cost (\$/cell) & $191.6[192]$ \\
Inverter cost (\$) & $23,350[193]$ \\
Capacitor $(\$)$ & $76.2[221]$ \\
$4 \times 16 m{ }^{2}$ cable (\$/m) & $1.81[223]$ \\
Installation cost (\$) & $5 \%$ of components cost \\
O and M cost $\$$ ) & $2 \%$ of installation cost \\
PV lifespan (years) & 25 \\
Battery lifespan (years) & $5[192]$ \\
Inverter lifespan (years) & $10[193]$ \\
Capacitor lifespan (years) & 10 \\
Length of cable (m) & 532.375 \\
No. of battery cells & 348 \\
No. of inverter & 1 \\
No. of capacitor & 1 \\
No. of replacement for PV & 0 \\
No. of replacement for battery & 4 \\
No. of replacement for inverter & 2 \\
&
\end{tabular}


No. of replacement for capacitor 2

Table 3: Diesel power system's cost parameters

\begin{tabular}{ll}
\hline Parameter & Value \\
\hline Inflation rate $(\%)$ & 5 \\
Interest rate $(\%)$ & 6 \\
Number of years & $1,2,3, \ldots .$. etc \\
Project life (years) & 25 \\
Generator cost $(\$)$ & 12,189 for a $15 \mathrm{kVA}$ rating [222] \\
Installation cost $(\$)$ & $5 \%$ of components cost \\
O and M cost $(\$ / \mathrm{yr})$ & 250 \\
Shipping cost $(\$)$ & $500[222]$ \\
Generator lifespan (years) & 4 [222] \\
No. of generators & 2 \\
No. of replacement for generator & 5 \\
Fuel cost $(\$ /$ liter $)$ & $0.85[5]$ \\
Cost of cable $(\$ / \mathrm{m})$ & $1.81[223]$ \\
Length of cable $(\mathrm{m})$ & 532.375 \\
\hline
\end{tabular}

\subsection{Environmental performance}

The proposed solar photovoltaic microgrid systems' environmental performances will be examined in the following ways:

- analysing the amount of fuel saved

- evaluating the emissions minimised

- assessing the emission rate and the global warming potential (GWP)

- estimating the cumulative energy demand (CED)

- calculating the energy payback time (EPBT)

- estimating the energy return on investment (EROI) 
The methodology specified in [152, 158, 213] is employed for estimating the amount of fossil fuel saved by the energy systems. However, the value of the amount of $\mathrm{CO}_{2} / \mathrm{kg}$ of diesel, i.e. emission factor, is obtained from the literature to estimate the emissions that could be avoided by the proposed systems, compared to a scenario of $100 \%$ diesel power generation. The amount of fuel saved by using $100 \%$ solar photovoltaic system, in this case, would be assumed to be the amount of fuel consumed by $100 \%$ diesel power system [152].

\subsubsection{Global warming potential}

In the majority of the existing related research, the emissions avoided by $100 \%$ solar PV system are assumed to be equal to the emissions produced by $100 \%$ diesel generator over the project's life. In such studies, the emissions by the energy systems are not considered from the point of view of the life cycle impact. Therefore, this thesis carefully considers the analysis of the proposed systems' life cycle emissions. This can provide better insights into the systems' environmental impact. The GWP provides information about the greenhouse gases (GHGs) emitted during the SPM systems' lifetimes $[129,158]$. It is usually quantified in $\mathrm{gCO}_{2}$-eq or $\mathrm{kg} \mathrm{gCO}_{2}$-eq.

One of the advantages of the solar photovoltaic generation technology is that it has a very low carbon footprint compared to the conventional fossil-fueled systems. It also has a very low or no GHGs during operation, but its life cycle is associated with emissions during its manufacturing (i.e. direct emissions) plus the emissions that are embedded in the materials and infrastructure (i.e. indirect emissions) [130]. Most of the PV modules that are used in Nigeria are imported from other countries, most especially from Europe, China, the United States etc. Even the only existing 7.5 MW Solar Panel Manufacturing Plant in Abuja, Nigeria imports its solar cells from other countries [158, 213]. Currently, there is no existing research on solar PV life cycle impact analysis in the country. For this reason, the emission rate of the proposed SPMs is based on the results published in the literature for some of the countries from which Nigeria imports solar PV modules. The values reported in [129], [130] and [198] for the crystalline silicon photovoltaic systems are used in this study.

The carbon footprint is evaluated as the equivalent quantity of $\mathrm{CO}_{2}$ that has the same GWP measured over an integrated timeframe of 100 years [129, 130, 158, 213]. The most recent GWP factors that are reported by the Intergovernmental Panel on Climate Change (IPCC) are the GWP values of 1, 25, 298 and 4,750-14,400 for $\mathrm{CO}_{2}, \mathrm{CH}_{4}, \mathrm{~N}_{2} \mathrm{O}$ 
and chlorofluorocarbons, respectively. These values are used in the literature for determining the life cycle GHGs. The carbon footprint is thus calculated as follows [130]:

$$
C F=\frac{\sum_{j \in G H G} \mu_{j} \cdot C E_{j}}{E_{a G E N}}
$$

where:

$C F$ is the life cycle carbon footprint of the SPM

$j$ is the index of species of emissions that belong to the GHG family

$\mu_{j}$ is the GWP factor that corresponds to the species of emissions $j$

$C E_{j}$ is the "cumulative emissions" of the species of emissions $j$ during the life cycle of the SPM (i.e. both direct and indirect)

$E_{a G E N}$ is the annual electricity production by the SPM

\subsubsection{Cumulative energy demand}

The emission rate and the GWP parameters are used to evaluate the emissionrelated part of the life cycle analysis, while the cumulative energy demand (CED) is employed for assessing the energy-related aspect. Life cycle analysis method is a rigorous exercise that involves evaluating the materials and the energy flow through the solar PV modules' manufacturing, operation and the end-of-life (i.e. cradle-to-grave analysis) [173, 158]. Therefore, the CED expresses the energy requirements over the solar photovoltaic system's life cycle. The energy requirements are usually valued as a primary energy. The primary energy and the cumulative energy demand are therefore defined as follows [130, 158, 173, 198]:

- Primary energy - is the energy embodied in natural resources, which has not gone through any anthropogenic conversion and needs to be transformed and transported to become energy that is fit for use.

- Cumulative energy demand - refers to the total energy demand that is valued as primary energy over the solar photovoltaic system's life cycle. Basically, it includes both the direct and the indirect energy consumption and is calculated by Eq. 46.

$$
C E D=E_{\text {ma }}+E_{t r}+E_{i n}+E_{o p}+E_{d e}
$$


where:

$E_{m a}$ is the primary energy requirements for manufacturing

$E_{t r}$ is the primary energy requirements for transport

$E_{\text {in }}$ is the primary energy requirements for installation

$E_{o p}$ is the primary energy requirements for operation

$E_{d e}$ represent the primary energy requirements for decommissioning.

$E_{m a}$ is also referred to as the embodied energy of manufacture and is responsible for over 90\% of the PV system's life cycle carbon footprints [158, 173, 198, 213].

\subsubsection{Energy payback time}

For each year the proposed SPM systems are in operation, they are expected to displace some amount of energy that would have been required of the conventional energy systems - diesel power plants. The EPBT is another most frequently used solar PV environmental performance metric, which represents the time required to compensate for the total primary energy (i.e. both renewable and non-renewable energies) requirements over the SPM systems' lifetimes [129, 130, 158, 173, 198, 212, 213]. It is calculated by Eq. (47):

$$
E P B T=\frac{C E D}{E_{a G E N} / \eta_{g}}
$$

where:

$\eta_{g}$ is the coefficient for converting primary to electrical energy (\%)

$E_{a G E N}$ is the yearly energy production $(\mathrm{kWh})$.

\subsubsection{Energy return on investment}

This parameter measures the quantity of electricity that can be returned for the investment per unit of primary energy $[130,158]$. In other words, the EROI of the SPM is the ratio of how many times over its life span it would generate the cumulative energy requirements for its manufacture [61]. This parameter is also referred to as the net energy ratio (NER) [38] or energy yield ratio (EYR) [66]. It is dimensionless and is calculated by Eq. (48).

$$
E R O I=\frac{S P M_{l i}}{E P B T}=\frac{S P M_{l i} \cdot E_{a G E N}}{\eta_{g} \cdot C E D}
$$


where:

$S P M_{l i}$ is the solar photovoltaic microgrid's lifetime (i.e. 25 years in this study).

An EROI $>1$ indicates that the energy production over the SPM system's operational life is larger than the energy that is initially invested in the manufacturing process of its PV modules [129, 130, 158]. This means that there is a higher net energy

production by the solar photovoltaic system. However, an ERO1 < 1 indicates that the energy system is not a renewable technology since its primary energy requirement is more than the electricity it produces over the life cycle.

\subsection{Chapter summary}

This chapter has presented the approaches for planning the proposed SPM systems. It has discussed in detail the stages of microgrid system planning and development processes, such as the pre-design, detailed design, implementation and post-implementation stages. Though microgrid implementation is not the focus of the thesis, the presented strategies serve as general guidelines for designing and planning energy systems for off-grid applications in developing countries. Such strategies can be useful for achieving long-term viability of off-grid electric power systems.

The chapter has also discussed detailed approaches for sizing the major components of the SPM systems. These include the load demand modelling, energy resource assessment, solar photovoltaic array sizing, the inverter/controller sizing, and the battery capacity estimation. Also in this process, the design safety factor technique is proposed for determining the SPM systems' capacities. This is achieved through a detailed solar photovoltaic performance evaluation, based on the relationship of the required design parameters. These parameters include the users' highest daily demand, peak sun hours and the solar irradiance of the location, solar irradiance at STC, rated PV array maximum power at STC, derating factor, the temperature coefficient of power of the PV array, PV array temperature and the array temperature at STC. Sizing the SPM systems by this technique is expected to provide a better means of determining different solar PV capacities for a particular community.

Furthermore, approaches for determining the energy losses, battery SoC, and the unmet energy, loss of energy probability, availability, and the load growth have been carefully described. The low-voltage SPM network model was presented, which is designed according to the community's layout. Thus, it supplies energy to 24 different houses, using six different locations in Nigeria as case studies. The diesel generator's 
fuel consumption rate model has also been presented for estimating the quantity of fossil fuel that would be consumed, supposing a diesel power plant is used to electrify the specified communities.

The methodologies for assessing the life cycle cost (LCC) and the life cycle impact (LCI) have also been discussed, which are used for estimating the proposed system's economic and environmental performances. The chapter has also presented the approaches for estimating the emission rate, GWP, CED, EPBT and the EROI. In the next chapter, six different locations in Nigeria will be used as case studies for the proposed SPMs. 


\section{Chapter 4}

\section{Microgrid systems for Nigeria}

This chapter uses selected small communities from Nigeria's six geo-political zones as case studies for the proposed solar photovoltaic systems. The chapter is divided into six main parts. The first presents a brief information about Nigeria; this includes the geographical characteristics such as the coordinates, location, land mass, climate and seasons, geo-political zones and the solar energy resources. The second part discusses the country's energy situation, while the third section provides a background of the selected communities. The fourth section presents the communities' load demand and profile. The fifth part discusses the communities' peak sun hours and the ambient temperatures. The sixth section presents the solar photovoltaic microgrid model based on the information presented in the third, fourth and the fifth sections. A summary concludes the chapter.

\subsection{Brief information about Nigeria}

Nigeria is a country situated in West Africa with a total area of $923,768 \mathrm{~km}^{2}$ (i.e. 356,667 sq. miles) and its population is given as 182.2 million people $[6,170]$. Out of the country's total area, only $1.4 \%$ is water. This means that it has a land mass of $98.6 \%$ of the total area. It lies within the latitudes $4.32^{\circ} \mathrm{N}$ and $14^{\circ} \mathrm{N}$ and longitude $2.72^{\circ} \mathrm{E}$ and $14.64^{\circ} \mathrm{E}$, sharing borders with Cameroon eastward, Republic of Benin and Niger westward and northward, respectively, and the Atlantic Ocean southward. It is the $7^{\text {th }}$ most populous country in the world, preceded by China, India, United States of America, Indonesia, Brazil, and Pakistan that takes the $1^{\text {st }}$ to $6^{\text {th }}$ position, respectively [170].

The country is divided into six geo-political zones, constituting a total of 36 states and the Federal Capital Territory (FCT), in Abuja [165]. In addition, the country's landscape, the physical and the vegetation features differ from a flat grassland (i.e. open savannah) in the northern region to the thick rain forests (i.e. plantation) in the southern region. This is coupled with several lakes, rivers and mountainous locations in different parts of the country. The country's six geo-political zones include the North-Central (NC), North-East (NE), North-West (NW), South-East (SE), South-South (SS) and the 
South-West (SW) [144]. These zones are divided into states (see Tables 4 and 5). In addition, there are 774 local government areas (LGAs) in the 36 states of the country.

The country possesses a tropical climate of two major seasons - the dry and the rainy seasons. The features of these seasons differ in the country's northern and southern parts. The northern region basically experiences the short rainy and the long dry seasons. The ambient temperatures during the day are constantly high (up to about $\left.40^{\circ} \mathrm{C}\right)[7,171]$. This is usually associated with a relatively low humidity all-year-round and with only a little or no cloud cover. The short rainy season is usually between July and September, while the long dry season is between October and June.

On the other hand, the southern region experiences four different seasons, such as the long rainy, short dry, short rainy and the long dry seasons. The long rainy season occurs between March and July, with the peak occurring in June and noticeable because of thick clouds. The short dry season usually occurs over about three to four weeks in August, with its peak around the last two weeks of the month. The short rainy season usually occurs from the beginning of September to the middle of October, with its peak occurring towards the end of September. The short rainy period is not as heavy as that of the long rainy period. The short dry season between the two rainy seasons is the time to harvest and plant grains, e.g. corn [171]. The long dry season occurs around the end of October till the beginning of March, with its peak usually occurring between early December and the end of February. This period is referred to as Harmattan, where vegetation growth is impeded and leaves fall off from trees as a result of low moisture content in the air.

The country has vast deposits of crude oil, natural gas and other natural/mineral resources (e.g. gold, copper, timber, solar, hydro etc). However, this thesis concentrates on the solar energy aspect of the country's resources. The country has solar energy resource, averaging about 4 to $6.5 \mathrm{kWh} / \mathrm{m}^{2}$ per day (i.e. average total of about 1,460 to $2,373 \mathrm{kWh} / \mathrm{m}^{2}$ per year). The northern part of the country has higher potential compared to its southern counterpart [4-8, 150], as shown in Figure 10. This solar energy potential makes photovoltaic systems a suitable option for electricity generation. 
Table 4. States in the northern region

\begin{tabular}{lll}
\hline Geo-political zones & State & State Capital \\
\hline North-Central & Benue & Makurdi \\
& Kogi & Lokoja \\
& Kwara & Ilorin \\
& Nasarawa & Keffi \\
& Niger & Niger \\
& Plateau & Jos \\
Forth-East & Federal Capital Territory & Abuja \\
& Adamawa & Yola \\
& Bauchi & Bauchi \\
& Borno & Maiduguri \\
& Gombe & Gombe \\
& Taraba & Jalingo \\
& Yobe & Damaturu \\
North-West & Jigawa & Dutse \\
& Kaduna & Kaduna \\
& Kano & Kano \\
& Katsina & Katsina \\
& Kebbi & Birnin Kebbi \\
& Sokoto & Sokoto \\
& Zamfara & Gusau \\
\hline
\end{tabular}

Table 5. States in the southern region

\begin{tabular}{lll}
\hline Geo-political zones & State & State Capital \\
\hline South-East & Abia & Umuahia \\
& Anambra & Awka \\
& Ebonyi & Abakaliki \\
& Enugu & Enugu \\
Imo & Owerri \\
South-South & Akwa Ibom & Uyo \\
& Cross River & Calabar \\
& Bayelsa & Yenogoa \\
Rivers State & Port Harcourt \\
& Delta & Asaba \\
& Edo & Benin City
\end{tabular}




\begin{tabular}{lll} 
South-West & Ekiti & Ado Ekiti \\
Lagos & Ikeja \\
Ogun & Abeokuta \\
Ondo & Akure \\
Osun & Osogbo \\
Oyo & Ibadan \\
\hline
\end{tabular}

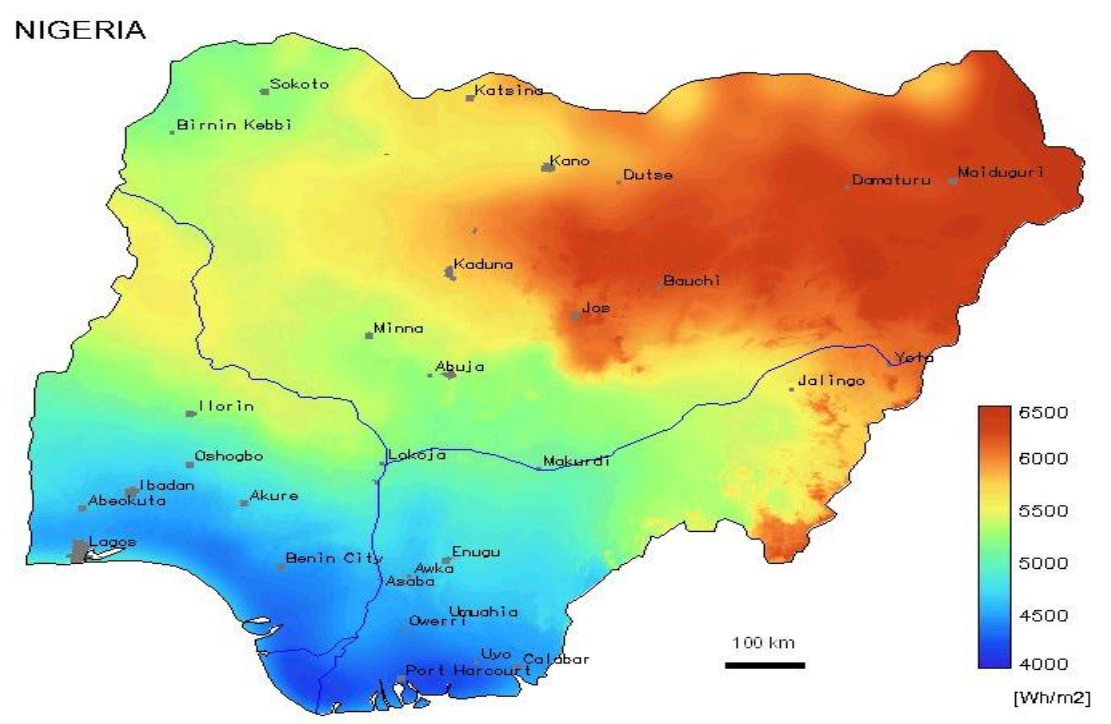

Figure 10: Solar energy resources across the geo-political zones of Nigeria [150]

\subsection{Energy situation of the country}

The country's available electricity generation capacity is less than 5,000MW [4, 5]. Also, its national, urban and rural electrification rates are 45\%, 55\%, and 35\%, respectively, according to the recent IEA World Energy Outlook report [3]. Nigeria's electricity access deficit rate is about 60\%; therefore, the greater proportion of its population does not have access to electricity[4-8].

About $50 \%$ of the urban and $70 \%$ of the rural population do not have access to the power grid. This further reveals that the majority of the villages and rural communities in the country are not electrified. Nigeria's centralised power infrastructure is currently fueled majorly by two energy sources, i.e. gas/oil and water resources. About 79\% of electricity is produced by the thermal plants, while the remaining $21 \%$ of electricity is generated by hydropower plants. Also, out of about $66 \%$ of the electricity generated by 
the thermal plant is fueled by natural gas; the remaining portion of about $33 \%$ is being produced from oil [109].

Electricity is usually produced at the generation plants at around $14-16 \mathrm{kV}$, which is stepped up to $330 \mathrm{kV}$ and then transmitted over long distances. This $330 \mathrm{kV}$ transmission voltage is stepped down to $132 \mathrm{kV}$, and then to primary and secondary distribution voltage levels of $33 / 11 \mathrm{kV}$ and $415 / 220 \mathrm{~V}$, respectively. The electricity supply from the grid is often not reliable, because of the wide gap that exists between the people's load demands and the available power generation capacity (i.e. generation $<$ load demand). Another factor that is responsible for the poor reliability is the use of old and inefficient electrical equipment.

Sadly, there is no clear difference between several on-grid and the off-grid areas in the country. This is because there are a lot of communities in the country that are connected to the grid and do not have electricity supply for 5 years. This poor energy situation has led to increased use of fossil-fueled generators for meeting part of daily electricity requirements in most parts of the country $[4,5,7]$. This development not only increases air pollution in the local community, but also has an adverse effect on the people's health. The emissions released by the generators also contribute to climate change, in addition to the flare gas that the country emits in its oil exploration systems. This situation is prevalent and it needs urgent attention, in order to save the environment. Although the country has lower carbon emissions compared to the industrialised nations, it is necessary for it to participate in the global measures for ensuring environmental sustainability. One of the measures to mitigate the climate change is by increasing the use of renewable energy resources.

This thesis, motivated by the above-mentioned challenges, proposes new electricity systems (i.e. SPM systems) for off-grid communities in different parts of the country. It focuses on how to harness the country's solar energy resources. The outputs of the study can be used for planning and developing new energy systems for remote communities in the country, as well as other developing countries. 


\subsection{Background of selected communities}

\subsubsection{The communities}

The proposed SPMs in this thesis are based on six off-communities, one from each of the country's geo-political zones. The communities, presented in Table 6, have been selected to explore the possibility of using solar photovoltaic systems in different parts of the country. With the communities in focus, the thesis attempts to ascertain the important indices required for planning and developing new electricity systems for different areas.

Table 6: List of selected communities

\begin{tabular}{cccc}
\hline No. & Geo-political zone & State & Community \\
\hline 1. & North Central & FCT & Gwagwalada \\
2. & North East & Bauchi & Bununu \\
3. & North West & Zamfara & Gusau \\
4. & South East & Enugu & Nbulu-Njodo \\
5. & South South & Bayelsa & Amurukeri \\
6. & South West & Ogun & Lisa \\
\hline
\end{tabular}

\subsubsection{The structure}

The study targets low- to middle- income earners, such as teachers, traders, and other civil servants etc., who go to work in the morning and come back home in the evening time [7]. These people live in the outskirts and travel to their different places of work on a daily basis. It is therefore expected that the people's energy consumption will be more in the morning when they are preparing to set out for work, and in the evening when they are back from work. All these are considered to determine the users' load profile, so as to model suitable SPM capacities that can match their requirements.

Energy supply is one of the major problems that these people face. The bigger community is usually made up of smaller on-grid and off-grid communities. The efforts 
made by most of the off-grid communities to get access to the grid, in most cases, have proved abortive [7]. Also, the on-grid communities are usually not different from the off-grid communities, because of the erratic and unreliable grid energy supply. This situation leaves most people in such communities with the petrol/diesel-powered generation option, as a means for meeting a part of their daily energy requirements. This thesis proposes new energy systems for such communities, as a way to minimise the carbon-intensive generating systems with which the people are currently familiar, and also improve the users' lifestyles. Such systems have the potential to increase the country's electricity access rate in the future through the interconnection of clusters of localised microgrids and their integration with the national grid.

Most of the existing solar photovoltaic systems in the country for domestic and/or office applications are designed to supply electricity for only a limited number of hours per day [7], e.g. 5 to 10 hours. The system design and the number of hours of energy supply mainly depend on the intended users' load demands. These are usually restricted by the system cost. For instance, street lighting systems are typically designed for a 12-hour daily energy supply, i.e. a dusk-to-dawn energy requirement.

In addition, the design of water pumping systems based on solar PV also depends on the users' requirements. The users' demand, in this case, is the quantity of water required. However, they are designed for $\sim 2$ to 4 hours operation per day for residential applications. In many homes, fridges are not used due to the poor energy situation and the limited generators' sizes. These generators can only power a few appliances at a time, such as lighting bulbs, fans, TVs. It is against this backdrop that this thesis proposes energy systems that could provide a 24-hour electricity supply for the users' basic energy requirements, including the fridges. Such electricity generation systems will improve the people's lifestyles, while also minimising the use of fossilfueled power systems. The typical electrical appliances used by most of the people living in the specified communities are presented in Table 7 [158].

As presented in Chapter 3, the number of hours of operation of the users' electrical loads will determine the total communities' energy consumption and load 
profile. This is also associated with the users' availability. This thesis does not assume typical rural dwellers or peasants as the intended energy users, but considers those described above. Most are not available in the afternoon, except for weekends and in some homes where the children are around after the school time. Based on this lifestyle, the users' energy consumption in the morning and evening times is expected to be higher than the one obtained in the afternoon. This analysis is used for developing the communities' aggregate load profile.

Table 7: Estimate of typical domestic appliances in the communities

\begin{tabular}{cll}
\hline S/No. & Appliances & Rating (W) \\
\hline 1. & Compact fluorescent lamp (CFL) & $16-20$ \\
2. & Fan & $60-80$ \\
3. & Radio & 40 \\
4. & TVs & $60-150$ \\
5. & DVD player & 35 \\
6. & Fridge & 250 \\
7. & Phone charger & 6 \\
\hline
\end{tabular}

\subsubsection{Assumptions made}

This thesis examines an energy model for supplying a community of 24 households. The model is considered for each of the communities selected in Table 4, as a way to exploring the possibility of new energy systems for different off-grid areas. The following characteristics are assumed:

- The same category of people is considered for the selected communities

- The same aggregate load profile is used for the communities because of the similar lifestyles of the specified category of people across the six geo-political zones. The worst-case community's load demand is considered for the energy analysis 
- The occupants in a single household range from 4 to 6 people

Using these assumptions, the thesis will simulate different energy scenarios and then evaluate the amount of electricity that is likely to be generated in the selected communities, considering the locations' different environmental factors - solar irradiance and ambient temperature.

\subsubsection{Challenges}

There are three major challenges faced by the author in the process of conducting this research. These are:

- No load demand data for Nigeria

- Scarcity of hourly solar irradiance and ambient temperature for Nigeria

- No existing solar PV microgrid model

These problems led to critical thinking on how to advance the solar PV systems research in the country, while addressing the issue of system failure mentioned in Chapter 3, and also filling the identified gaps mentioned in Chapter 2. The author visited several energy-poor communities across the country's six geo-political under the platform provided by the National Agency for Science and Engineering Infrastructure (NASENI) - Nigeria's only Agency instituted by law to create science and engineering infrastructure in the country. The author has also gained experience in energy demand in buildings and electrical installation. These provide the opportunity to be able to identify the common electrical appliances used by different categories of energy users.

The appliances that are commonly used by the category of people considered in this thesis is presented in Table 7. Computers and printers are not included because the majority of them have their desktops in the offices or shops. In addition, electric cookers are not included because they use kerosene stoves. Electric irons are not also included in Table 7; this is not because the users do not have, but most of them take their clothes to a laundry shop where they pay to iron them, while some of the users make use of kerosene stoves or a charcoal iron.

In this case where the users' load data is not available, designing a load profile depends on the competence of the solar PV system designer and the understanding of the intended energy users' lifestyles. As mentioned earlier, fridges are not used in 
several homes in the country. This is also not because they do not have fridges, but due to a lack of energy supply to operate them. The $1-1.5 \mathrm{kVA}$ generators that are commonly used in most houses cannot power the lighting bulbs, TVs, radios, DVD players and fridges together. Therefore, fridges are not operated in such houses. This is the situation in Nigeria, which is why visiting the sites to assess the users' situation, needs, type and the rating of the appliances is necessary for constructing a working load profile.

In doing so, it is important that the load profile for each of the 24 houses conform to the users' lifestyle, while the time that the appliances are operated and the amount of energy consumption vary from one house to another. Visiting different remote houses provided the author an opportunity to ascertain the common appliances used by the intended energy users, as shown in Table 7. This makes a practical sense than making assumptions about the appliances that are likely to be found in the houses without visiting the site [153, 172, 208]. Furthermore, ascertaining the type of appliances used by the people is instrumental to the techno-economic design's load profiling [194].

The problem of the dearth of hourly solar irradiance and the ambient temperature was addressed by obtaining data set from the Solar Energy Services for Professionals, namely the solar radiation data (SoDa) [150]. This data makes the 8760-hr analysis possible in this thesis.

Since there is no existing PV microgrid model and the users do not currently have access to the national grid, this thesis designs new electrification systems that can provide a 24-hr energy supply to the users. The appliances are operated at different times of the day, but the fridges are operated for 24 hours in a day. The SPM systems are designed based on the architectural layout provided by a building consortium in Nigeria [225]. This consortium is a group of registered engineers and planners and has designed the layout for local housing development in the near future. The building consortium's plan is to partner with investors and project developers to build the 24 houses for low- to -medium income earners. Designing a solar photovoltaic system for the houses will be useful for developmental purposes in the future, since energy is one of the important ingredients to make their living in the community comfortable. 


\subsection{Load demand}

\subsubsection{Users' load profile}

The households' estimated load demand profiles are shown in Figures 11(a) to 11(h). The community's load profile is presented in Figure 12 and this is a composite of energy consumption of all the energy demand by the 24 households. The total daily community's energy demand is $174.98 \mathrm{kWh}$, with peak load, average demand and a load factor of about $14.2 \mathrm{~kW}, 7.3 \mathrm{kWh}$, and $51 \%$, respectively. Figure 12 shows that the energy consumption increases in the morning, between 5 and $7 \mathrm{a} . \mathrm{m}$. This is because the users prepare for their daily work at such periods, leading to an increase in the number of appliances (e.g. lighting, radios, TVs etc.) that are operated.

The fridges are operated as base loads and are not switched off from the supply. The energy consumption drops around 8a.m. because most leave for work, therefore, the energy usage decreases. The energy use in the afternoon is relatively lower, though children in some houses may operate the TVs and/or DVD players during that period. The energy demand increases in the evening, from about 5p.m. and it peaks around 8 p.m. due to the increase in the number of appliances that are operated as the users return from work. The demand in the night is relatively higher than the demand during the day because of the operation of electric fans in the houses. This is because the locations considered are part of the tropical regions, and it is a usual practice to operate fans throughout the night.

Table 8 presents the values of the household energy demands. It can be seen that the houses 1 and 18 have the lowest and the highest daily demands of about $5.70 \mathrm{kWh}$ and $9.74 \mathrm{kWh}$, respectively. 


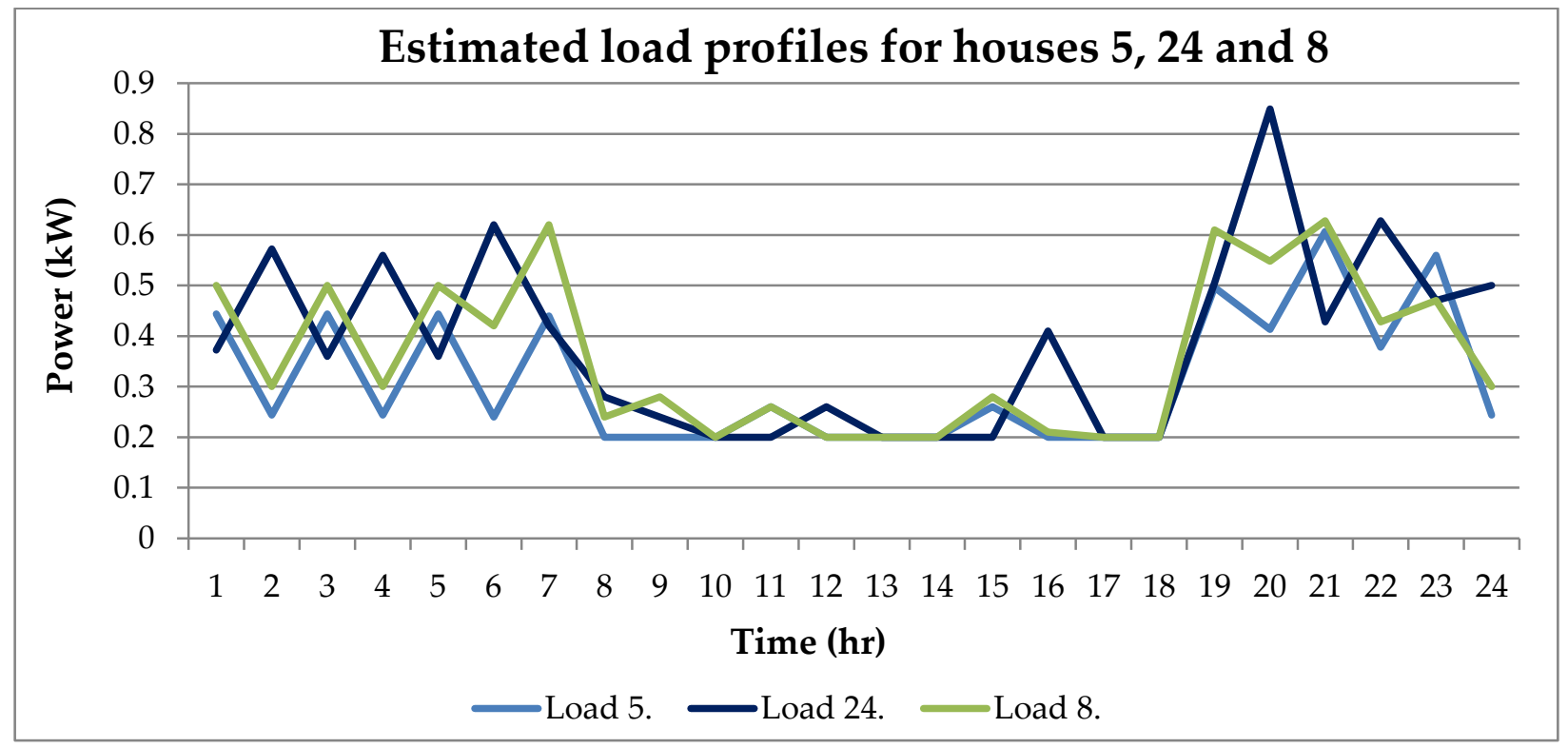

(a)

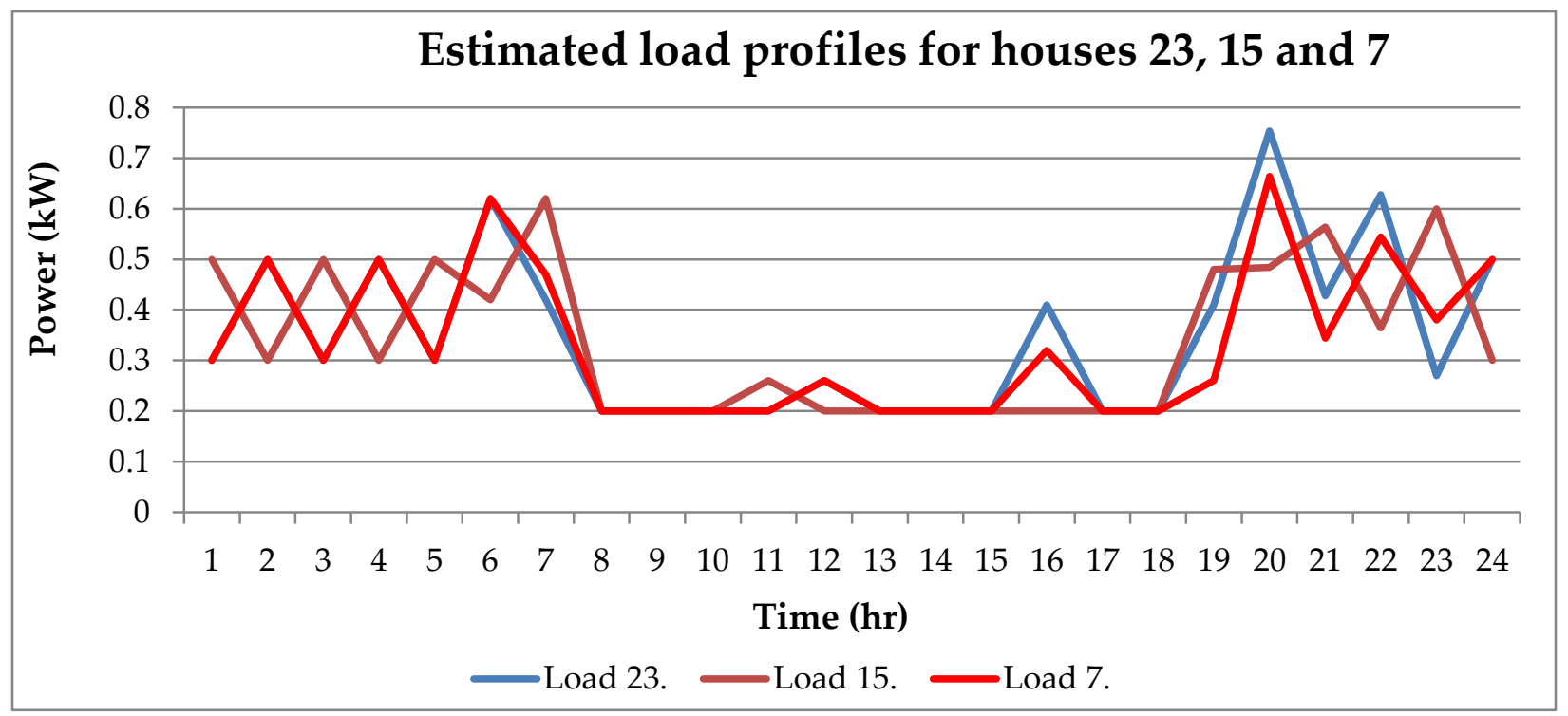

(b) 


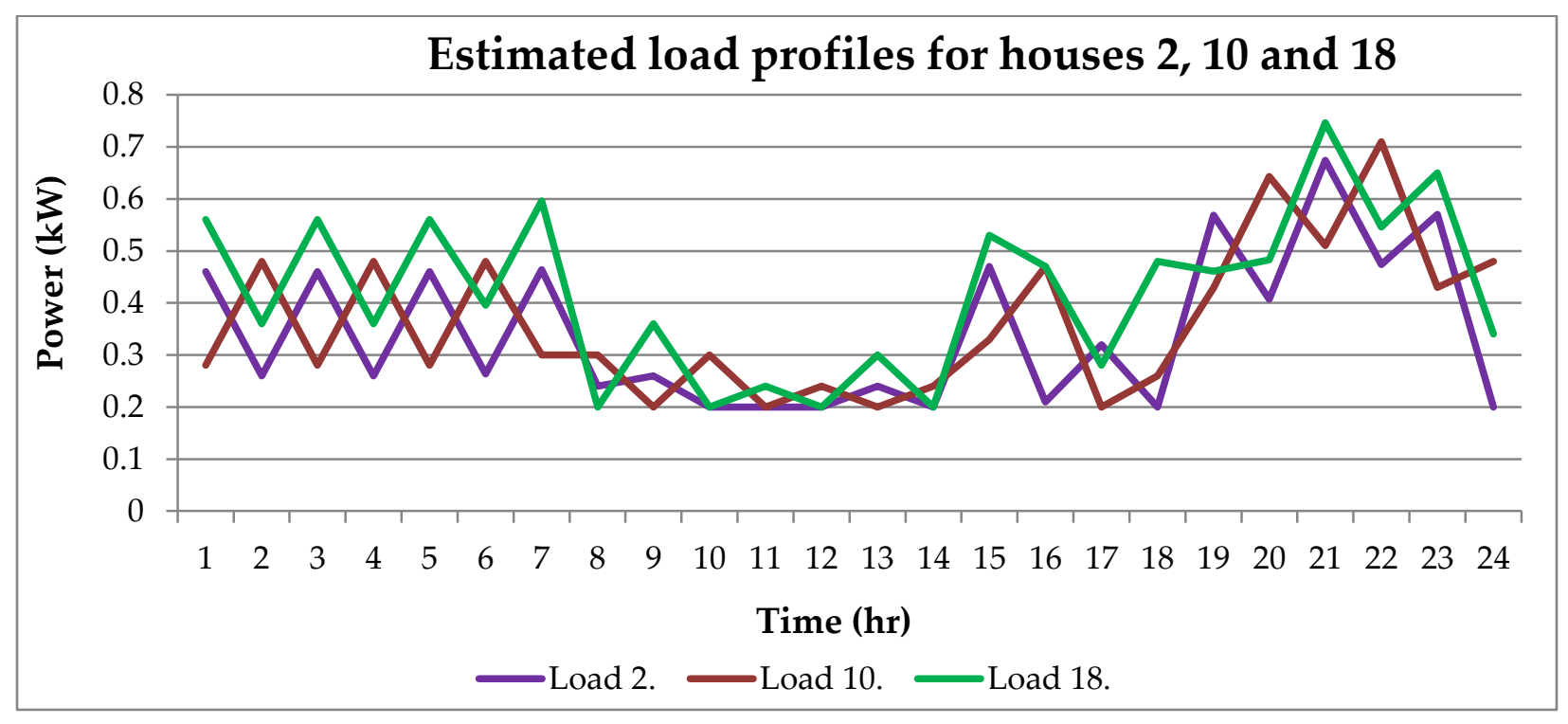

(c)

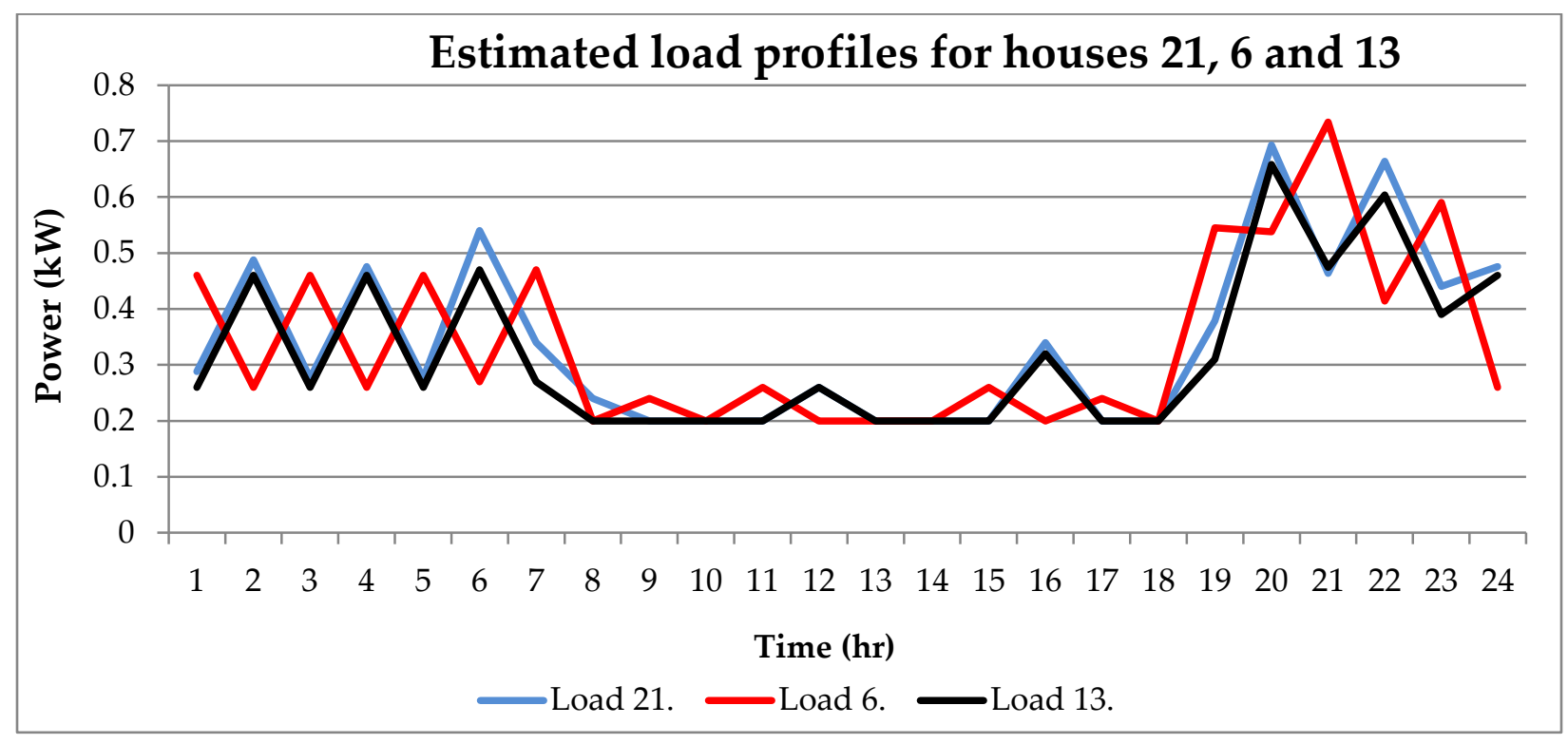

(d) 


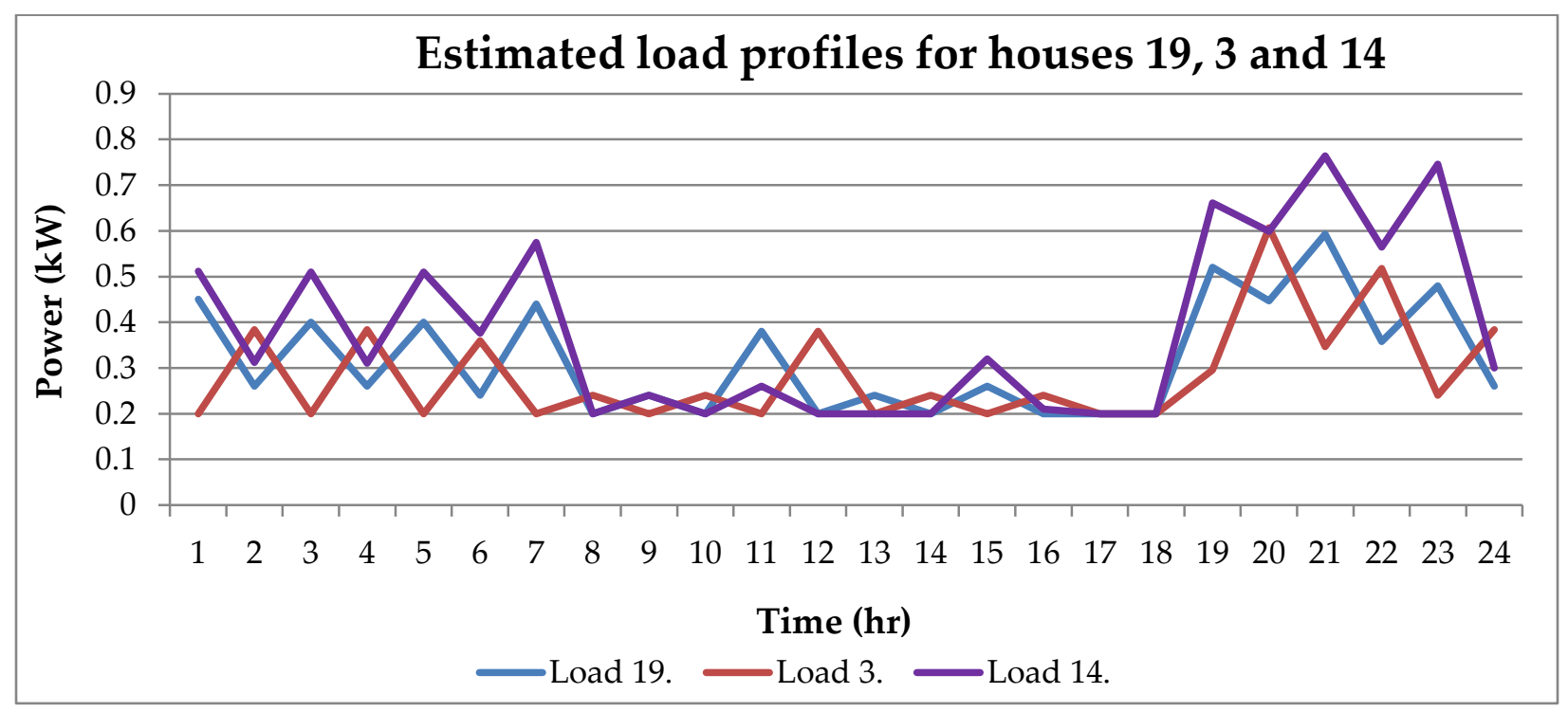

(e)

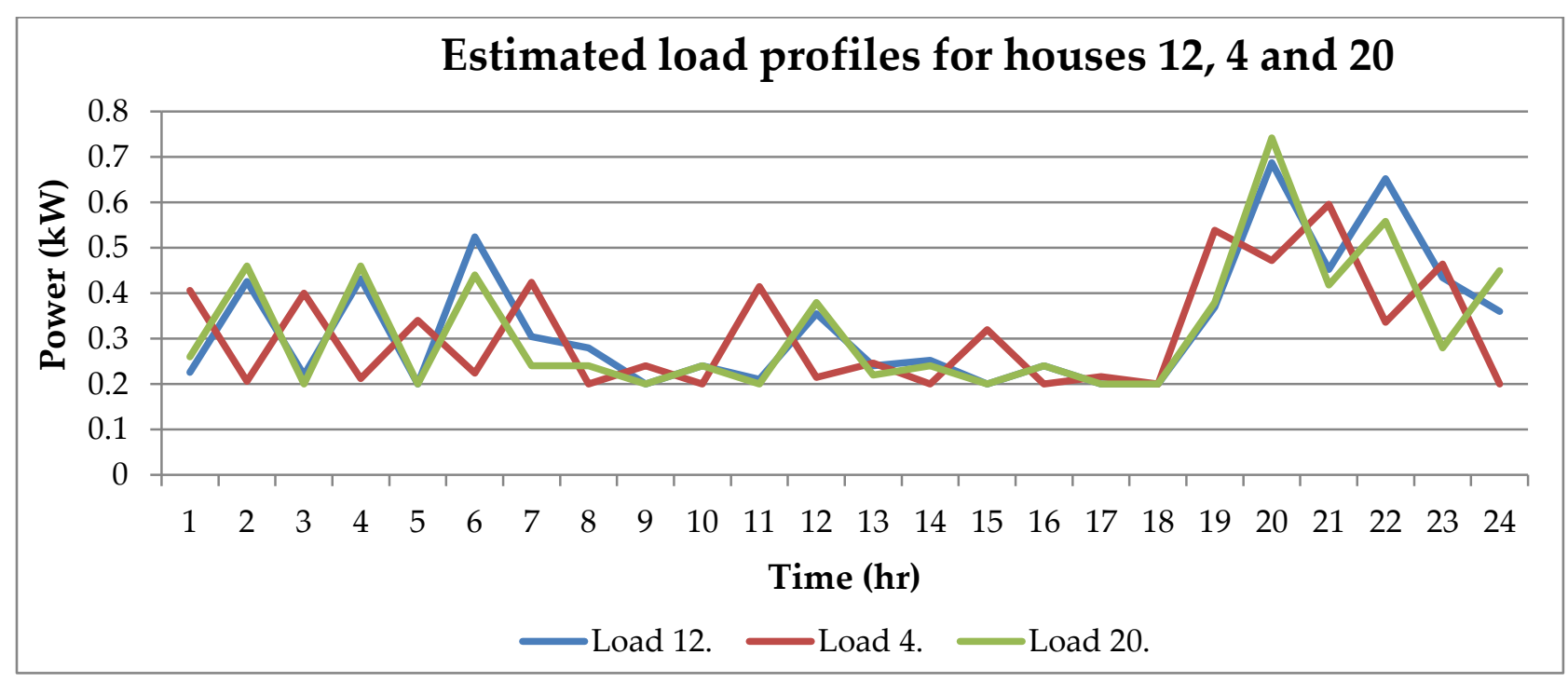

(f) 


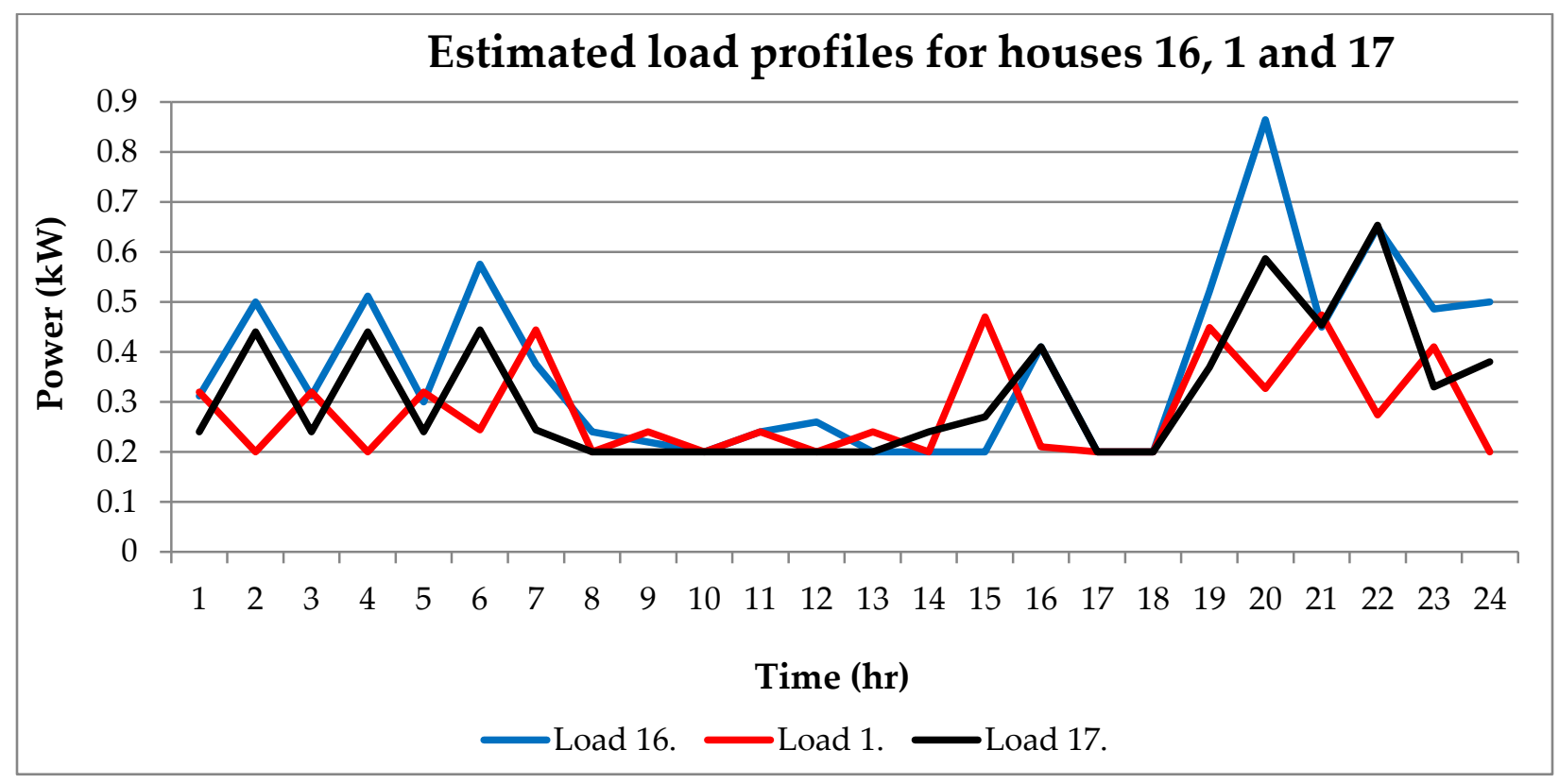

(g)

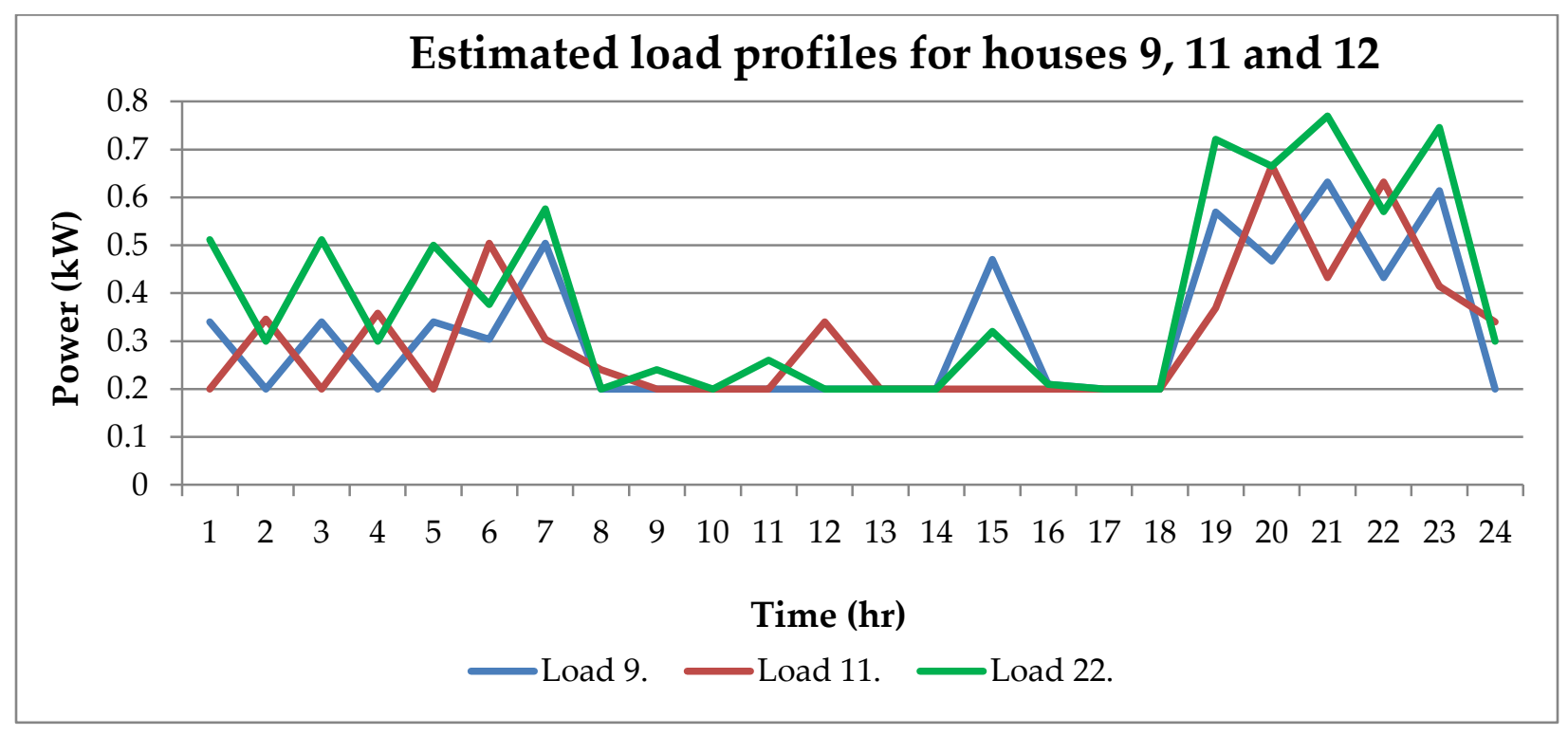

(h)

Figure 11(a) - (h): Estimated load profiles of the households 


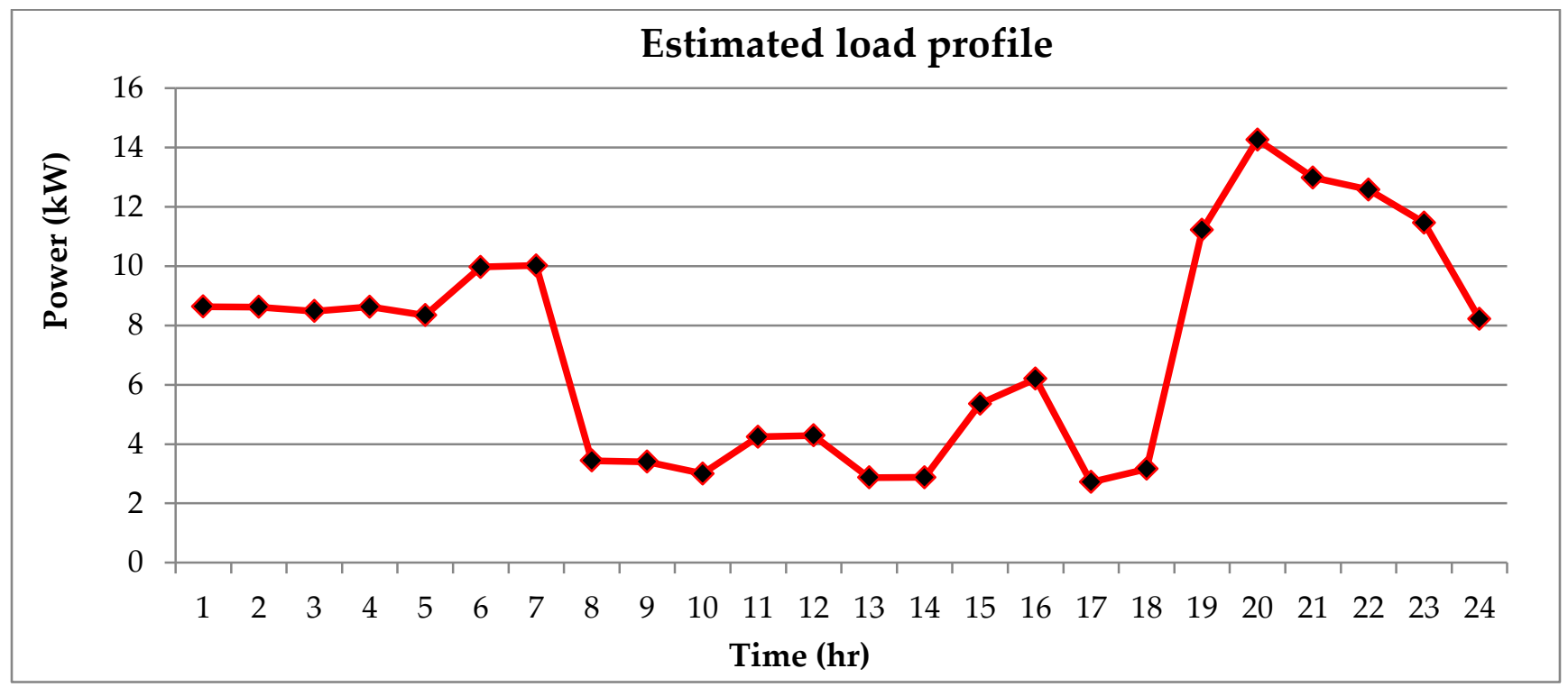

Figure 12: The community's estimated load profile

Table 8: Estimated loads and energy consumption of the houses

\begin{tabular}{ccc}
\hline House & Load $(\mathrm{kW})$ & Demand $(\mathrm{kWh})$ \\
\hline 1 & 0.737 & 5.702 \\
2 & 0.757 & 7.482 \\
3 & 0.797 & 6.092 \\
4 & 0.817 & 6.677 \\
5 & 0.859 & 6.498 \\
6 & 0.943 & 7.221 \\
7 & 0.703 & 7.182 \\
8 & 0.757 & 7.694 \\
9 & 0.783 & 6.442 \\
10 & 0.808 & 8.162 \\
11 & 0.823 & 6.379
\end{tabular}




\subsection{Solar energy resources of the locations}

The peak sun hours (PSH) of Nigeria's geo-political zones are presented in Figures 13 and 14. This basically represents the selected locations' average solar irradiation values in $\mathrm{kWh} / \mathrm{m}^{2}$ per day. The lowest PSH for the NE, NW, NC, SE, SS and SW are 5.24, 5.21, 3.84, 3.45, 3.46 and 4.5, respectively, while the highest $P S H$ are 6.67, 6.69, 6.63, 5.61, 4.63 and 5.98 [150]. It can be observed that the lowest and the highest PSH for the northern zones are 3.84 and 6.69, respectively, while the corresponding values for the southern zones are 3.45 and 5.98. This reveals that Nigeria's northern region has a higher solar energy potential compared to its southern region [150]. Also, the average ambient temperatures of the zones are shown in Figures 15 and 16, respectively. The lowest and the highest temperatures of the northern zones are $21.89{ }^{\circ} \mathrm{C}$ and $32.05{ }^{\circ} \mathrm{C}$, respectively, while the southern zones have the corresponding values of $24.48{ }^{\circ} \mathrm{C}$ and 
$28.15{ }^{\circ} \mathrm{C}$. It is clearly shown that the northern zones have relatively higher ambient temperature values.

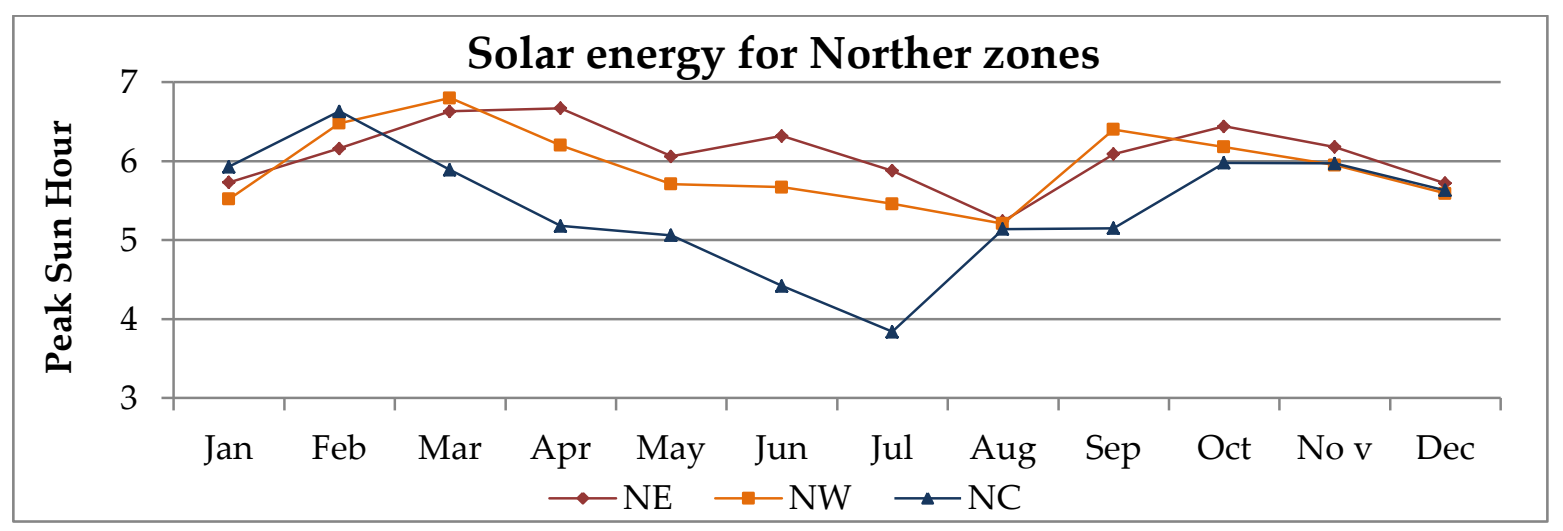

Figure 13: Peak sun hours of the northern zones [150]

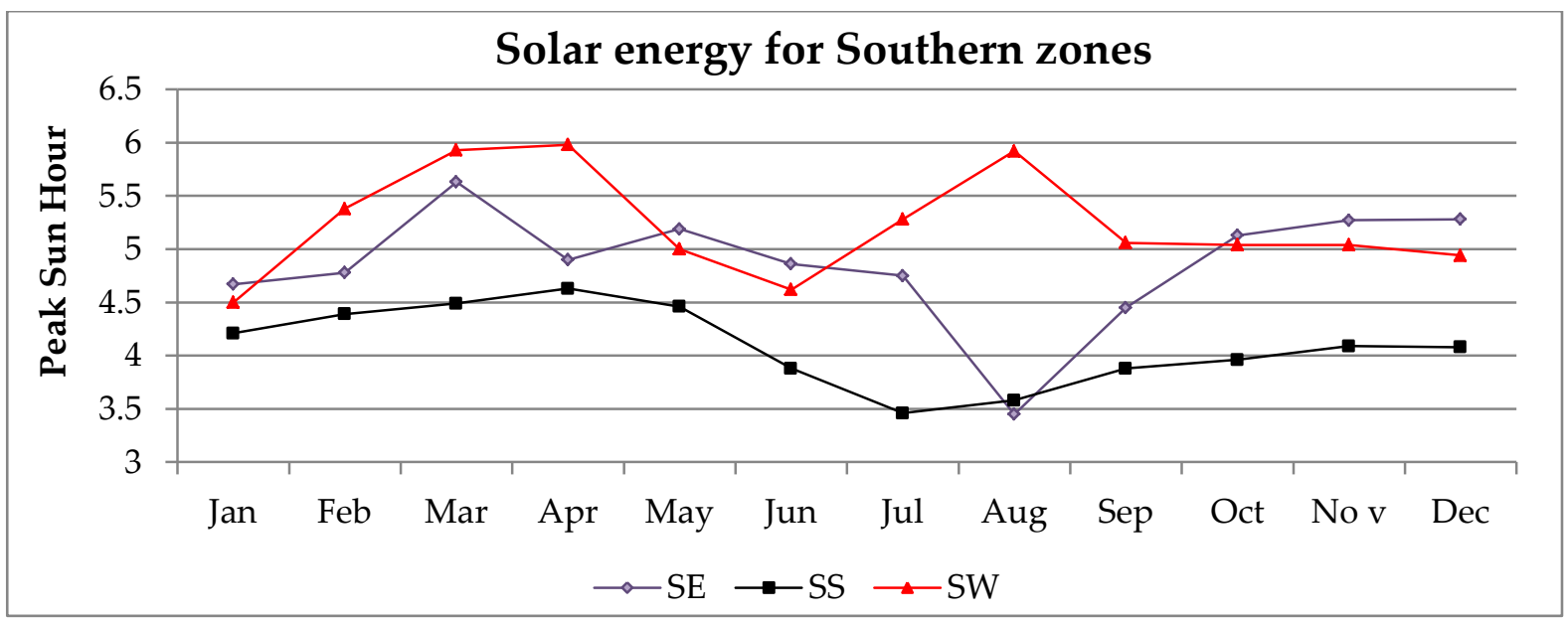

Figure 14: Peak sun hours of the southern zones [150] 


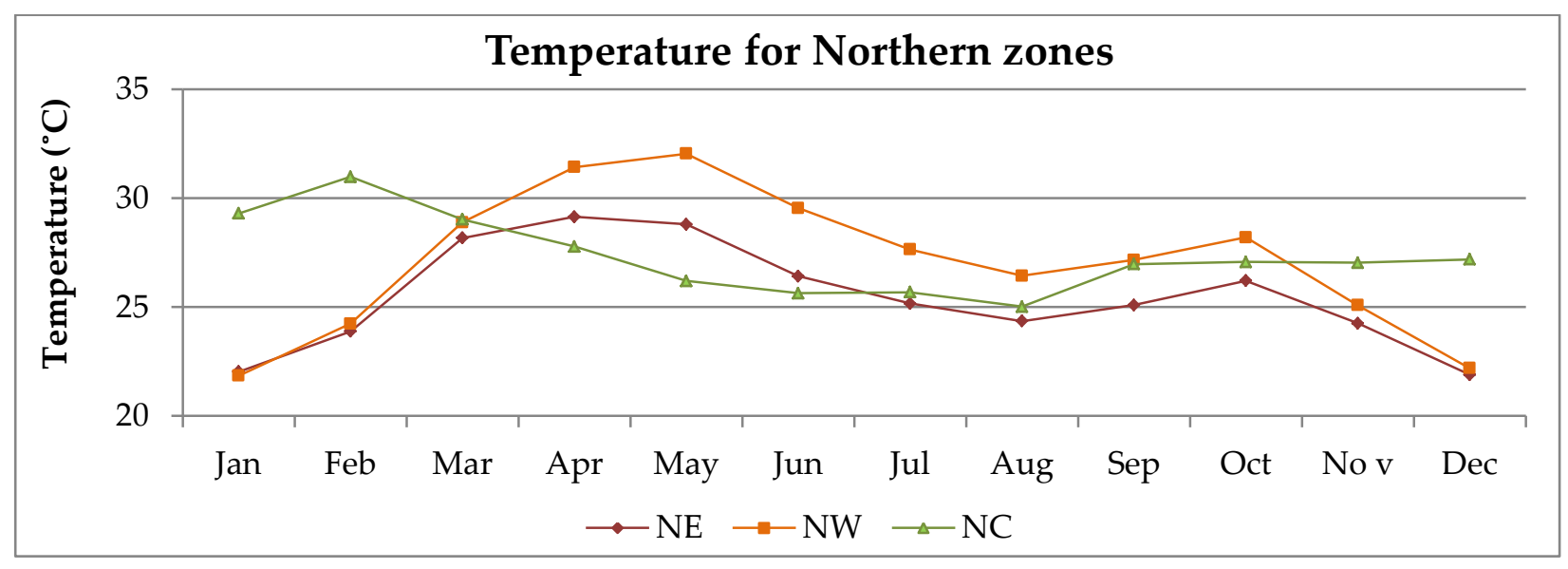

Figure 15: Ambient temperature of the northern zones [150]

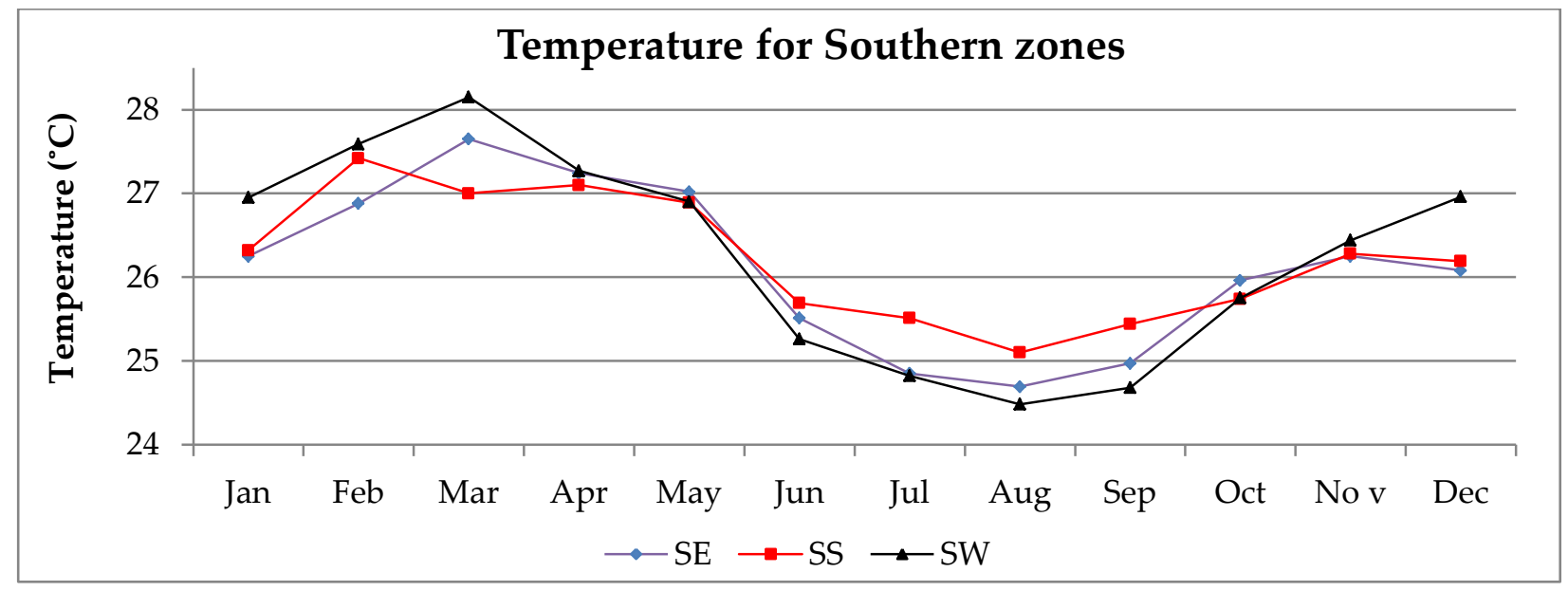

Figure 16: Ambient temperature of the southern zones [150]

\subsection{Microgrid model}

The architectural layout provided by the building consortium in Lagos, Nigeria [225], translates to the drawing presented in Figure 17 (a). The drawing provides the information on how the houses will be physically built on land. The distances of the houses to the solar photovoltaic microgrid system are analysed and presented as follows:

Branch A

House 1: $15000 \mathrm{~mm}=15 \mathrm{~m}$

House 2: $15000+14325+6000=35325 \mathrm{~mm}=35.325 \mathrm{~m}$

House 3: $15000+14325+6000+14325+6000=55650 \mathrm{~mm}=55.650 \mathrm{~m}$ 
House 4: $15000+14325+6000+14325+6000+14325+6000=75975 \mathrm{~mm}=75.975 \mathrm{~m}$ House 5: $(4)+14325+6000=96300 \mathrm{~mm}=96.3 \mathrm{~m}$

House 6: $(5)+6000+14325+14325=130950 \mathrm{~mm}=130.95 \mathrm{~m}$

Branch B \& C

House $1: 12000 \mathrm{~mm}=12 \mathrm{~m}$

House $2: 12000+(14325-8400)+8400+6000=32325 \mathrm{~mm}=32.325 \mathrm{~m}$

House 3: $(2)+8400+(14325-8400)+6000+(14325-8400)=58575 \mathrm{~mm}=58.575 \mathrm{~m}$

House 4: $(3)+8400+6000=72975 \mathrm{~mm}=72.975 \mathrm{~m}$

House 5: $(4)+8400+(14325-8400)+6000+(14325-8400)=99225 \mathrm{~mm}=99.225 \mathrm{~m}$

House 6: $(5)+8400+6000+8400=122025 \mathrm{~mm}=122.025 \mathrm{~m}$

Branch D

House 1: $22000 \mathrm{~mm}=22 \mathrm{~m}$

House 2: $(1)+(14325-8400)+8400+6000=42325 \mathrm{~mm}=42.325 \mathrm{~m}$

House 3: $(2)+8400+(14325-8400)+6000+(14325-8400)=68575 \mathrm{~mm}=68.575 \mathrm{~m}$

House 4: $(3)+8400+6000=82975 \mathrm{~mm}=82.975 \mathrm{~m}$

House 5: $(4)+8400+(14325-8400)+6000+(14325-8400)=109225 \mathrm{~mm}=109.225 \mathrm{~m}$

House $6:(5)+8400+6000+8400=132025 \mathrm{~mm}=132.025 \mathrm{~m}$

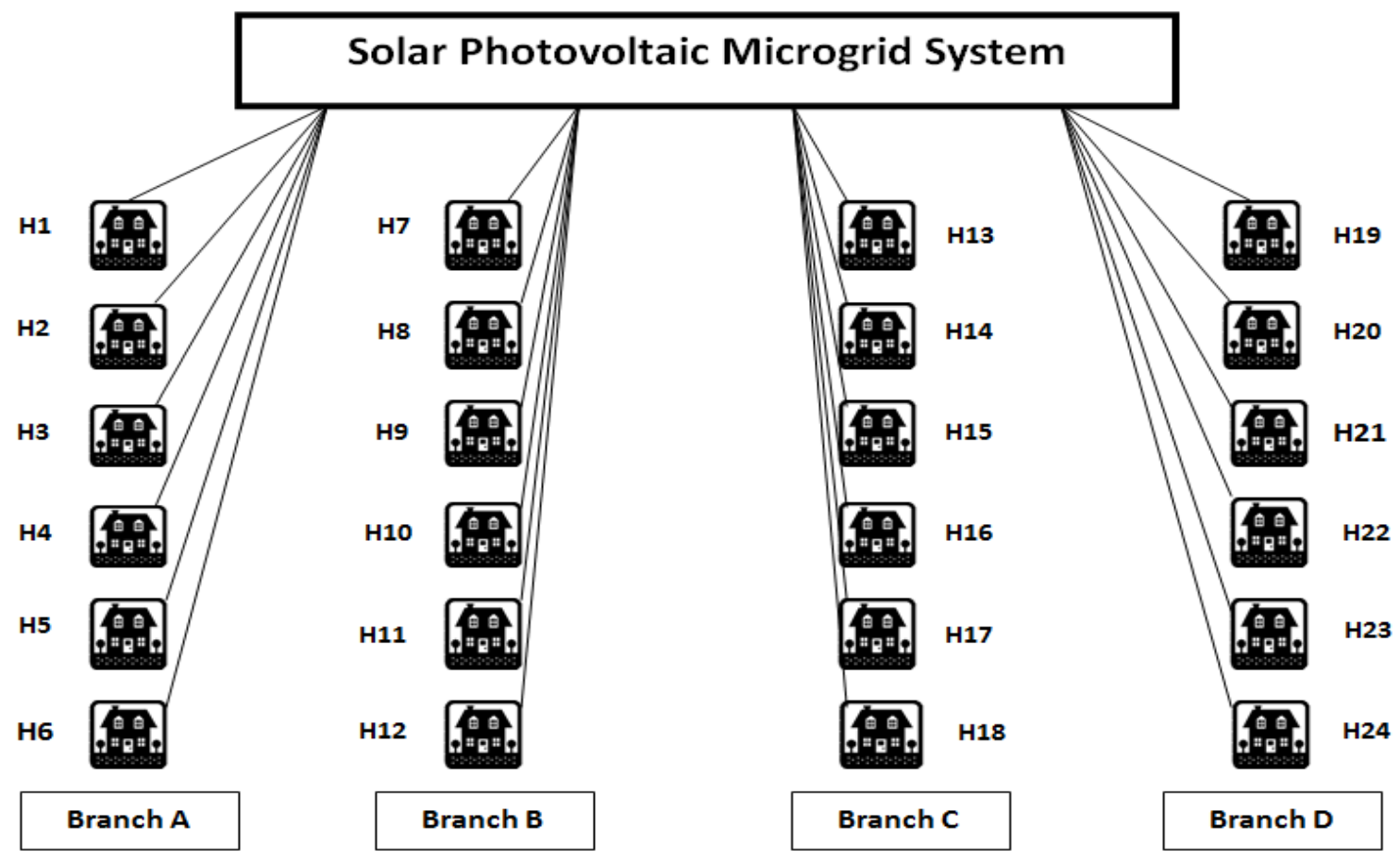

(a) 


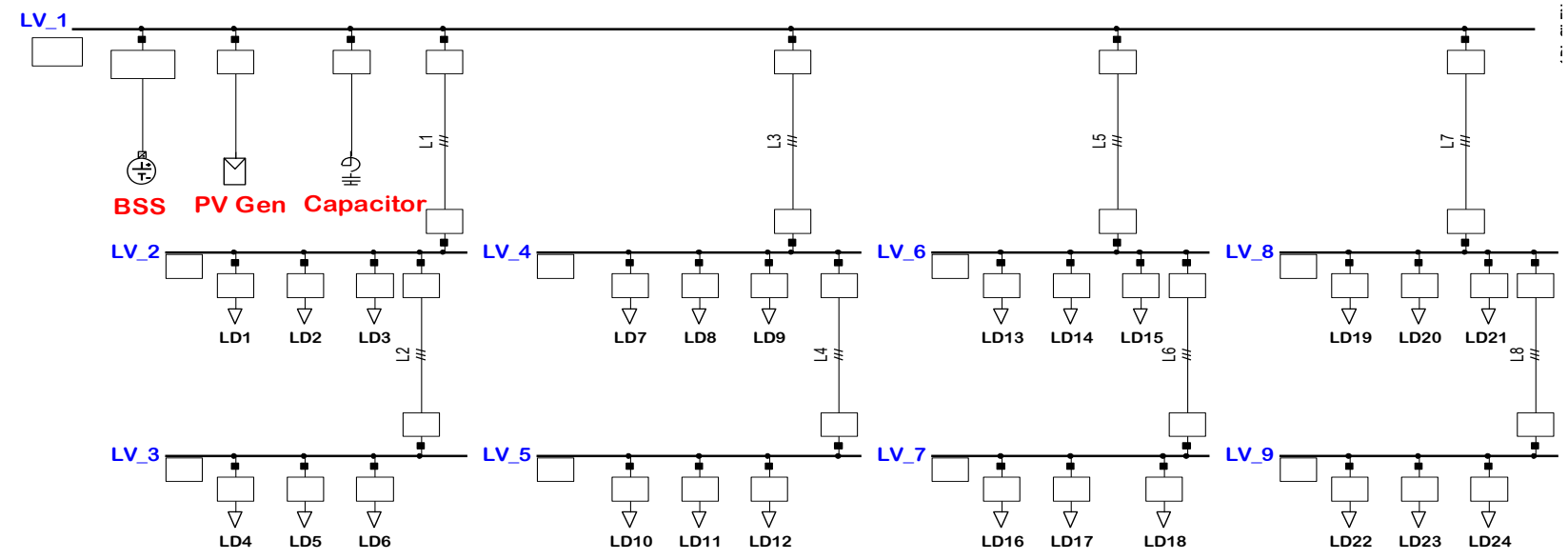

(b)

Figure 17(a) Schematic of the community (b) SPM model

where:

$P V$ Gen is the solar photovoltaic array

$B S S$ is the battery storage system

$L V \_1$ to $L V \_9$ are the low-voltage busbars

$L 1$ to $L 8$ are the electric lines

$L D 1$ to $L D 24$ are the loads in the houses

The total lengths of branches A, B, C and D are $130.95 \mathrm{~m}, 122.025 \mathrm{~m}, 122.025 \mathrm{~m}$ and $132.025 \mathrm{~m}$, respectively. The single-phase users' loads presented in Table 8 are arranged on the three-phase output of the solar photovoltaic microgrid systems, and the arrangement is shown in Table 9. This information and the location's solar irradiance and ambient temperature are introduced to the SPM model. The results of a series of load flow calculations detailing the SPM's energy production, busbar voltages, and the line losses are presented in Chapter 5.

Table 9: Arrangement of loads in the LV network

\begin{tabular}{l|l|l}
\hline Loads on Phase a $(\mathrm{kW})$ & Loads on Phase $\mathrm{b}(\mathrm{kW})$ & Loads on Phase c $(\mathrm{kW})$ \\
\hline Load $1 \rightarrow 0.737$ & Load2 $\rightarrow 0.757$ & Load3 $\rightarrow 0.797$ \\
Load4 $\rightarrow 0.817$ & Load5 $\rightarrow 0.859$ & Load6 $\rightarrow 0.943$
\end{tabular}




\begin{tabular}{|c|c|c|}
\hline Load7 $\rightarrow 0.703$ & Load8 $\rightarrow 0.757$ & Load9 $\rightarrow 0.783$ \\
\hline Load10 $\rightarrow 0.808$ & Load11 $\rightarrow 0.823$ & Load12 $\rightarrow 0.878$ \\
\hline Load13 $\rightarrow 0.663$ & Load14 $\rightarrow 0.724$ & Load15 $\rightarrow 0.789$ \\
\hline Load16 $\rightarrow 0.799$ & Load17 $\rightarrow 0.849$ & Load18 $\rightarrow 0.955$ \\
\hline Load19 $\rightarrow 0.714$ & Load20 $\rightarrow 0.734$ & Load21 $\rightarrow 0.804$ \\
\hline Load22 $\rightarrow 0.838$ & Load23 $\rightarrow 0.955$ & Load24 $\rightarrow 0.973$ \\
\hline 6.079 & 6.458 & 6.922 \\
\hline
\end{tabular}

\subsection{Chapter summary}

This chapter has presented six different locations as case studies for the proposed solar photovoltaic microgrid systems, one from each of Nigeria's six geo-political zones. The country's geographical characteristics and situation, i.e. coordinates, location, land mass, climate, solar energy resources and the current electricity status were discussed.

In addition, the chapter has presented the categories of people for whom the solar photovoltaic models are proposed, their daily lifestyles, electricity consumption and how these are instrumental in developing the community's aggregate load profile.

The selected communities' peak sun hours and ambient temperatures were highlighted. Furthermore, the schematic diagram of the proposed solar photovoltaic microgrid model was presented. The model has been developed using the DIgSILENT PowerFactory simulation tool based on the community's layout. The distances of the houses to the proposed power plant, the solar irradiance and temperature, and the different household single-phase load models are among the major data included in the model. The next chapter discusses the results and analysis of the SPM systems with no users' load demand growth. 


\section{Chapter 5}

\section{Microgrid analysis with no load growth}

This chapter presents the scenarios, detailed results and analyses of the solar photovoltaic microgrid systems with no users' load demand growth. It begins by evaluating the capacities of the proposed energy system's main components, considering the design parameters that have been discussed in Chapters 3 and 4 . This is then followed by assessing the power and energy outputs, energy losses and efficiency, battery state of charge, unmet load, loss of energy probability and the availability parameters.

The results for three different scenarios are presented (i.e. the initial scenario, scenario 1 and scenario 2). The initial scenario serves as the baseline; scenario 1 focuses on the increase in the SPM's capacities, while scenario 2 concentrates on users' load demand reduction. Both the scenarios 1 and 2 ensure that the SPM's battery constraints are within the acceptable limits, according to the design strategy presented in Chapter 3. These scenarios are intended to achieve microgrid systems with good reliability indices, i.e. those with high availability and low loss of energy probability.

The chapter is divided into four main parts. The first part presents the results and analyses of the system component capacities; this includes the SPM's rated power capacities, the energy outputs, yield and losses, and the monthly average efficiencies. The second part provides the results and assessments of the energy storage systems for the different locations, while the third section focuses on the hourly energy balance variations and detailed reliability analyses. The fourth part reports the different location-specific design safety factors, which are proposed for determining suitable SPM sizes for the specified locations. A summary concludes the chapter.

\subsection{System capacity and electrical performance}

\subsubsection{Component sizes}

The proposed SPM's sizes for the different communities are presented in Table 10. The difference in the system's capacities is primarily due to the disparities in the solar energy potential of the locations. The battery capacity for the load demand of 
$174.98 \mathrm{kWh}$ at a system voltage of $348 \mathrm{~V}$, DoD of $50 \%$ and the autonomy of $\sim 1.5$ is 540.24 $\mathrm{kWh}$. The inverter/controller of $60 \mathrm{kVA}$ is also selected for the users' total load capacity of $\sim 19.50 \mathrm{~kW}$ [7]. The battery and the inverter capacities have been obtained according to Eqs. (28) and (36) in Chapter 3.

The SPM's baseline capacities are presented in Table 10. The NASENI 190W mono c-Si module $[158,220]$ is used for analysing the yearly energy production and yield of the proposed SPMs. This PV module is locally fabricated in the country by the National Agency for Science and Engineering Infrastructure (NASENI), under the supervision of the Federal Ministry of Science and Technology (FMST), Abuja, Nigeria. The module is selected due to its relatively lower cost and availability, compared to the imported solar PV modules. The solar module parameters are shown in Table 11. It can be seen that it has the highest voltage of $44.51 \mathrm{~V}$, i.e. $V_{o c}$, but due to the temperature effects, this value drops to a minimum of $\sim 27$ to $32 \mathrm{~V}$ as shown in Figure 18. The corresponding values of $T_{a}$ and $T_{c}$ are presented in Figures 19 and 20, respectively. These analyses are based on the methodologies discussed in Chapter 3.

The literature demonstrates that a day in the dry season is usually selected to evaluate the variation between the PV energy generation and the demand $[13,15,18,19$, 131]. However, a detailed analysis that considers all the seasons in the year, i.e. dry and rainy seasons is necessary for adequacy, reliability and decision-making purposes. The days selected for the zones are $3^{\text {rd }}$ April, $1^{\text {st }}$ March, $28^{\text {th }}$ February, $4^{\text {th }}$ April, $18^{\text {th }}$ April and $6^{\text {th }}$ April, respectively. These days fall within the dry season in all the zones. A designer may decide to select any other day during the dry season apart from the selected days. What is necessary is to obtain an initial daily energy balance assessment of the solar PV energy output and the users' energy usage, before evaluating the monthly and the yearly performances $[139,141]$.

The baseline voltage in Figure 18 represents the module maximum power voltage $\left(V_{m p p}\right)$ at $S T C$, while the other voltage values represent the voltages obtained at the actual module cell temperature. The STC specifies the module electrical performance at solar irradiance, air mass (A.M) spectral distribution, and cell temperature of $1 \mathrm{~kW} / \mathrm{m}^{2}$, 1.5 and $25^{\circ} \mathrm{C}$, respectively $[6,13,18,19,56,69,151,153,172-176]$.

However, because such a test condition is based on the ideal situations for manufacturing purposes, it, therefore, overestimates the real-world performance. This is 
because the solar PV cell temperature (i.e. $T_{c}$ ) and the irradiance (i.e. $G_{a}$ ) are rarely at a respective value of $25{ }^{\circ} \mathrm{C}$ and $1 \mathrm{~kW} / \mathrm{m}^{2}$, at the same time. Thus, the irradiance values for $T_{c} \leq 25$ are usually less than $1 \mathrm{~kW} / \mathrm{m}^{2}$ because such values are usually reached in the afternoon in most tropical regions. They are usually associated with a temperature rise. This factor reduces the voltage, power output and the efficiency $[6,7,13,56,69,151$, 153, 154, 172-176]. This is clearly demonstrated by Figures 18 and 21-23.

Furthermore, the analysis presented in this thesis considers the solar PV system losses. Such an analysis presents a better prediction of the overall system output, compared to several studies in the literature that do not consider the system losses. The module minimum voltage is used for evaluating the power output when $T_{a}$ or $T_{c}$ is at the highest value in the day. The maximum power outputs of the $190 \mathrm{~W}$ module for the specified zones are shown in Figure 21, based on the values of $V_{\text {mpp }}$ of Figure 18. The maximum values of $158.42 \mathrm{~W}, 152.41 \mathrm{~W}, 156.48 \mathrm{~W}, 126.25 \mathrm{~W}, 120.04 \mathrm{~W}$ and $154.59 \mathrm{~W}$ are obtained for NE, NW, NC, SE, SS and SW zones, respectively. These values are lower than the rated module power of $190 \mathrm{~W}$ as a result of the temperature effect. It can be clearly seen in Figure 18 to 23 that as $T_{a}$ increases, $T_{c}$ also increases, while $V_{m p p}, P_{m p p}$ and the efficiency reduces.

In addition, the other losses are also evaluated. This is achieved through the $d_{\text {rf }}$ $[7,136,152,154,158]$. This factor considers the losses due to dust, wiring, inverter losses etc. A $d_{r f}$ of 0.85 is used in this thesis, which is obtained from the different de-rating parameters shown in Table 12. Similar values are also reported in [177]. The PV module is, therefore, de-rated by this factor to account for the other losses (O.L), apart from the losses due to temperature effect (L. D. T. T. E). After considering the other losses, the final output of the $190 \mathrm{~W}$ PV module is shown in Figure 22. The maximum values of 134.65 W, 129.55 W, 133.01 W, 107.31 W, 102.04 W and 131.40 W are obtained for NE, NW, NC, SE, SS and SW zones, respectively. The efficiency over the 24-hour period is also presented in Figure 23. It is obvious that the module efficiencies are dependent on the voltage and the power output.

In addition, 174 units of $2 \mathrm{~V} 800 \mathrm{Ah}$ cell will be required for the $540.24 \mathrm{kWh}$ battery bank at a system voltage of $348 \mathrm{~V}$. The $2 \mathrm{~V}$ battery cell basically requires a bulk charge voltage of $2.4 \mathrm{~V}$ [7], therefore, 174 units of the battery cell will need a bulk charge voltage of $417.6 \mathrm{~V}$ for a system voltage of $348 \mathrm{~V}$. The highest number of modules that 
can be wired in series is 15 , translating to a total voltage of $15 \times 44.51 \mathrm{~V}=668 \mathrm{~V}$ from the $\mathrm{PV}$ array for all the locations. An ETS-60kVA inverter/controller is selected for the analysis [7]. This inverter system has been designed to accommodate the highest $V_{o c}$ of $690-700 \mathrm{~V}$, and the lowest value of $390 \mathrm{~V}$. The total voltage of $668 \mathrm{~V}$ is suitable for this inverter input capability. The minimum array voltage differs from location to location because of the temperature effects. This is calculated as the product of the minimum voltage and the number of modules in series. This is considered in the model shown in Figure 17.

Table 10: SPM sizes for the communities

\begin{tabular}{cc}
\hline Location & SPM capacity $(\mathbf{k W})$ \\
\hline NE & 40.07 \\
NW & 40.30 \\
NC & 54.15 \\
SE & 60.86 \\
SS & 60.87 \\
SW & 46.66 \\
\hline
\end{tabular}

Table 11: Parameters of the NASENI 190W module

\begin{tabular}{ccccccc}
\hline $\boldsymbol{P}_{m p p}(\mathrm{~W})$ & $\boldsymbol{I}_{s c}(\mathrm{~A})$ & $\boldsymbol{V}_{o c}(\mathrm{~V})$ & $\boldsymbol{V}_{m p p}(\mathrm{~V})$ & $\boldsymbol{I}_{m p p}(\mathrm{~A})$ & $\boldsymbol{\eta}(\mathbf{\%})$ & Max. Sys. Voltage (V) \\
\hline 190 & 5.498 & 44.51 & 36.17 & 5.259 & 14.9 & $1000 \mathrm{~V}$ DC \\
\hline
\end{tabular}




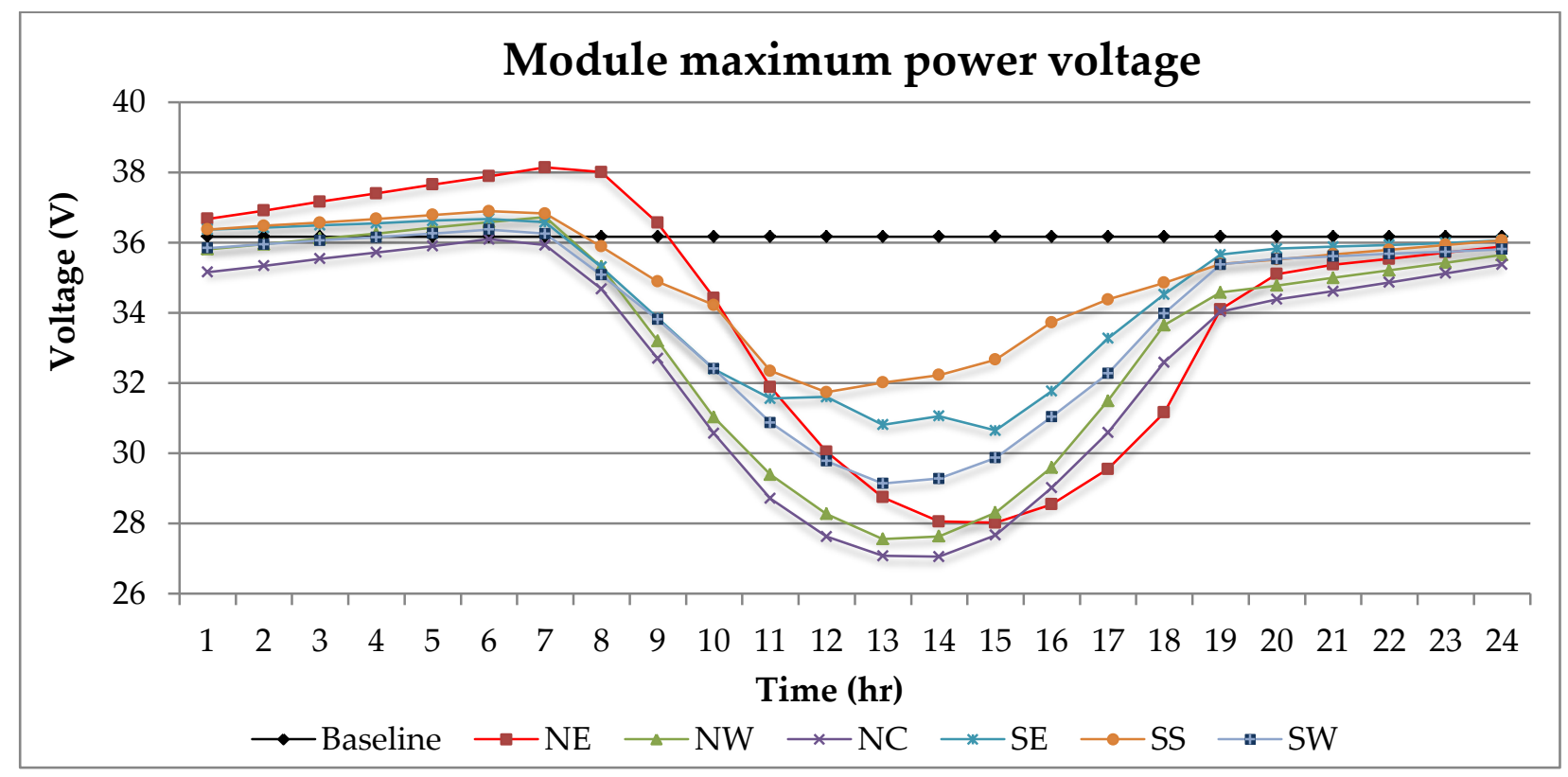

Figure 18: Actual $V_{m p p}$ values for the zones

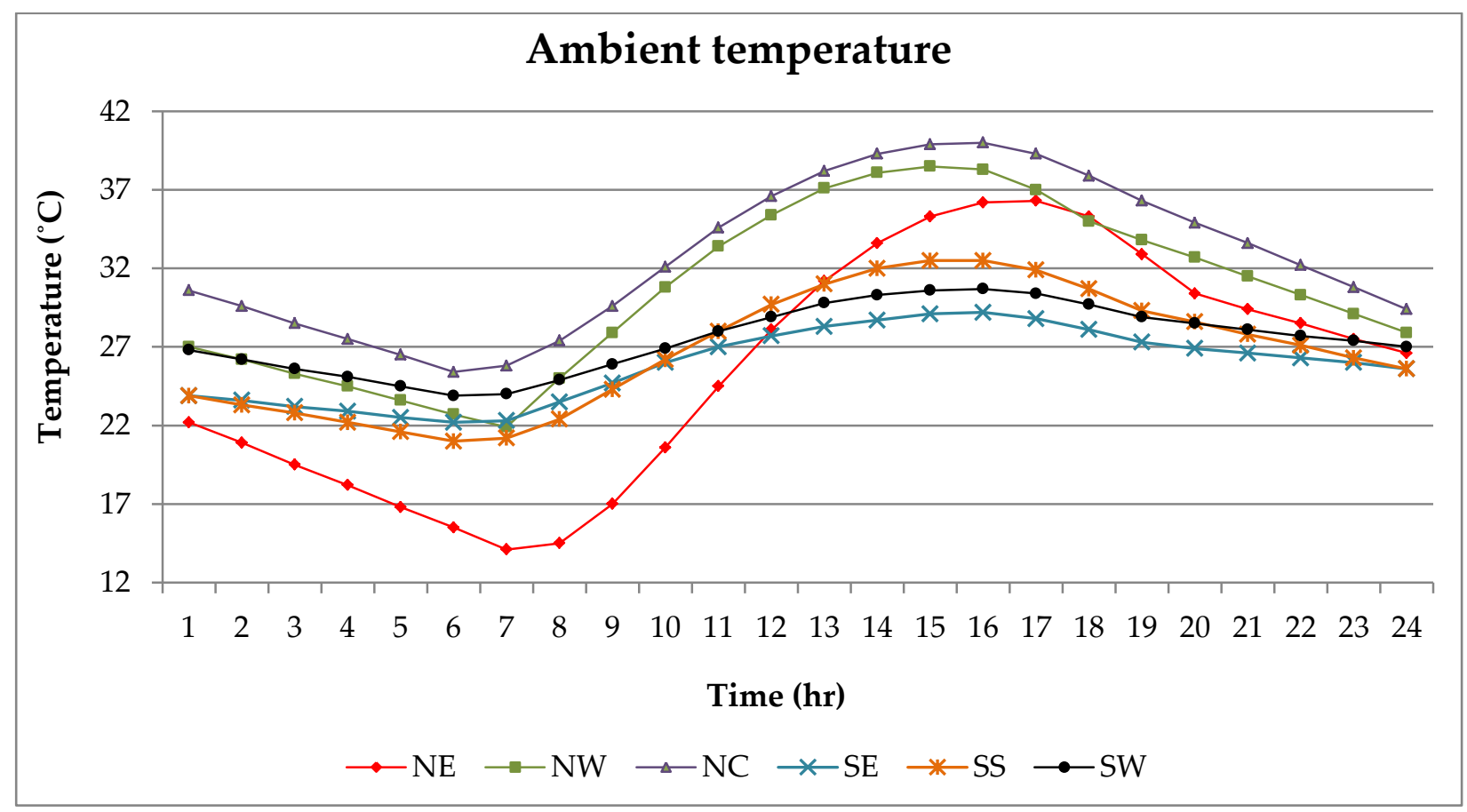

Figure 19: Corresponding ambient temperatures for the zones [150] 


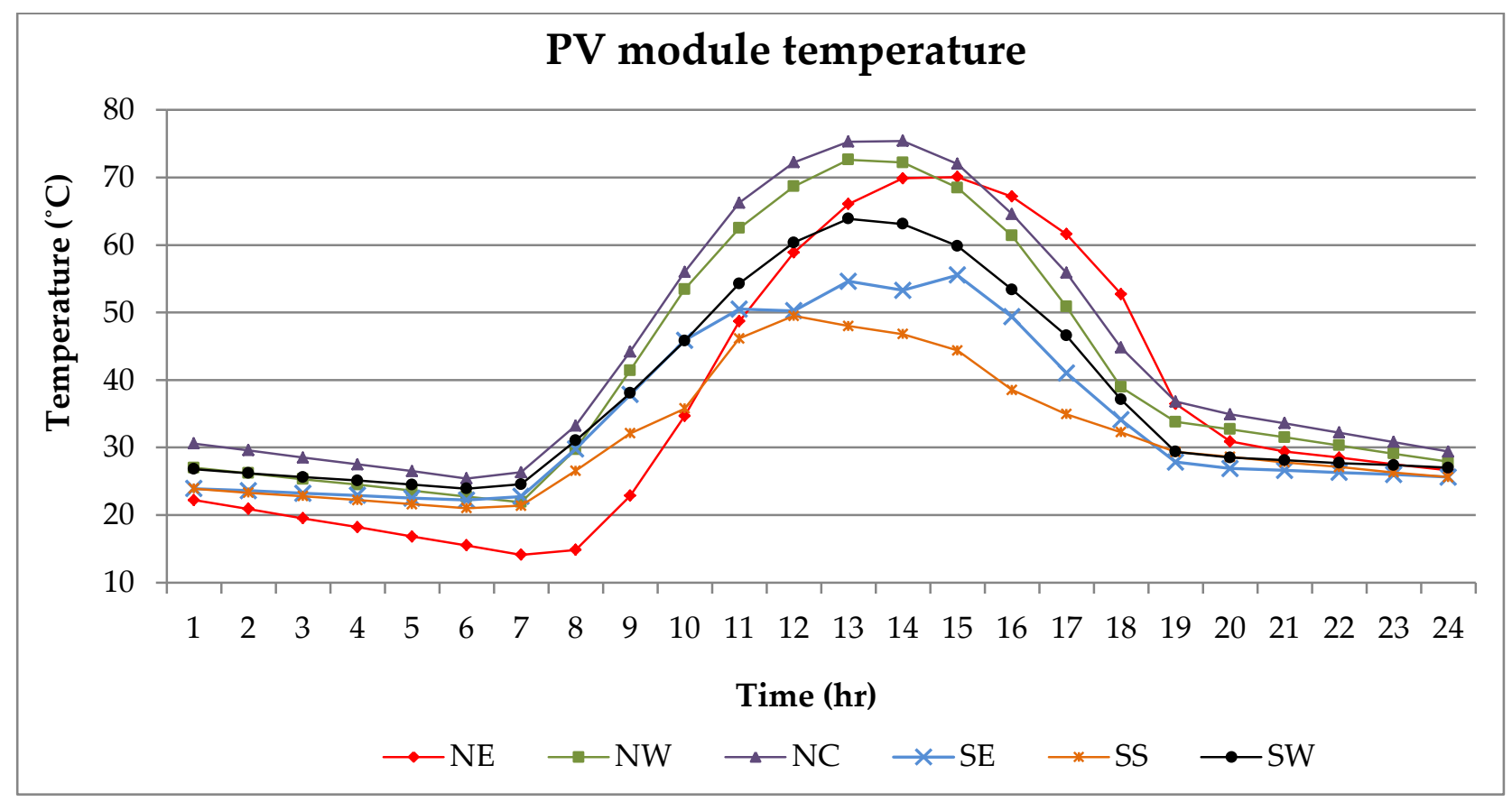

Figure 20: Corresponding PV module temperatures for the zones

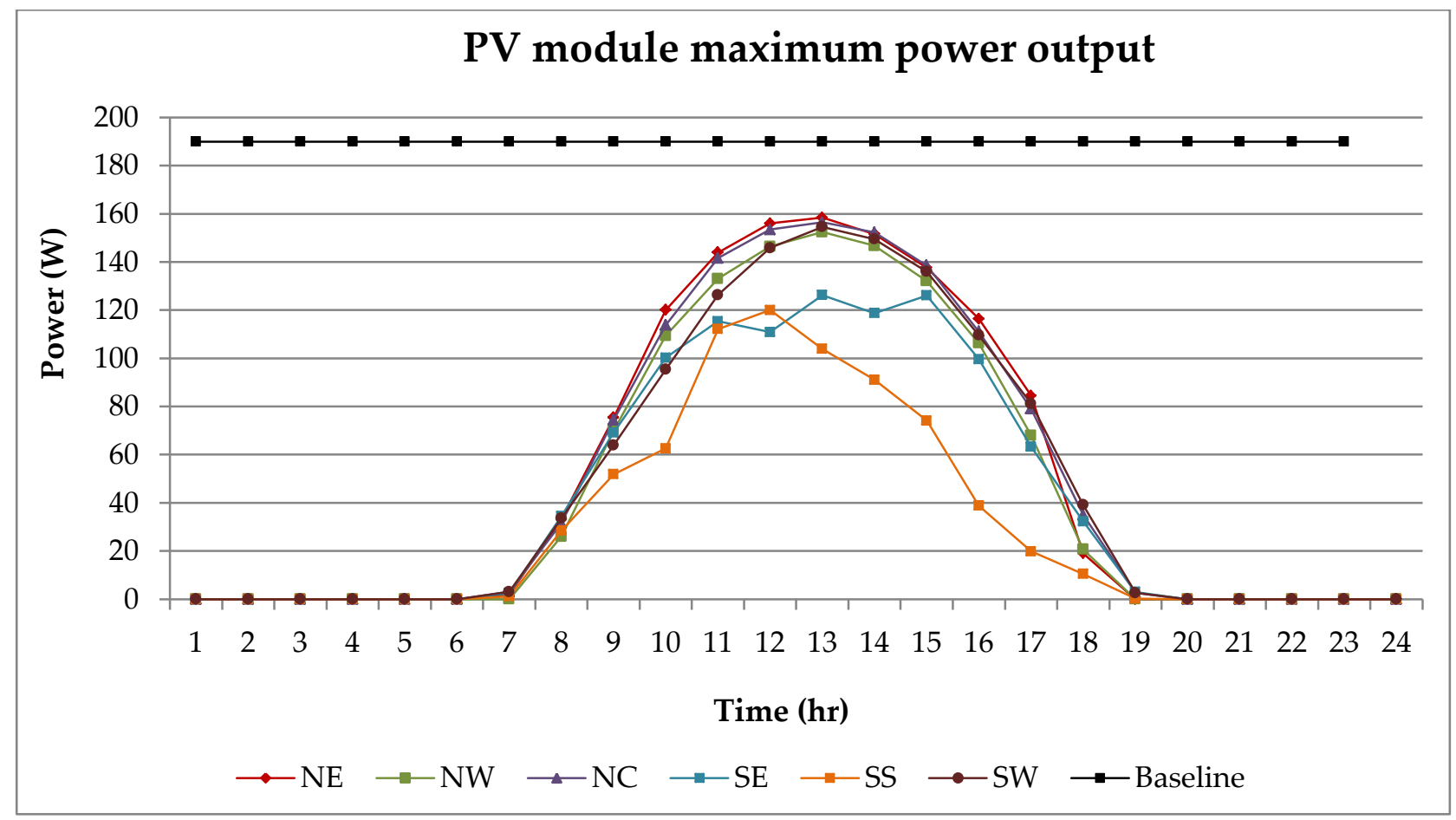

Figure 21: 190W module power output after temperature losses 
Table 12: The de-rating factor

\begin{tabular}{lc}
\hline Parameters & Derating values \\
\hline Inverter & 0.96 \\
Mismatch & 0.98 \\
Wiring & 0.97 \\
Soiling & 0.96 \\
Shading & 0.97 \\
\hline Overall & $\mathbf{0 . 8 5}$ \\
\hline
\end{tabular}

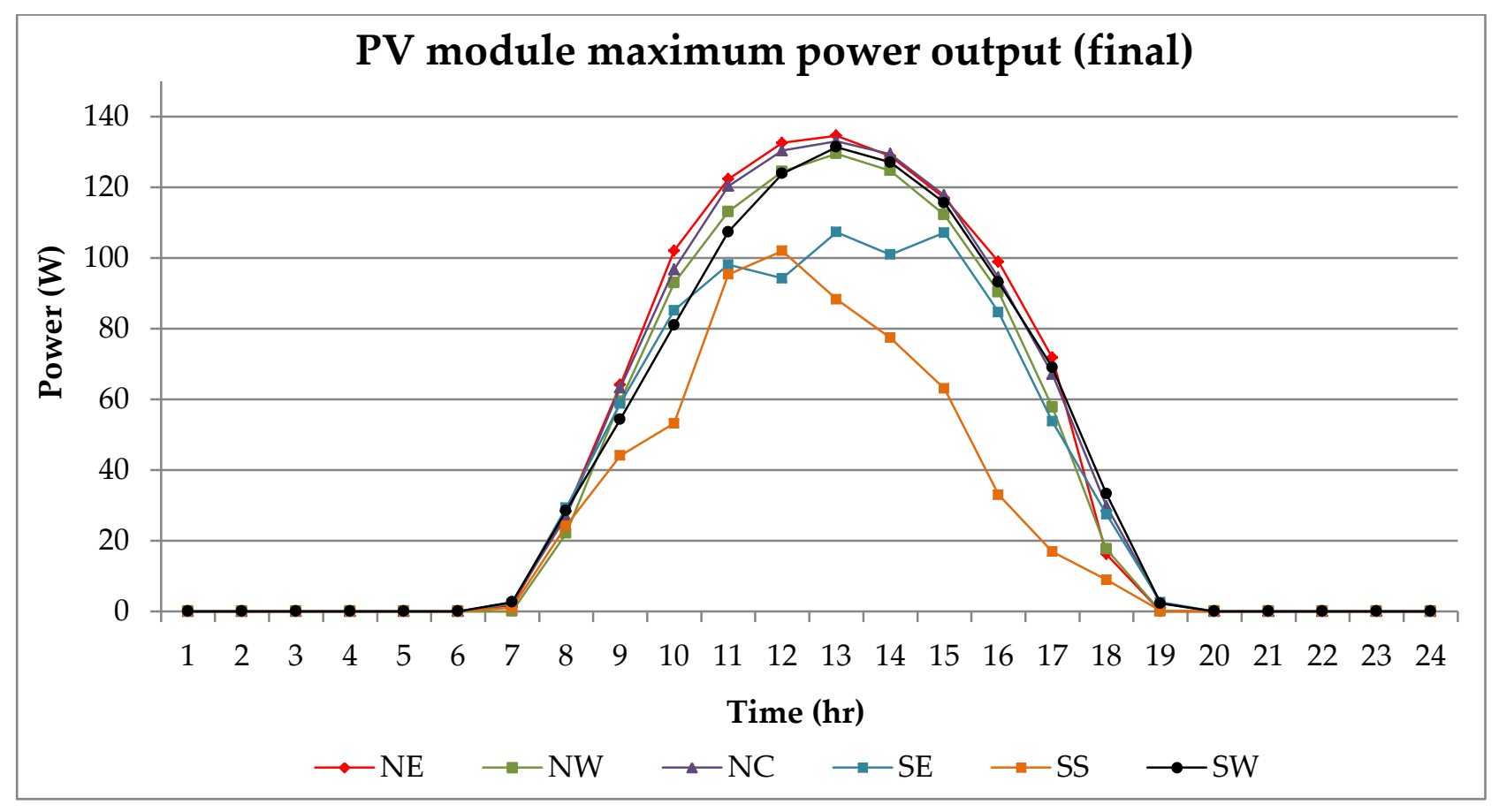

Figure 22: $190 \mathrm{~W}$ module power output after all losses are considered 


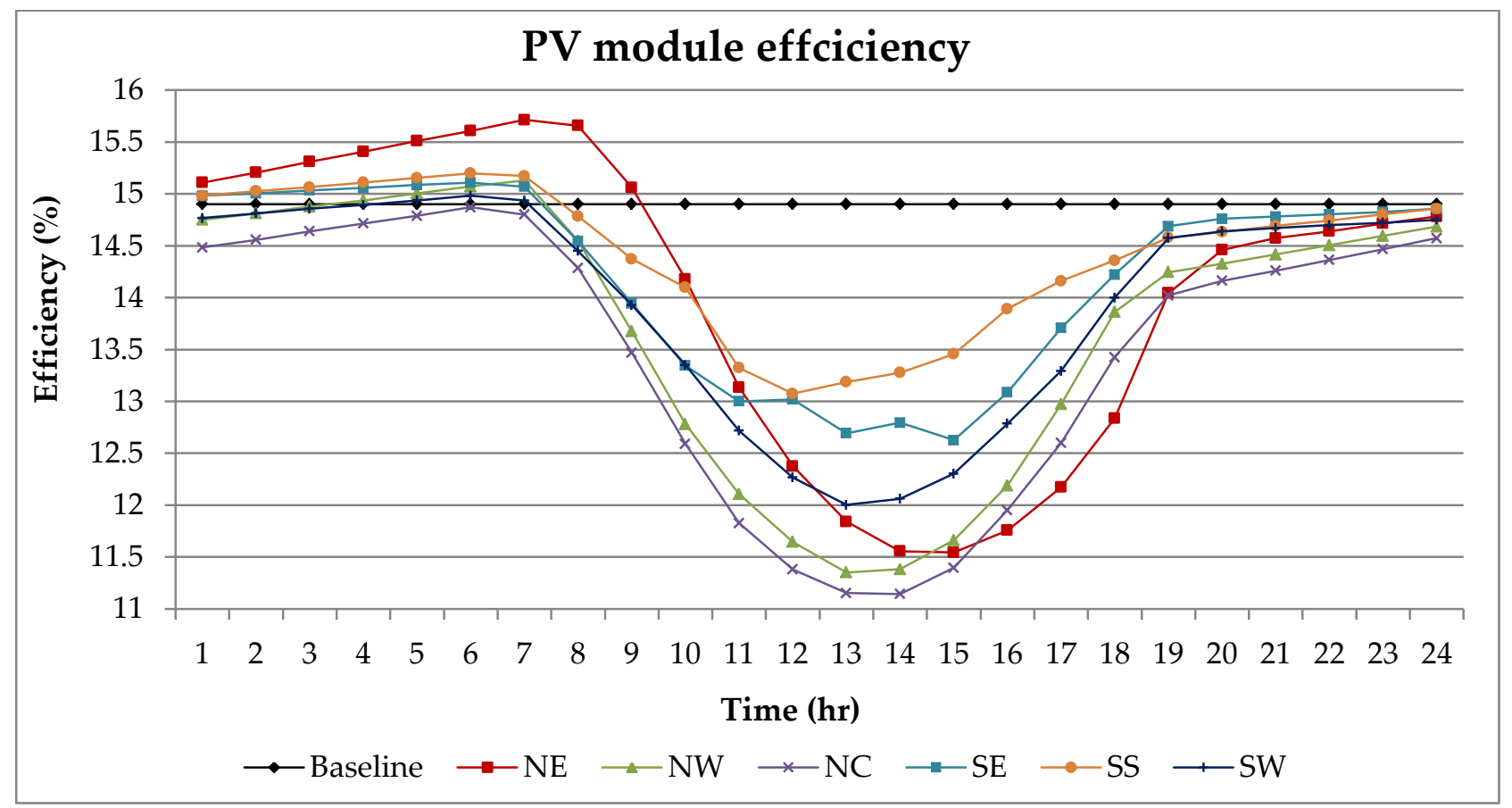

Figure 23: Efficiency of the PV module for the zones

\subsubsection{Daily power and energy outputs}

\section{1) NE location}

The result of the load flow calculations for the selected day is presented in Figure 24. The PV Power (Baseline), PV Power (A. T. L) and the PV Power (A. O. L) represent the rated $\mathrm{PV}$ power, $\mathrm{PV}$ power after considering temperature losses and the PV power after considering the other losses, respectively. The energy produced by the PV system over a 24-hour period, based on these parameters is $317.96 \mathrm{kWh}, 253.26 \mathrm{kWh}$ and 215.27 $\mathrm{kWh}$, respectively. The total losses are obtained from the difference between the baseline and the A. O. L value, which is $102.69 \mathrm{kWh}$ (i.e. $32.30 \%$ of the baseline). The losses due to temperature (L.D.T. T. E) and the other losses $(O . L)$ are therefore 64.7 $\mathrm{kWh}$ and $37.99 \mathrm{kWh}$, which are $20.35 \%$ and $11.95 \%$ of the baseline value, respectively.

These results demonstrate that $63 \%$ of the total losses are due to the temperature effect - an indication of the critical importance of considering the ambient temperature of any location when planning and designing PV energy systems. An increase in the ambient temperature $\left(T_{a}\right)$ decreases the PV system's maximum power voltage $\left(V_{m p p}\right)$, leading to a reduction in its maximum power output $\left(P_{m p p}\right)$ and the efficiency $(\eta)$. 
For instance, at $2 \mathrm{p} . \mathrm{m} ., T_{a}$ is $35.2{ }^{\circ} \mathrm{C}$ and this raises the cell temperature, $T_{c}$ to $71.48^{\circ} \mathrm{C}$, at the solar irradiance, $\mathrm{G}_{a}$ of $1.1 \mathrm{~kW} / \mathrm{m}^{2}$, according to Eq. (14). This is because the cell temperature can be $25-35^{\circ} \mathrm{C}$ hotter than the ambient temperature $[6,18,19$, 153]. Other solar PV research studies concentrate on the radiation and thermal modelling based on experimental analysis, with the intent of evaluating uncertainty in array temperature [226 - 228]. However, this thesis does not focus on radiation and thermal modelling. It uses the standard equations in the literature to analyse what is likely to be the effect of temperature on the proposed PV microgrids [152] when they are implemented in the future.

Also, at 8 a.m. $T_{a}$ and $T_{c}$ are $14.5^{\circ} \mathrm{C}$ and $17.3^{\circ} \mathrm{C}$, respectively, at the $G_{a}$ of $0.012 \mathrm{~kW} / \mathrm{m}^{2}$; at 7p.m. $T_{a}$ and $T_{c}$ are $32.9^{\circ} \mathrm{C}$ and $37.68^{\circ} \mathrm{C}$, respectively, at $G_{a}$ of $0.152 \mathrm{~kW} / \mathrm{m}^{2}$. These show the daily variation of the locations' irradiance and the ambient temperature. It can be seen that the cell temperature is higher in the afternoon compared to the morning and evening times. Therefore, the higher the difference between $T_{a}$ and $T_{\text {stc }}$, the higher the losses due to temperature effect $\left(T_{s t c}=25^{\circ} \mathrm{C}\right)$. This is the case in the afternoon. From this analysis, the energy available to support the users' load demand of $174.98 \mathrm{kWh}$ is, therefore, $215.27 \mathrm{kWh}$.

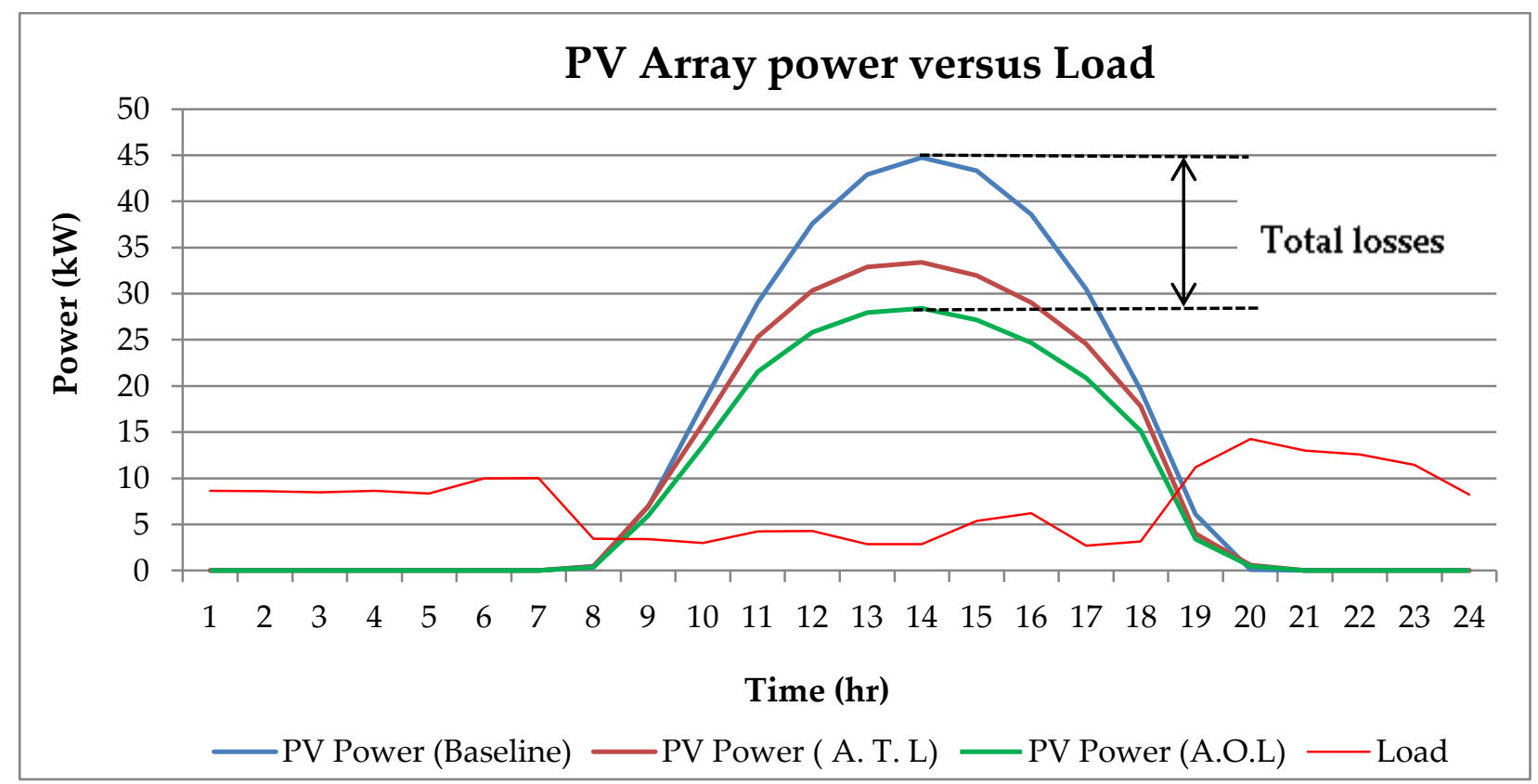

Figure 24: PV array power versus load demand for NE zone 
The system's busbar voltages with no capacitor are presented in Figure 25. The results show the voltage values of 0.672-1.293 p.u. These are found to be outside the specified steady state voltage range. This is because the single phase models in the network have the potential to give rise to voltage imbalance. To address this problem, a $10 \mathrm{kVar}$ reactive power compensation generator (i.e. capacitor) is included in the SPM model. This reactive power compensator has a degree and a maximum number of steps of $7 \%$ and 10, respectively. This same capacitor rating and settings are used for the energy systems considered for other locations. The system's busbar voltages with the capacitor bank are shown in Figure 26, with the minimum and maximum values of 0.952 and 1.031p.u, respectively. The line losses have also been minimised from 0.492 to $0.466 \mathrm{kWh}$, as shown in Figure 27.

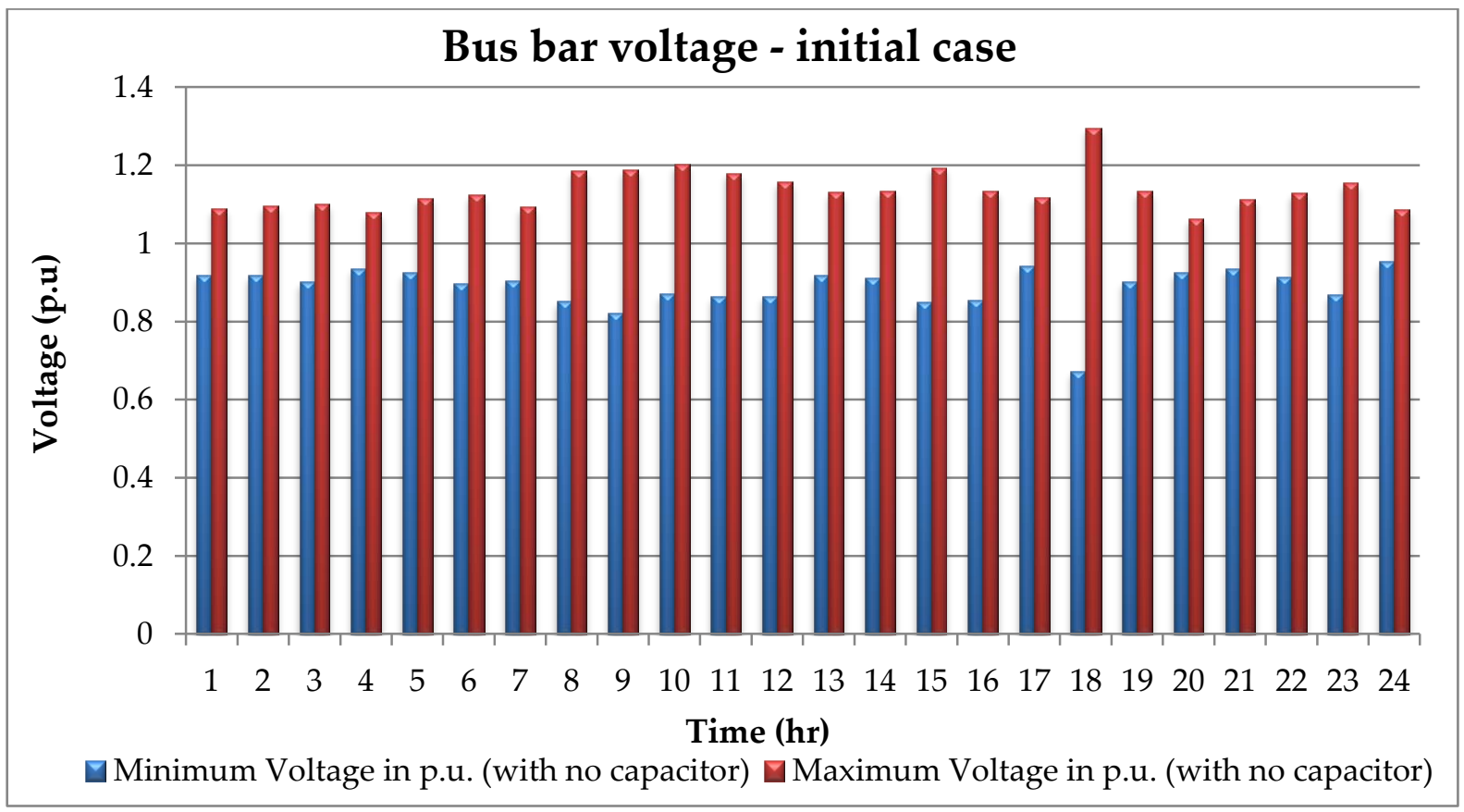

Figure 25: Busbar voltages for initial case 


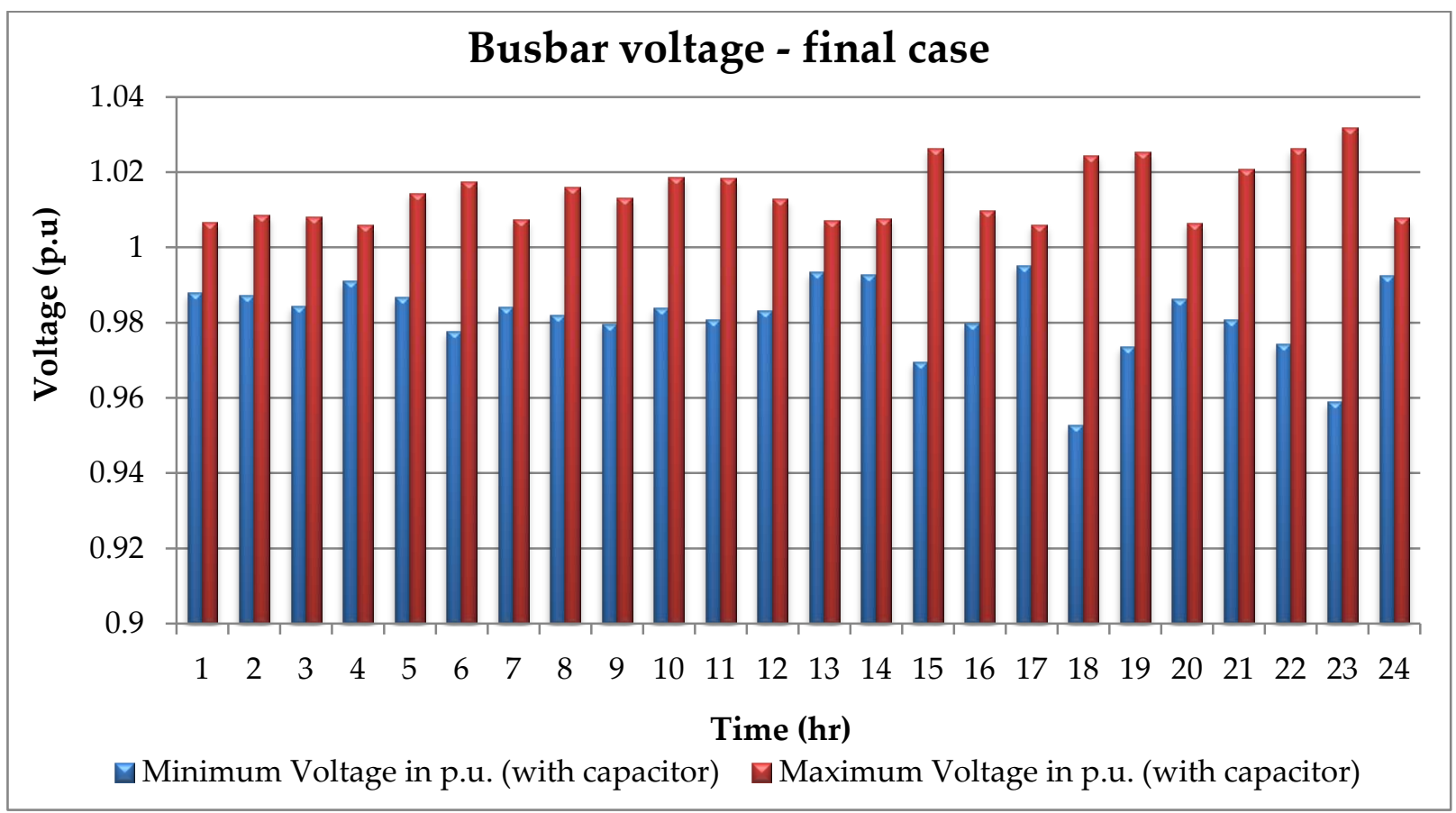

Figure 26: Busbar voltages for final case

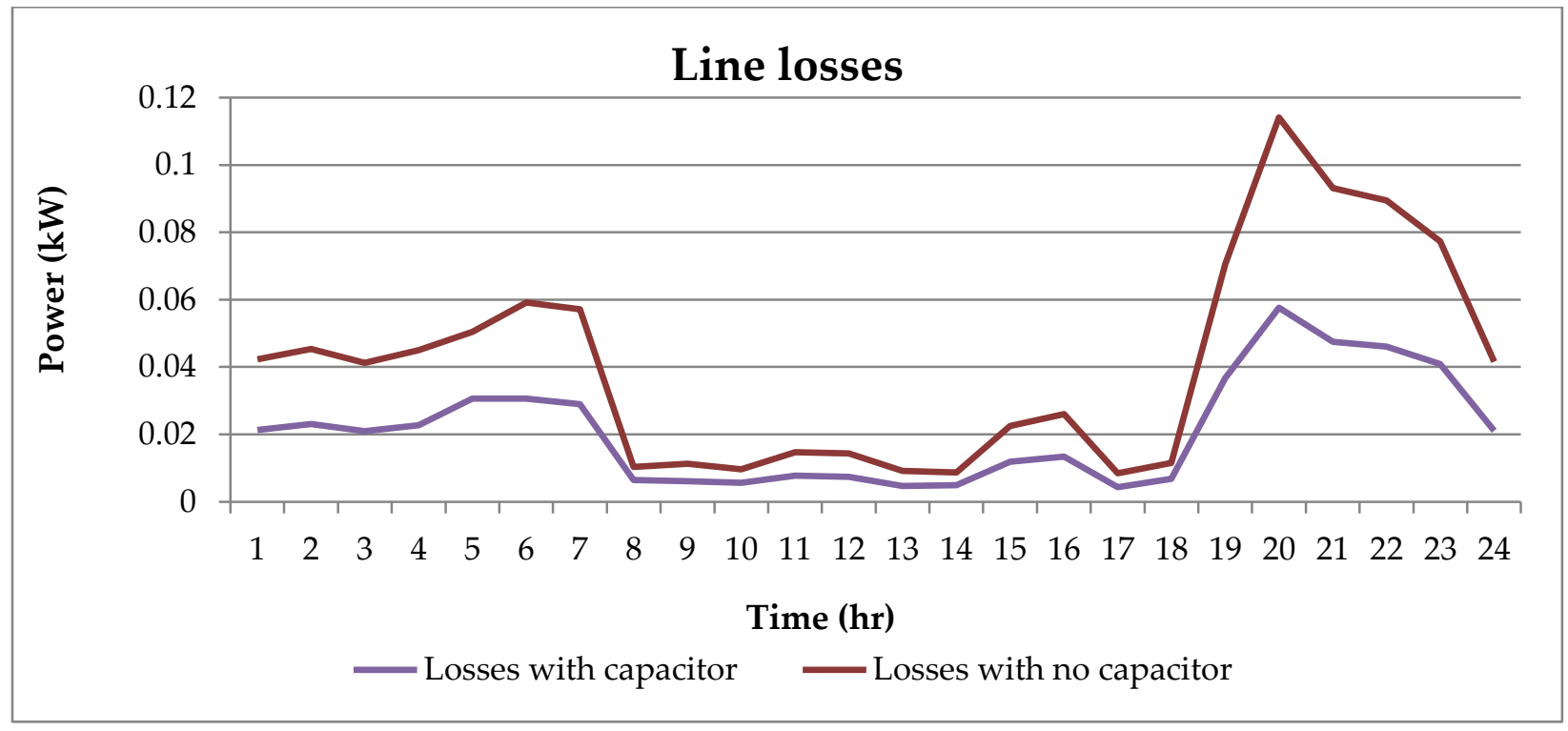

Figure 27: Line losses

2) NW location

The power outputs of the $40.3 \mathrm{~kW}$ PV system for $1^{\text {st }}$ March are shown in Figure 28. The energy produced by the system is $291.13 \mathrm{kWh}, 235.64 \mathrm{kWh}$ and $200.29 \mathrm{kWh}$, based on the PV power (Baseline), PV Power (A. T. L) and PV Power (A. O. L) values, 
respectively. The total losses are $90.84 \mathrm{kWh}$, which is $31.20 \%$ of the baseline value. Also, the L. D. T. T. E and the O. L are $55.49 \mathrm{kWh}$ and $35.35 \mathrm{kWh}$, respectively, and these are $19.06 \%$ and $12.14 \%$ of the baseline value. They are also $61.09 \%$ and $38.91 \%$ of the total losses.

In the afternoon, at $2 \mathrm{p} . \mathrm{m} ., T_{a}$ is $38.1{ }^{\circ} \mathrm{C}$ and it raises the cell temperature, $T_{c}$ to $72.22^{\circ} \mathrm{C}$, at solar irradiance, $G_{a}$ of $1.011 \mathrm{~kW} / \mathrm{m}^{2}$. Also, at $8 a . \mathrm{m}$. $T_{a}$ and $T_{c}$ are $25^{\circ} \mathrm{C}$ and $29.7^{\circ} \mathrm{C}$, respectively at the $G_{a}$ of $0.140 \mathrm{~kW} / \mathrm{m}^{2}$; at 7 p.m. $T_{a}$ and $T_{c}$ are $33.8^{\circ} \mathrm{C}$ and $33.8^{\circ} \mathrm{C}$, respectively, at $G_{a}$ of $0 \mathrm{~kW} / \mathrm{m}^{2}$. These show the daily variation of the locations' irradiance and the ambient temperature. Again, the higher the difference between $T_{a}$ and $T_{\text {stc, }}$ the higher the losses due to temperature effect. An increase in $T_{a}$ decreases $V_{m p p}$, leading to a reduction in the values of $P_{m p p}$ and $\eta$. The energy available to the users on this particular day will, therefore, be $200.29 \mathrm{kWh}$, which can support a demand of $174.98 \mathrm{kWh}$. The minimum and the maximum bus voltage are 0.953 and 1.032p.u, respectively, while the line losses have also been reduced from 0.504 to $0.465 \mathrm{kWh}$.

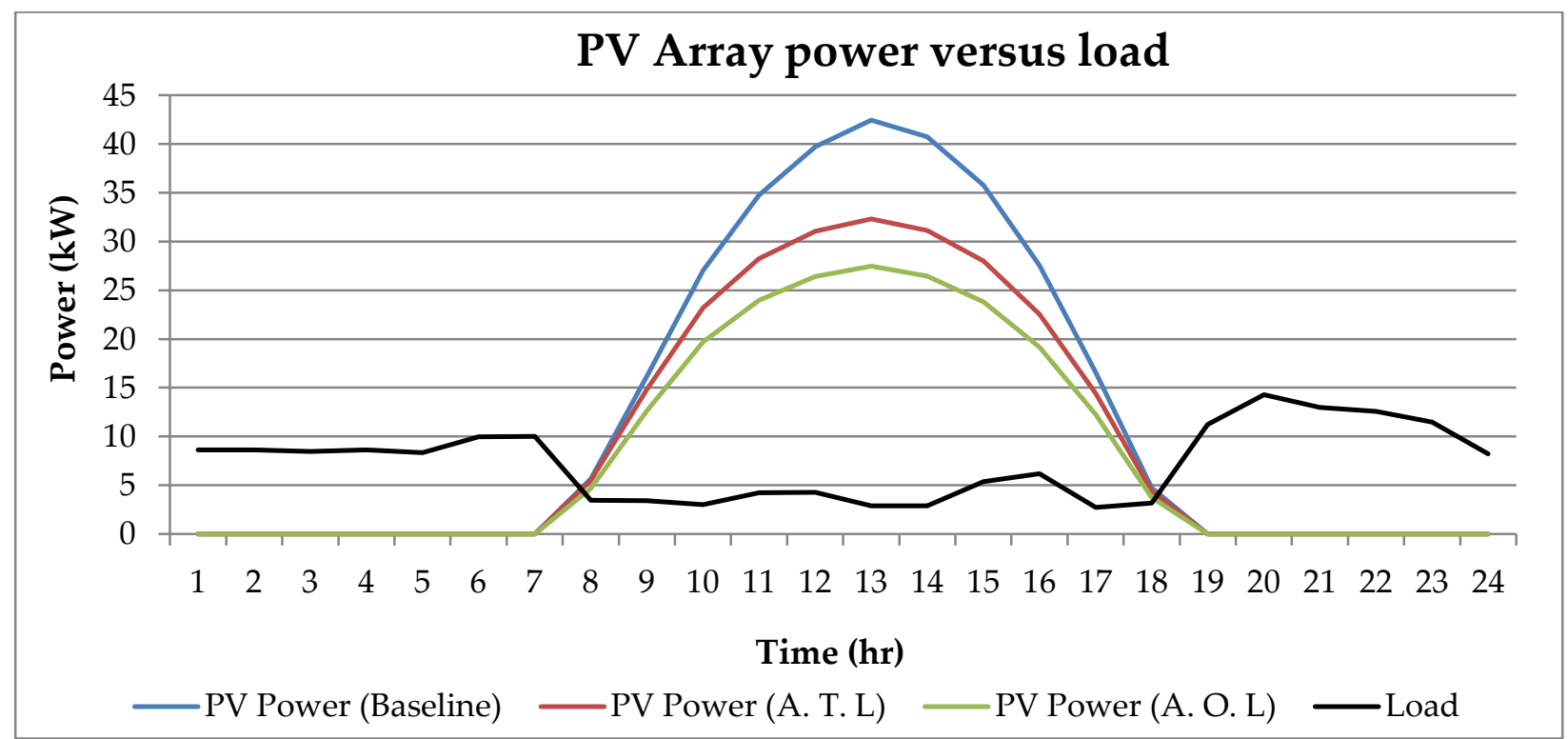

Figure 28: PV array power versus load for the NW zone

3) NC location

The PV system's power outputs for this community are shown in Figure 29. The energy produced is $427.36 \mathrm{kWh}, 339.95 \mathrm{kWh}$ and $288.96 \mathrm{kWh}$, respectively. The total 
losses are $138.40 \mathrm{kWh}$, which is $32.38 \%$ of the baseline value. The L. D. T. T. E and the O. $L$ are $87.41 \mathrm{kWh}$ and $50.99 \mathrm{kWh}$, which are also $20.45 \%$ and $11.93 \%$, respectively, of the baseline value. These losses also translate to $63.16 \%$ and $36.84 \%$ of the total losses.

In the afternoon at $2 \mathrm{p} . \mathrm{m} ., T_{a}$ is $39.3{ }^{\circ} \mathrm{C}$ and it raises the cell temperature, $T_{c}$ to $75.41^{\circ} \mathrm{C}$, at a $G_{a}$ of $1.07 \mathrm{~kW} / \mathrm{m}^{2}$. Also, at 8 a.m. $T_{a}$ and $T_{c}$ are $27.40^{\circ} \mathrm{C}$ and $33.24^{\circ} \mathrm{C}$, respectively, at $G_{a}$ of $0.173 \mathrm{~kW} / \mathrm{m}^{2}$, while at $7 \mathrm{p} . \mathrm{m}$. $T_{a}$ and $T_{c}$ are $36.30^{\circ} \mathrm{C}$ and $36.81^{\circ} \mathrm{C}$, at the $G a$ of $0.015 \mathrm{~kW} / \mathrm{m}^{2}$. These show the daily variation of the locations' irradiance and the ambient temperature. Again, it is found that the higher the difference between $T_{a}$ and $T_{\text {stc }}$, the higher the losses due to the temperature effect. An increase in $T_{a}$ decreases $V_{m p p}$, leading to a reduction in the values of $P_{m p p}$ and $\eta$. The minimum and the maximum busbar voltage are 0.959 and 1.032p.u, respectively. The line losses have also been reduced from 0.502 to $0.464 \mathrm{kWh}$.

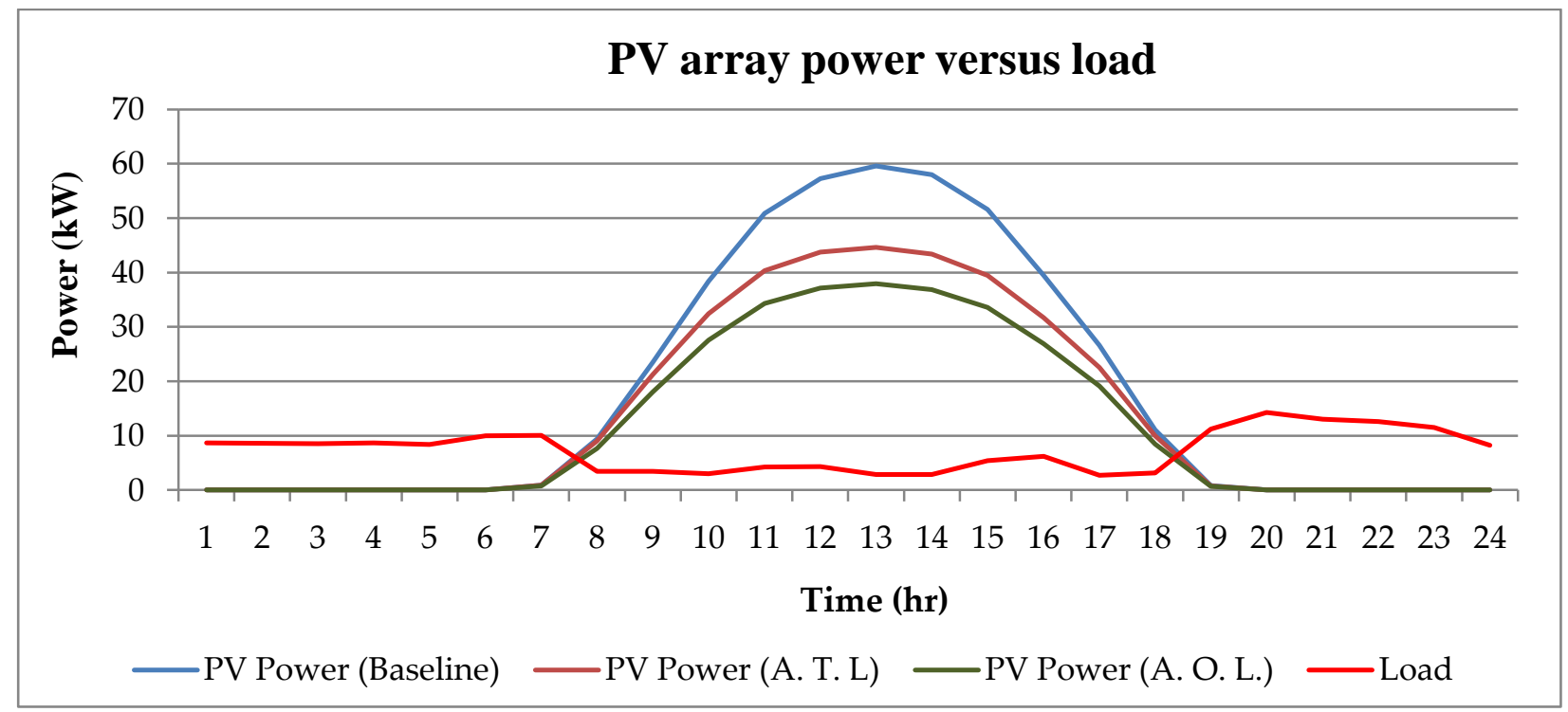

Figure 29: PV array power versus load for the NC zone

4) SE location

The system's power outputs for the community are presented in Figure 30. The energy produced is $364.25 \mathrm{kWh}, 320.93 \mathrm{kWh}$ and $272.79 \mathrm{kWh}$, respectively. The total losses are $91.46 \mathrm{kWh}$, which is $25.11 \%$ of the baseline value. The L. D. T. T. E and the $O$. $L$ are $43.32 \mathrm{kWh}$ and $48.14 \mathrm{kWh}$, respectively, which also translate to $11.92 \%$ and $13.22 \%$ of baseline. The two losses are also $47.36 \%$ and $52.64 \%$, respectively, of the total energy losses. 
In the afternoon, at $2 \mathrm{p} . \mathrm{m} ., T_{a}$ is $28.7^{\circ} \mathrm{C}$ and this raises the cell temperature, $T_{c}$ to $35.40^{\circ} \mathrm{C}$, at a $G_{a}$ of $0.728 \mathrm{~kW} / \mathrm{m}^{2}$. Also, at 8 a.m. $T_{a}$ and $T_{c}$ are $23.50^{\circ} \mathrm{C}$ and $29.78^{\circ} \mathrm{C}$, respectively, at a $G_{a}$ of $0.186 \mathrm{~kW} / \mathrm{m}^{2}$, while at $7 \mathrm{p} . \mathrm{m}$. $T_{a}$ and $T_{c}$ are $27.30^{\circ} \mathrm{C}$ and $27.84^{\circ} \mathrm{C}$, respectively, at a $G_{a}$ of $0.016 \mathrm{~kW} / \mathrm{m}^{2}$. These show the daily variation of the locations' irradiance and the ambient temperature. It is also found that the higher the difference between $T_{a}$ and $T_{\text {stc, }}$ the higher the losses due to temperature effects. The amount of energy available for supplying the $174.98 \mathrm{kWh}$ load demand is $272.79 \mathrm{kWh}$ for that particular day ( $4^{\text {th }}$ April). The minimum and the maximum busbar voltage are 0.953 and 1.031p.u, respectively, while the line losses are being reduced from 0.453 to $0.439 \mathrm{kWh}$.

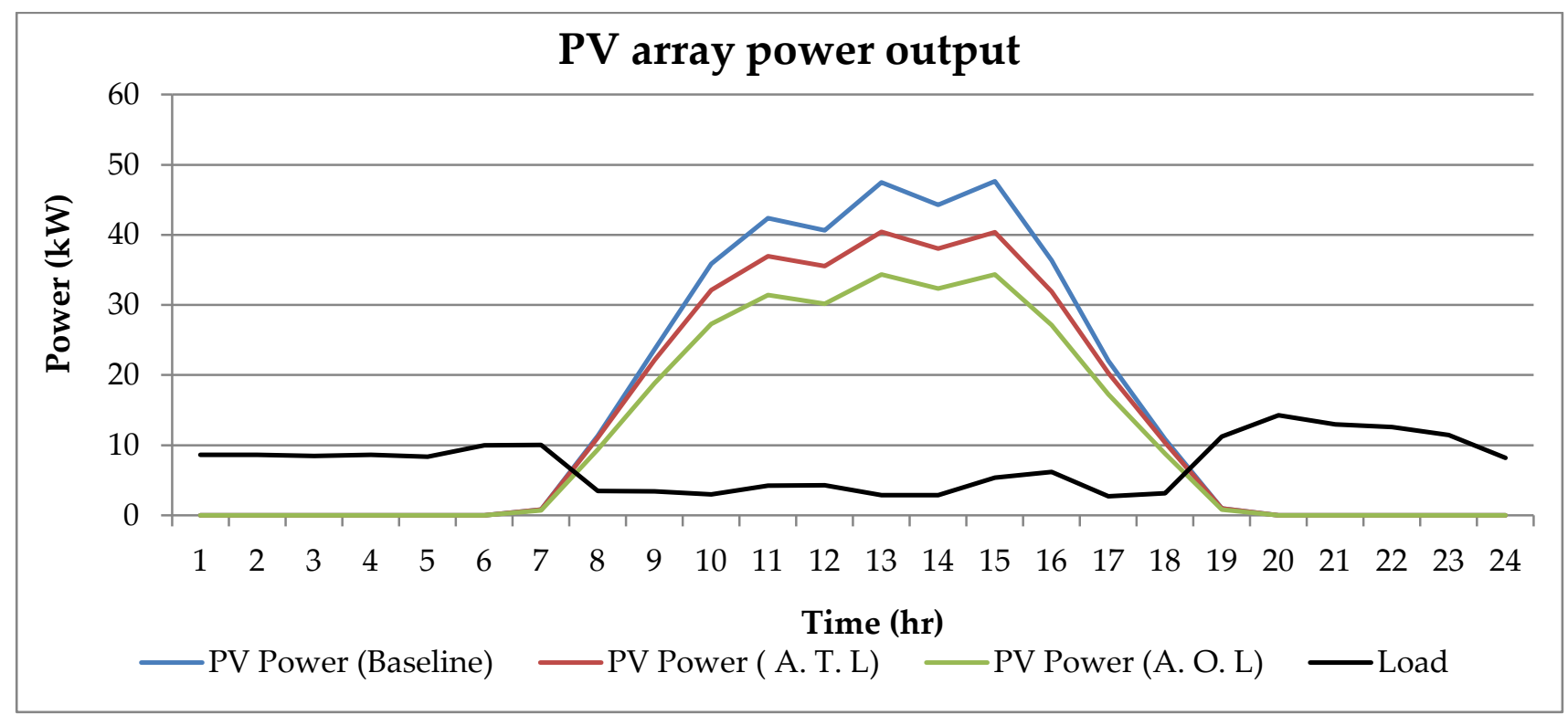

Figure 30: PV array power versus load for the SE zone

5) SS location

The system's power outputs at this location are shown in Figure 31. The energy produced is $252.18 \mathrm{kWh}, 228.95 \mathrm{kWh}$ and $194.61 \mathrm{kWh}$, respectively. The total losses are $57.57 \mathrm{kWh}$, which is $22.83 \%$ of the baseline energy. The L. D. T. T. E and the O. $L$ are $23.23 \mathrm{kWh}$ and $34.34 \mathrm{kWh}$, respectively. These translate to $9.21 \%$ and $13.62 \%$, respectively, of the baseline value; they are also $40.35 \%$ and $59.65 \%$ of the total losses.

In the afternoon at 2 p.m., $T_{a}$ is $32{ }^{\circ} \mathrm{C}$ and this raises $T_{c}$ to $46.80^{\circ} \mathrm{C}$, at a $G_{a}$ of 0.538 $\mathrm{kW} / \mathrm{m}^{2}$. Also, at $8 \mathrm{a} . \mathrm{m} ., T_{a}$ and $T_{c}$ are $22.40^{\circ} \mathrm{C}$ and $26.55^{\circ} \mathrm{C}$, respectively, at a $G_{a}$ of 0.151 
$\mathrm{kW} / \mathrm{m}^{2}$. At 7p.m., $T_{a}$ and $T_{c}$ are $29.30^{\circ} \mathrm{C}$ and $29.33^{\circ} \mathrm{C}$, respectively, at a $G_{a}$ of $0.001 \mathrm{~kW} / \mathrm{m}^{2}$. These reveal the daily variation of the location's solar irradiance and ambient temperature. Again, the results demonstrate that the higher the difference between $T_{a}$ and $T_{\text {stc, }}$ the higher the losses due to temperature effects. The amount of energy that is available for supply the users' demand of $174.98 \mathrm{kWh}$ is, therefore, $194.61 \mathrm{kWh}$ for the specified day (18 $8^{\text {th }}$ April). The minimum and maximum busbar voltages of the model are 0.952 and 1.031p.u, respectively. The line losses are also reduced from 0.467 to 0.440 kWh.

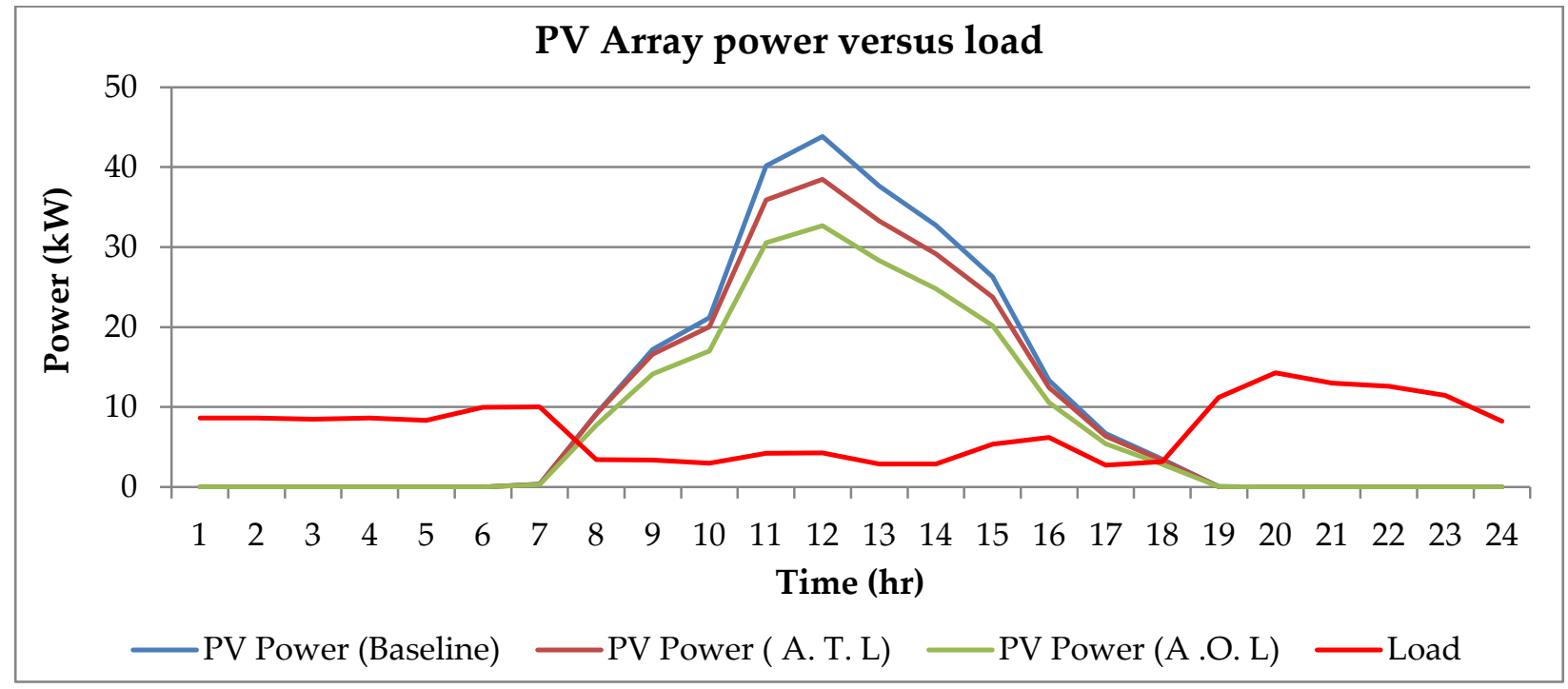

Figure 31: PV array power versus load for the SS zone

6) SW location

The system's power outputs for this location are shown in Figure 32. The energy generated is $329.51 \mathrm{kWh}, 280.09 \mathrm{kWh}$ and $238.07 \mathrm{kWh}$, respectively. The total losses are $91.44 \mathrm{kWh}$, which corresponds to $27.75 \%$ of the baseline value. The L. D. T. T. E and the O. $L$ are $49.42 \mathrm{kWh}$ and $42.02 \mathrm{kWh}$, respectively. These are $15 \%$ and $12.75 \%$ of the baseline value; they are also $54.04 \%$ and $45.96 \%$, respectively of the total losses.

In the afternoon, at $2 \mathrm{p} . \mathrm{m} ., T_{a}$ of $31.3{ }^{\circ} \mathrm{C}$ increases $T_{c}$ to $63.89^{\circ} \mathrm{C}$, at a $G_{a}$ of 1.064 $\mathrm{kW} / \mathrm{m}^{2}$. Also, at $8 \mathrm{p} . \mathrm{m}$., $T_{a}$ of $25.8^{\circ} \mathrm{C}$ increases $T_{c}$ to $31.04^{\circ} \mathrm{C}$, at $G_{a}$ of $0.228 \mathrm{~kW} / \mathrm{m}^{2}$, while at 7p.m., $T_{a}$ of $28.9^{\circ} \mathrm{C}$ leads to a $T_{c}$ of $29.37^{\circ} \mathrm{C}$ at a $G_{a}$ of $0.014 \mathrm{~kW} / \mathrm{m}^{2}$. Again, these demonstrate the daily variation of the location's solar irradiance and ambient temperature. The higher the value of $T_{a}$ minus $T_{s t}$, the higher the losses due to 
temperature effects. The amount of energy available for supplying the users' load demand of $174.98 \mathrm{kWh}$ is $238.07 \mathrm{kWh}$ for the specified day ( $6^{\text {th }}$ of April). The minimum and the maximum busbar voltages are 0.951 and 1.029 p.u, respectively. The line losses have also reduced been reduced from 0.500 to $0.466 \mathrm{kWh}$.

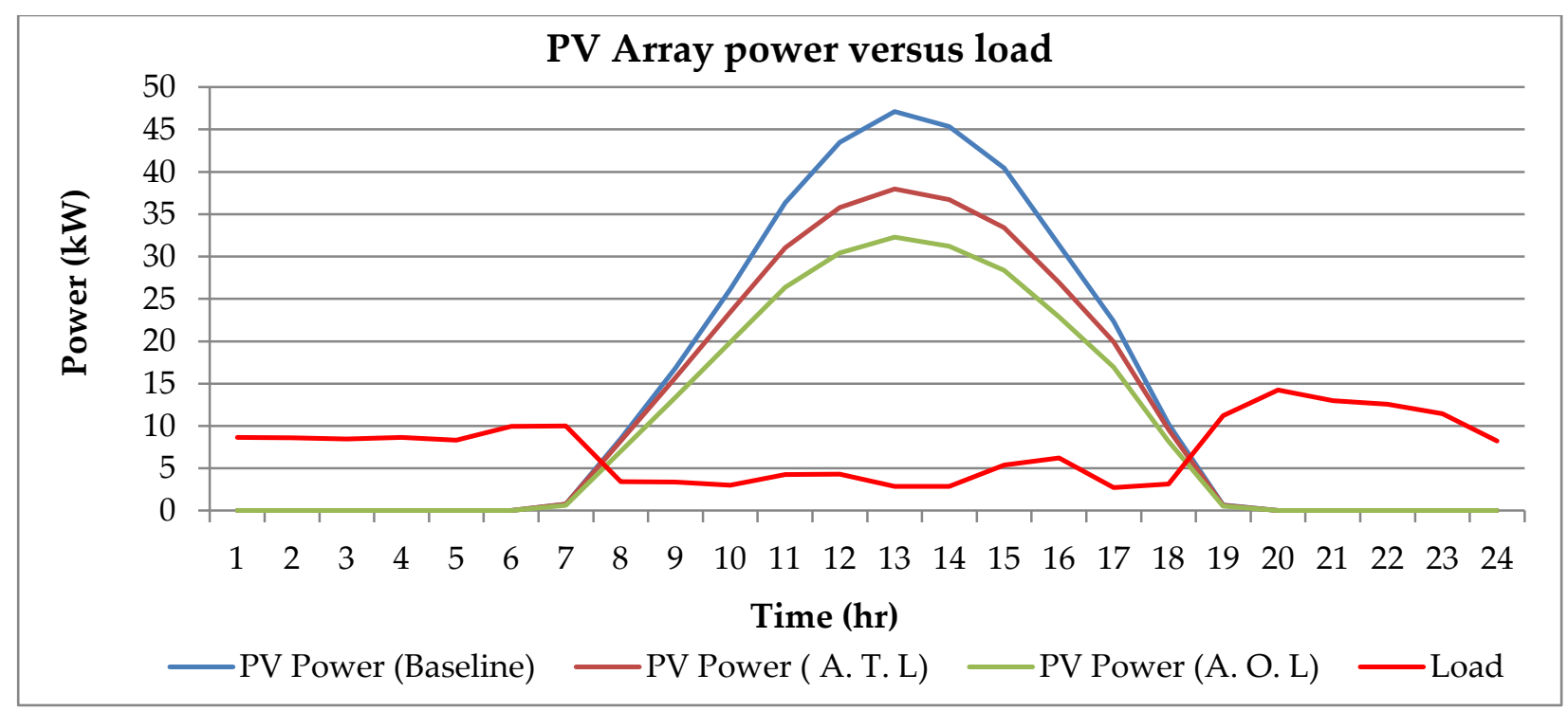

Figure 32: PV array power and the load for the SW zone

It is obvious that there is no much difference in the values of the system's busbar voltages for all the six communities. This is because the same users' load demand and the capacitor rating and settings are used for the simulations. There are also little variations in the values of the line losses. It can be seen that the $10 \mathrm{kVar}$ capacitor is suitable for balancing the voltages for the specified load demand requirement.

There are disparities in the losses due to the locations' different ambient temperature values. The losses due to temperature for the northern region, i.e. NE, NW and NC zones are $20.35 \%, 19.06 \%$ and $20.45 \%$, respectively, of the system's baseline energy value. However, the values obtained for communities in the southern region, i.e. SE, SS and SW zones are less than $20 \%$ of the baseline value. The SE and SS zones have temperature losses of $11.92 \%$ and $9.21 \%$, respectively, of the baseline value, while the SW zone has temperature losses of $15 \%$ of the baseline value. These values are obtained for typical sunny days (i.e. during the dry season) in those locations. The results show that $T_{c}=T_{\text {stc }}$ when $T_{a}=T_{\text {stc }}$ at $G_{a}=0$, but $T_{c}>T_{\text {stc }}$ when $T_{a}>T_{s t c}$ at $G_{a}>0$. 
Furthermore, the results reveal that the northern zones have relatively lower values for the other losses (O.L), compared to the southern zones. The NE, NW and NC zones have $O$. Ls of $11.95 \%, 12.14 \%$ and $11.93 \%$, respectively, of the baseline output. However, the values obtained for the SE, SS and SW zones are $13.22 \%, 13.62 \%$ and $12.75 \%$, respectively. Such losses are possible because the southern zones have a lower incomplete utilisation of the solar irradiation than do the northern zones. The results further show that NE, NW, NC, SE, SS and SW have total losses of 32.3\%, 31.2\%, $32.38 \%, 25.11 \%, 22.83 \%$ and $27.75 \%$, respectively, of the corresponding baseline values.

Furthermore, there are differences in the amounts of energy that are produced on a daily basis in those locations. This is because of the disparities in solar energy potential for the locations and the intermittent nature of the energy source. The results demonstrate that the NE zone has the highest solar energy potential in the northern region followed by the NW and the NC zones. Similarly, the SW zone has the highest solar energy potential in the southern region followed by the SE and the SS zones.

The performance analysis results also show that the systems' energy outputs for the selected days are enough to support the users' load demand of $174.98 \mathrm{kWh}$, while the excess energy outputs are expected to be stored in the battery bank. However, this may not be so during the rainy season where there is a relatively lower irradiation cycle. In this case, the available energy may not be enough to support the specified users' load demand requirements. Therefore, this thesis focuses on analysing the SPM's performance for all the seasons in the year (i.e. both dry and rainy seasons). This will be presented in the later part of this chapter.

\subsubsection{Yearly power, energy and efficiency}

The analysis of the power outputs, energy yield and the efficiency of the different photovoltaic systems are based on the IEC 61724 standard. Such an analysis concentrates on evaluating the system performance for 12 hours of the day (i.e. when the sun is available). The PV array power outputs, power losses, efficiency, energy yield and the losses of the different system capacities are shown in Figures 33 to 39.

The array yield and losses are usually expressed in normalised values (i.e. $\mathrm{kWh} / \mathrm{kW}$ ) in order to provide a "performance summary" that is appropriate for 
comparing the SPMs with different photovoltaic system configurations or designs operating in different locations [136].

The normalised indices such as yields, losses and the efficiencies are crucial in comparing the performance of different systems. The array yield expressed in $\mathrm{kWh} / \mathrm{kW}$ represents the time during which the PV energy system needs to operate at its rated power capacity, so as to produce the same amount of energy that it actually produces. This may be expressed over a day, thus, having a unit of hr/d or over a year with a unit of $\mathrm{hr} / \mathrm{yr}$. The array losses, on the other hand, expressed in $\mathrm{kWh} / \mathrm{kW}$ represent the duration that the PV system needs to operate at its rated capacity in order to compensate for the energy losses. These are also measured in $\mathrm{hr} / \mathrm{d}$ or $\mathrm{hr} / \mathrm{yr}$.

\section{1) NE location}

The power output of the $40.07 \mathrm{~kW}$ PV system in the NE location is shown in Figures 33 to 35, based on the baseline, A. T. $L$ and A. O. $L$ values. The system has the highest power output of $22.29 \mathrm{~kW}$ in April, compared to the other months. The lowest power output is obtained in August, which is $17.50 \mathrm{~kW}$. The power outputs after considering the temperature losses $(A . T$. L) for these two months are $19.73 \mathrm{~kW}$ and $16.27 \mathrm{~kW}$, respectively. The total losses are in April are $5.51 \mathrm{~kW}$ (i.e. $24.72 \%$ of baseline power), while the value obtained in August is $3.67 \mathrm{~kW}$ (i.e. $20.97 \%$ of baseline power), as shown in Figure 36.

The L. D. T. T. E for April and August are $2.554 \mathrm{~kW}$ and $1.23 \mathrm{~kW}$, respectively. The system's power outputs in theses months after considering the other losses (A. O. L) are $16.77 \mathrm{~kW}$ and $13.83 \mathrm{~kW}$, respectively. Therefore, the corresponding $O$. Ls are 2.96 $\mathrm{kW}$ and $2.44 \mathrm{~kW}$. It is obvious from these results that the proposed SPM has higher total losses (i.e. L. D. T. T. E plus O. L) in April, compared to August. This is due to both the seasonal variation and the difference in the solar energy capture. The results shown in Figure 37 indicate that the system's efficiency is higher in August than in April and other months (i.e. $13.85 \%$ versus $13.19 \%$ ).

The $40.07 \mathrm{~kW}$ SPM's yearly energy production is shown in Figure 38. The baseline, A. T. $L$ and A. O. $L$ values are $89,129 \mathrm{kWh}, 81,213 \mathrm{kWh}$ and $69,031 \mathrm{kWh}$, respectively. Also, the yearly energy yields are 2,224 kWh/kW, 2,027 kWh/kW and 1,723 $\mathrm{kWh} / \mathrm{kW}$, respectively (see Figure 39). These values translate to the corresponding 
values of $6.09 \mathrm{hr} / \mathrm{d}, 5.55 \mathrm{hr} / \mathrm{d}$ and $4.72 \mathrm{hr} / \mathrm{d}$, on a daily basis. The yearly values of the $L$. D. T. T. E and O. LS are $197.54 \mathrm{kWh} / \mathrm{kW}$ and $304.02 \mathrm{kWh} / \mathrm{kW}$, respectively, which are also expressed $0.54 \mathrm{hr} / \mathrm{d}$ and $0.83 \mathrm{hr} / \mathrm{d}$, respectively. The total losses are $501.56 \mathrm{kWh} / \mathrm{kW}$, as shown in Figure 40, and are also expressed as $1.37 \mathrm{hr} / \mathrm{d}$. The total SPM losses are $22.55 \%$ of the baseline value.

\section{2) NW location}

As shown in Figures 33 to 35, the $40.3 \mathrm{~kW}$ SPM has the highest baseline power output of $22.85 \mathrm{~kW}$ in March, compared to the other months. The lowest baseline power output is obtained in August, which is $17.49 \mathrm{~kW}$. The A. T. Ls for the months are 20.22 $\mathrm{kW}$ and $16.09 \mathrm{~kW}$, respectively. The corresponding L. D. T. T. E values are $2.63 \mathrm{~kW}$ and $1.4 \mathrm{~kW}$, respectively. Also, the $A$. $O$. Ls are $17.19 \mathrm{~kW}$ and $13.68 \mathrm{~kW}$, respectively. The total losses are $5.66 \mathrm{~kW}$ (i.e. $24.77 \%$ of baseline power) and $3.82 \mathrm{~kW}$ (i.e. $21.84 \%$ of baseline power), respectively, for the months as shown in Figure 36. Again, the results demonstrate that the total losses obtained for March are higher than that of August. In addition, the month of August has a higher average efficiency compared to April (i.e. $13.70 \%$ versus $13.12 \%$ ), as presented in Figure 37.

The SPM's yearly energy production is shown in Figure 38. The values reported are $87,250 \mathrm{kWh}, 79,007 \mathrm{kWh}$ and $67,156 \mathrm{kWh}$, respectively. Also, its yearly energy yields are 2,165 kWh/kW, 1,960 kWh/kW and 1,666 kWh/kW, respectively, as shown in Figure 39. These values are also expressed as $5.93 \mathrm{hr} / \mathrm{d}, 5.37 \mathrm{hr} / \mathrm{d}$ and $4.57 \mathrm{hr} / \mathrm{d}$. The corresponding L. D. T. T. E and the O. Ls are $204.55 \mathrm{kWh} / \mathrm{kW}$ (i.e. $9.45 \%$ of baseline yield) and $294.07 \mathrm{kWh} / \mathrm{kW}$ (i.e. 13.58\% of baseline yield), as shown in Figure 40. These translate to daily performance indices of $0.56 \mathrm{hr} / \mathrm{d}$ and $0.81 \mathrm{hr} / \mathrm{d}$, respectively. The total losses are $498.62 \mathrm{kWh} / \mathrm{kW}$ (i.e. $1.37 \mathrm{hr} / \mathrm{d}$ ), which is $23.06 \%$ of the baseline yield.

\section{3) NC location}

The $54.15 \mathrm{~kW}$ SPM's power outputs are shown in Figures 33 to 35. It can be seen that the system has the highest baseline power output of $29.86 \mathrm{~kW}$ in February. The least baseline output of $17.31 \mathrm{~kW}$ is obtained in July. The $A$. T. Ls for these months are $24.78 \mathrm{~kW}$ and $15.85 \mathrm{~kW}$, respectively, while the corresponding $A$. O. Ls are $21.07 \mathrm{~kW}$ and $13.47 \mathrm{~kW}$. The total losses are $8.80 \mathrm{~kW}$ and $3.84 \mathrm{~kW}$, which are $29.47 \%$ and $22.18 \%$ of the corresponding baseline values (see Figure 36). The L. D. T. T. Es are $5.08 \mathrm{~kW}$ and 
$1.46 \mathrm{~kW}$, while the O.Ls are $3.72 \mathrm{~kW}$ and $2.38 \mathrm{~kW}$, respectively. Again, the results reveal that the L. D. T. T. E and O.Ls are higher in February than in July. This indicates that the total losses are higher in February than in July and the other months. The SPM's efficiency in February and July is $12.4 \%$ and $13.6 \%$, respectively, as shown in Figure 37.

The SPM's yearly energy production is shown in Figure 38, with the values of 106,605 kWh, 92, $885 \mathrm{kWh}$ and 78,952 kWh. The yearly yields are presented in Figure 39 and they are $1,969 \mathrm{kWh} / \mathrm{kW}, 1,715 \mathrm{kWh} / \mathrm{kW}$ and $1,458 \mathrm{kWh} / \mathrm{kW}$, respectively. These values are also interpreted as $5.39 \mathrm{hr} / \mathrm{d}, 4.70 \mathrm{hr} / \mathrm{d}$ and $3.99 \mathrm{hr} / \mathrm{d}$. The L. D. T. T. E and the O. Ls are $253.37 \mathrm{kWh} / \mathrm{kW}$ and $257.30 \mathrm{kWh} / \mathrm{kW}$, respectively, which are also expressed as $0.69 \mathrm{hr} / \mathrm{d}$ and $0.70 \mathrm{hr} / \mathrm{d}$. The total losses are $510.67 \mathrm{kWh} / \mathrm{kW}$ (i.e. $1.40 \mathrm{hr} / \mathrm{d}$ ); this value is $25.94 \%$ of the baseline value. It can be observed that the total SPM's losses obtained for Gwagwalada are higher than those of Bununu and Gusau (see Figure 40).

\section{4) SE location}

The $60.86 \mathrm{~kW}$ SPM's power outputs for this location are shown in Figures 33 to 35. The highest baseline power output of $28.58 \mathrm{~kW}$ is obtained in March, while the least value of $17.49 \mathrm{~kW}$ is obtained in August. The A. T. Ls are $25.93 \mathrm{~kW}$ and $16.67 \mathrm{~kW}$ respectively, while the corresponding $A$. $O$. Ls are $22.04 \mathrm{~kW}$ and $14.17 \mathrm{~kW}$. The total losses obtained for the two months are $6.53 \mathrm{~kW}$ and $3.32 \mathrm{~kW}$, respectively. These values are $22.85 \%$ and $18.98 \%$, respectively, of the corresponding baseline values.

The L. D. T. T. Es for the months are $2.64 \mathrm{~kW}$ and $0.82 \mathrm{~kW}$, while the values of the $O$. Ls are $3.89 \mathrm{~kW}$ and $2.50 \mathrm{~kW}$, respectively. These results show that the SPM has higher total losses in March than in August, as shown in Figure 36. The SPM is most efficient in August (see Figure 37). It has an efficiency of $14.20 \%$ and $13.52 \%$ in August and March, respectively.

The system's yearly energy production is shown in Figure 38, with the values of $108,699 \mathrm{kWh}, 100,483 \mathrm{kWh}$ and 85,410 $\mathrm{kWh}$. The yearly yields are expressed as 1,786 $\mathrm{kWh} / \mathrm{kW}, 1,651 \mathrm{kWh} / \mathrm{kW}$ and 1,403 kWh/kW, respectively (shown in Figure 39). These values are also expressed as $4.89 \mathrm{hr} / \mathrm{d}, 4.52 \mathrm{hr} / \mathrm{d}$ and $3.84 \mathrm{hr} / \mathrm{d}$, respectively. The total losses are $382.66 \mathrm{kWh} / \mathrm{kW}$ (i.e. $1.05 \mathrm{hr} / \mathrm{d}$ ), whose value is $21.42 \%$ of the baseline yield. The L. D. T. T. E and the O. Ls are $135 \mathrm{kWh} / \mathrm{kW}$ and $247.66 \mathrm{kWh} / \mathrm{kW}$, which also translated to $0.37 \mathrm{hr} / \mathrm{d}$ and $0.68 \mathrm{hr} / \mathrm{d}$, respectively (see Figure 40). This analysis 
demonstrates that the SPM's yield and total losses obtained for SE location are lower than those obtained for the NE, NW and NC zones. The system is also found to have a higher efficiency than the three communities in the northern region earlier discussed.

5) SS location

The $60.87 \mathrm{~kW}$ SPM's power outputs are presented in Figures 33 to 35. The highest baseline power output of $23.50 \mathrm{~kW}$ is obtained in April, while the lowest value of 17.55 $\mathrm{kW}$ is obtained in July. The A. T. Ls are $21.72 \mathrm{~kW}$ and $16.65 \mathrm{~kW}$, while the $A$. O. Ls are $18.47 \mathrm{~kW}$ and $14.15 \mathrm{~kW}$, respectively. Also, the total losses obtained for the two months are $5.04 \mathrm{~kW}$ and $3.40 \mathrm{~kW}$, respectively (i.e. $21.45 \%$ and $19.37 \%$ of the corresponding baseline values). The L. D. T. T. Es are $1.78 \mathrm{~kW}$ and $0.90 \mathrm{~kW}$, while the $O . L s$ are $3.26 \mathrm{~kW}$ and $2.50 \mathrm{~kW}$, respectively. The total losses are higher in April compared to July (shown in Figure 36). The energy system is most efficient in July (see Figure 37). It has an efficiency of $13.77 \%$ and $14.14 \%$ in March and July, respectively.

The system's yearly energy production is shown in Figure 38, with the values of 90,939 $\mathrm{kWh}, 85,085 \mathrm{kWh}$ and 72,322 $\mathrm{kWh}$. The yearly energy yields are 1,494 kWh/kW, 1,398 kWh/kW and 1,188 kWh/kW, respectively (see Figure 39), which are $4.09 \mathrm{hr} / \mathrm{d}, 3.83$ $\mathrm{hr} / \mathrm{d}$ and $3.26 \mathrm{hr} / \mathrm{d}$, respectively. The total losses are $305.84 \mathrm{kWh} / \mathrm{kW}$ (i.e. $0.84 \mathrm{hr} / \mathrm{d}$ ) and this is $20.47 \%$ of the baseline value. The L. D. T. T. E and the O. Ls are $96.17 \mathrm{kWh} / \mathrm{kW}$ and $209.67 \mathrm{kWh} / \mathrm{kW}$ (see Figure 40); these are also expressed as $0.26 \mathrm{hr} / \mathrm{d}$ and $0.58 \mathrm{hr} / \mathrm{d}$. The SPM's yields and losses obtained for SS location are lower than those obtained for the NE, NW, NC and SE locations, though it has the lowest solar energy potential of all the zones. The performance result also demonstrates that the system has a higher efficiency than those systems in the four locations earlier discussed.

6) SW location

The $46.66 \mathrm{~kW}$ SPM's power outputs are shown in Figures 33 to 35. The highest baseline power output of $23.24 \mathrm{~kW}$ is obtained in April, while the least value of 17.95 $\mathrm{kW}$ is obtained in June. The $A$. T. Ls are $21.03 \mathrm{~kW}$ and $16.76 \mathrm{~kW}$, while the $A$. $O$. Ls are $17.87 \mathrm{~kW}$ and $14.25 \mathrm{~kW}$, respectively, for the two months. In addition, the total losses are $5.37 \mathrm{~kW}$ and $3.70 \mathrm{~kW}$, which correspond to $23.11 \%$ and $20.61 \%$ of the baseline values (see Figure 36). The results indicate that the system is most efficient in July, with an efficiency of $13.91 \%$, compared to a value of $13.42 \%$ obtained for April. 
The system's yearly energy production is shown in Figure 38, with values of $88,964 \mathrm{kWh}, 81,803 \mathrm{kWh}$ and 69,532 kWh. The yearly energy yields are 1,907 kWh/kW, $1,753 \mathrm{kWh} / \mathrm{kW}$ and $1,490 \mathrm{kWh} / \mathrm{kW}$, respectively (see Figure 39). These are expressed as $5.22 \mathrm{hr} / \mathrm{d}, 4.80 \mathrm{hr} / \mathrm{d}$ and $4.08 \mathrm{hr} / \mathrm{d}$. The total losses are $416.46 \mathrm{kWh} / \mathrm{kW}$ (i.e. $1.14 \mathrm{hr} / \mathrm{d}$ ), which also translates to $21.84 \%$ of the baseline value. The L. D. T. T. E and O. Ls are $153.48 \mathrm{kWh} / \mathrm{kW}$ and $262.97 \mathrm{kWh} / \mathrm{kW}$, and they are expressed as $0.42 \mathrm{hr} / \mathrm{d}$ and $0.72 \mathrm{hr} / \mathrm{d}$, respectively (see Figure 40). The results show that the system's yields and losses are higher than those obtained for the two other locations within the southern region - SE and SS zones. However, the SPM system in Lisa has lower yields and losses compared to those in the northern region - NE, NW and NC locations.

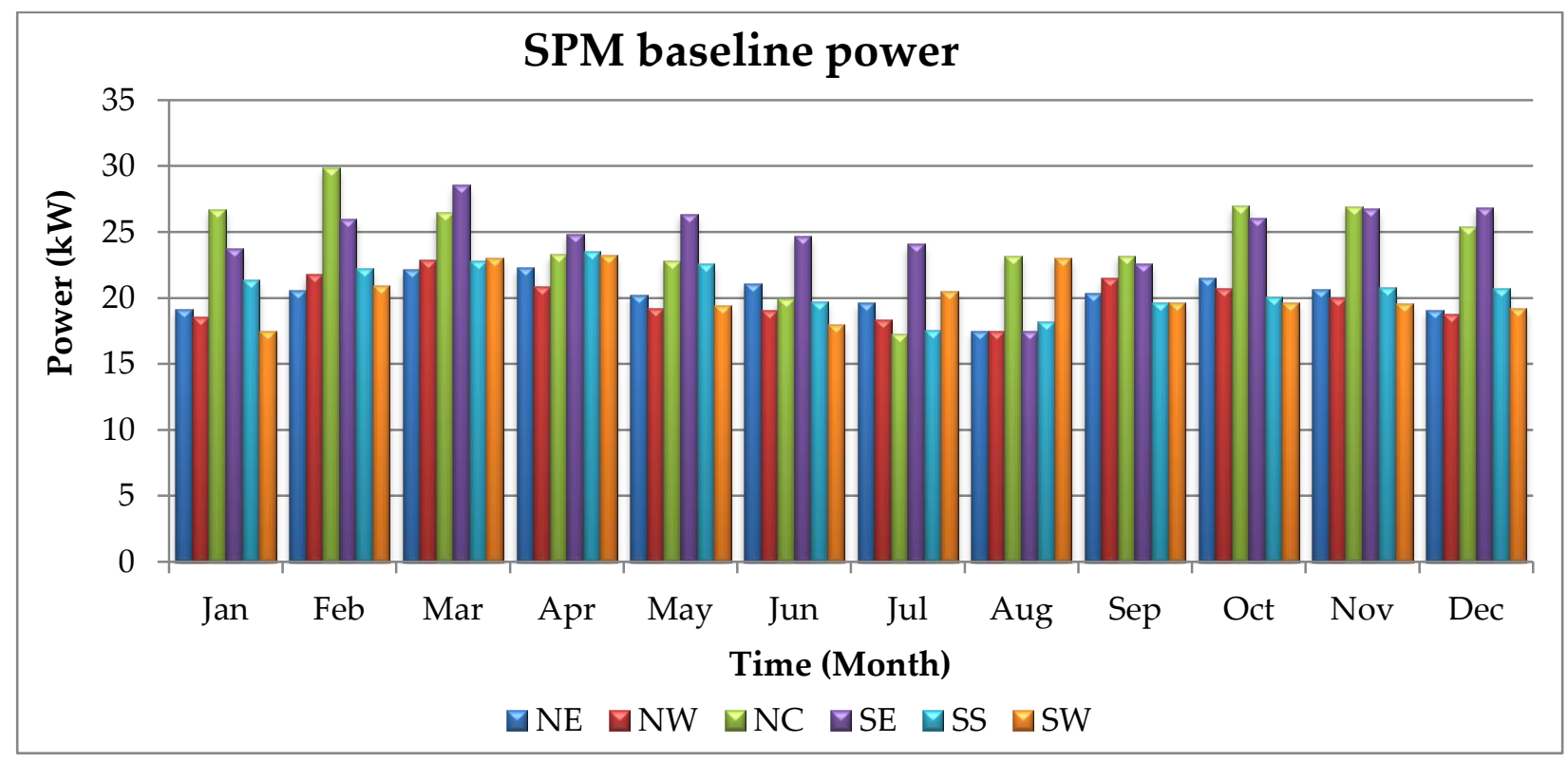

Figure 33: SPM systems' baseline power outputs 


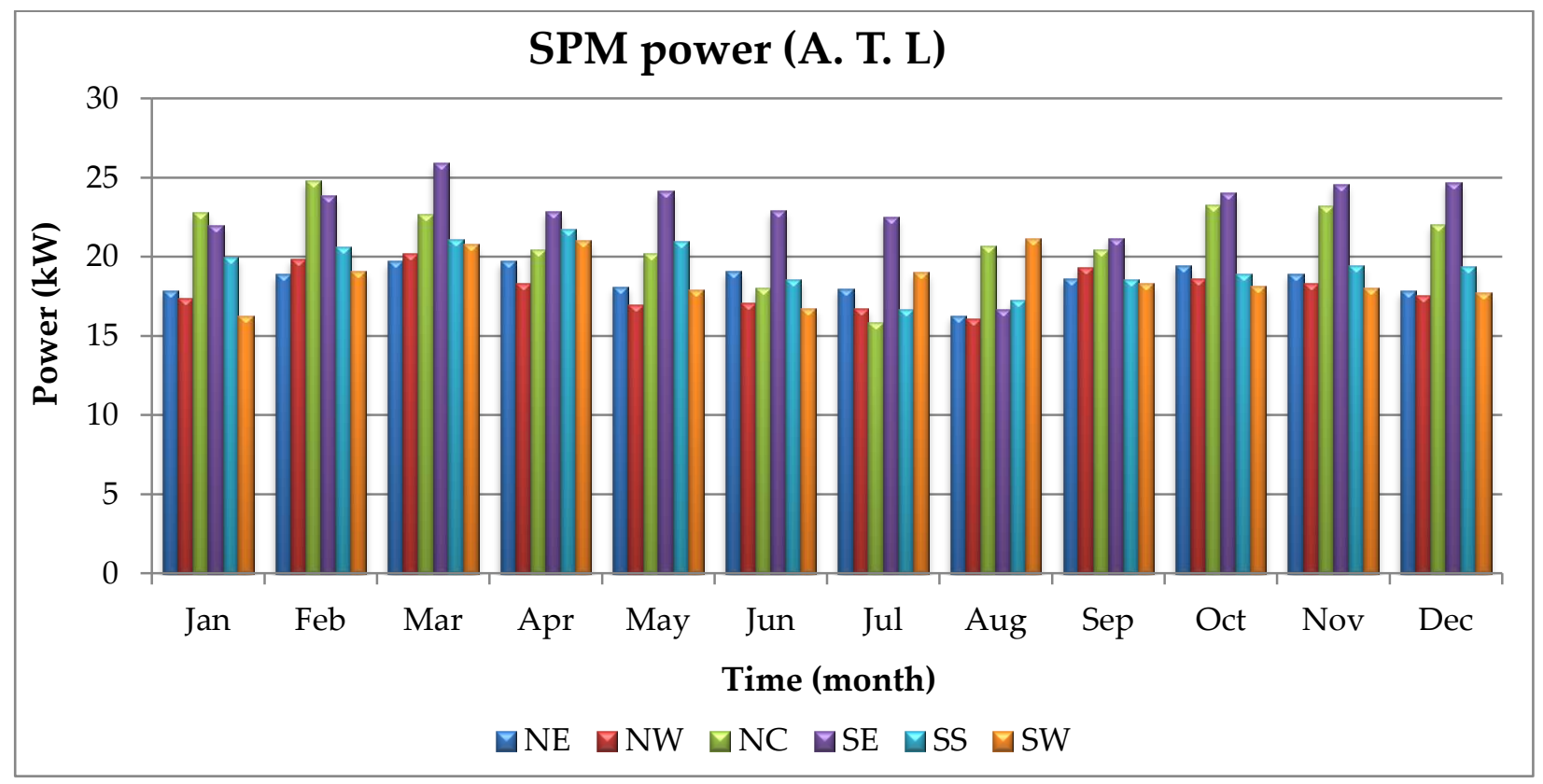

Figure 34: SPM systems' power outputs after temperature losses

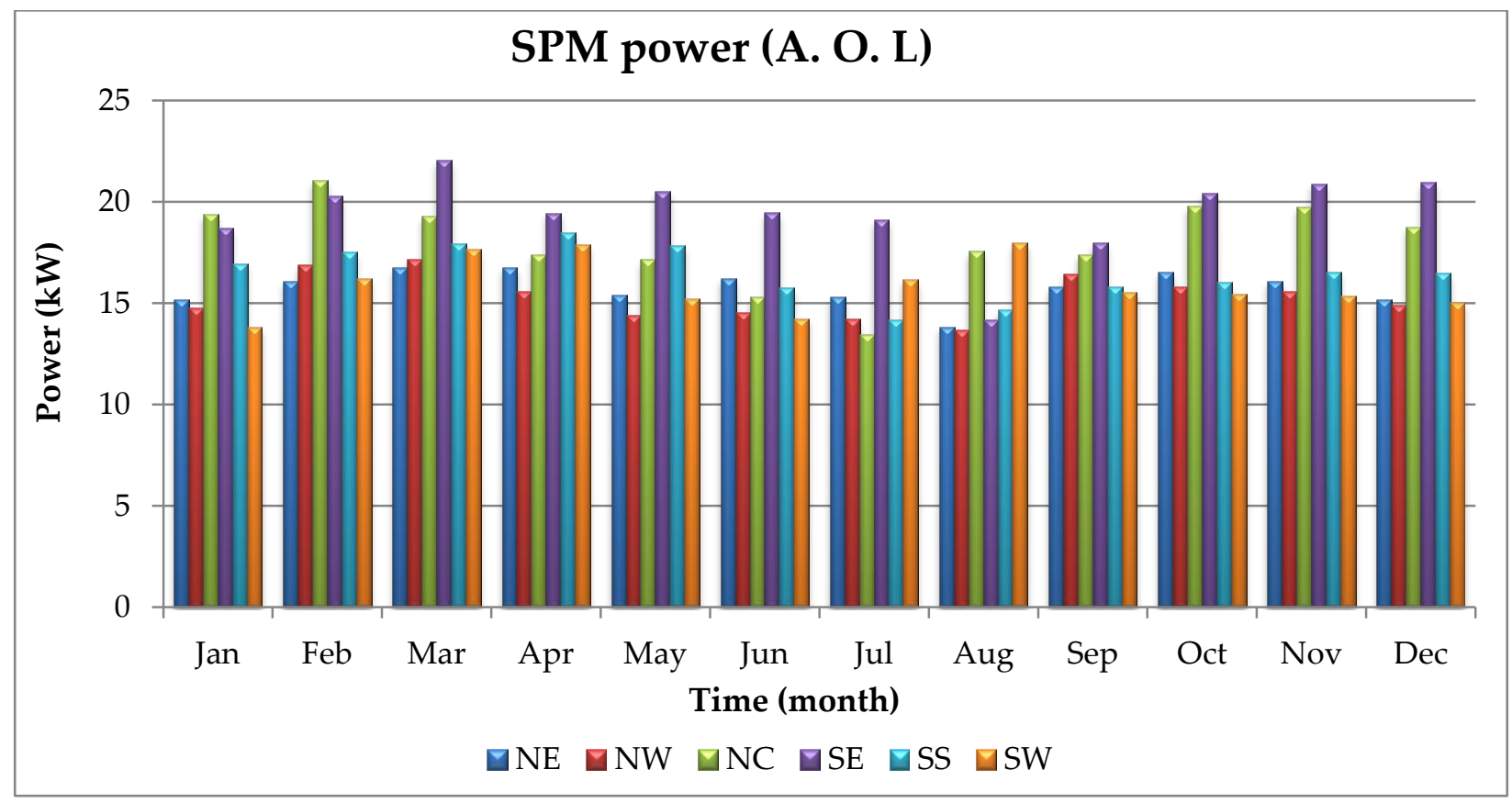

Figure 35: SPM systems' power outputs after temperature and other losses 


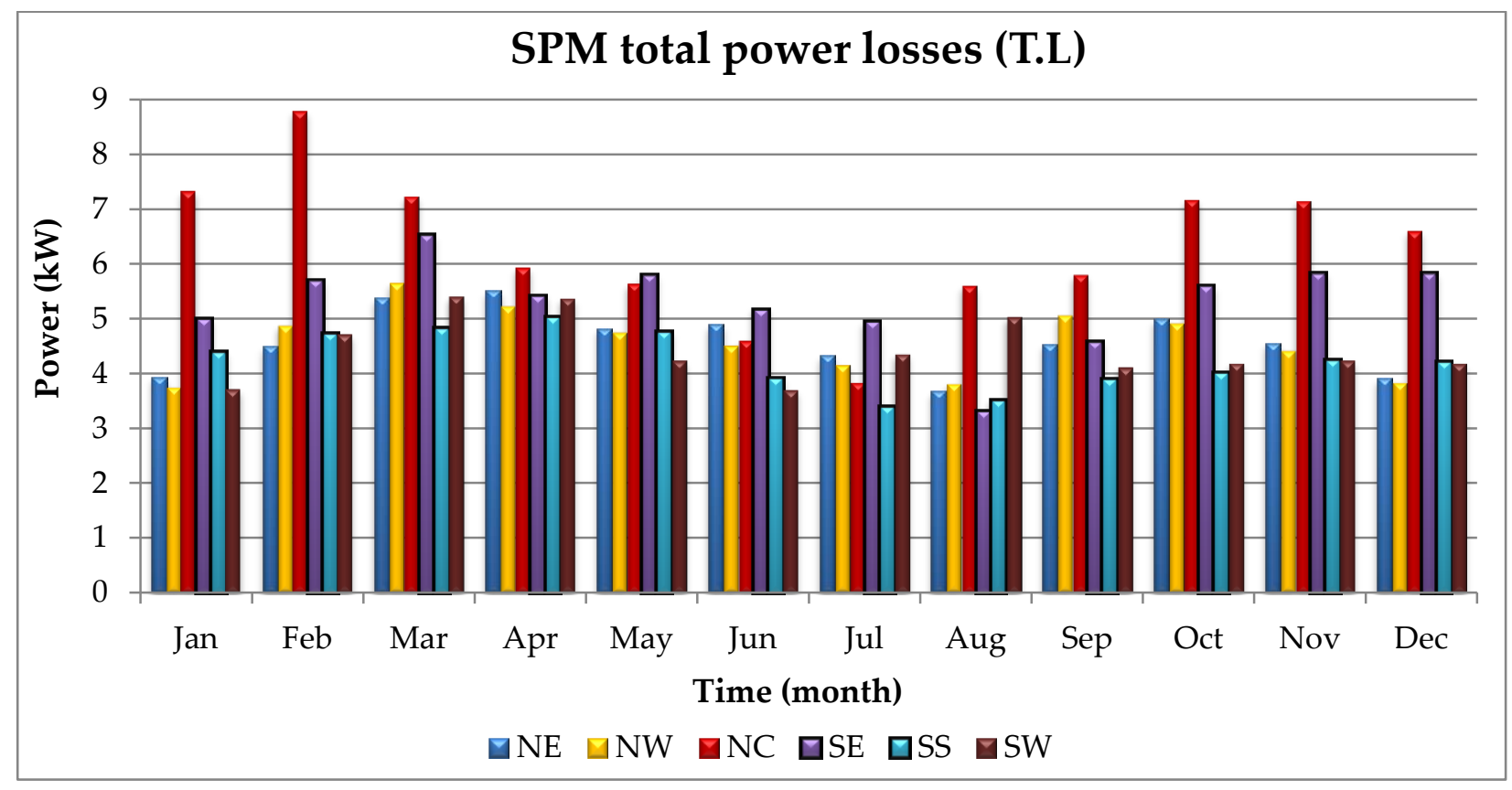

Figure 36: SPM systems' total power losses

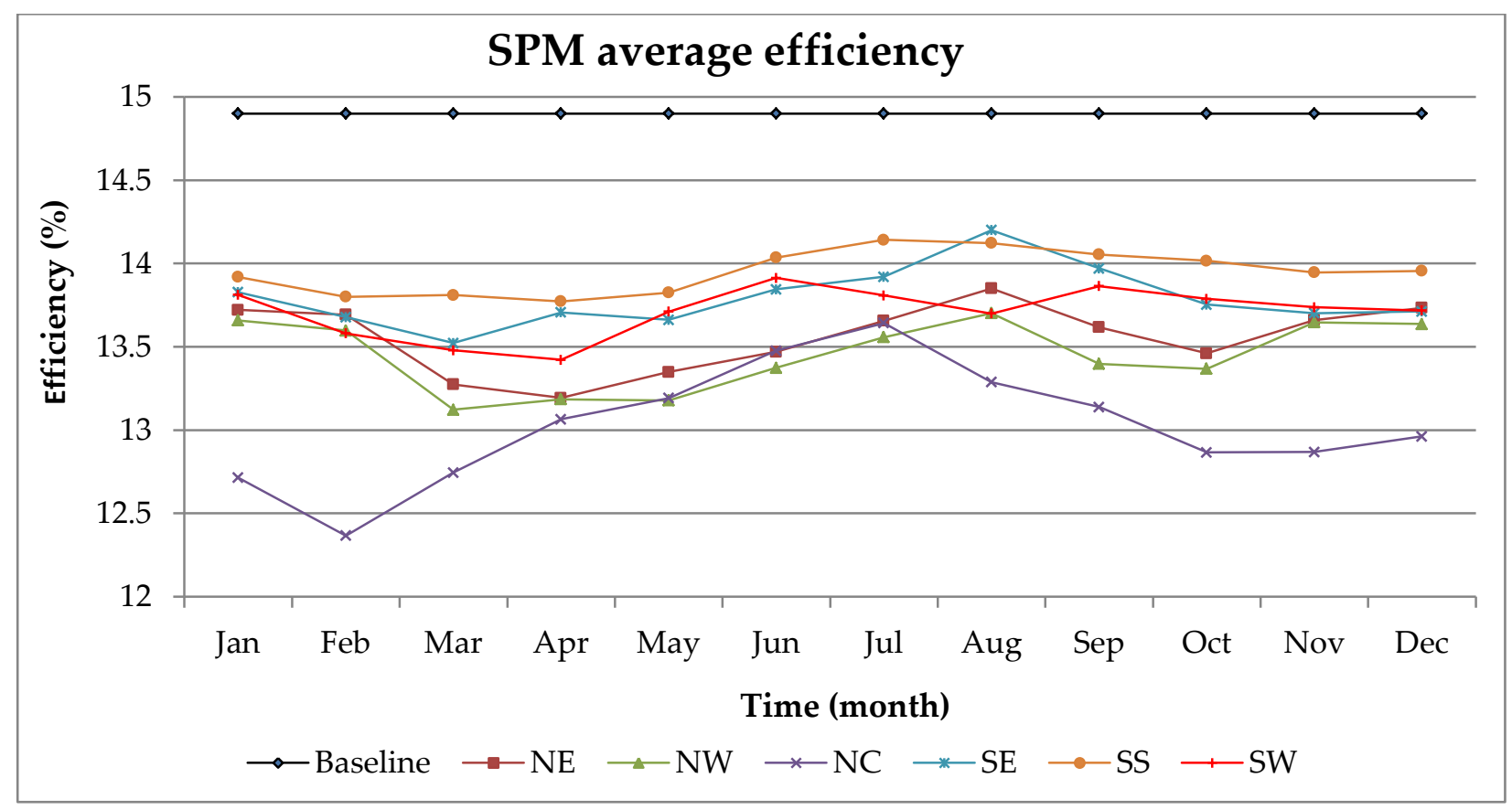

Figure 37: SPM systems' efficiency 


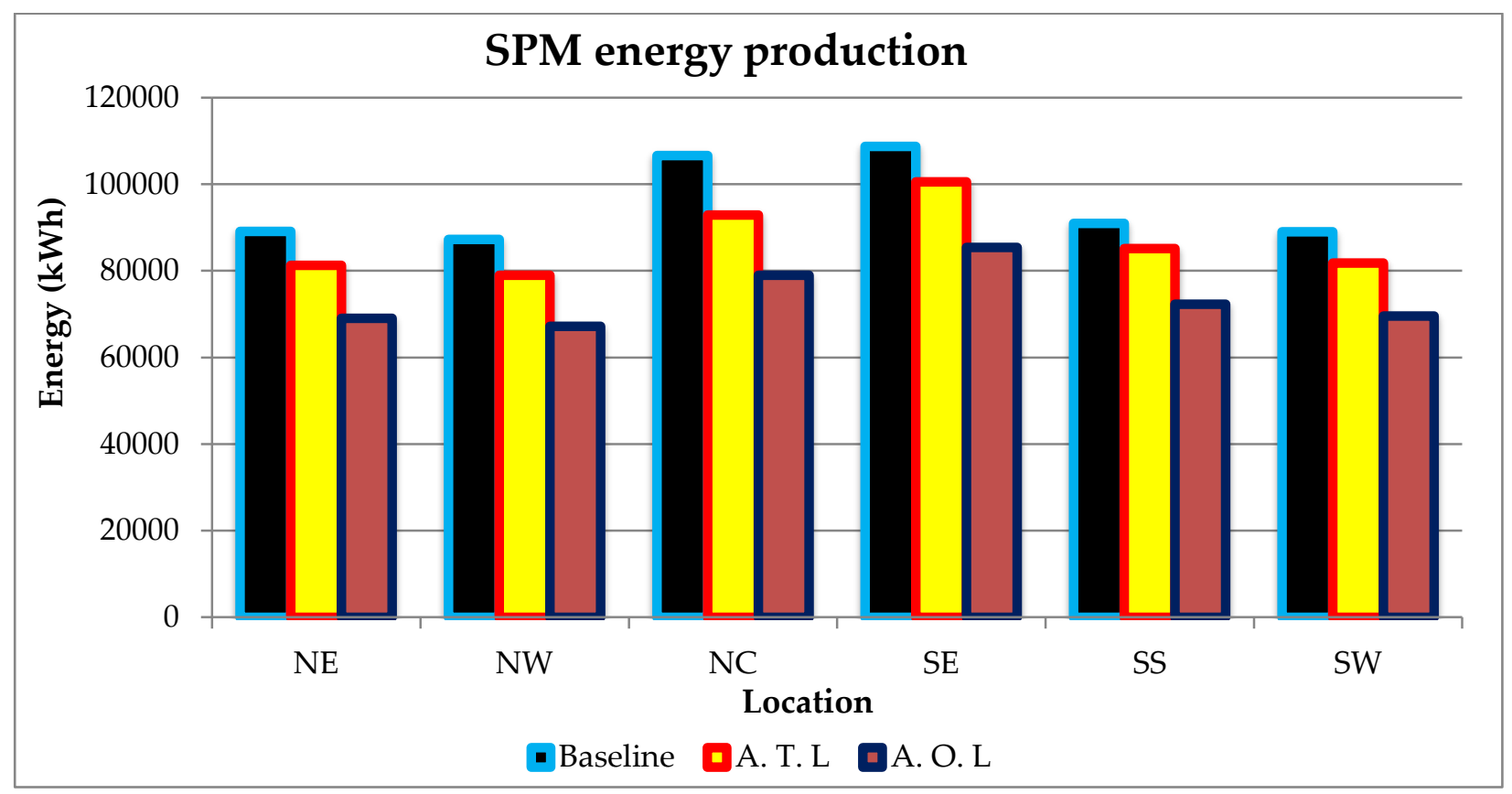

Figure 38: SPM systems' energy generation

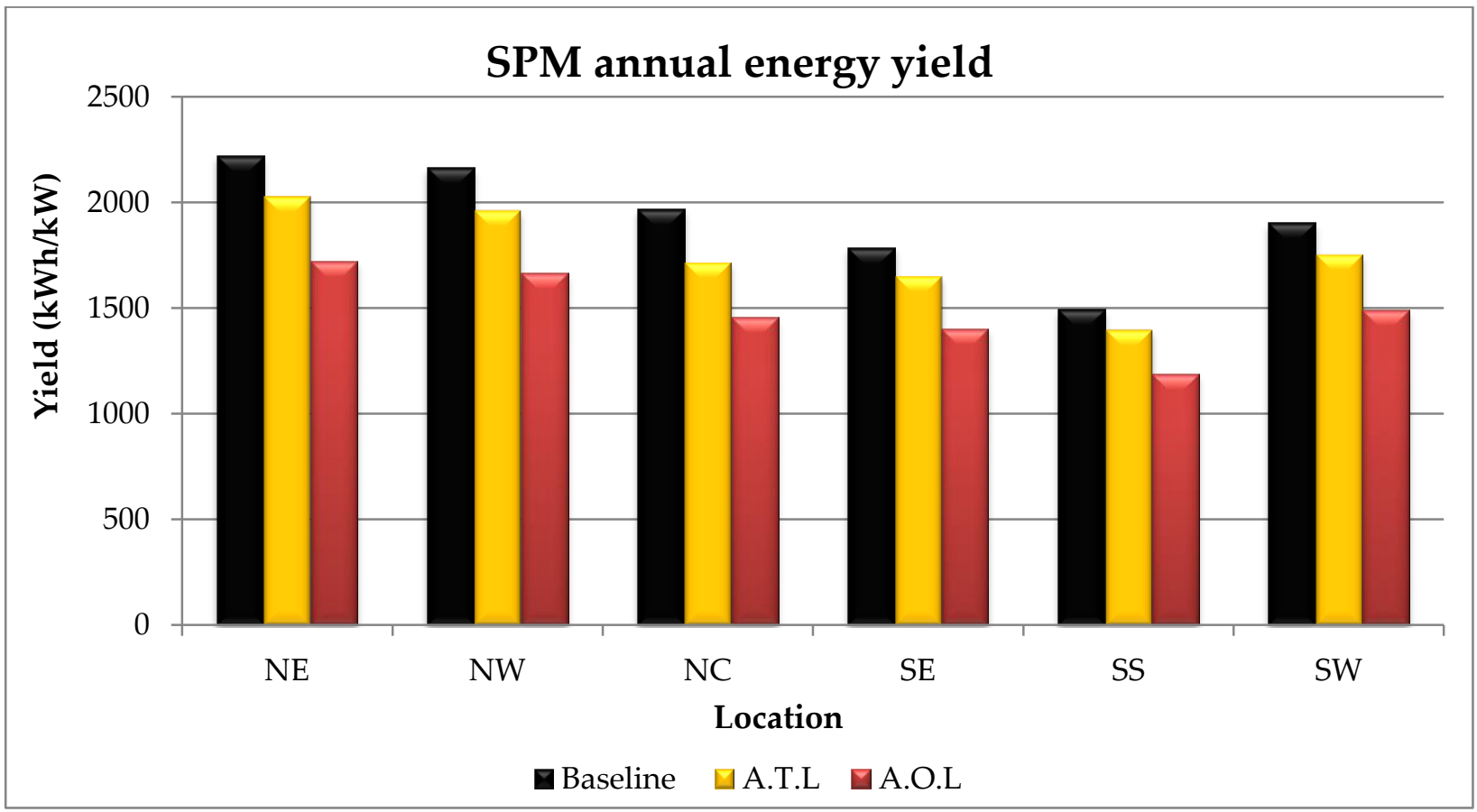

Figure 39: SPM systems' normalised yields 


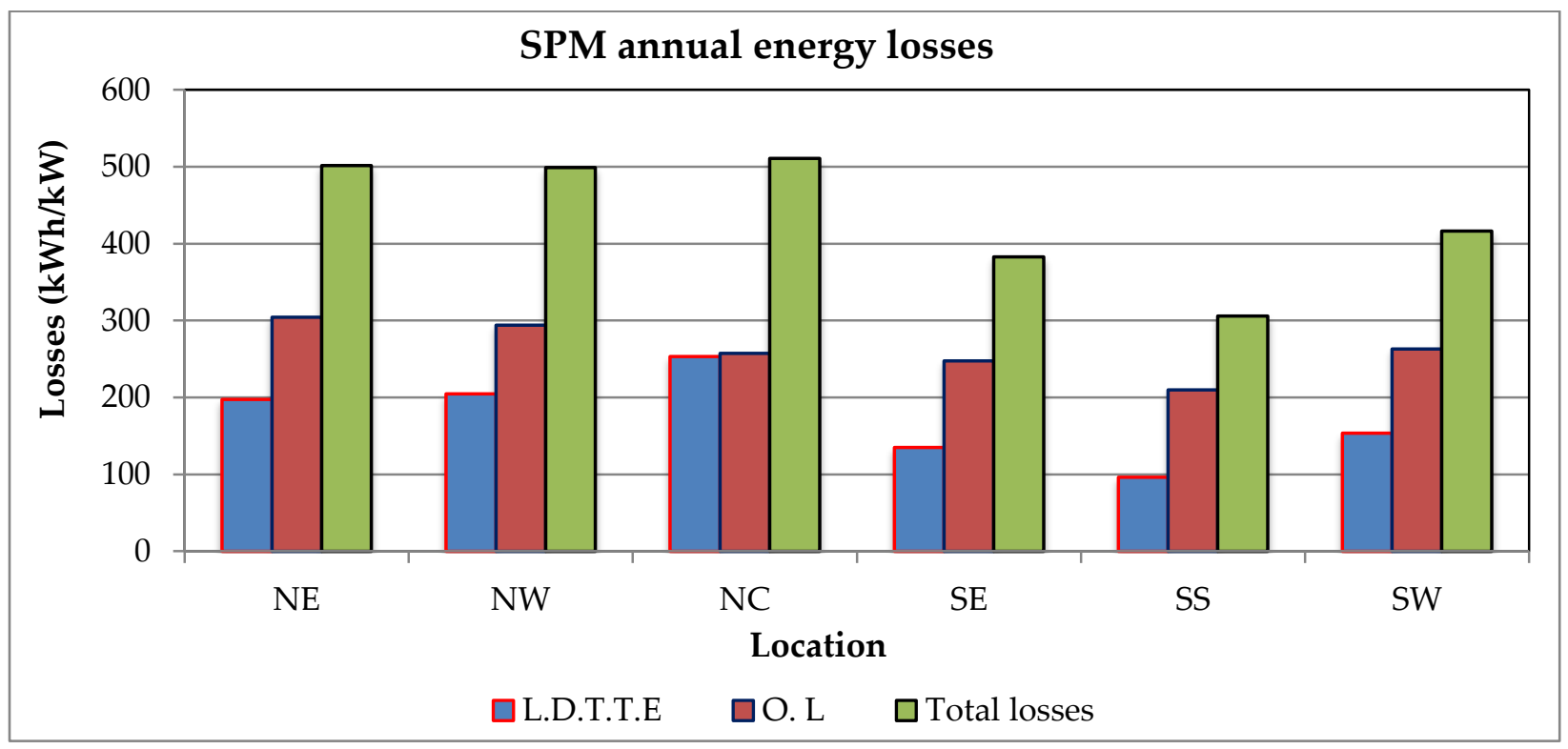

Figure 40: SPM systems' normalised losses

The analysis has revealed the amount of power, energy yields and losses that could be obtained from different photovoltaic microgrids in different communities of Nigeria's six geo-political zones. The results clearly demonstrate that the SPM systems in the southern zones of the country, i.e. SE, SS and SW locations, have relatively lower yearly energy losses compared to those in the northern zones, i.e. NE, NW and NC locations. A major reason for this disparity is that, though the northern region has a higher solar energy potential compared to the southern part, it experiences a relatively higher ambient temperature effect. This contributes to the higher temperature losses of the systems in the northern region than those in the southern part.

The values of the other losses for the northern zones are also higher than those of the southern zones. Furthermore, the yearly average efficiency of the systems in the SE, SS and SW zones are $13.79 \%, 13.95 \%$ and $13.71 \%$, while those in the NE, NW and NC are $13.56 \%, 13.45 \%$ and $13.03 \%$, respectively (see Table 13). The efficiencies of the systems in the southern zones are, therefore, higher than those in the northern zones.

These analyses, comparable to the results reported in [127, 178, 179, 180], validate the fact that lower ambient temperatures contribute to relatively lower energy losses and higher system's yields and efficiencies. It can, therefore, be inferred that the SPM's performance depends considerably on the location. The results presented in Table 13 
are comparable to the baseline and the final yields of $4.24 \mathrm{hr} / \mathrm{d}$ and $2.49 \mathrm{hr} / \mathrm{d}$, respectively, reported in [180] for a solar PV system in Hong Kong.

The final yields ranging from 0.41 to $3.28 \mathrm{hr} / \mathrm{d}$ have also been reported for a solar power generation system in Northern Ireland [181], with array losses of 0.79 - $0.84 \mathrm{hr} / \mathrm{d}$ and system losses of $0.23-0.31 \mathrm{hr} / \mathrm{d}$. In addition, other annual final yields are reported in the International Energy Agency Photovoltaic Power System (IEA PVPS) Task 2 database, for Germany, Netherlands, Japan, Israel, Italy and France. These values are 1.8 $\mathrm{hr} / \mathrm{d}, 1.8 \mathrm{hr} / \mathrm{d}, 2.0 \mathrm{hr} / \mathrm{d}, 2.7 \mathrm{hr} / \mathrm{d}, 3.5 \mathrm{hr} / \mathrm{d}$ and $1.75 \mathrm{hr} / \mathrm{d}$, respectively [181, 182]. Italy has a higher mean yield because of its relatively higher average irradiation compared to the other locations.

In addition, the SPM's yields and losses analysis results can also be compared with the final yields of 1.45 to $2.84 \mathrm{hr} / \mathrm{d}, 3.73 \mathrm{hr} / \mathrm{d}, 4.24 \mathrm{hr} / \mathrm{d}$ and $3.09 \mathrm{hr} / \mathrm{d}$ reported for photovoltaic power generation plants in Khatkar-Kalan, Karnataka State India, Nagpur and Assansol in India, respectively [183, 184]. Also, the literature reports the final yields of $3.7 \mathrm{hr} / \mathrm{d}$ and $1.96-5.07 \mathrm{hr} / \mathrm{d}$ for solar photovoltaic systems at the Unversity of Ma'laga Spain and the Island of Crete, Greece, respectively [127, 183]. The results presented in Table 9 can also be compared with the mentioned values.

These analyses and comparisons make it clear that achieving higher photovoltaic energy yields in different parts of Nigeria, is possible because of the country's relatively higher solar energy resources. The results, thus, provides useful knowledge of the design and performance of solar photovoltaic microgrid systems for different locations.

Furthermore, this thesis adds value to the body of knowledge by introducing detailed sizing methodologies and performance analyses (i.e. power output, energy production, yield and losses and the efficiency) for SPMs in different parts of Nigeria. These were not discussed by the studies documented in $[103,107,108,110,111,113,114$, 121]. Also, by carefully analysing the SPM's possible energy losses, the thesis performance evaluation results fill the identified knowledge gaps in those studies, including those reported in $[112,120,128]$.

This thesis, thus, critically demonstrates that the analysis of losses is a very important aspect of solar PV microgrid design and planning that should not be overlooked or underestimated. In addition, the inverter, mismatch and the wiring losses 
in Table 8 are categorised as BOS losses, while the losses due to dust or soiling, ageing etc., are part of the incomplete utilisation of solar irradiation [136, 143]. These two categories of losses are assumed to have almost equal value. Therefore, the value of each of the two losses may be obtained by dividing the $O$. Ls in Table 9 into two equal parts. With this assumption, it can be observed that the L. D. T. T. E has a higher proportion of the total losses compared to the O. Ls. This is typical of tropical regions, which is why the temperature effect is a significant factor that needs to be carefully considered for such regions, while the other losses are also being examined.

Table 13: Performance indices of the SPM systems

\begin{tabular}{|c|c|c|c|c|c|c|}
\hline Location & $\begin{array}{c}\text { Energy } \\
(\mathrm{kWh} / \mathrm{d})\end{array}$ & $\begin{array}{l}\text { Yield } \\
\text { (hr/d) }\end{array}$ & $\begin{array}{l}\text { L.D.T.T.E } \\
\text { (hr/d) }\end{array}$ & $\begin{array}{c}\text { O.L } \\
\text { (hr/d) }\end{array}$ & $\begin{array}{l}\text { Total } \\
\text { losses } \\
(\mathrm{hr} / \mathrm{d})\end{array}$ & $\begin{array}{c}\text { Average } \\
\text { Efficiency } \\
(\%)\end{array}$ \\
\hline $\mathrm{NE}$ & 189 & 4.72 & 0.54 & 0.83 & 1.37 & 13.56 \\
\hline NW & 184 & 4.57 & 0.56 & 0.81 & 1.37 & 13.45 \\
\hline $\mathrm{NC}$ & 216 & 3.99 & 0.69 & 0.70 & 1.40 & 13.03 \\
\hline SE & 234 & 3.84 & 0.37 & 0.68 & 1.05 & 13.79 \\
\hline SS & 198 & 3.26 & 0.27 & 0.57 & 0.84 & 13.95 \\
\hline SW & 190 & 4.08 & 0.42 & 0.72 & 1.14 & 13.71 \\
\hline
\end{tabular}

\subsection{Energy storage analysis}

The battery state of charge analysis for the different locations is presented in three different cases, such as the initial scenario, scenario 1 and scenario 2 .

- The initial scenario involves the analysis with the initial SPM capacity based on the location's lowest peak sun hours and the highest users' demand.

- The scenario 1 involves the analysis of the SPM's capacity if the initial capacity is unable to keep the battery SoC values above the set point of $30 \%$ for at least $\geq$ $95 \%$ of the time 
- The scenario 2 involves the analysis of the users' load reduction if the initial capacity is not able to keep the battery SoC values above the set point of $30 \%$ for at least $\geq 95 \%$ of the time

The $\geq 95 \%$ value is assumed in this study, as a good reference for the SoC distribution probability. The same battery system of $540.24 \mathrm{kWh}$ is used for all the zones. This battery configuration is based on a $50 \%$ DoD design (i.e. SoC of 50\%). The $50 \% \mathrm{DoD}$ is in agreement with the Trojan Battery design procedures for practical application of renewable energies for off-grid applications. The ultimate goal of such procedures is to ensure a long service life for the battery bank [156]. Many designers and planners in the field use this as a basis for developing stand-alone energy systems.

The battery is designed with a DoD of $50 \%$ and it also allows energy discharge up to $70 \%$. A difference of $20 \%$ is, therefore, being considered for contingencies, such as a sudden increase of users' load demands or decrease in the SPM's energy generation. A similar idea is demonstrated in [168], where the authors considered a reserve capacity of $10 \%$. The final battery set point will be an MDoD of $70 \%$ (i.e. minimum SoC of $30 \%$ ), beyond which the system will be considered to experience a loss of energy. The loss of energy is basically calculated in terms of the unmet load demand throughout the year. Different minimum battery set points have been reported in the literature; $20 \%$ in [159, $180,187,188], 30 \%$ in [189], 35\% in [168] and $40 \%$ in $[185,186]$.

A battery set point of $30 \%$ is used in this study for the technical analysis. This is also a standard set point introduced by the National Renewable Energy Laboratory (NREL), US in the Hybrid Optimisation Model for Electric Renewables (HOMER) microgrid simulation tool.

As stated in Chapter 3, the generation and the load analyses are based on the worst-case scenarios. This constraint, coupled with the derating and the temperature factors are introduced into the HOMER tool to examine the variations of the battery SoC with the solar irradiation cycles of the different locations. In situations where the results do not comply with the constraints, there are basically two options to address the challenges (see scenarios 1 and 2 above). Both options have their perceived shortcomings. While the increase of PV capacity is expected to increase the system's costs, the reduction of the load demands may also reduce the users' comfort or 
satisfaction [7]. However, the considerations are expected to guide in planning and decision-making. The three scenarios are examined and the battery analysis results are presented as follows:

1) Initial scenario

The average monthly battery state of charge for all the communities are shown in Figure 41. The system SoC values for the NE location are found to be around the set point of 30\% most of the time, especially between May and August. The battery system is operated on the margin, which indicates that the SPM is susceptible to a loss of energy, should there be a rise in demand or decrease in the energy supply. This is also the case with the system in the NW zone that has the SoC values for the majority of its months close to the $30 \%$ value (i.e. February to December).

The system SoC values of most of the months for the NC location are above the set point, with the lowest value of 35\% in July. The SE zone also has the majority of its SoC values above the set limit of $30 \%$, with the minimum SoC of $42 \%$ in August. Similarly, the system in SS zone has the lowest SoC value of $50 \%$ in July, while the system in the SW location has its lowest SoC value of 32\% in June, though some of its values are still above the set point.

The battery DMaps (data maps) of all the systems in the different zones are shown in Figures 42 to 47. These give the opportunity of observing the battery SoC's daily and seasonal patterns much more easily than with a simple time series plot. It can be seen in Figures 42 and 43 that the battery operates at SoC of 30-37\% (i.e. highlighted in black) mostly between about 12 midnight and 10 a.m. These values are minimal for the other hours of the day over the months. The SoC values are reduced to this level (i.e. 30 to $37 \%$ ) when the sun is unavailable, i.e. mostly in the evenings and nights. However, this is minimal during the day. There is the need to ensure the SoC values are not always found at this level for reliability purposes. The system profile for the NC zone is presented in Figure 44, which is better than those of the NE and NW locations. The SoC values between 30 and $37 \%$ are mostly obtained during the months with relatively lower solar irradiation cycle, i.e. between June and August.

The data map for SE is presented in Figure 45. This also has the SoC values of 30$37 \%$ during the months with relatively lower solar energy (i.e. August and a part of 
September). The profile for the SS zone is shown in Figure 46, which has lower values of $30-37 \%$, compared to the NE and NW zones. However, such values are relatively higher in the profile of SW shown in Figure 47. In the case of NE, NW and SW zones, all the months need to be considered to ensure the battery bank is operated above the values of $30-37 \%$ level most of the time. However, only those months with lower irradiance need to be considered for the systems in the SE, SS and NC zones.

The battery SoC's frequency probability is shown in Figures 48 (a) to (f). It is obvious that about $30 \%$ of the total SoC values are at $35 \%$ for the system in the NE zone (see Figure 48 (a)). Almost $45 \%$ of the SoC values are also at 35\% for the system in the NW zone, while about $9 \%$ of the total values are at $35 \%$ for the NC location (see Figures 48 (b) and (c)). Furthermore, only about 3\% of the total SoC occurrences are at 35\% for the SE community, while it is around 6.5\% for SS location (see Figure 48 (d) and (e)). Also, around $19 \%$ of the SoC values occur at $35 \%$ for the system in the SW zone (see Figure 48 (f)). The results demonstrate that all the average SoC values have very high probabilities of values $>30 \%$.

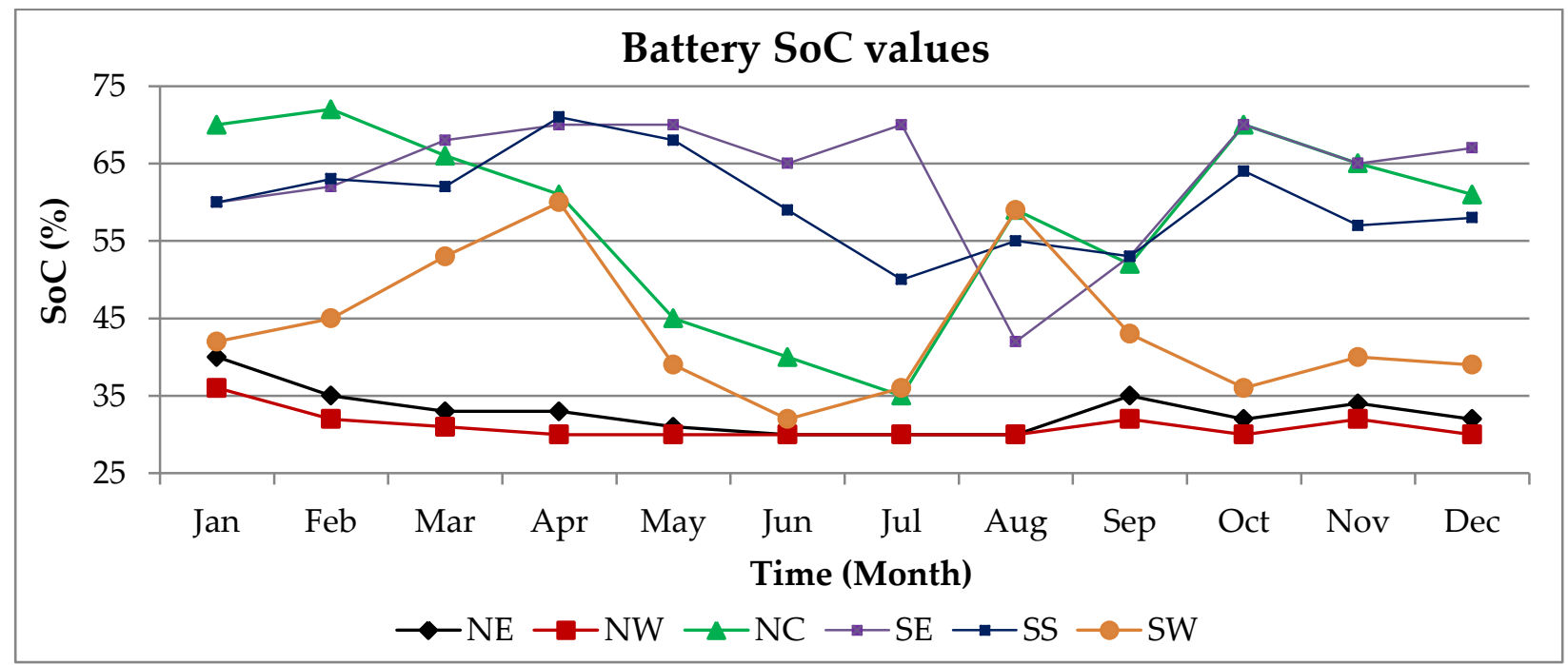

Figure 41: Battery SoC values for the initial scenario 


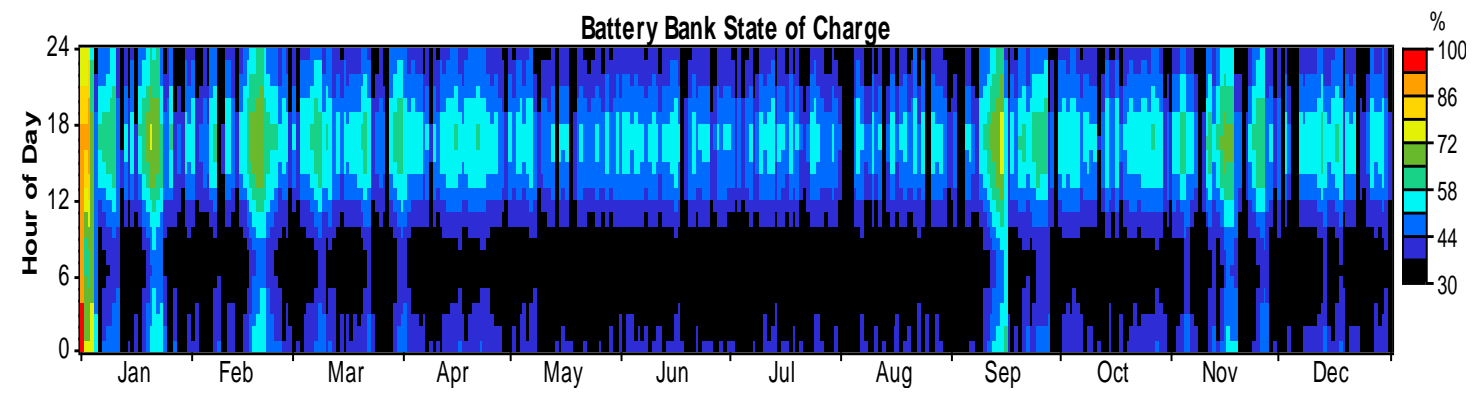

Figure 42: DMap of battery SoC for the NE zone

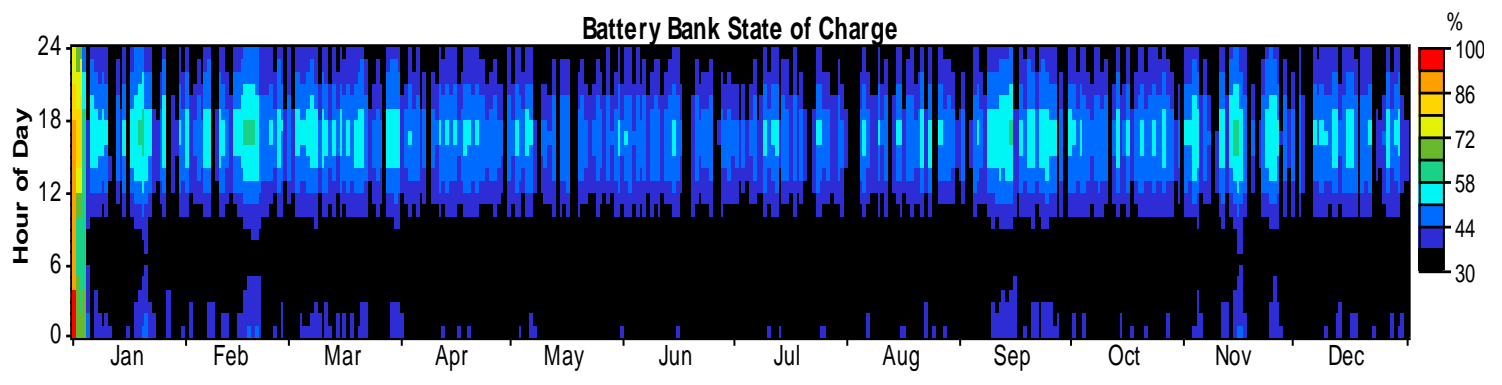

Figure 43: DMap of battery SoC for the NW zone

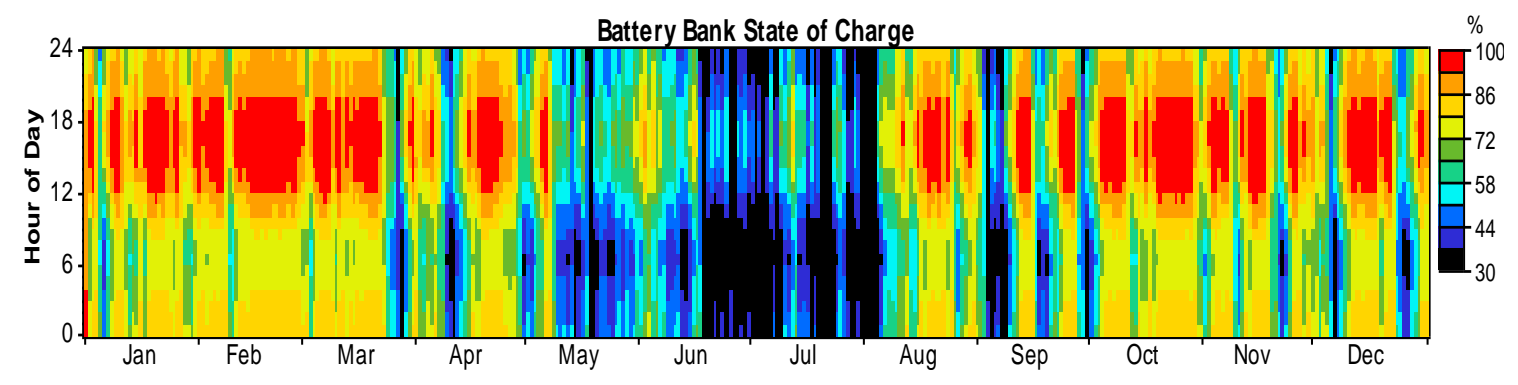

Figure 44: DMap of battery SoC for the NC zone

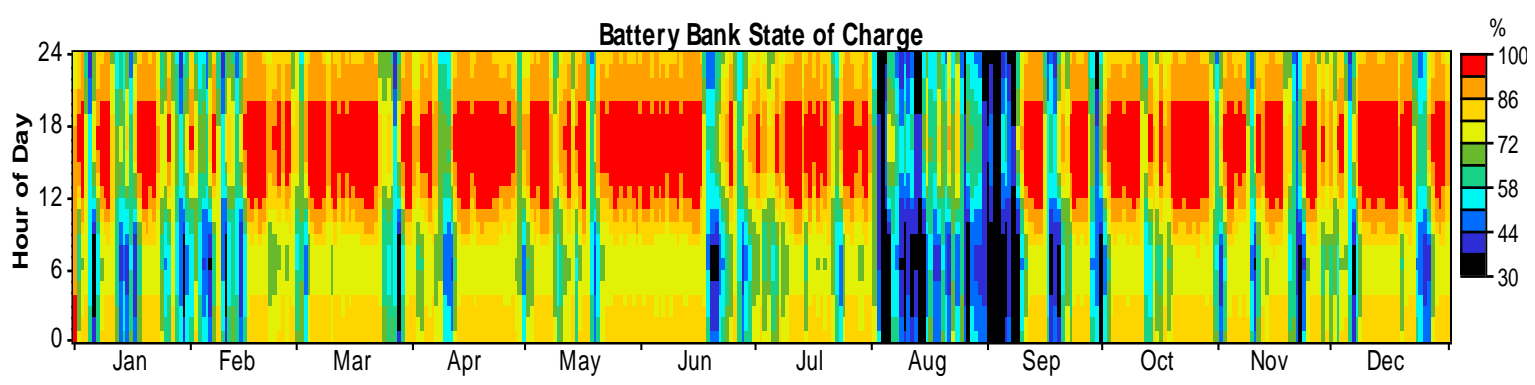

Figure 45: DMap of battery SoC for the SE zone 


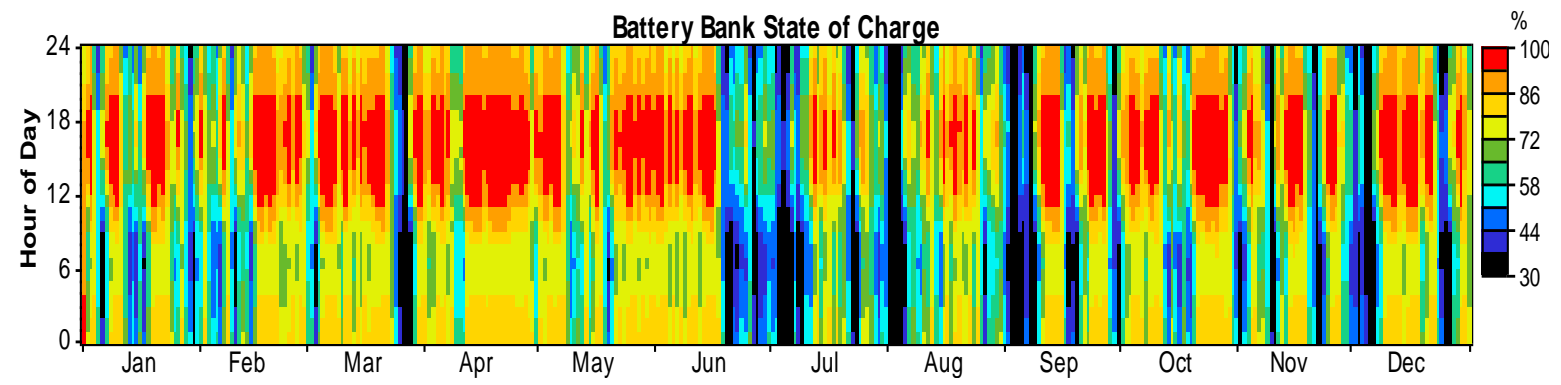

Figure 46: DMap of battery SoC for the SS zone

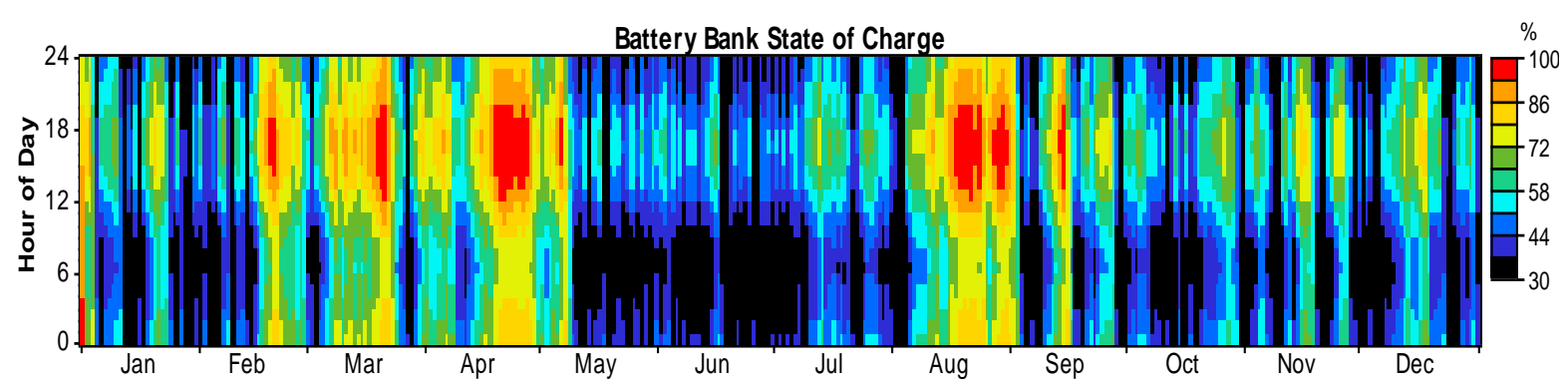

Figure 47: DMap of battery SoC for the SW zone

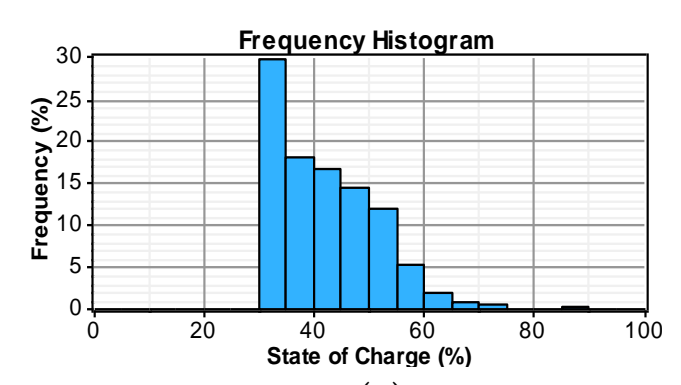

(a)

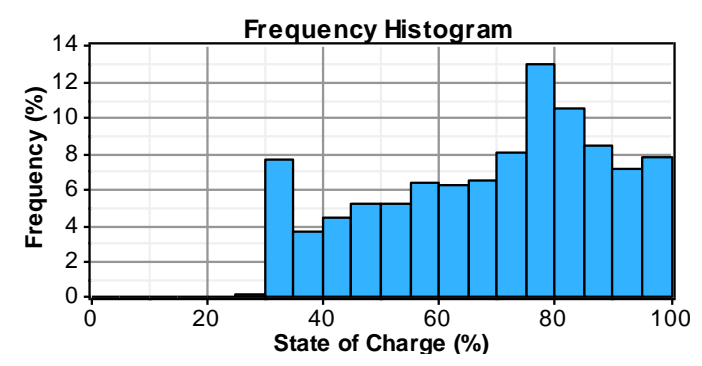

(c)

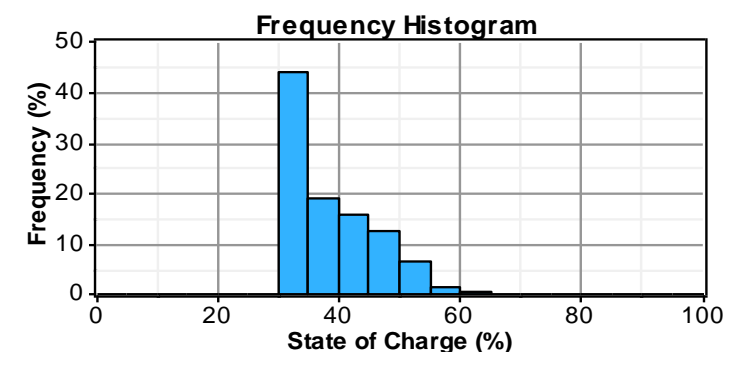

(b)

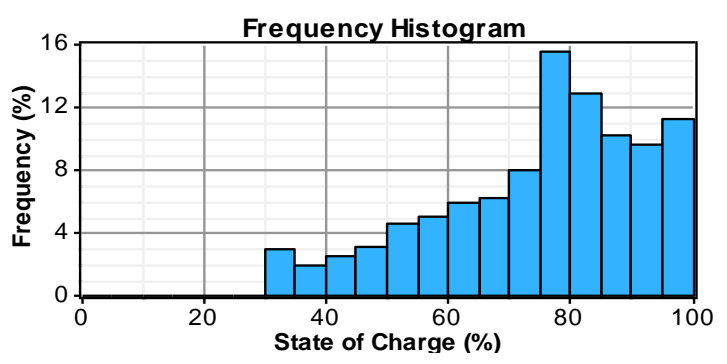

(d) 


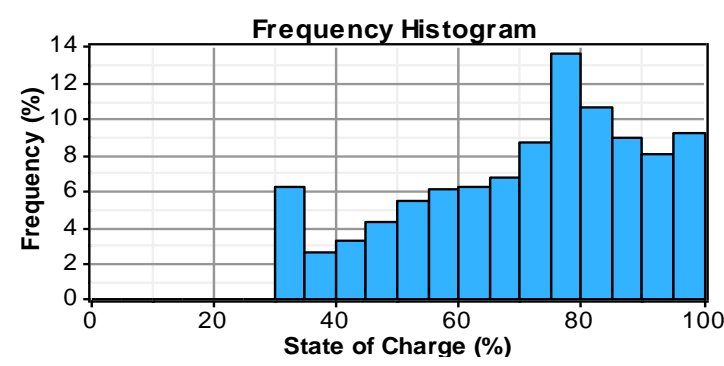

(e)

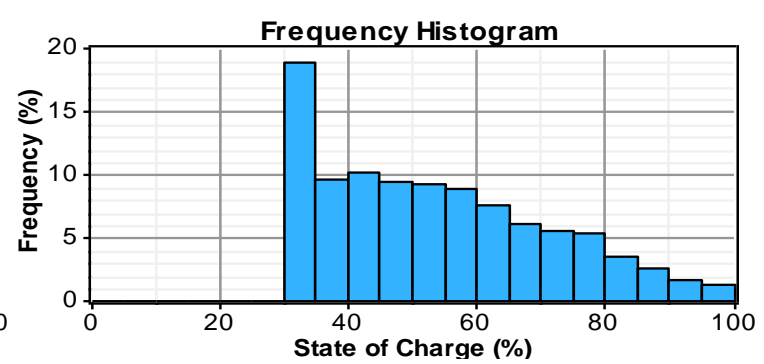

(f)

Figure 48 (a) - (f): Frequency distribution of the battery SoC

\section{2) Scenario 1}

The different SPM capacities for the scenario 1 are shown in Figure 49. The new system capacities for the NE, NW, NC, SE, SS and SW zones are 50, 55, 60, 65 and 63 and $56 \mathrm{~kW}$, respectively. The monthly battery SoC for the scenario 1 is also presented in Figure 50. It can be observed that the SoC values for the NE, NW, NC, SE, SS and SW zones have been increased compared to the initial values shown in Figure 41. The minimum SoC values for all the zones are 50\%, 43\%, 41\%, 48\%, 53\% and 53\%, respectively. These occurred in August, July, July, August, July and January, respectively.

The battery SoC data maps for the NE, NW, NC, SS and SW zones are presented in Figures 51 to 56. It is obvious that the SoC values of $30-37 \%$ (highlighted in black) have been minimised for all the zones. This is because the increase in the rated SPM's capacities allows more charging current to be supplied to the battery bank to achieve relatively higher SoC values. The DMap for the SS zone shown in Figure 55 is similar to the one presented in Figure 46 for the initial scenario. This is because most parts of the country's SS zone are riverine locations. Riverine areas in Nigeria are surrounded by water and they experience cloudy weather most times around the year. The DMap is unchanged because of this characteristic. For the same load conditions, the SS zone requires relatively higher PV capacity. This is why the energy profile of the battery does not show any difference like others.

The battery bank's frequency distributions for the zones are shown in Figures 57 (a) to (f). It can be seen that only $\sim 1 \%$ of the SoC values are at $35 \%$ for system in the NE zone, while they are $\sim 3 \%$ for the system in the NW zone. Also, the values are $\sim 4.5 \%$ for the system in the NC; $\sim 2.5 \%$ for the system in the SE zone, while they are $\sim 5 \%$ for both 
the SS and the SW zones. These results indicate that there is a higher probability of achieving the $\mathrm{SoC}>30 \%$.

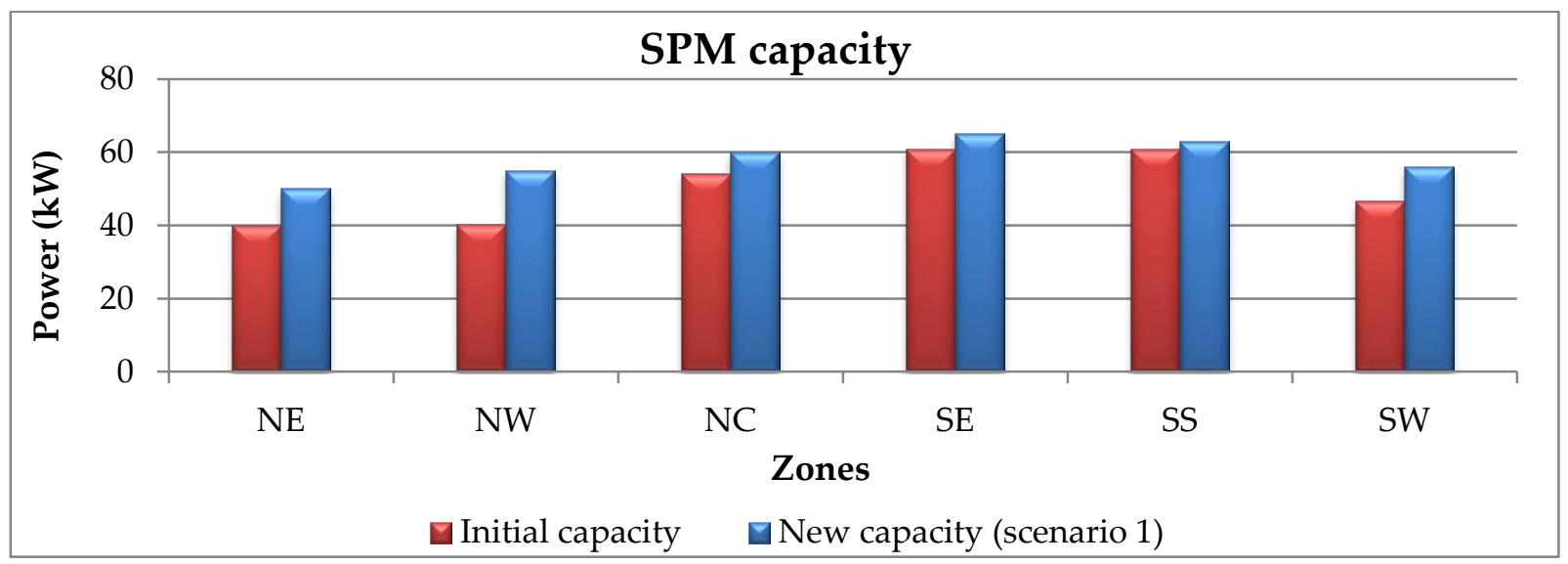

Figure 49: Rated SPM capacities

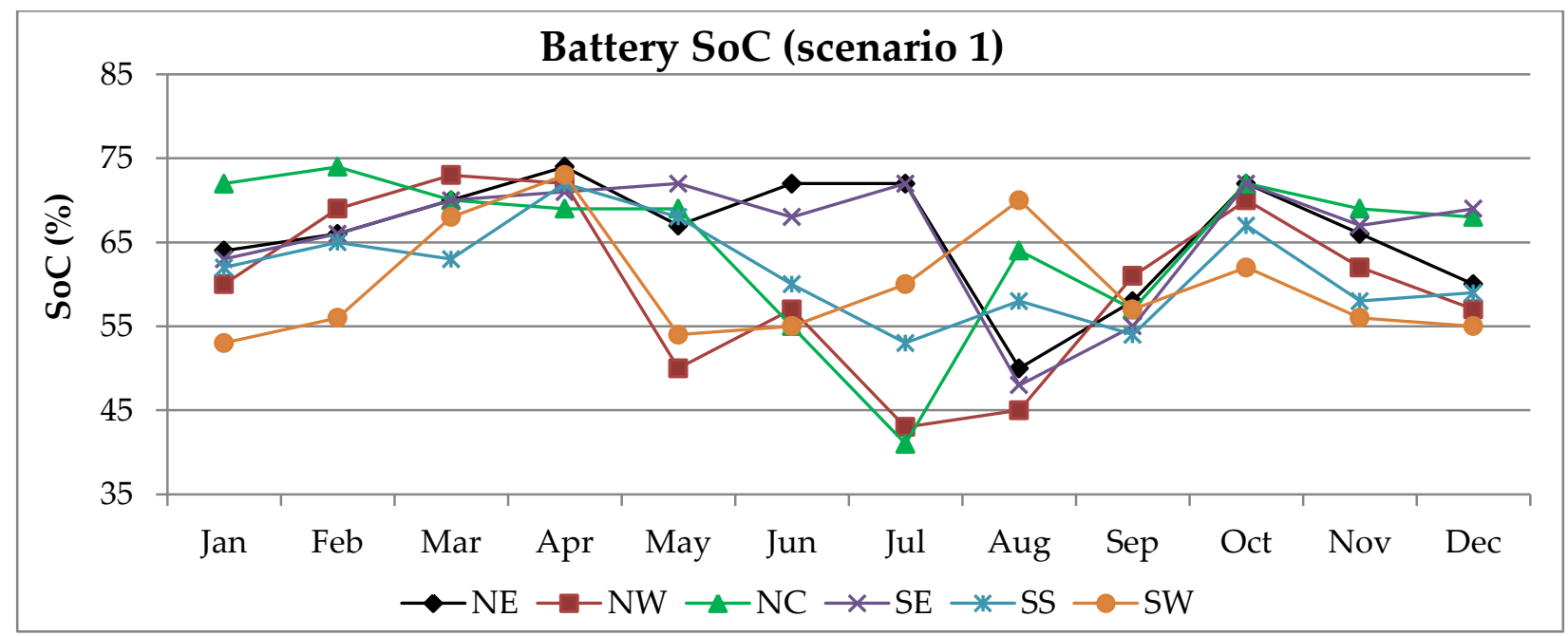

Figure 50: Battery SoC values for the scenario 1

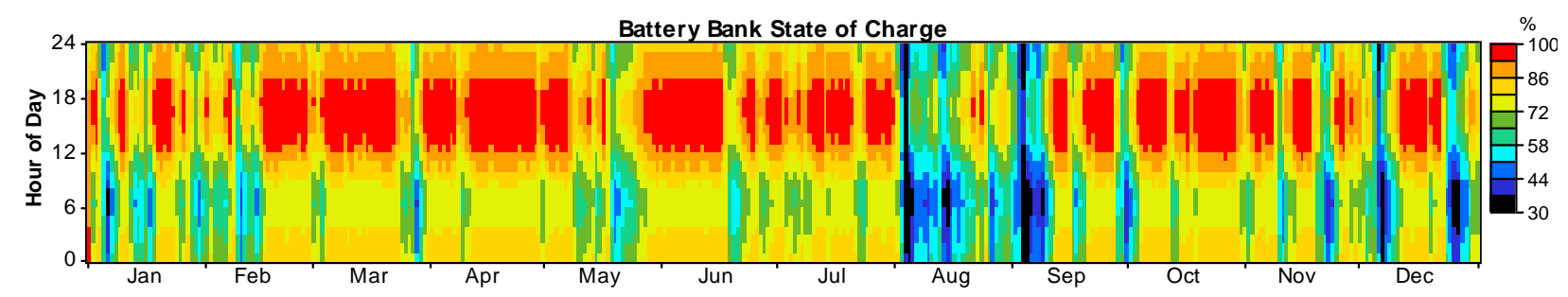

Figure 51: Scenario 1 battery SoC DMap for the NE zone 


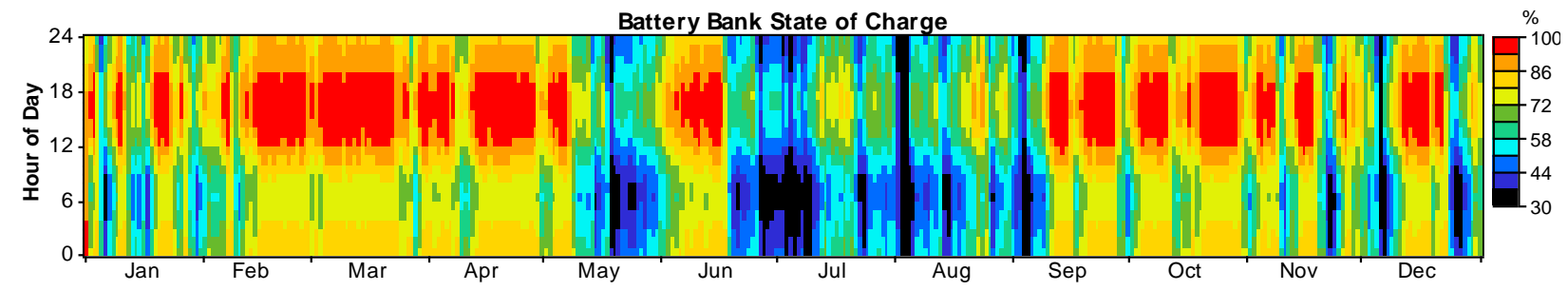

Figure 52: Scenario 1 battery SoC DMap for the NW zone

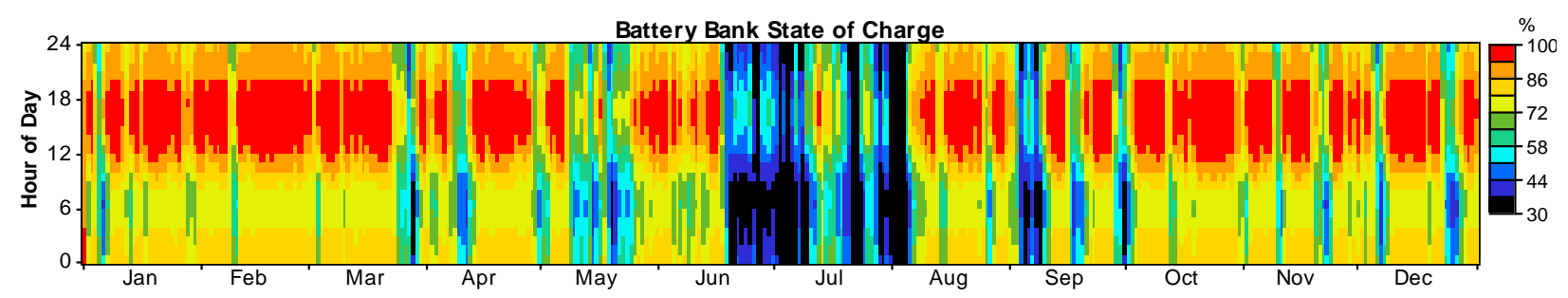

Figure 53: Scenario 1 battery SoC DMap for the NC zone

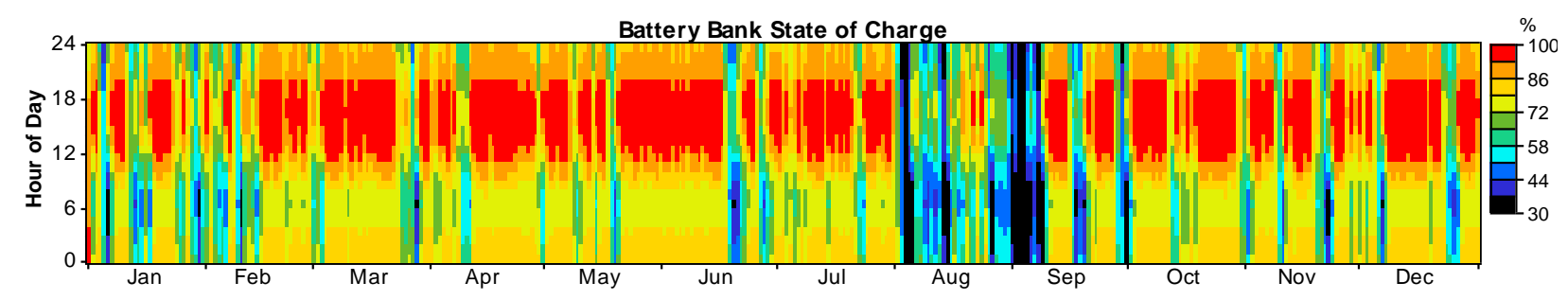

Figure 54: Scenario 1 battery SoC DMap for the SE zone

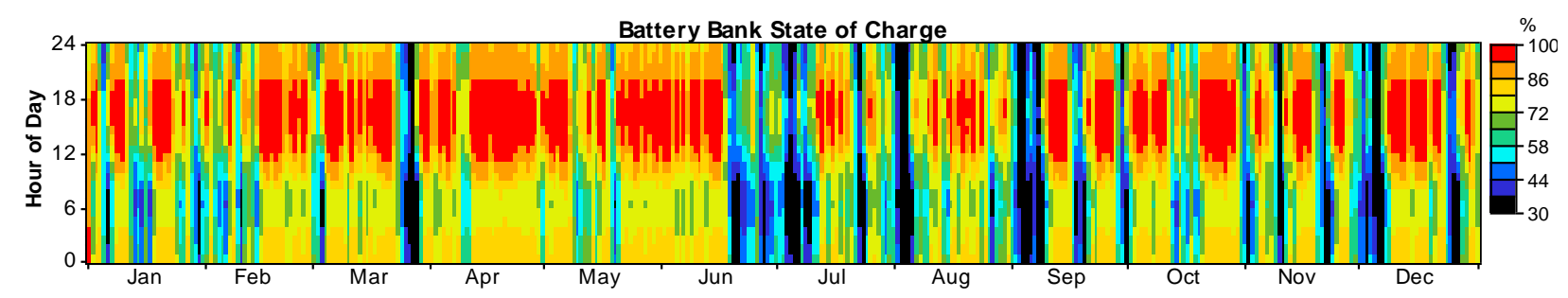

Figure 55: Scenario 1 battery SoC DMap for the SS zone

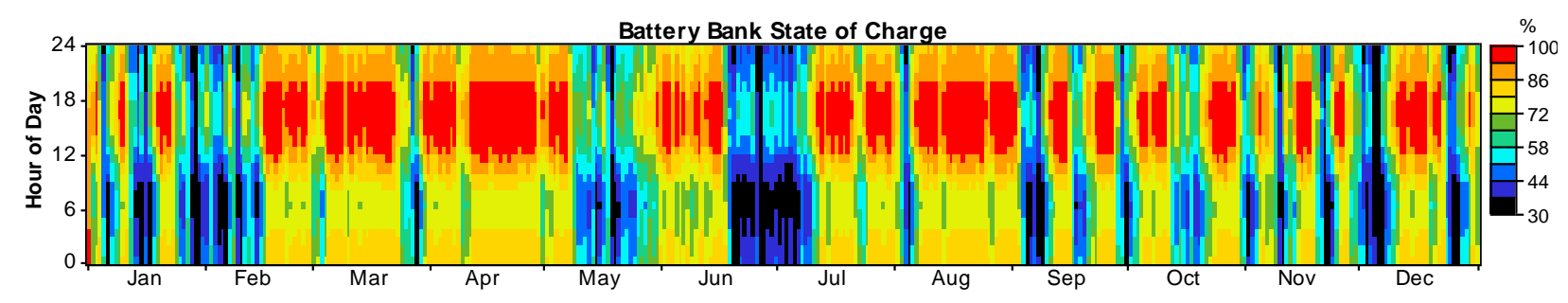

Figure 56: Scenario 1 battery SoC DMap for the SW zone 


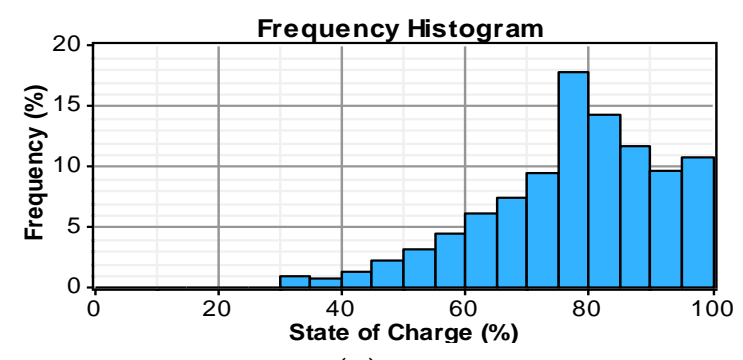

(a)

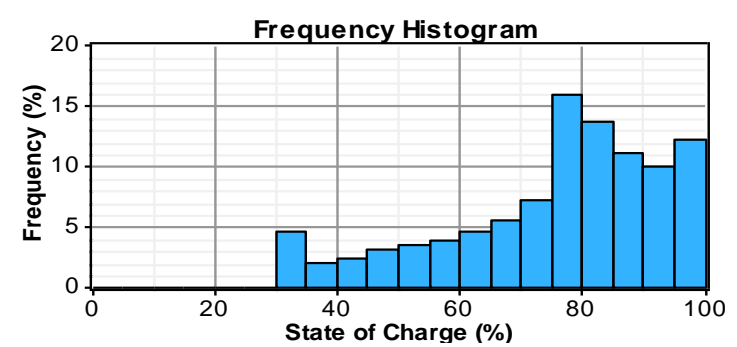

(c)

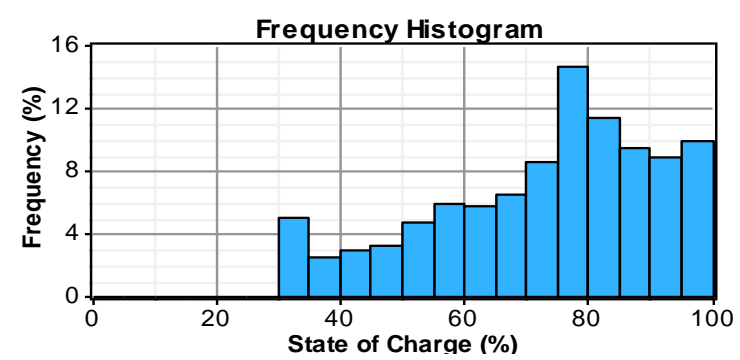

(e)

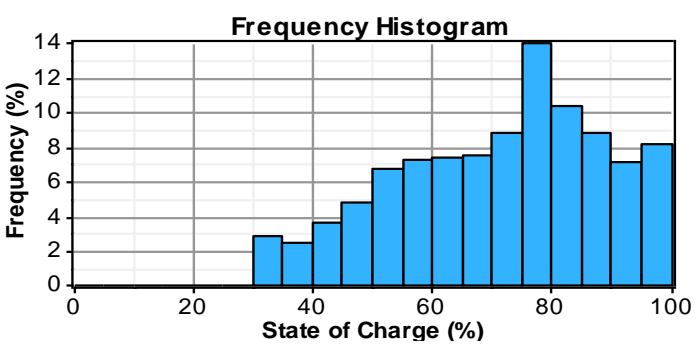

(b)

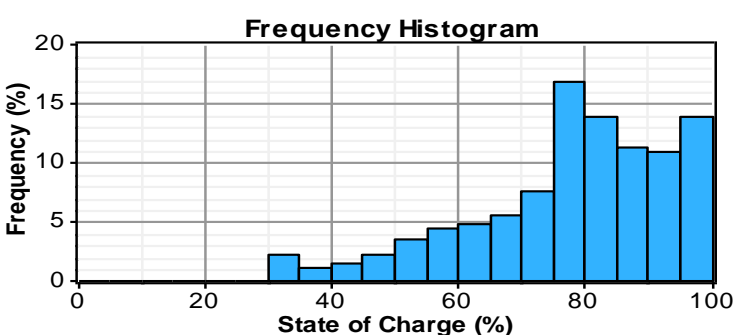

(d)

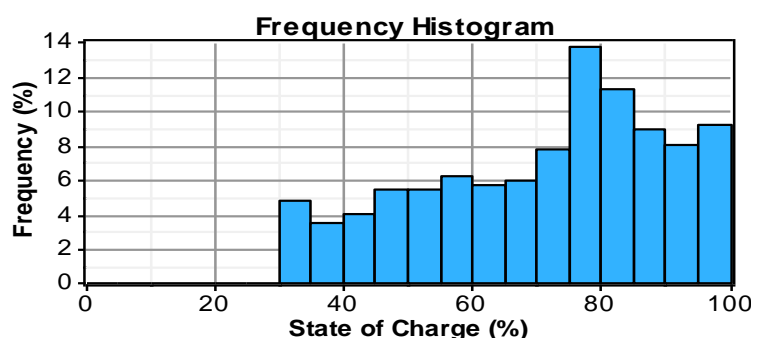

(f)

Figure 57 (a) - (f): Frequency distribution of SoC

\section{3) Scenario 2}

The battery SoC for $5-25 \%$ load demand reduction is shown in Figures 58 to 62, still using the initial SPM capacities. Though the SoC values obtained for the SE zone in the initial scenario are considered appropriate, reduction of the users' energy consumption is still applied in July that has the lowest SoC values. Load demand reductions are considered for all the months for the systems in the NE, NW and SW zones. However, the users' demands are only reduced between May and July for the system in the NC zone, and only in July for the SS zone.

Figure 58 shows that the values of $34 \%, 33 \%, 31 \%$ and $31 \%$ are obtained in May, June, July and August, respectively, for the system in the NE zone. These values are near to the set point. Also, the battery SoC of most of the months in the NW zone is close to the set point, except January that has a value of $38 \%$. It can also be observed that 
the system in the NC zone has higher SoC values in most of the months. The lowest SoC value of $47 \%$ is obtained in July for SS zone, while the lowest value of 33\% is obtained in June for the SW zone.

Furthermore, Figure 59 demonstrates that the system $\mathrm{SoC}$ values are still at the set point in April, May, June, July, August, October and December. The lowest SoC values for the system in the NE, NC and SW zones are 32\%, 40\% and 37\%, respectively. Similarly, in Figure 60, the SoC values of the other months have improved, but the battery is at the set point in May, June, July and August, for the NW zone. The SoC values between June and August are also very close to the set point for the NW zone (see Figure 61). However, the lowest values of 38\% and 39\% are obtained for July and August for the $25 \%$.

Some conclusions, can, therefore, be drawn from this analysis using fridges as the main appliance for the load reduction scenario. The fridges are the only appliances that are run as a base load in the design (i.e. they are operated for 24 hours in a day). This is because they present the highest energy requirements amongst the users' loads. The suitable time for the load demand reduction is during the night when the fridges will not be opened by the users as frequently as during the day.

- By applying a minimum load demand reduction of $15 \%$ to the system in the NE zone, the battery will be operated above the minimum SoC of $30 \%$ most of the time. The $15 \%$ demand reduction means that the initial users' demand of 174.98 $\mathrm{kWh}$ is reduced to $148.73 \mathrm{kWh}$. The difference is $26.25 \mathrm{kWh}$, which is equivalent to switching off the 24 fridges in the community for about 4.5 hours per day (the total load capacity of the 24 fridges is $6 \mathrm{~kW}$ )

- By applying a minimum load demand reduction of $25 \%$ to the system in the NW zone, the battery will be operated above the minimum SoC of $30 \%$ most of the time. The $174.98 \mathrm{kWh}$ daily load demand will be reduced to $131.24 \mathrm{kWh}$; the difference being $43.74 \mathrm{kWh}$ and this means the fridges will be switched off for about 7 hours

- By applying a minimum load demand reduction of $15 \%$ to the system in the NC zone, for May, June and July only, the battery will be operated above the minimum SoC of $30 \%$ most of the time. The $15 \%$ demand reduction means that the initial value of $174.98 \mathrm{kWh}$ is reduced to $148.73 \mathrm{kWh}$. The difference between 
these two values $(26.25 \mathrm{kWh})$ is equivalent to switching off the 24 fridges in the community for about 4.4 hours per day

- By applying a minimum load demand reduction of $10 \%$ to the system in the SE zone, for August and September, the battery will be operated above the minimum SoC of $30 \%$ most of the time. This means that the initial value of 174.98 $\mathrm{kWh}$ is reduced to $157.48 \mathrm{kWh}$. The difference between these two values $(\sim 17.50$ $\mathrm{kWh}$ ) is equivalent to switching off the 24 fridges in the community for about 3 hours per day

- By applying a minimum load demand reduction of $10 \%$ to the system in the SS zone for July, the battery will be operated above the minimum SoC of $30 \%$ most of the time. This means that the initial value of $174.98 \mathrm{kWh}$ is reduced to 157.48 $\mathrm{kWh}$. The difference between these two values $(\sim 17.50 \mathrm{kWh})$ is equivalent to switching off the 24 fridges in the community for about 3 hours per day

- By applying a minimum load demand reduction of $15 \%$ to the system in the SW zone for the months, the battery will be operated above the minimum SoC of $30 \%$ most of the time. The $15 \%$ demand reduction means that the initial value of $174.98 \mathrm{kWh}$ is reduced to $148.73 \mathrm{kWh}$. The difference between these two values $(26.25 \mathrm{kWh})$ is equivalent to switching off the 24 fridges in the community for about 4.5 hours per day

The DMaps for the SoC values are shown in Figures 63 to 68. It can be seen that the SoC values of $30-37 \%$ are minimal, compared to the initial scenario presented in Figures 42, 43, 44, 46 and 47. Also, the battery SoC frequency distributions are presented in Figures 69 (a) to (f). It can be seen that only $\sim 2 \%, \sim 4 \%$, $4 \%, \sim 1.5 \%, \sim 5 \%$ and $\sim 4 \%$ of the SoC values occur at the battery SoC of $35 \%$. This indicates that the obtained average battery SoC values are greater than the battery set point of $30 \%$ all the time. 


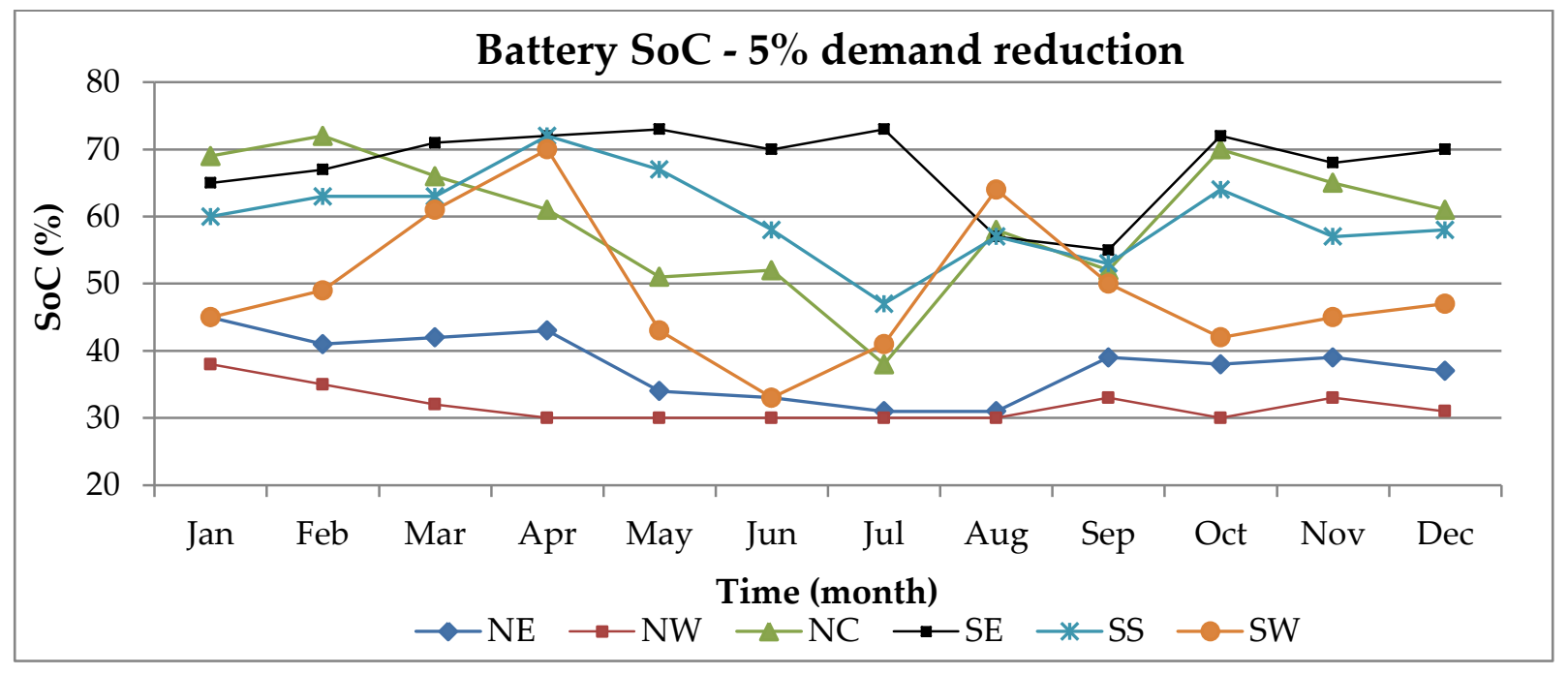

Figure 58: Battery SoC for 5\% load demand reduction

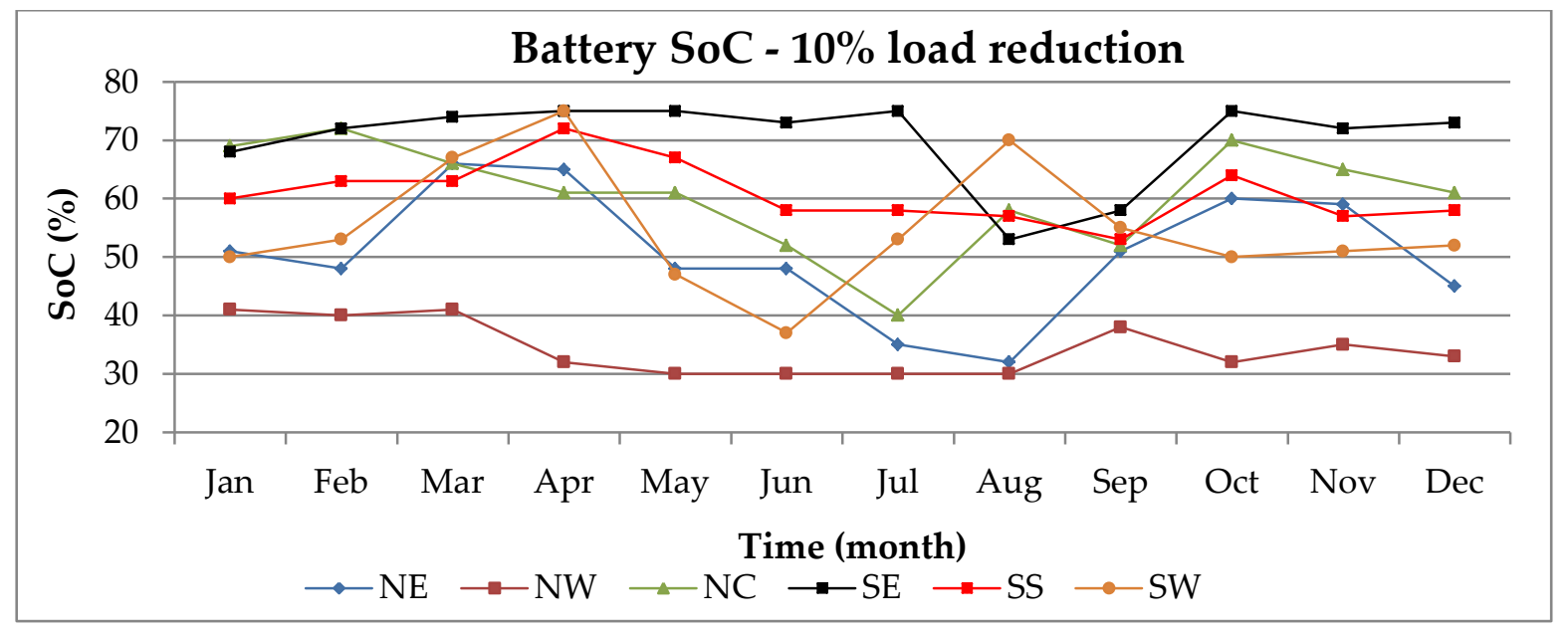

Figure 59: Battery SoC for 10\% load demand reduction 


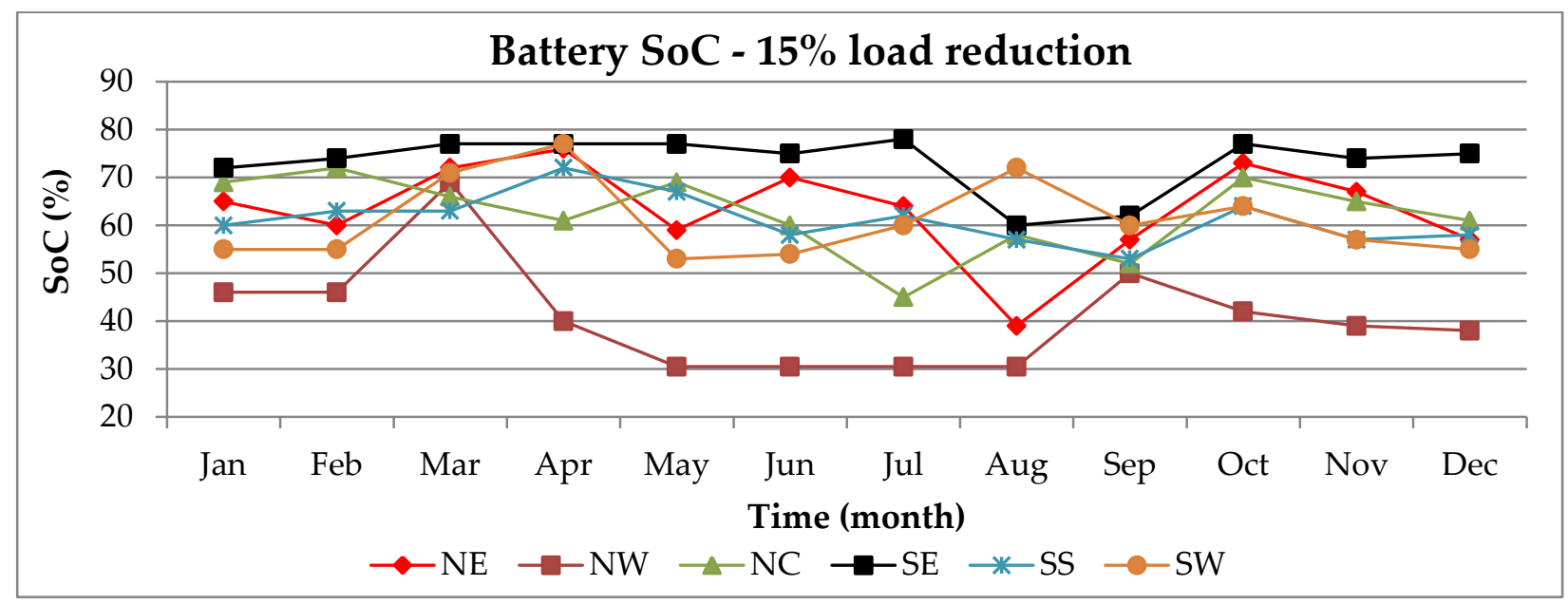

Figure 60: Battery SoC for $15 \%$ load demand reduction

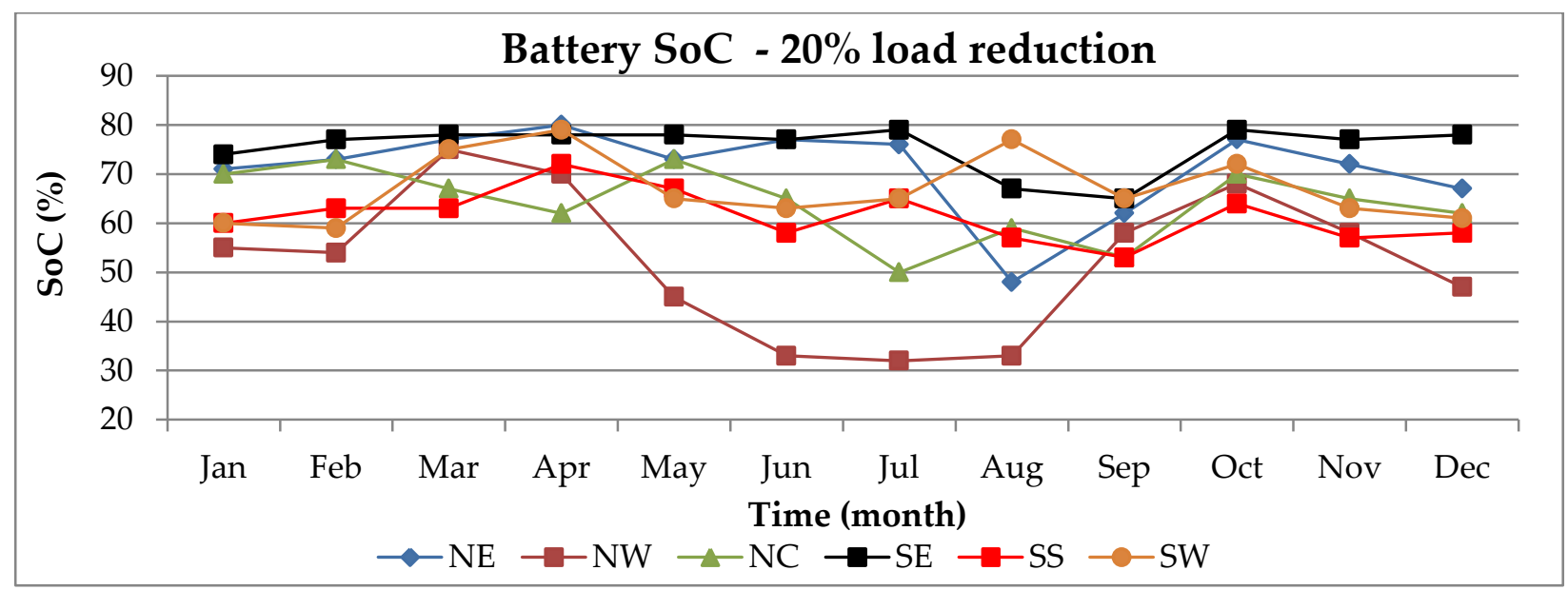

Figure 61: Battery SoC for 20\% load demand reduction

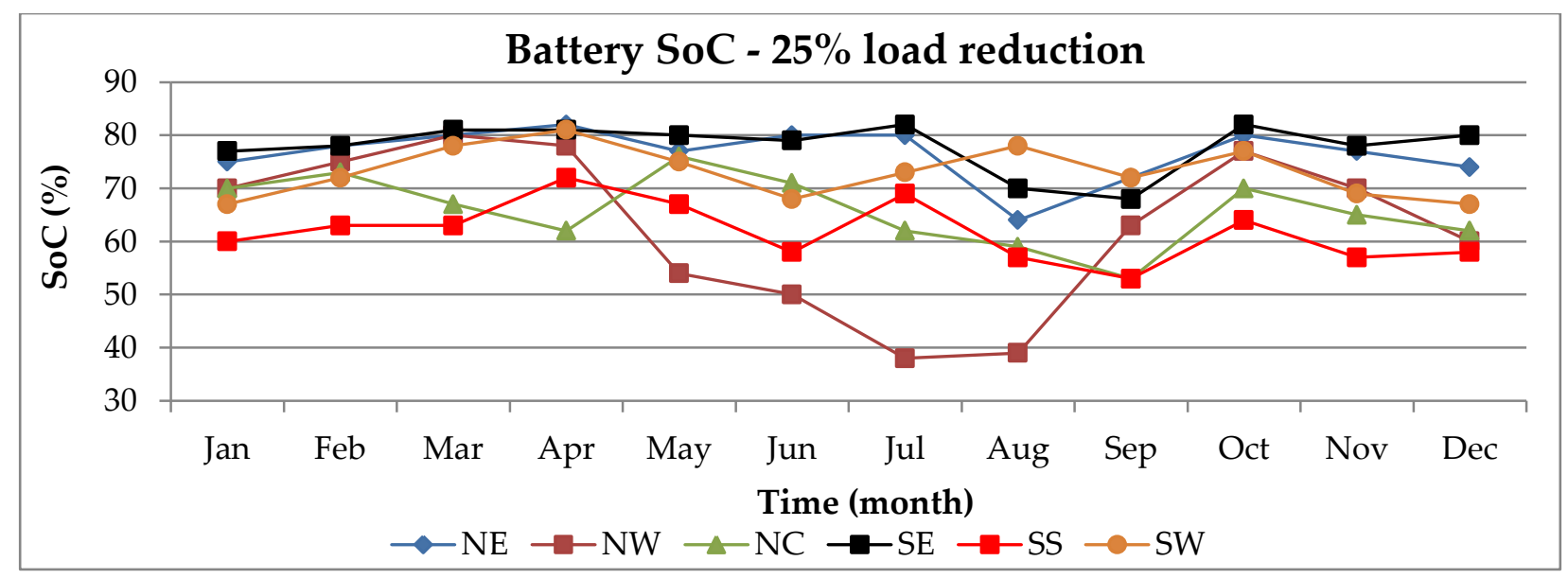

Figure 62: Battery SoC for 25\% load demand reduction 


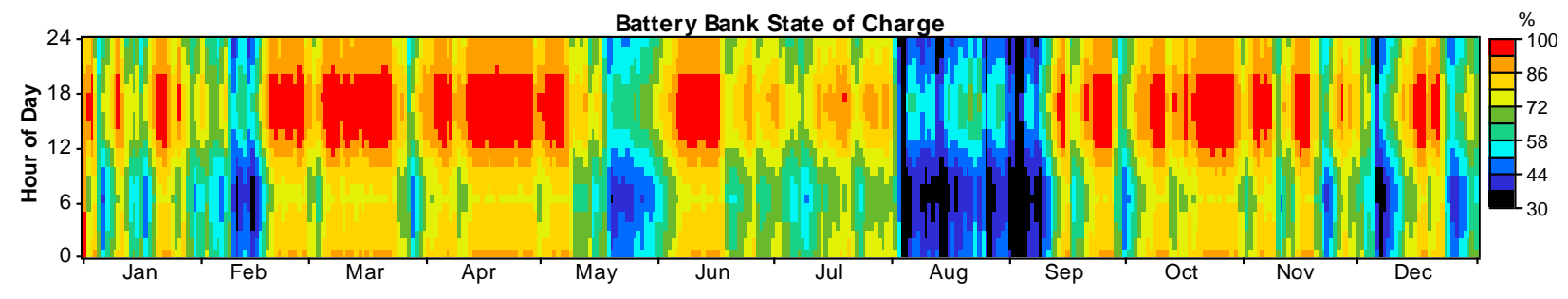

Figure 63: Scenario 2 battery SoC DMap for NE zone

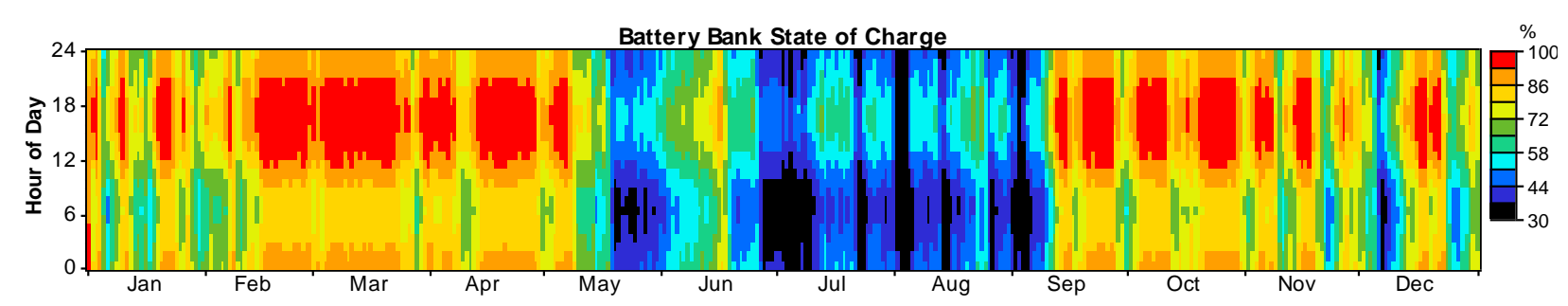

Figure 64: Scenario 2 battery SoC for NW zone

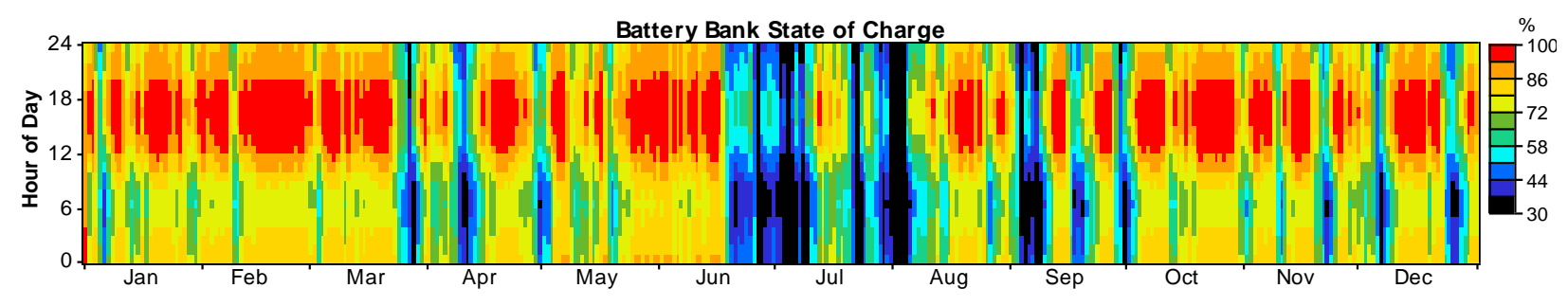

Figure 65: Scenario 2 battery SoC DMap for NC zone

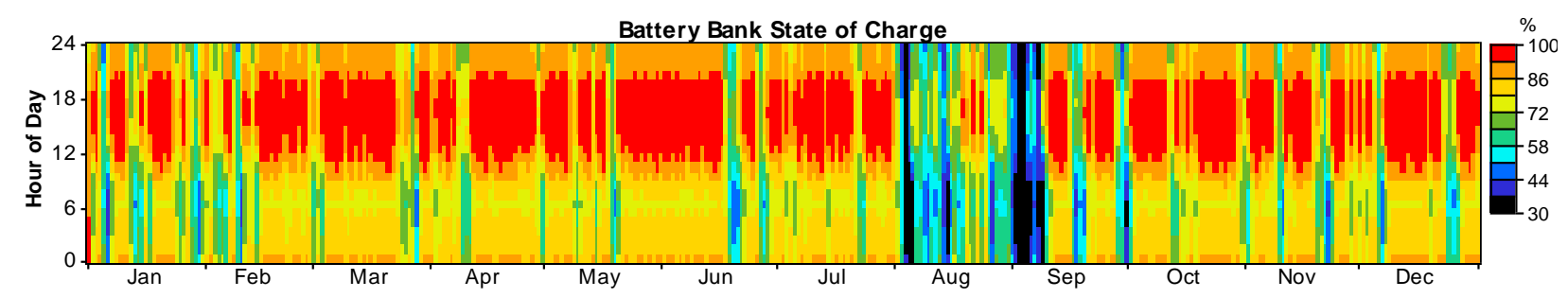

Figure 66: Scenario 2 battery SoC DMap for SE zone

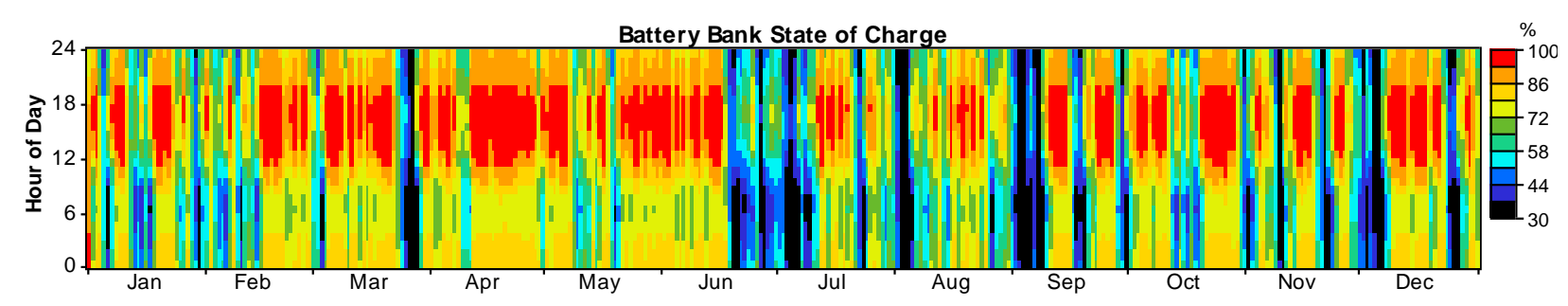

Figure 67: Scenario 2 battery SoC DMap for SS zone 


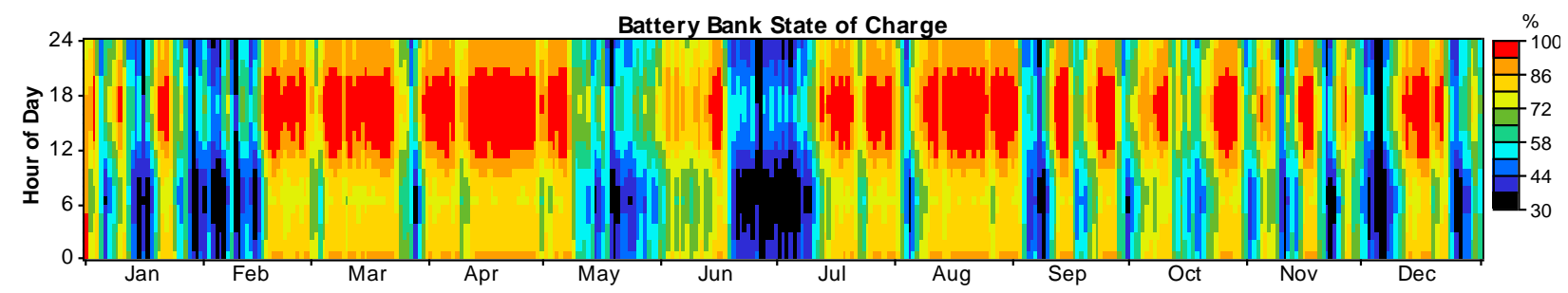

Figure 68: Scenario 2 battery SoC DMap for SW zone

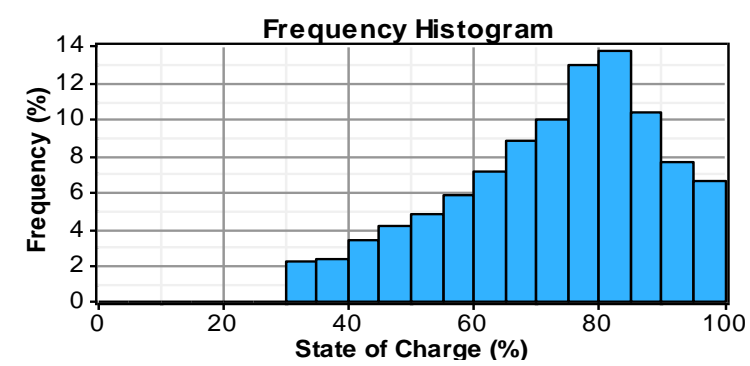

(a)

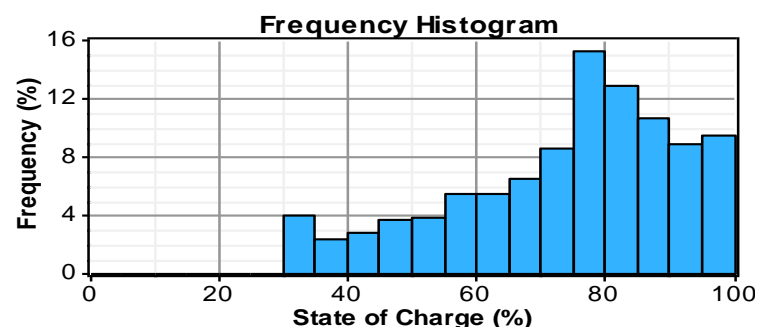

(c)

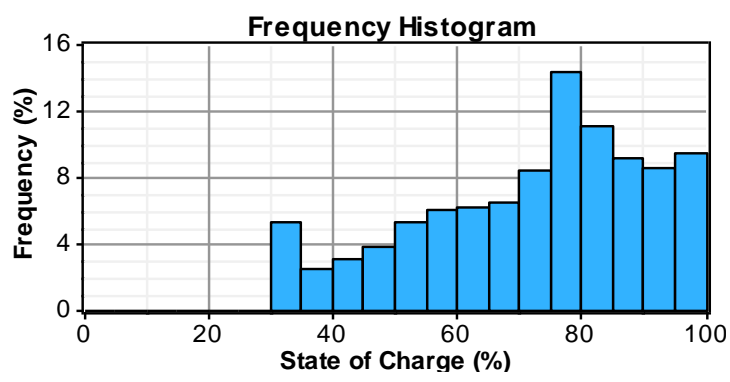

(e)

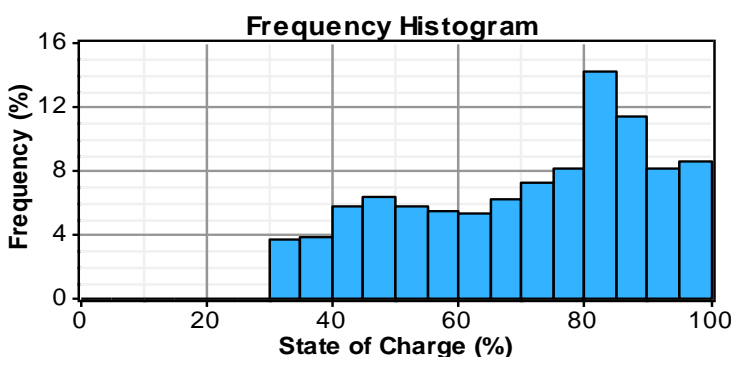

(b)

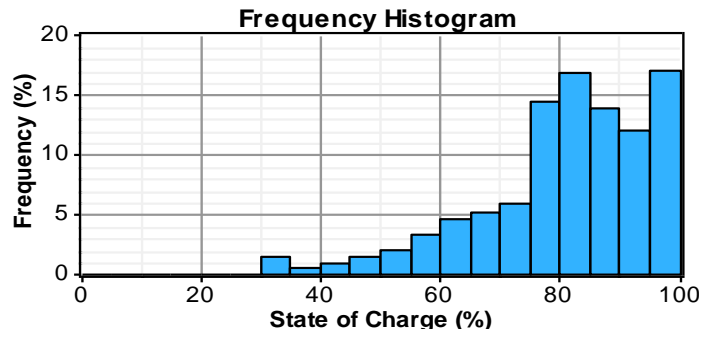

(d)

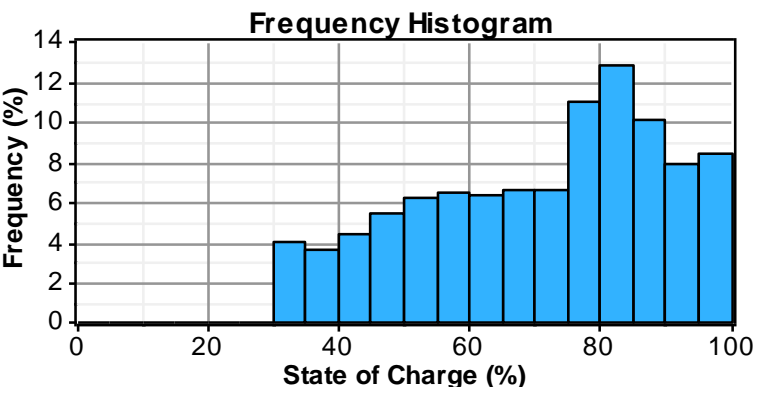

(f)

Figure 69: Frequency distribution of the SoC

The final SPM's energy production for the scenario 1 (i.e. $A O L$ value) is 86,138 $\mathrm{kWh}, 91,652 \mathrm{kWh}, 87,482 \mathrm{kWh}, 91,220 \mathrm{kWh}, 74,853 \mathrm{kWh}$ and 83,451 kWh for the NE, NW, NC, SE, SS and SW, respectively. These values are $19.86 \%, 26.73 \%, 9.75 \%, 4.2 \%$, $3.4 \%$ and $16.68 \%$ more than the energy production values obtained for the initial 
scenario. The losses obtained in the scenario 1 also differ from the initial scenario values by the same proportions mentioned above.

However, while there is a change in the values of the energy generated and the losses, in $\mathrm{kWh}$, the normalised yields and the losses for the initial scenario and the scenario 1 are the same (see Figures 39 and 40). These values are 1,723 kWh/kW, 1,666 $\mathrm{kWh} / \mathrm{kW}, 1,458 \mathrm{kWh} / \mathrm{kW}, 1,403 \mathrm{kWh} / \mathrm{kW}, 1,188 \mathrm{kWh} / \mathrm{kW}$ and 1,490 $\mathrm{kWh} / \mathrm{kW}$ for the systems in the NE, NW, NC, SE, SS and SW, respectively. In addition, they are also expressed as $4.72 \mathrm{hr} / \mathrm{d}, 4.57 \mathrm{hr} / \mathrm{d}, 3.99 \mathrm{hr} / \mathrm{d}, 3.84 \mathrm{hr} / \mathrm{d}, 3.26 \mathrm{hr} / \mathrm{d}$ and $4.08 \mathrm{hr} / \mathrm{d}$, which are the same with the results obtained for the initial scenario presented in Table 13.

\subsection{Hourly variations and reliability assessment}

The battery state of charge is also examined on the hourly basis to further test the SPM's effectiveness and the analysis. This is to guarantee a reliable energy supply for the intended users over the year. The hourly battery SoC values for all the zones are shown in Figures 70 to 75 . These results provide a better evaluation of the battery energy profile than the average values earlier presented. This is because it considers the energy supply in terms of the locations' intermittent solar energy resources.

The results demonstrate that the battery SoC values for scenarios 1 and 2 are better than those obtained for the initial scenario. A more conspicuous difference between the initial and the scenarios 1 and 2 can be seen in the graphs for the SPMs in the NE, NW and SW zones. However, such a difference is noticeable in the graphs of the systems in the NC, SE and SS zones for the periods with relatively lower irradiation. Furthermore, those parts of the figures where there are lower hourly $\mathrm{SoC}$ values represent the periods of the year when there is lower solar irradiation.

The purpose of the Solar Photovoltaic Microgrid (SPM) model is to meet the users' energy requirements. As part of the techno-economic design, it is necessary to test it whether or not it will be able to support the users' energy demand throughout the year [194]. This is achieved in this thesis through the unmet load demand, loss of energy probability and the availability indices, according to the IEEE 1562 standard [131]. The test allows the energy designer to know what is likely to be the energy generation performance of the proposed SPM when it is implemented [131, 136, 139, 141]. 
The hourly unmet users' load demands for all the zones are presented in Figures 76 to 81 . It is also obvious that the NE, NW and SW zones have higher initial scenario values compared to the NC, SE and SS zones. Therefore, the energy deficits for the NE, $\mathrm{NW}, \mathrm{NC}, \mathrm{SE}$, SS and SW zones for the initial scenario are 7,633 kWh/yr, 13,533 kWh/yr, 2,775 kWh/yr, 1,074 kWh/yr, 2,290 kWh/yr and 6,437 kWh/yr, respectively. These have been minimised in the scenarios 1 and 2 . The values for obtained for the scenario 1 are $278 \mathrm{kWh} / \mathrm{yr}, 803 \mathrm{kWh} / \mathrm{yr}, 204 \mathrm{kWh} / \mathrm{yr}, 739 \mathrm{kWh} / \mathrm{yr}, 1,166 \mathrm{kWh} / \mathrm{yr}$ and 1,033 kWh/yr, respectively, while those obtained for the scenario 2 are $543 \mathrm{kWh} / \mathrm{yr}, 609 \mathrm{kWh} / \mathrm{yr}, 444$ $\mathrm{kWh} / \mathrm{yr}, 464 \mathrm{kWh} / \mathrm{yr}, 1,170 \mathrm{kWh} / \mathrm{yr}$ and $895 \mathrm{kWh} / \mathrm{yr}$, respectively. The loss of energy probability ( $L O E P)$ for the initial scenario is $11.95 \%, 21.19 \%, 4.35 \%, 1.68 \%, 3.59 \%$ and $10.08 \%$, respectively. Also, the corresponding availability is $88.05 \%, 78.81 \%, 95.65 \%$, $98.32 \%, 96.41 \%$ and $89.92 \%$.

The LOEP for the scenario 1 is $0.43 \%, 1.26 \%, 1.07 \%, 1.16 \%, 1.82 \%$ and $1.62 \%$, respectively, with the corresponding availability of $99.57 \%, 98.74 \%, 98.93 \%, 98.84 \%$, $98.18 \%$ and $98.38 \%$. In addition, the LOEP for the scenario 2 is $0.99 \%, 1.27 \%, 0.73 \%$, $0.74 \%, 1.91 \%$ and $1.65 \%$, respectively, while the corresponding availability is $99.01 \%$, $98.73 \%, 99.27 \%, 99.26 \%, 98.09 \%$ and $98.35 \%$. These results show that the SPM models considered in the scenarios 1 and 2, can guarantee electricity supply to the intended users for $>98 \%$ of the time. This is based on the minimum SoC set point of $30 \%$ and the northern zones (NE, NW and NC) are found to have a relatively higher availability (see Table 14). 


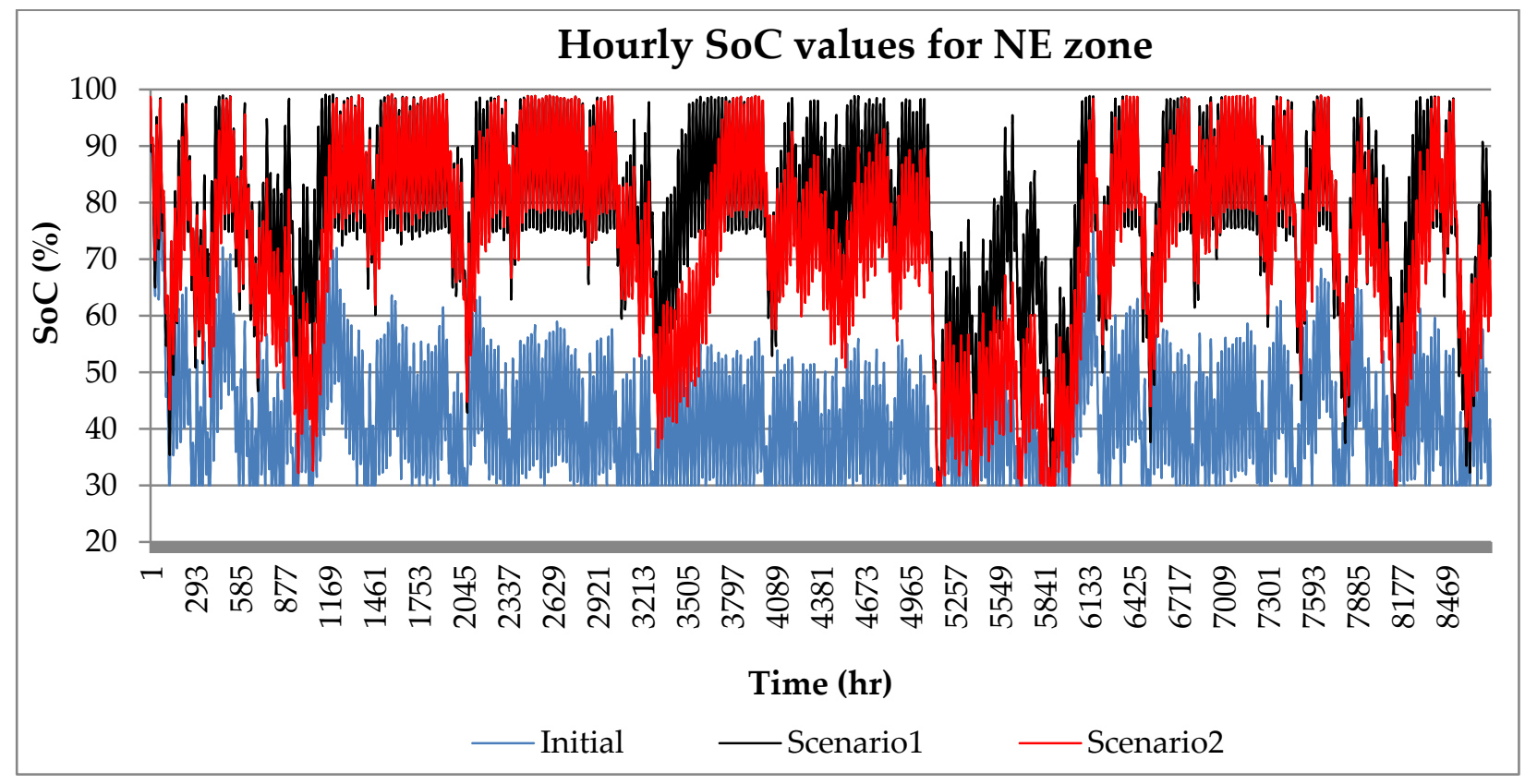

Figure 70: Hourly SoC values for the NE zone

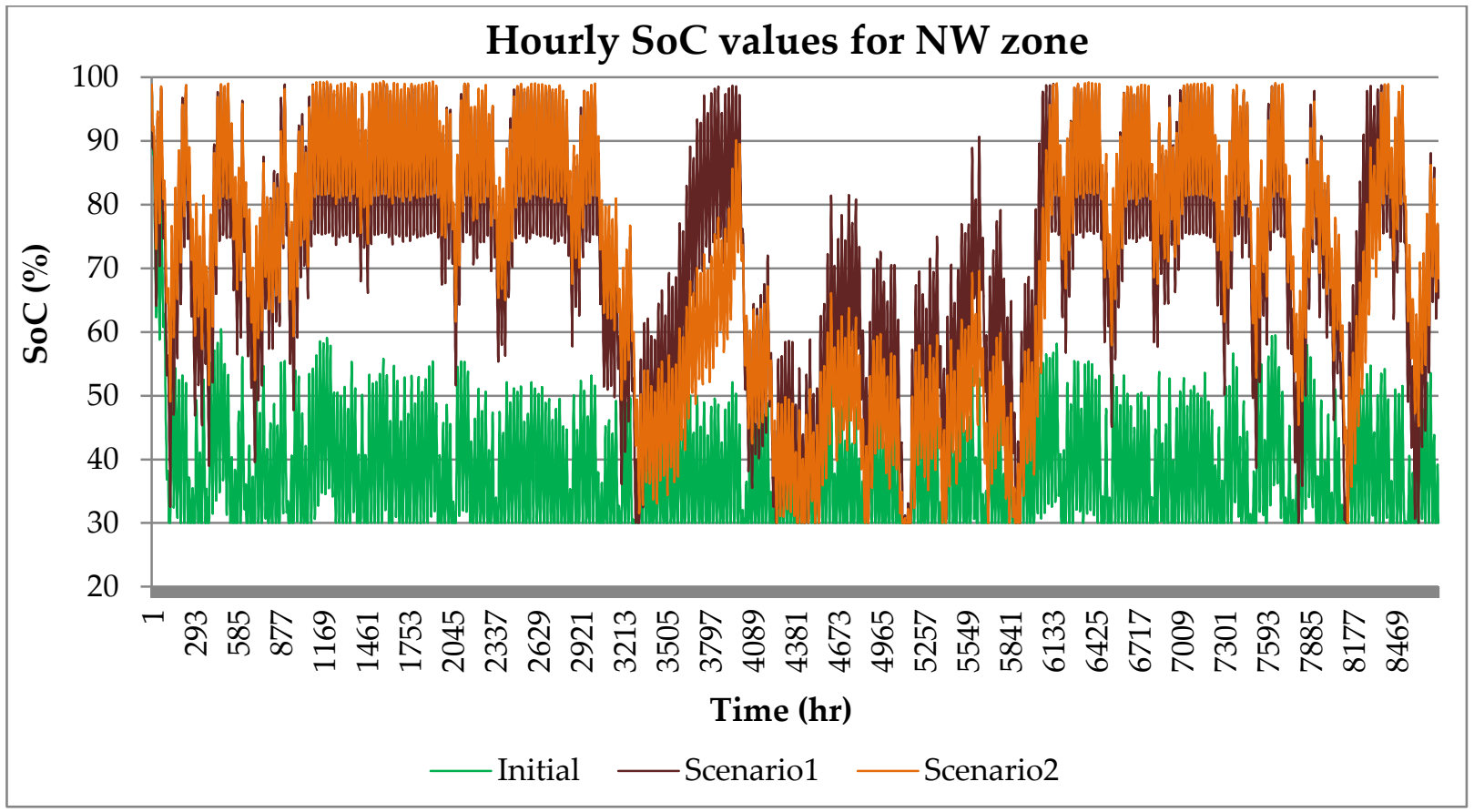

Figure 71: Hourly SoC values for the NW zone 


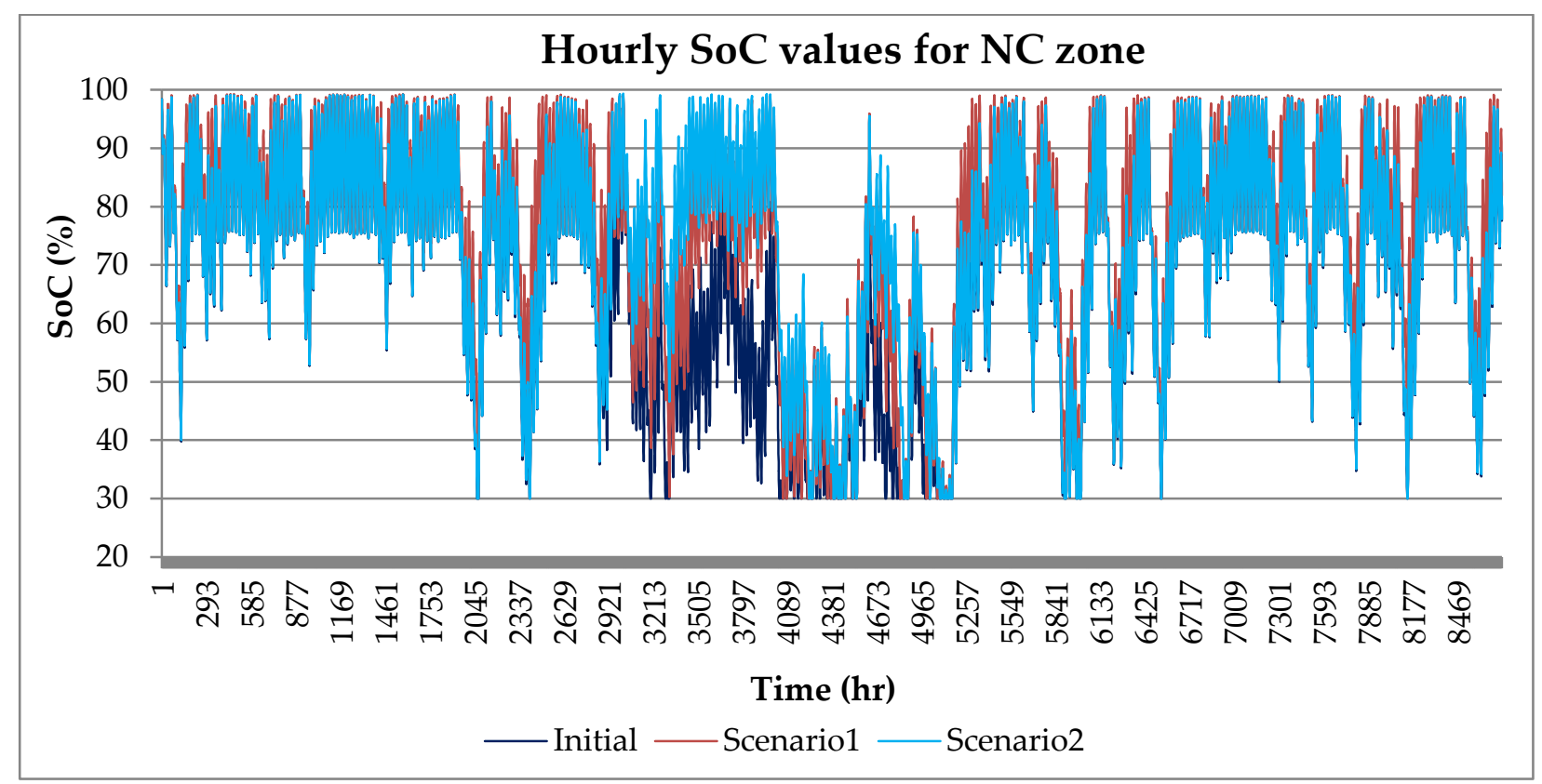

Figure 72: Hourly SoC values for the NC zone

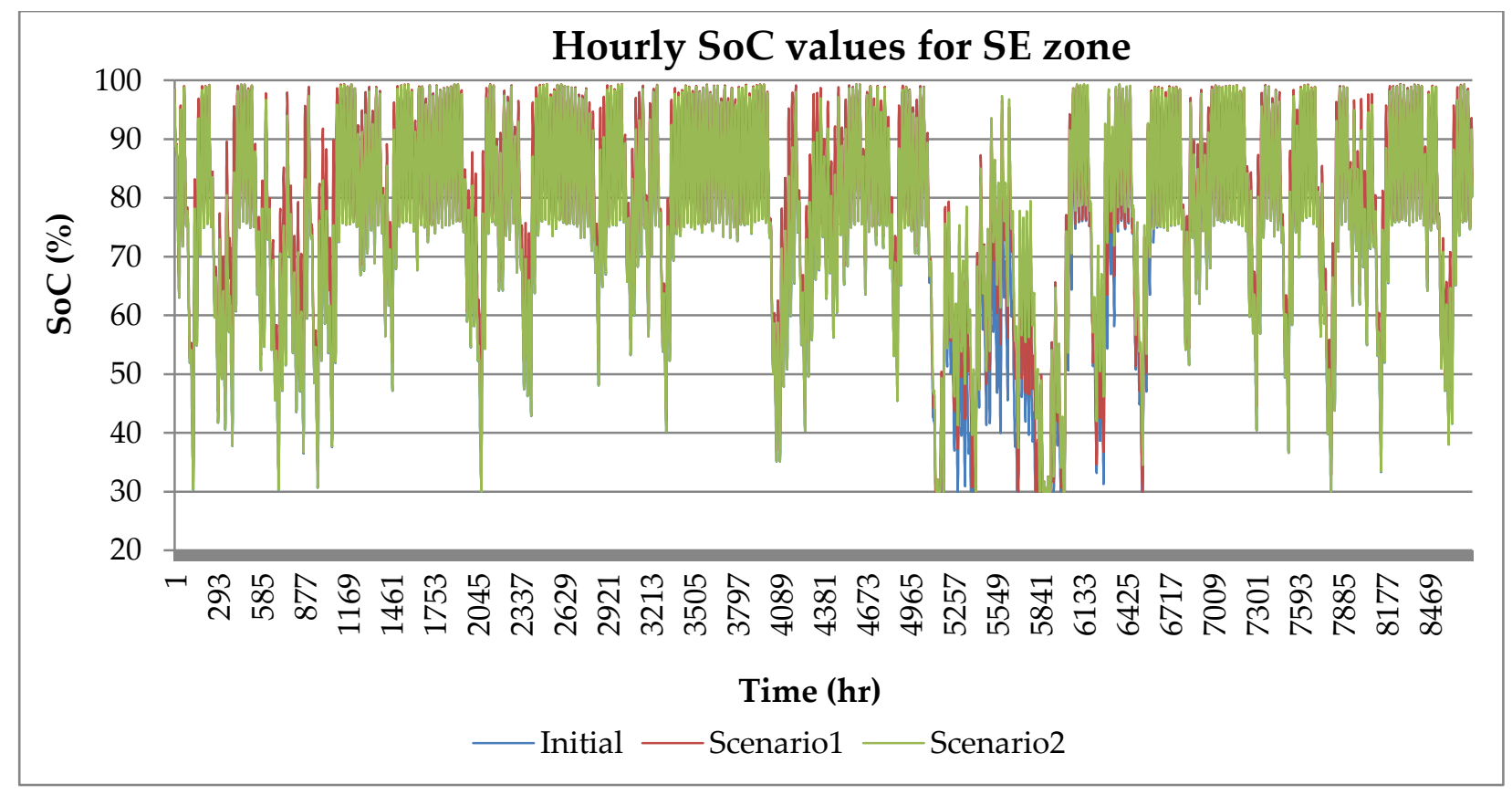

Figure 73: Hourly SoC values for the SE zone 


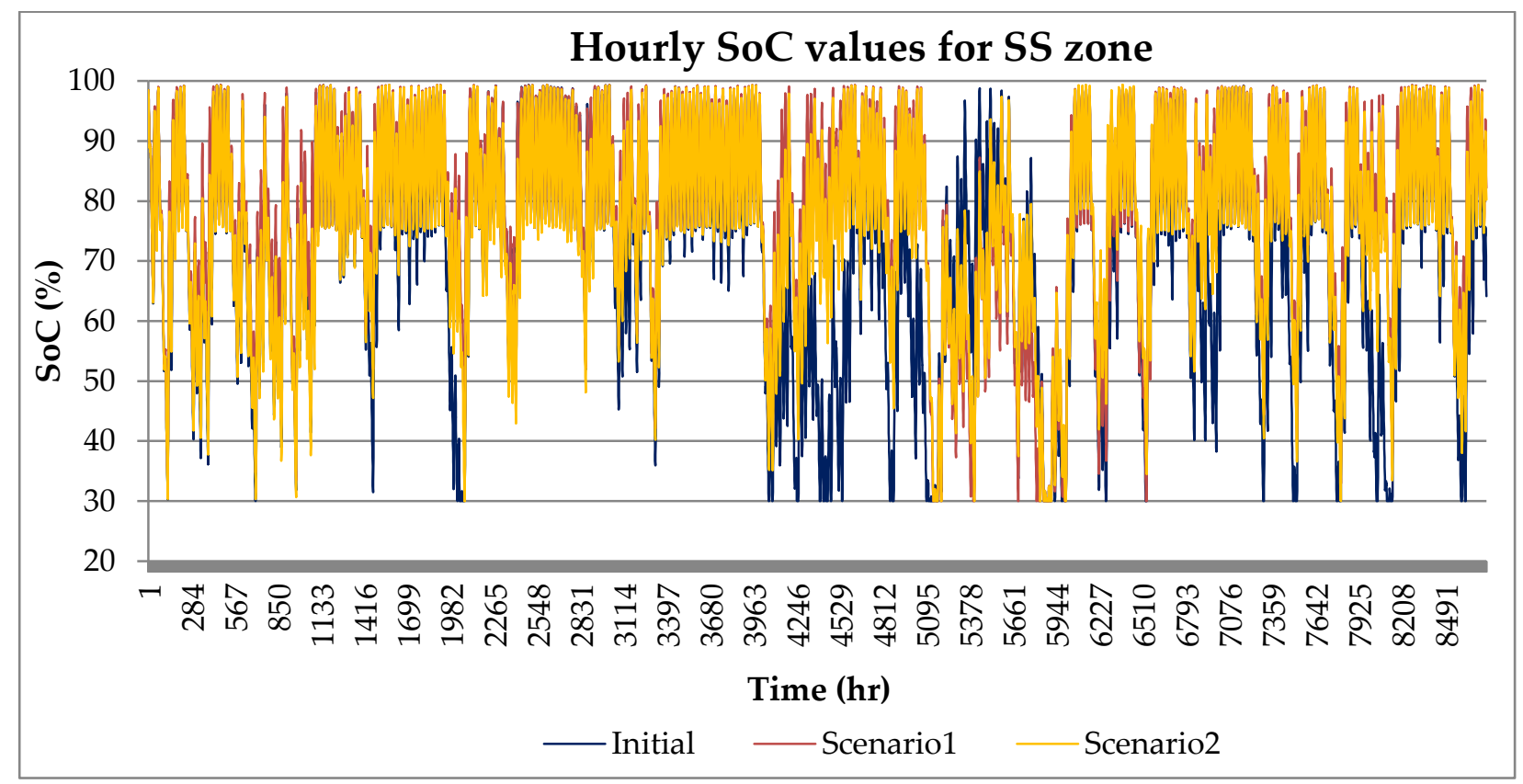

Figure 74: Hourly SoC values for the SS zone

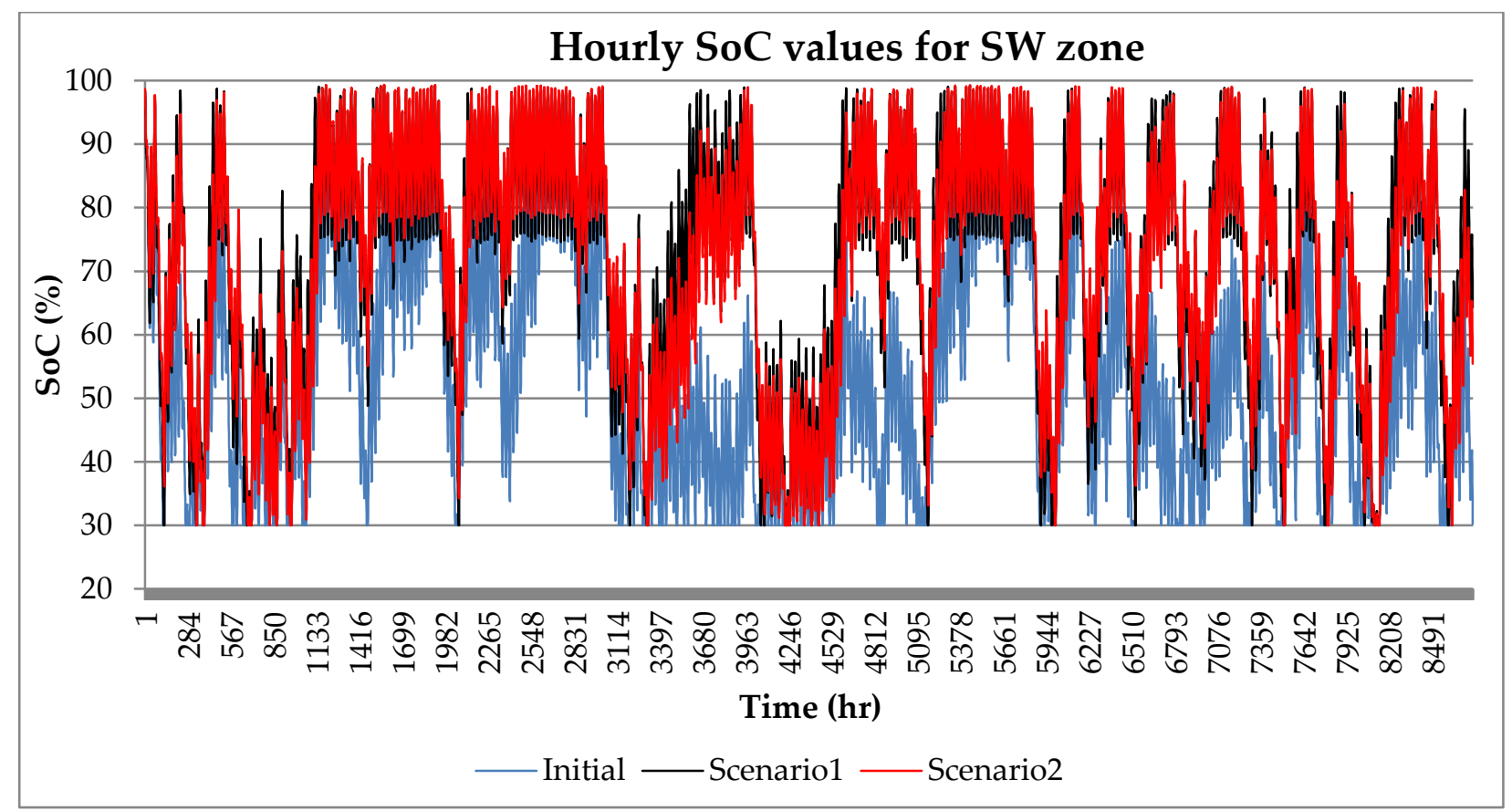

Figure 75: Hourly SoC values for the SW zone 


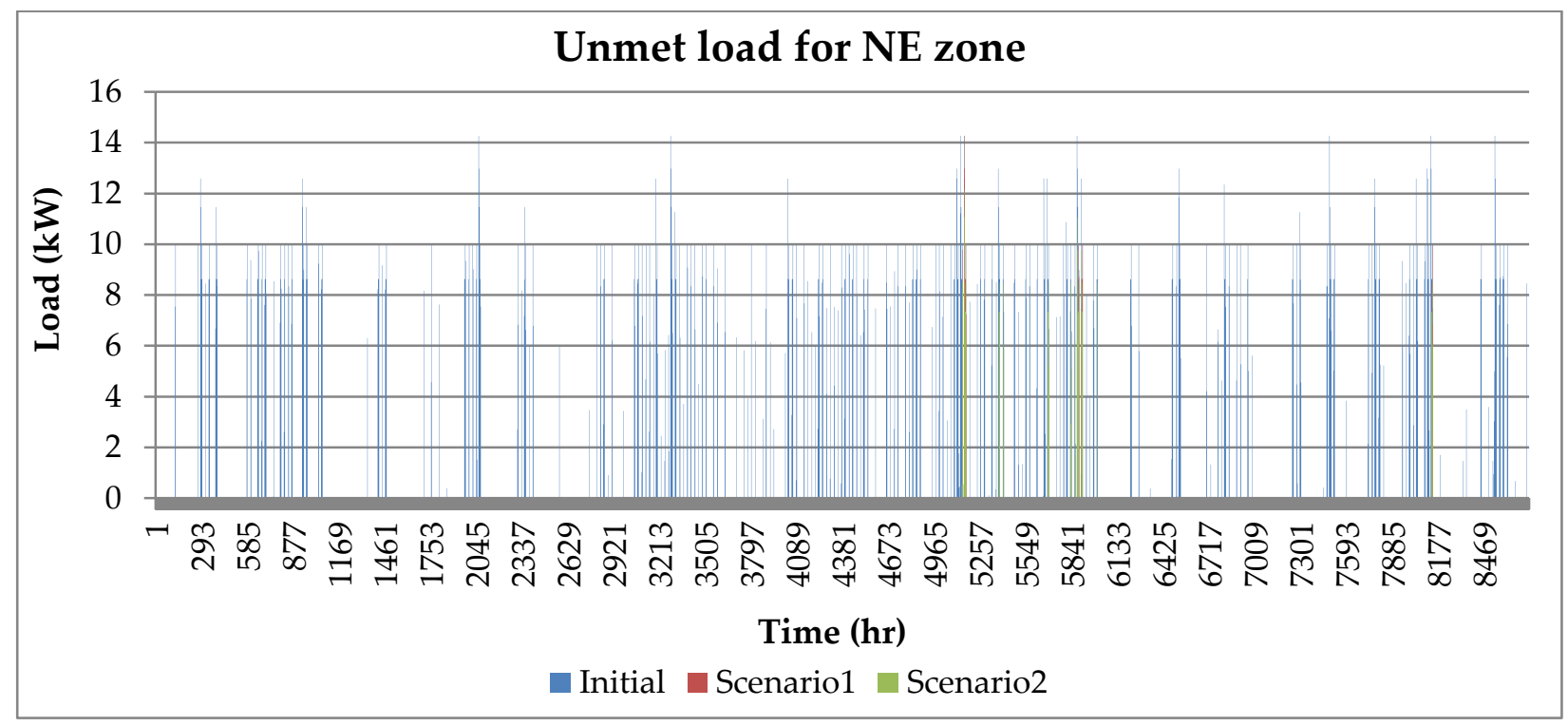

Figure 76: Unmet load demand for the NE zone

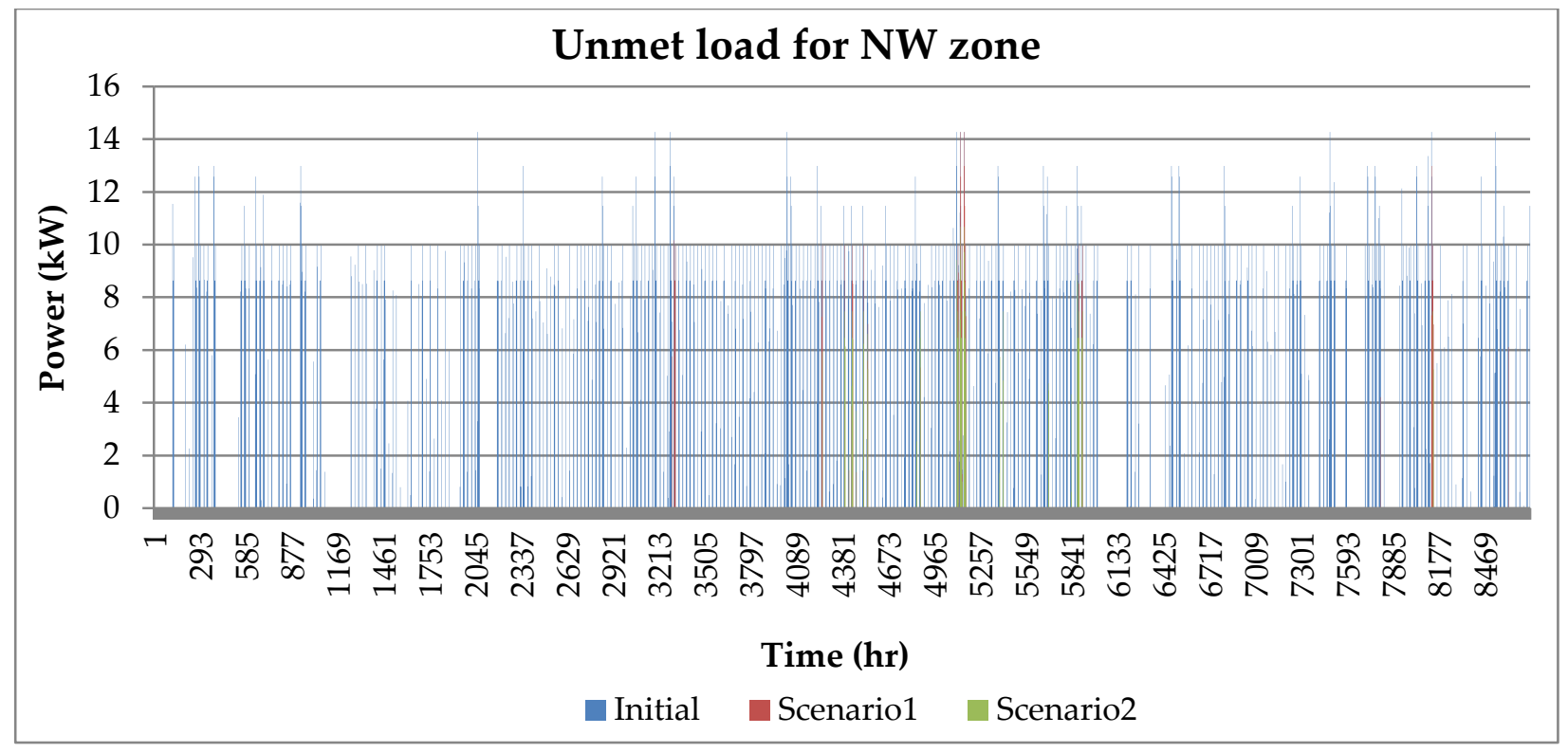

Figure 77: Unmet load demand for the NW zone 


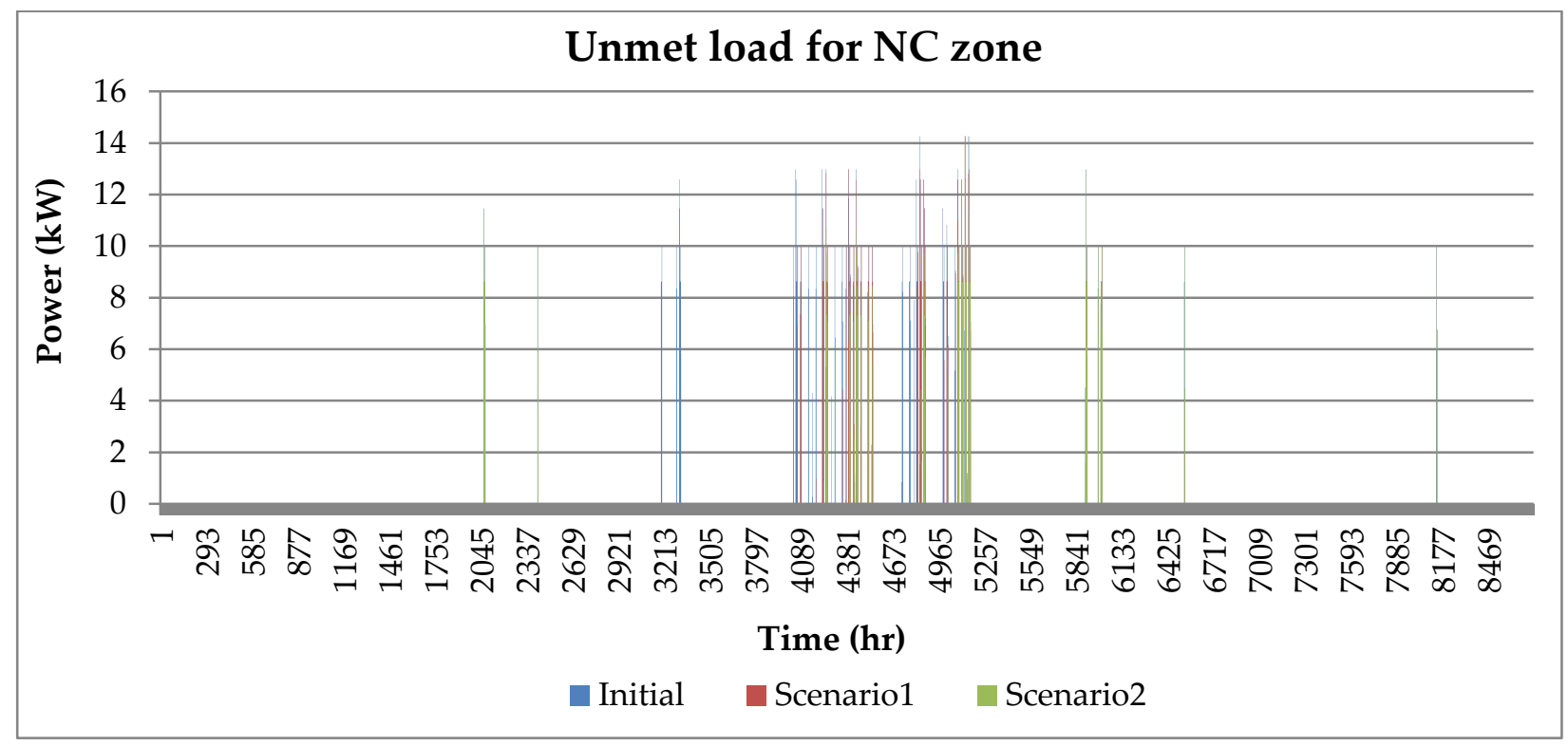

Figure 78: Unmet load demand for the NC zone

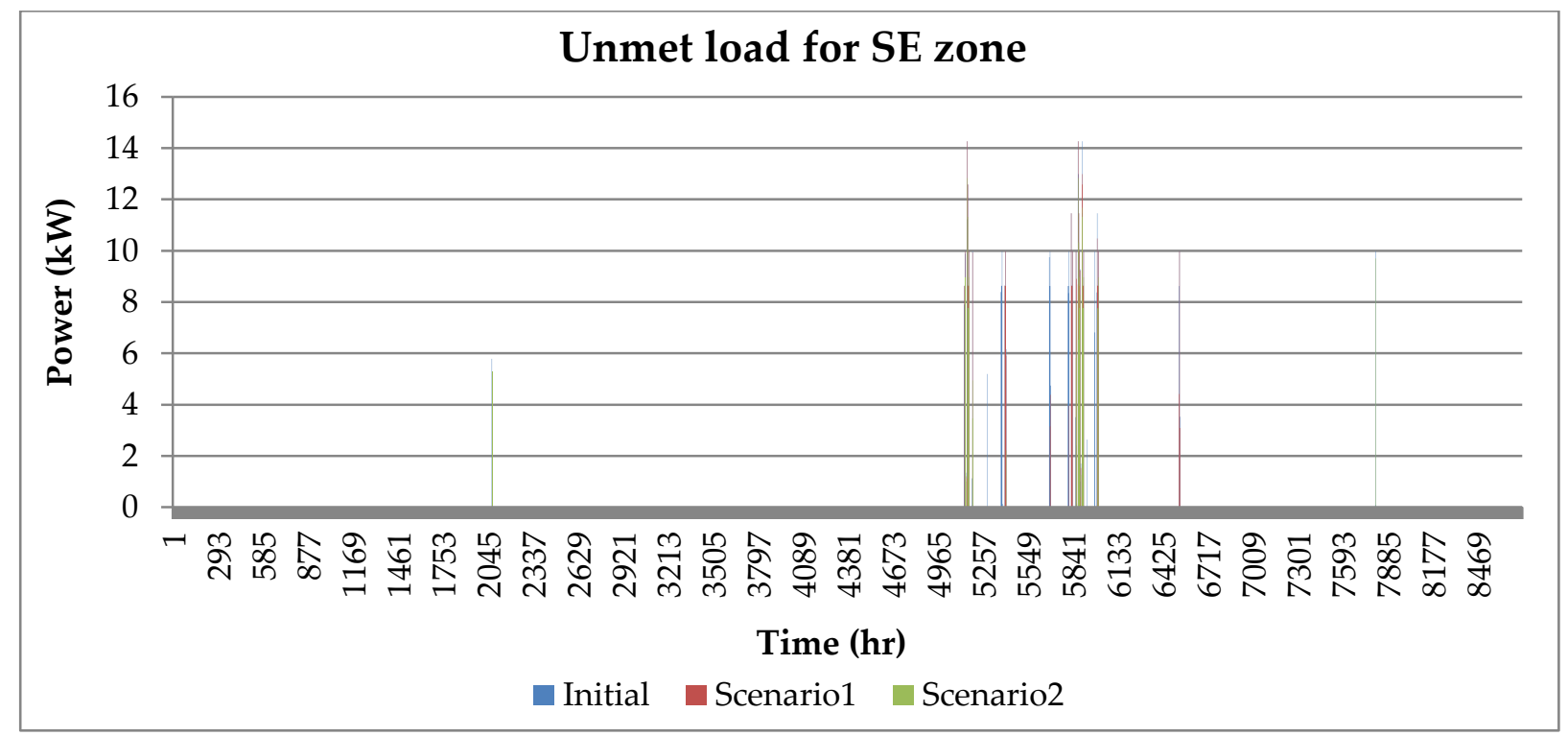

Figure 79: Unmet load demand for the SE zone 


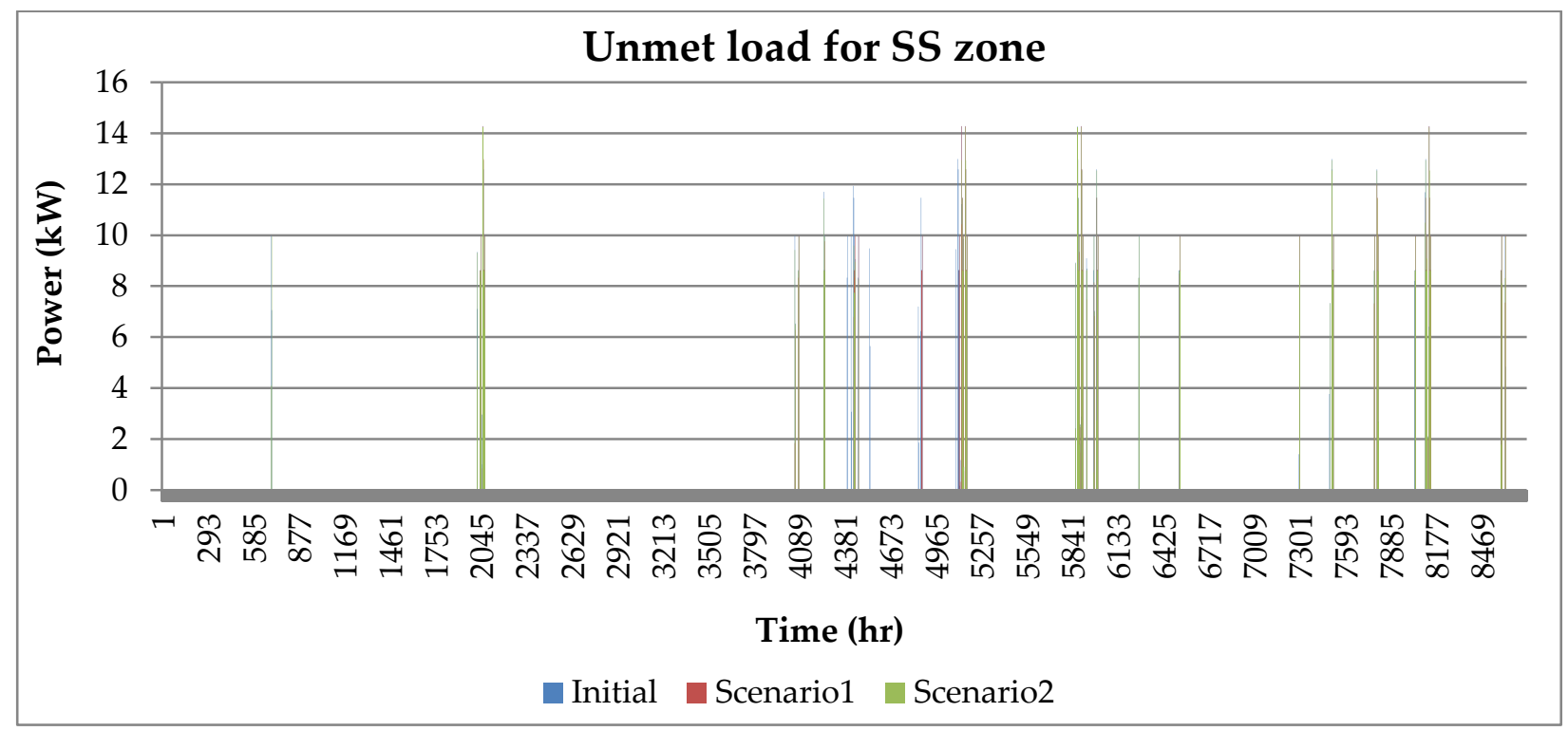

Figure 80: Unmet load demand for the SS zone

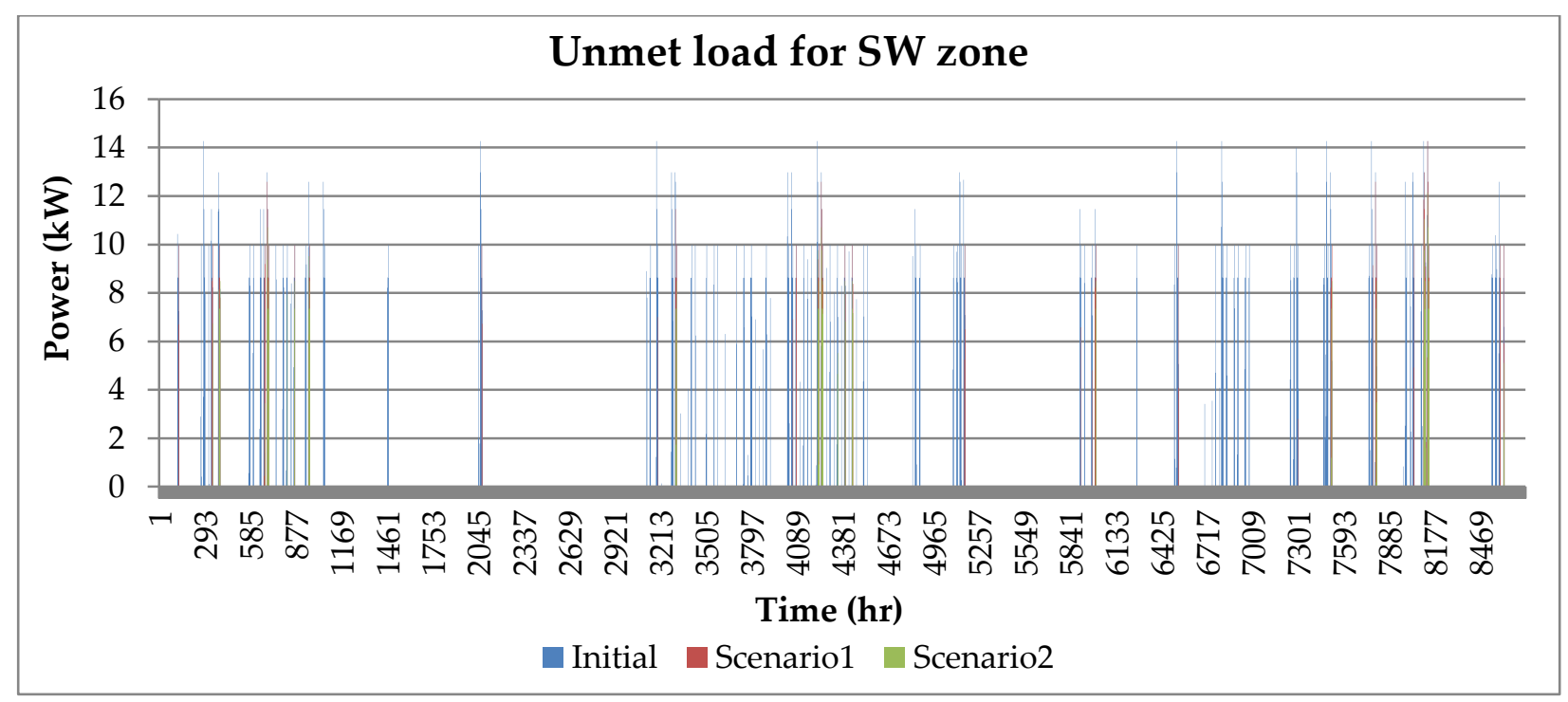

Figure 81: Unmet load demand for the SW zone

Table 14: Reliability indices for the different systems

\begin{tabular}{c|ccc|ccc|ccc}
\hline \multirow{2}{*}{ Location } & \multicolumn{2}{|c|}{ Unmet energy (kWh/yr) } & \multicolumn{3}{|c|}{ LOEP (\%) } & \multicolumn{3}{c}{ Availability (\%) } \\
\cline { 2 - 9 } & Initial & Scen. 1 & Scen. 2 & Initial & Scen. 1 & Scen. 2 & Initial & Scen. 1 & Scen. 2 \\
\hline $\mathrm{NE}$ & 7,633 & 278 & 543 & 11.95 & 0.43 & 0.99 & 88.05 & 99.57 & 99.01 \\
\hline
\end{tabular}




\begin{tabular}{c|ccc|ccc|ccc} 
NW & 13,533 & 803 & 609 & 21.19 & 1.26 & 1.27 & 78.81 & 98.74 & 98.73 \\
NC & 2,775 & 684 & 444 & 4.35 & 1.07 & 0.73 & 95.65 & 98.93 & 99.27 \\
SE & 1,074 & 739 & 464 & 1.68 & 1.16 & 0.74 & 98.32 & 98.94 & 99.26 \\
SS & 2,290 & 1,166 & 1,170 & 3.59 & 1.82 & 1.91 & 96.41 & 98.18 & 98.09 \\
SW & 6,437 & 1,033 & 895 & 10.08 & 1.62 & 1.65 & 89.92 & 98.38 & 98.35 \\
\hline
\end{tabular}

The battery constraints and the average SoC analysis results reported in this thesis can be compared with those reported in the literature [180, 185, 187, 188]. For instance, in [180], about $88 \%$ of the battery SoC values of a stand-alone PV system for a remote island in Hong Kong, is above the SoC of $50 \%$ and $45 \%$. Also, about $1 \%$ of the SoC values are at the minimum set point of $20 \%$.

The energy system analysis in [185] was based on a minimum battery set point of $40 \%$ and this level was reached during the months with the highest energy demand. It is obvious from the results that the mean $\mathrm{SoC}$ values for the scenarios 1 and 2 have a higher probability for SoC $>30 \%$ compared to the initial scenario.

However, this study has carefully considered the intermittent solar energy resources of different locations. This is proven by the results obtained for the hourly battery SoC variations over the year. The approach considers the hourly battery energy profiles and the periods when a loss of energy is likely to be experienced based on the set point of $30 \%$. Such an approach deepens the PV knowledge compared to some existing research studies reported in $[180,185,186]$. The hourly battery SoC variations were not discussed in those studies. The proposed SPM's loss of energy probability and the availability have also been carefully estimated. An $L O E P$ of $0 \%$ means that the users' load demand will always be met, while a $100 \%$ value indicates that the users' energy demand will never be met $[158,159]$.

Furthermore, the thesis results are comparable to a number of previous studies such as those reported in [168], [187] and [188]. These also include the battery analysis on an hourly basis. The SoC and the loss of load were also reported in [159]. The authors in [168] present the hourly battery SoC values for the whole year and for a 
week, which are within the battery constraints (i.e. SoC of 35\%). The SoC values in [187] for 8760 -hr are also found to be above the set point of $20 \%$.

The SoC values for a 4000-hour operation in [187] has also been reported to be above the minimum value of $20 \%$. The loss of power supply probability (LPSP) of $2.7 \%$ is reported for the energy model in [159]; the LPSP values of $5 \%$ and $4 \%$ were also reported in [168] for the traditional and the proposed energy models, respectively. These studies report the availability of $97.3 \%, 95 \%$ and $96 \%$, respectively, which are also comparable to the results presented in Table 14.

The loss of load probability ( $L L P$ ) of $0.53 \%$ and $0.54 \%$ have also been reported in [187] for priority loads, while the LLPS of $25.87 \%$ and $26.29 \%$ were reported for nonpriority loads. These have the corresponding availability of $99.47 \%$ and $99.46 \%$, and $74.13 \%$ and $73.71 \%$. The loss of load hours of 20, 24, 6, 5, 20, 57, 66, 61, 39, 30, 25 and 21 has been reported in [191] for a PV-based energy system in India, respectively, for January to December.

This thesis has carefully considered the identified knowledge gaps in [103-126, 128]. It has addressed the issues by introducing detailed battery energy analysis, including the evaluation of unmet energy, loss of energy probability and the availability of the proposed SPM systems, using Nigeria's six geo-political zones as case studies.

\subsection{Design safety factor}

The SPM's design safety factors are shown in Table 15. The "Original" case signifies the ideal SPM size resulting from Eq. (15) with a $d_{s f}$ of 1.0. This capacity is not adequate for supporting the users' load requirements because of the effect of losses. The "Initial" scenario is obtained with a $d_{s f}$ of 1.2 and it serves as the baseline (see Chapter 3). The $20 \%$ additional size in the initial scenario stems from the general guidelines specified in [131]. Since this is a general recommendation, it is also necessary to investigate carefully the system's adequacy for the intended applications.

The "Scen.1" is the scenario with the increased SPM capacity and the values obtained for the NE, NW, NC, SE, SS and SW zones are 1.50, 1.64, 1.33, 1.28, 1.24 and 1.44 , respectively. The analysis clearly shows that though the southern region has larger SPM capacities, it requires relatively lower design safety factors. In the case of the northern region, relatively smaller SPM sizes result in higher design safety factors. The disparity in the solar irradiance and the ambient temperature of these regions is 
responsible for such trends (see Figures 13 to 16). Therefore, an SPM system designed for the northern region, for instance, will not perform well in the southern region even with the same load demand requirements, and vice versa.

The evaluation results comply with the established photovoltaic system convention that the environmental factors of a location are a crucial determinant of the solar PV design and performance [18, 19]. The "Scen.2" is the scenario with reduced users' load demands and has the same $d_{s f}$ with the initial scenario. The minimum load demand reduction of $15 \%, 25 \%$ and $10 \%$ are considered for all the months for the systems in the NE, NW and SW zones, respectively. Also, a 15\% load reduction is considered for May, June and July for the system in the NC zone; a 15\% load reduction is also considered for August and September for the system in the SE zone, while a 10\% load reduction is used for July for the system in the SS zone.

The design safety factors presented in Table 15 can serve as benchmarks for estimating the solar photovoltaic microgrid capacities in the specified locations, based on the scenarios 1 and 2. This will be useful for determining the minimum SPM capacity that will support the users' maximum daily electricity demand, while also taking the possible energy losses into consideration.

Table 15: Design safety factor for the zones

\begin{tabular}{c|ccc|c|cccc}
\hline Location & \multicolumn{3}{|c|}{ SPM Capacity (kW) } & \multicolumn{4}{c}{ Design Safety Factor $\left(d_{s f}\right)$} \\
\cline { 2 - 9 } & Original & Initial & Scen.1 & Scen.2 & Original & Initial & Scen.1 & Scen.2 \\
\hline NE & 33.39 & 40.07 & 50 & 40.07 & 1.0 & 1.2 & 1.50 & 1.2 \\
NW & 33.58 & 40.30 & 55 & 40.3 & 1.0 & 1.2 & 1.64 & 1.2 \\
NC & 45.13 & 54.15 & 60 & 54.15 & 1.0 & 1.2 & 1.33 & 1.2 \\
SE & 50.72 & 60.86 & 65 & 60.86 & 1.0 & 1.2 & 1.28 & 1.2 \\
SS & 50.73 & 60.87 & 63 & 60.87 & 1.0 & 1.2 & 1.24 & 1.2 \\
SW & 38.88 & 46.66 & 56 & 46.66 & 1.0 & 1.2 & 1.44 & 1.2 \\
\hline
\end{tabular}


The relationship between different battery sizes and the depth of discharge (DoD) is presented in Figure 82. Basically, the battery energy capacity reduces as the DoD increases. The battery bank in this thesis is based on a DoD of $50 \%$, and with this size, it is expected that the battery will have a long service life. The relationship between the battery DoD and the cycle life is also shown in Table 16 [7].

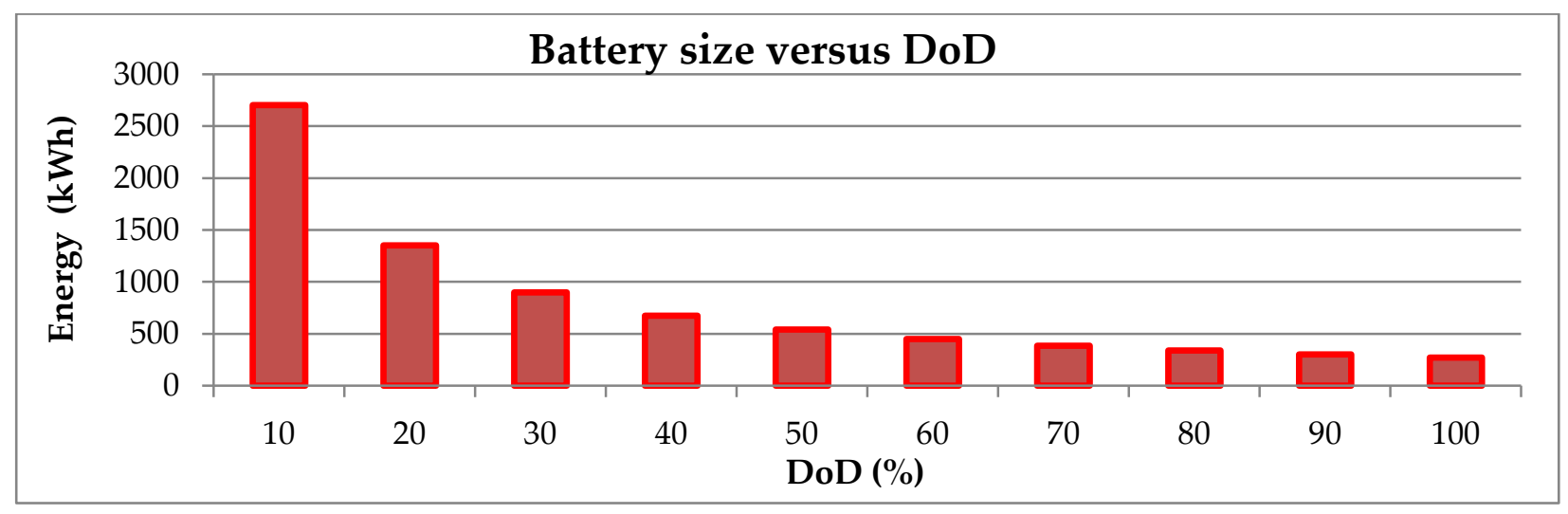

Figure 82: Battery capacity and the corresponding DoD

Table 16: Battery DoD versus lifespan

\begin{tabular}{ccc}
\hline DoD (\%) & Cycle life & Life span (yr) \\
\hline 100 & 600 & 1.64 \\
80 & 830 & 2.27 \\
70 & 870 & 2.8 \\
50 & 1650 & 4.52 \\
\hline
\end{tabular}

\subsection{Chapter summary}

The chapter has presented the scenarios, detailed results, and analyses of the proposed solar photovoltaic microgrid systems with no load demand growth. These involved sizing the SPM's main components, considering the design parameters and constraints that have been earlier discussed in Chapters 3 and 4 . In addition, a comprehensive assessment of the system's power and energy outputs, energy losses and efficiency, battery state of charge, unmet load, loss of energy probability and the availability was done. The results and analysis were presented for three different scenarios, such as the initial scenario, scenario 1 and the scenario 2 . The initial scenario represents the baseline, while the scenario 1 focuses on the increase in the SPM capacities. The scenario 2 considers the users' load demand reduction. 
The chapter has also demonstrated that an analysis of losses is a very important aspect of the SPM's design and planning, which should not be overlooked or underestimated. The SPM's losses have been carefully considered in determining the appropriate system sizes and performance prediction. The SPM capacities range from 40.07 to $65 \mathrm{~kW}$ for the three scenarios. There are disparities in the losses due to the locations' different ambient temperature values. Also, the losses due to temperature for the northern region, i.e. NE, NW and NC zones are $20.35 \%, 19.06 \%$, and $20.45 \%$, respectively, of the baseline energy value. However, the values obtained for the southern region, i.e. SE, SS and SW zones are less than $20 \%$ of the baseline value.

The results for the SPM's yields and losses have also provided a performance summary that can be compared with PV systems in the other locations. The yields for the zones range from 3.26 to $4.72 \mathrm{hr} / \mathrm{d}$, while the losses range from 0.84 to $1.37 \mathrm{hr} / \mathrm{d}$. The SPM's average efficiencies for the zones also range from 13.03 to 13.95 .

The chapter also introduced a design safety factor $\left(d_{s f}\right)$ for determining suitable photovoltaic microgrids for different locations. The benchmark $d_{s f}$ for the NE, NW, NC, SE, SS and SW zones is 1.5, 1.64, 1.33, 1.28, 1.24 and 1.44, respectively. Once these factors have been calculated, they can be used to determine system sizes for the specified locations. The result clearly shows that the location having the lowest solar energy resource, i.e. SS zone has the least $d_{s f}$. This is because it has the lowest temperature losses and the highest efficiency compared to the other zones. The SPM sizes range from 40.07 to $65 \mathrm{~kW}$ for the considered scenarios.

The proposed SPM's reliability has also been examined and the results show that the system's availability ranges from 78.81 to $89.92 \%, 98.18$ to $99.57 \%$ and 98.35 to $99.27 \%$ for the initial scenario, scenario 1 and scenario 2, respectively. This clearly demonstrates that the scenarios 1 and 2 have a better performance than the initial scenario, through SPM's capacity increase and users' load reduction. The next chapter discusses the results and analyses of the SPM systems with users' load growth. 


\section{Chapter 6}

\section{Microgrid analysis with load growth}

This chapter presents the scenarios, detailed results and analyses of the proposed solar photovoltaic microgrid systems with load demand growth. It begins with ascertaining the impact of the load growth on the existing system capacities. This is considered in terms of the battery state of charge, unmet load, loss of energy probability and availability parameters. The assessment results are then employed to make technical decisions in situations when the existing SPM capacities are not able to support the increased users' demands.

The chapter is divided into four parts. The first presents the results and analyses of the initial system capacities with load demand growth. The second aspect provides the assessments of new SPM capacities with the initial battery bank, while the third section focuses on the analyses of new SPM capacities with the new battery bank. The fourth part highlights the relationship of the load growth factor with the design safety factor and the ideal SPM capacities. A summary concludes the chapter.

\subsection{Initial system capacity and performance}

\subsubsection{Hourly SoC analysis}

The load growth of $50 \%$ is first considered for the proposed SPMs. This represents $50 \%$ load demand increase above the baseline demand of $175 \mathrm{kWh} / \mathrm{d}$. In a practical sense, the information about the users' load growth is not usually available, but the load demand increase values have been introduced for the purpose of analysis. The load growth factors $\left(l_{q f}\right)$ range from 1.042 to 1.500 (see Table 17) [158].

A $50 \%$ load growth means that the initial annual demand of $63,875 \mathrm{kWh}$ (i.e. 175 $\mathrm{kWh}$ /day) is increased to $95,630 \mathrm{kWh}$ (i.e. $262 \mathrm{kWh} /$ day). The energy requirements of 63,875 and $95,630 \mathrm{kWh}$ stand for $100 \%$ and $150 \%$ users' demand (UD), respectively. The percentage load growth is also shown in Table 17. The 63,875 kWh represents the baseline demand for the 24 houses, while the demand of $95,630 \mathrm{kWh}$ corresponds to the total demand when the baseline demand increases by $50 \%$. The total demand when the users' consumption increases by 50\%, thus, translates an energy demand required by 36 
houses. The SPM size, SoC, unmet energy, LOEP and the availability for the reference demand, in this case, are based on scenario 1 that was previously discussed.

Furthermore, the hourly battery SoC variations are again used to investigate the effect of the load growths on the proposed SPM models. Figures 83 to 88 show the battery SoC with load growth. It is clearly seen that the SoC values decrease as the load demand increases, e.g. the least SoC values are obtained for the $125 \%$ UD compared to the others.

Table 17: Load growth scenarios

\begin{tabular}{ccccc}
\hline S/No. & $\begin{array}{c}\text { Demand } \\
\mathbf{( k W h / y r )}\end{array}$ & $\begin{array}{c}\text { Percentage load } \\
\text { growth (\%) }\end{array}$ & UD (\%) & $l_{8 f}$ \\
\hline 1 & $63,875^{*}$ & - & 100 & 1.000 \\
2 & 66,430 & 4.2 & 104.2 & 1.042 \\
3 & 69,350 & 8.3 & 108.3 & 1.083 \\
4 & 71,905 & 12.5 & 112.5 & 1.125 \\
5 & 74,460 & 16.7 & 116.7 & 1.167 \\
6 & 77,015 & 20.8 & 120.8 & 1.208 \\
7 & 79,935 & 25.0 & 125 & 1.250 \\
8 & 82,490 & 29.2 & 129.2 & 1.292 \\
9 & 85,045 & 33.3 & 133.3 & 1.333 \\
10 & 87,965 & 37.5 & 137.5 & 1.375 \\
11 & 90,520 & 41.7 & 141.7 & 1.417 \\
12 & 93,075 & 45.8 & 145.8 & 1.458 \\
13 & 95,630 & 50.0 & 150 & 1.500 \\
\hline
\end{tabular}




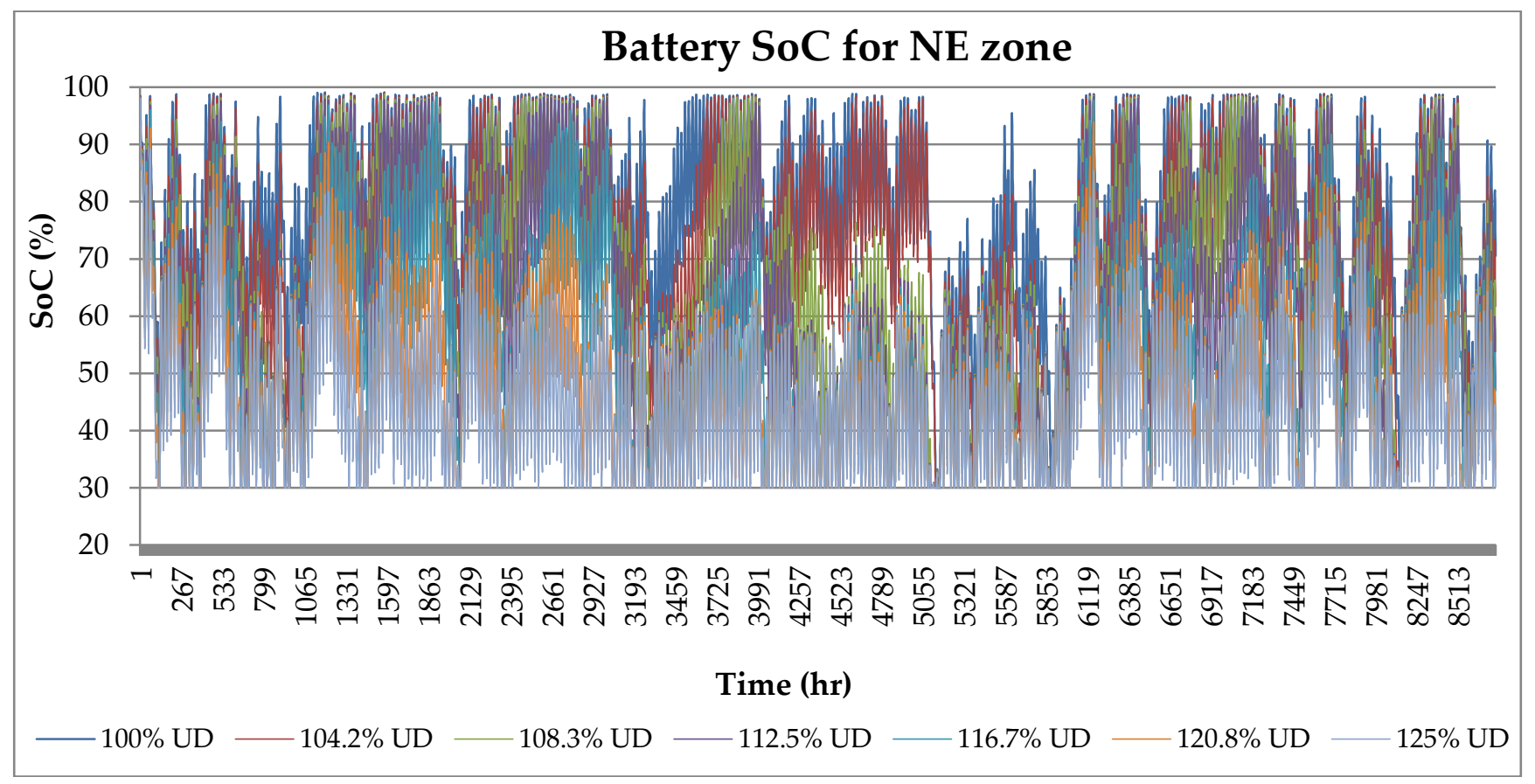

Figure 83: Battery SoC for load growth for the NE zone

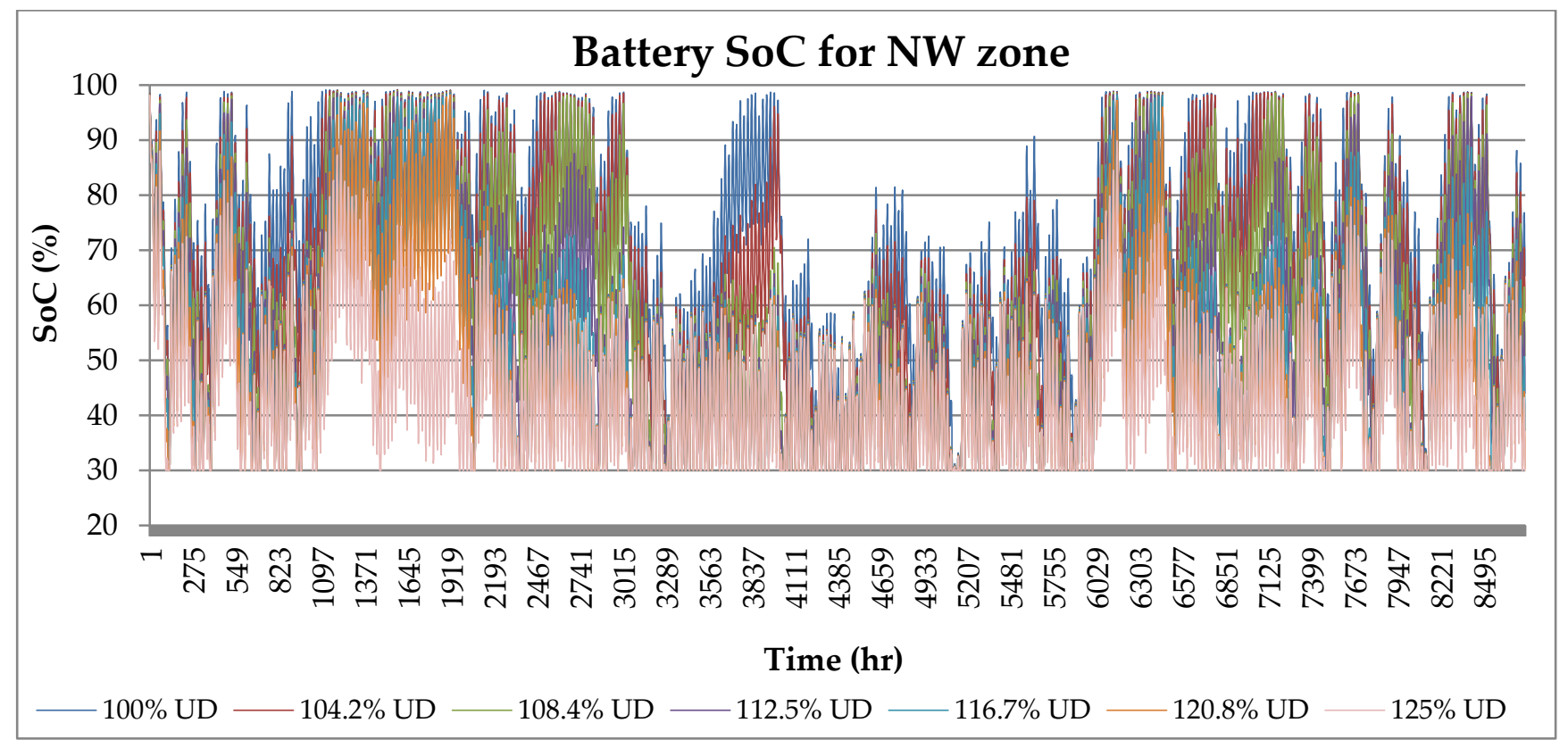

Figure 84: Battery SoC for load growth for the NW zone 


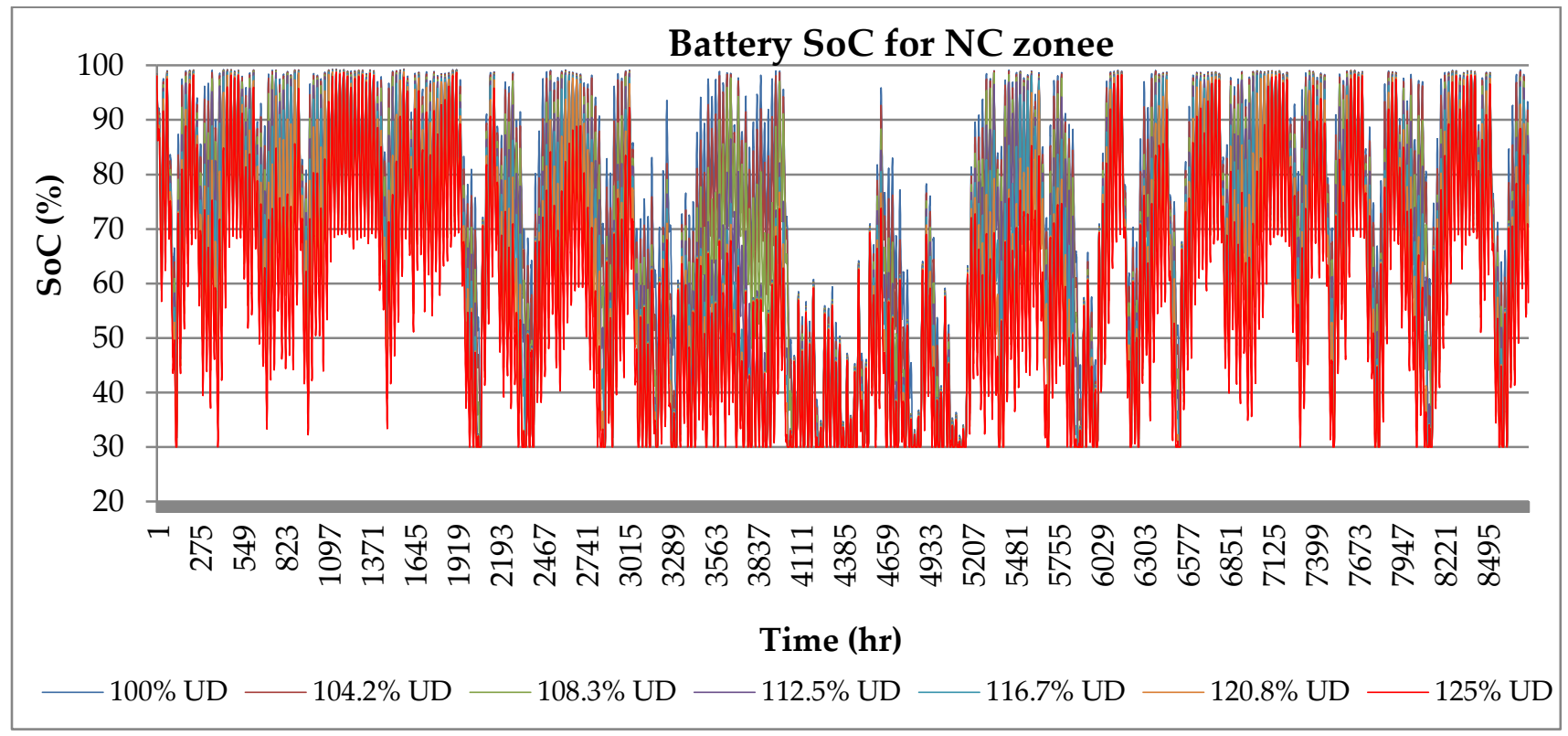

Figure 85: Battery SoC for load growth for the NC zone

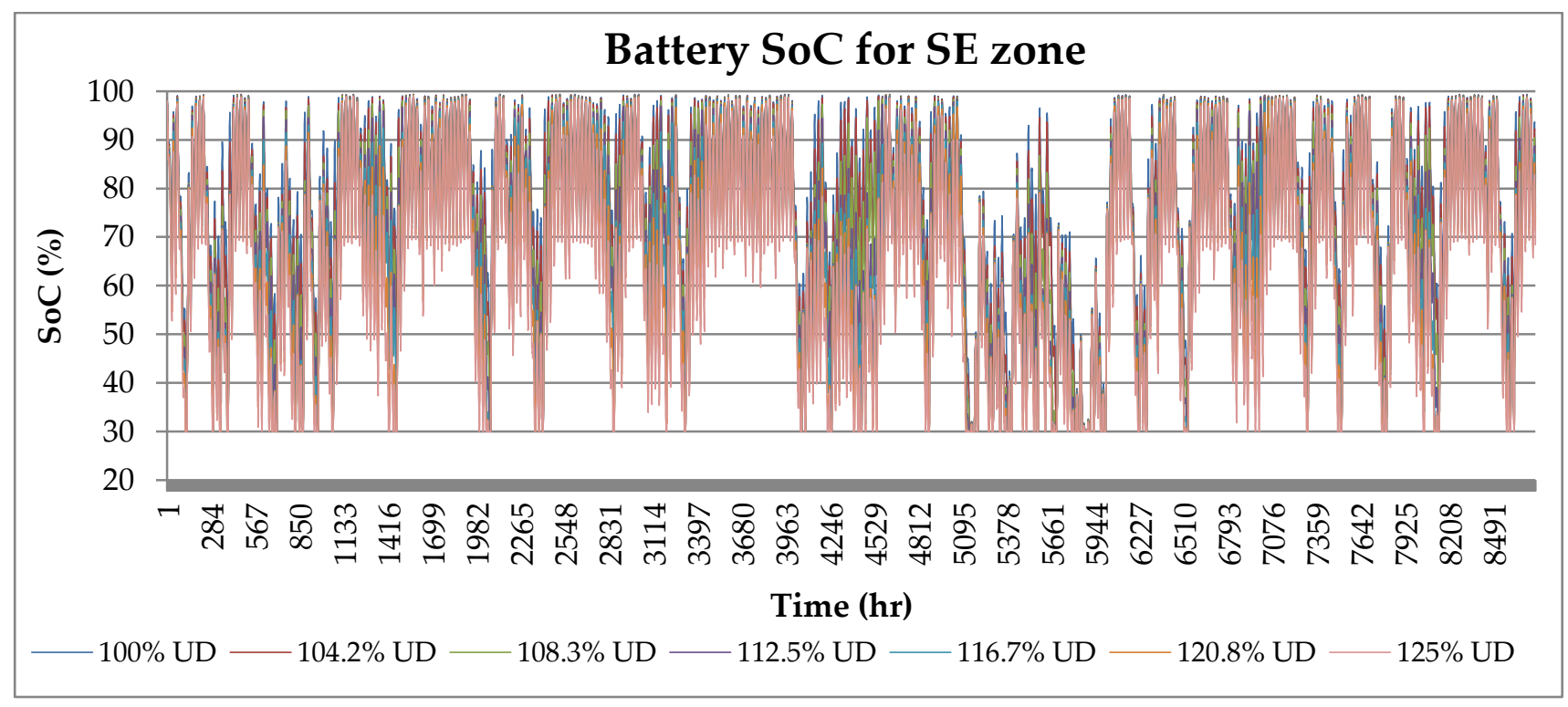

Figure 86: Battery SoC for load growth for the SE zone 


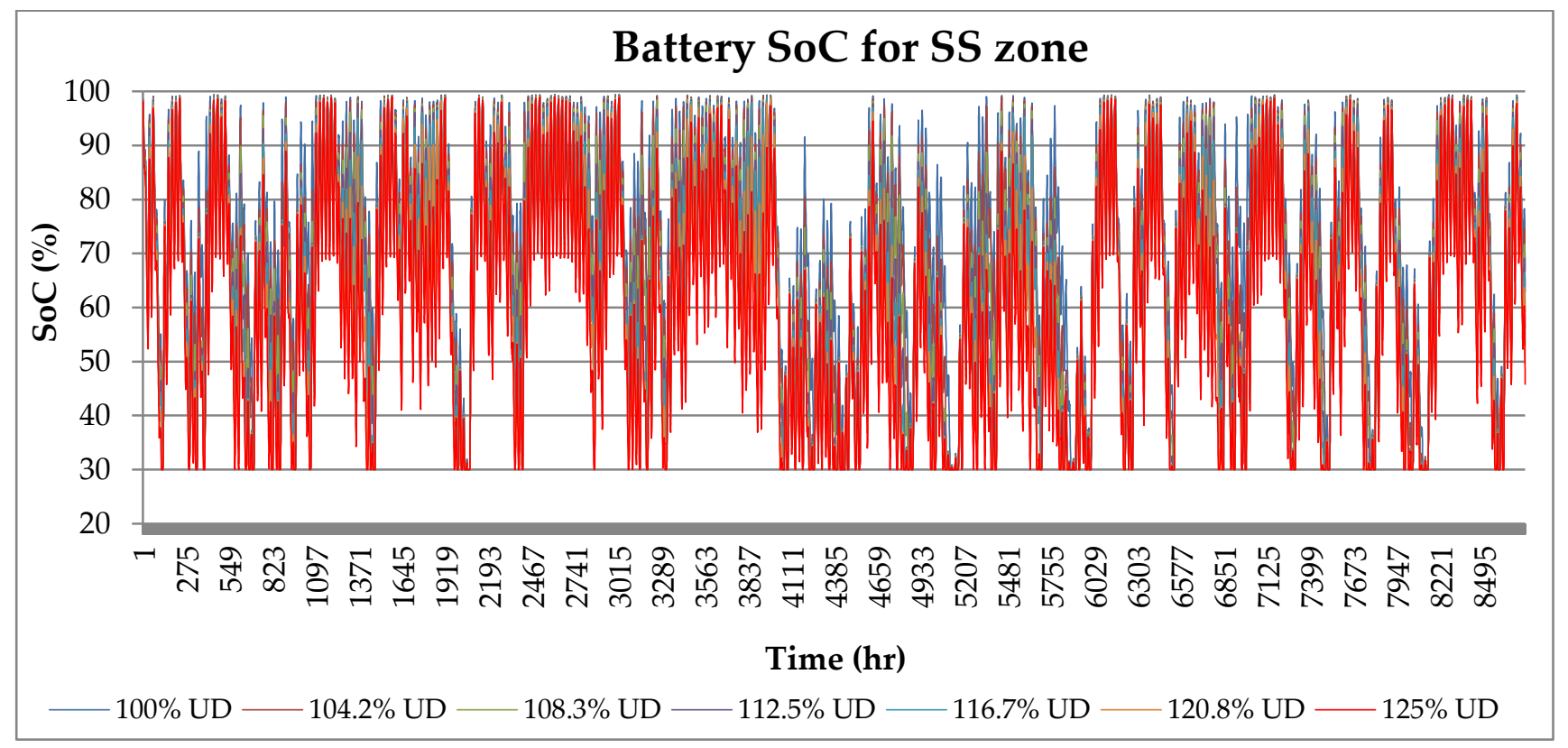

Figure 87: Battery SoC for load growth for the SS zone

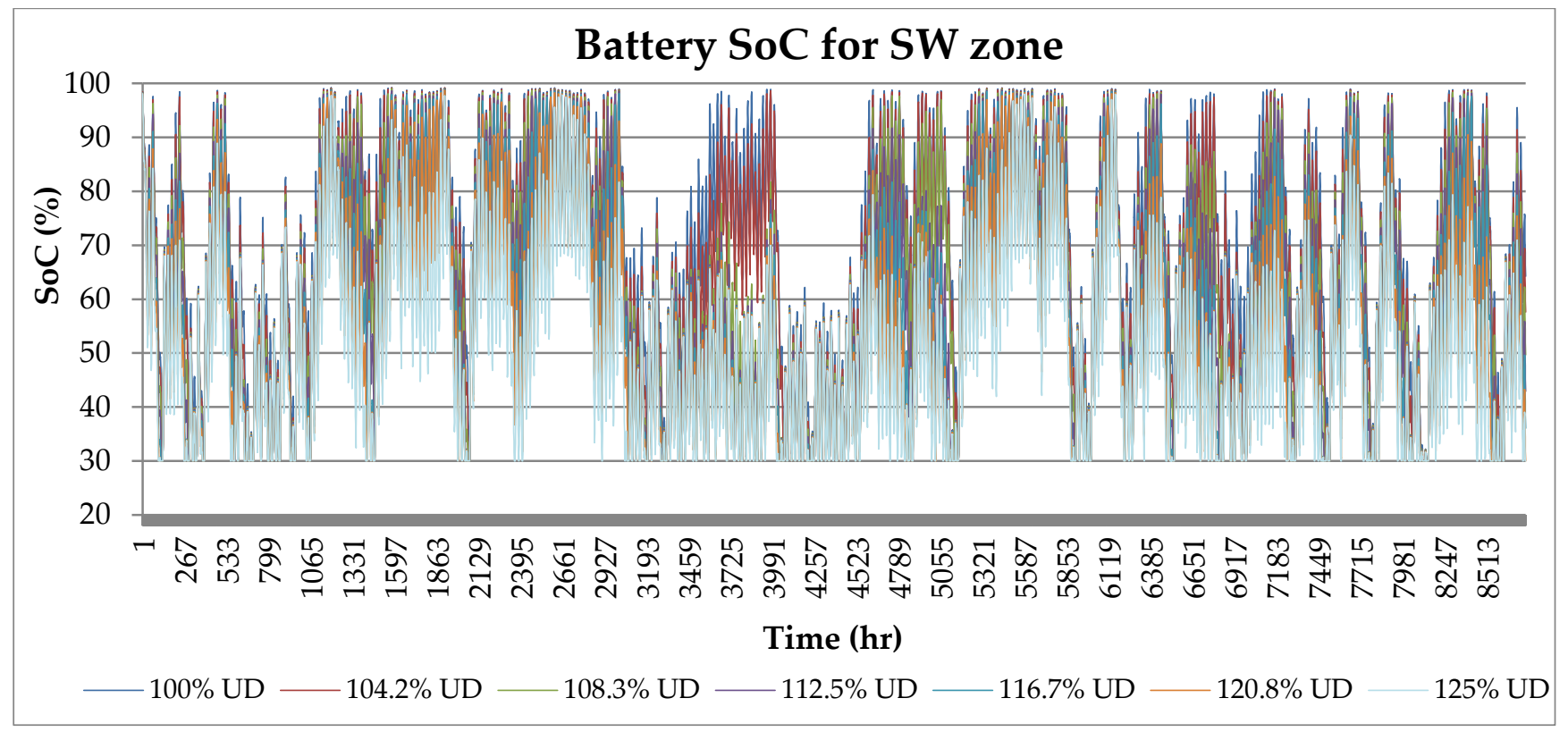

Figure 88: Battery SoC for load growth for the SW zone

\subsubsection{System reliability analysis}

The users' unmet load demands over the year are presented in Figure 89, while the loss of energy probability and the availability are shown in Figures 90 and 91 . While the unmet load demand and the LOEP increases, the availability decreases, indicating 
that the SPM's reliability reduces compared to the base case - scenario 1 . The minimum values of the unmet energy in Figure 89 are the same as those reported for scenario 1 in Table 14. In addition, the maximum unmet energy values are 21,029, 26,817, 20,136, $16,032,20,643$ and 20,934 kWh/yr, for the NE, NW, NC, SE, SS and SW, respectively.

The LOEP and the availability values for the 100\% UD have been presented in Table 10, but the LOEP values for the $150 \%$ UD are $21.99 \%, 28.04 \%, 21.06 \%, 16.76 \%$, $21.59 \%$ and $21.89 \%$, respectively. The corresponding availability values are $78.01 \%$, $71.96 \%, 78.94 \%, 83.24 \%, 78.41 \%$ and $78.11 \%$. The loss of energy hours for the reference demand (i.e. 100\% UD) is 38.5, 110.4, 93.7, 159.4 and 141.9, while the values obtained for the $150 \%$ UD are 1,926, 2,456, 1,845, 1,468, 1,891 and 1,918, respectively. These reliability indices provide important information about how reliable the proposed SPM systems can be in situations when the users' demands are increased.

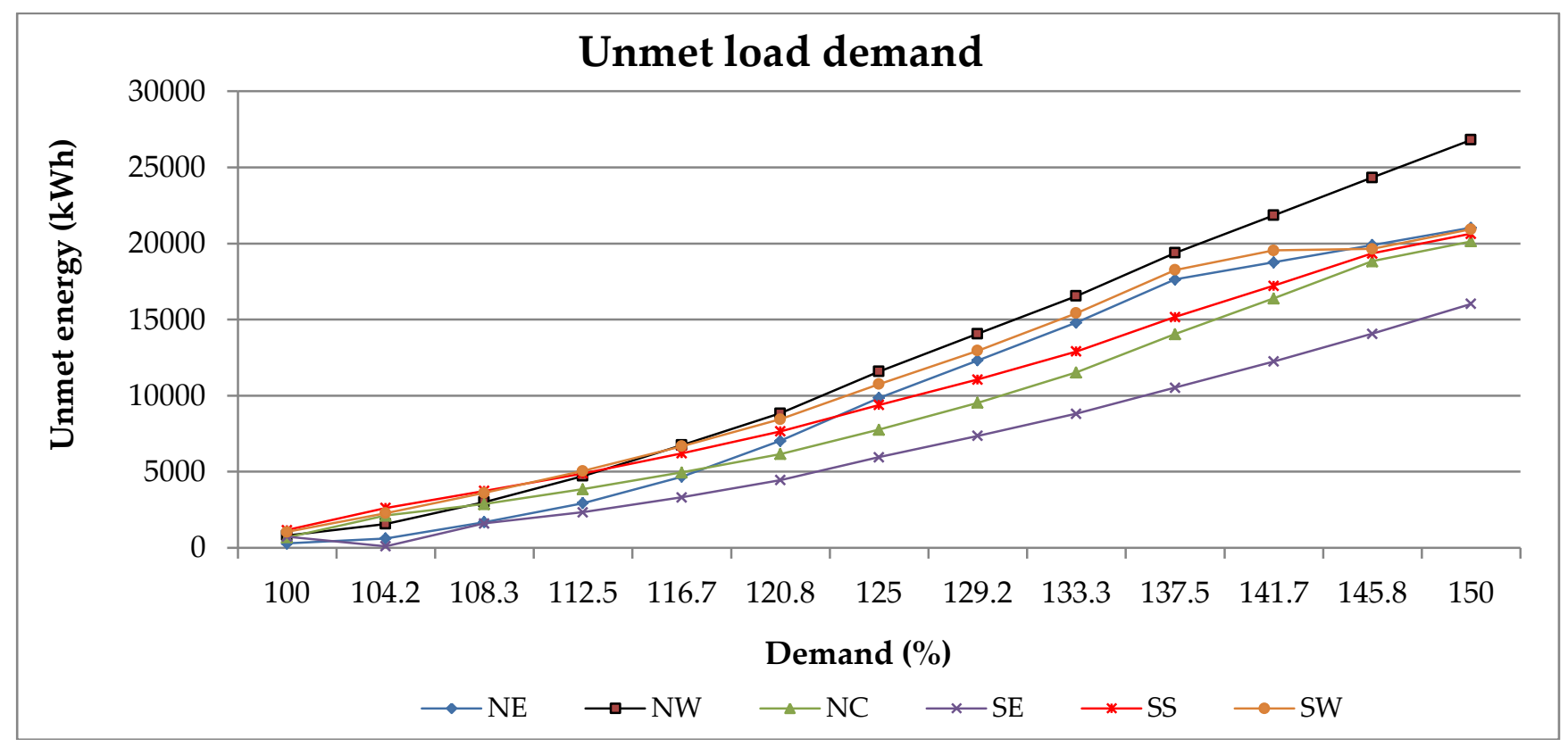

Figure 89: Unmet energy demand for the load growth 


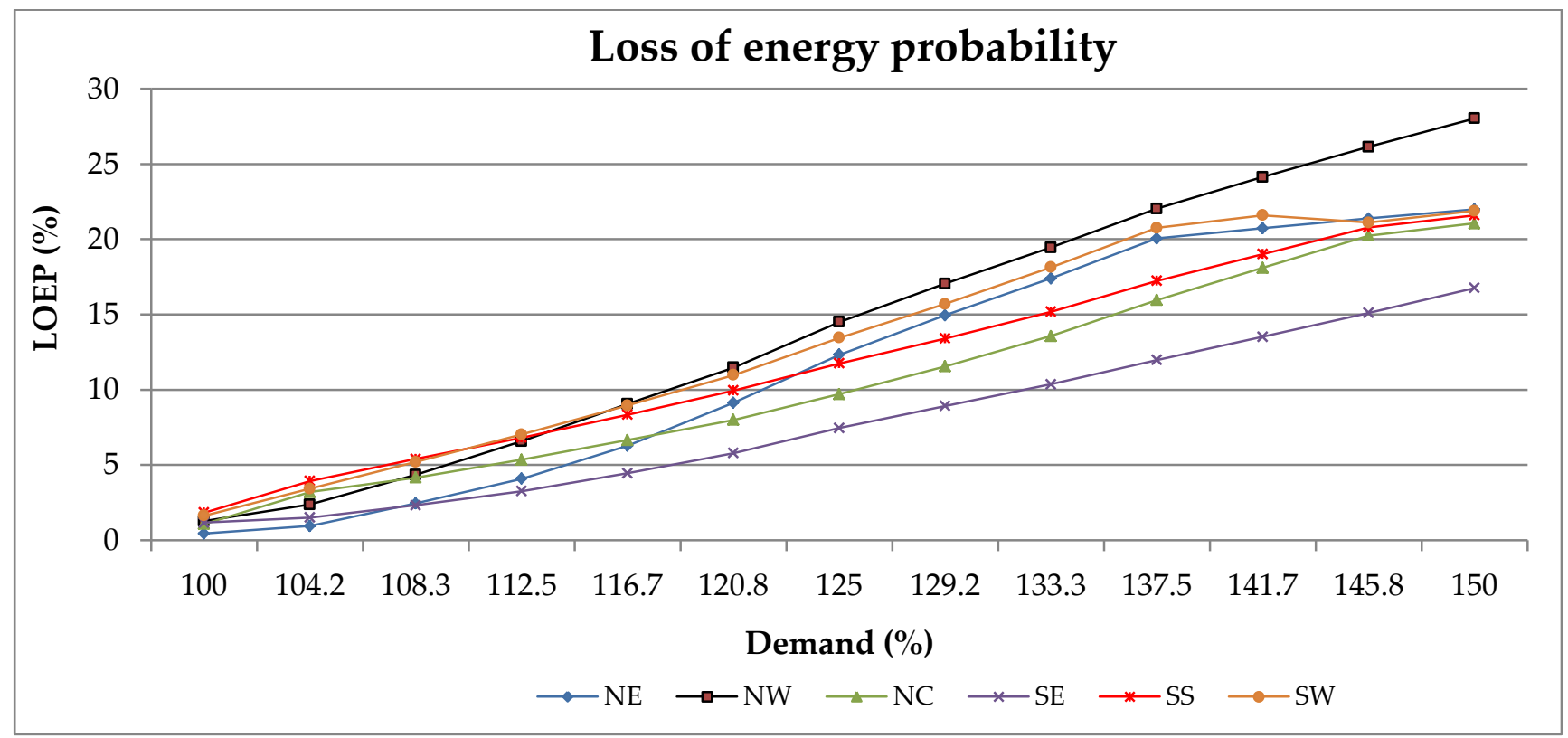

Figure 90: Loss of energy probability for the load growth

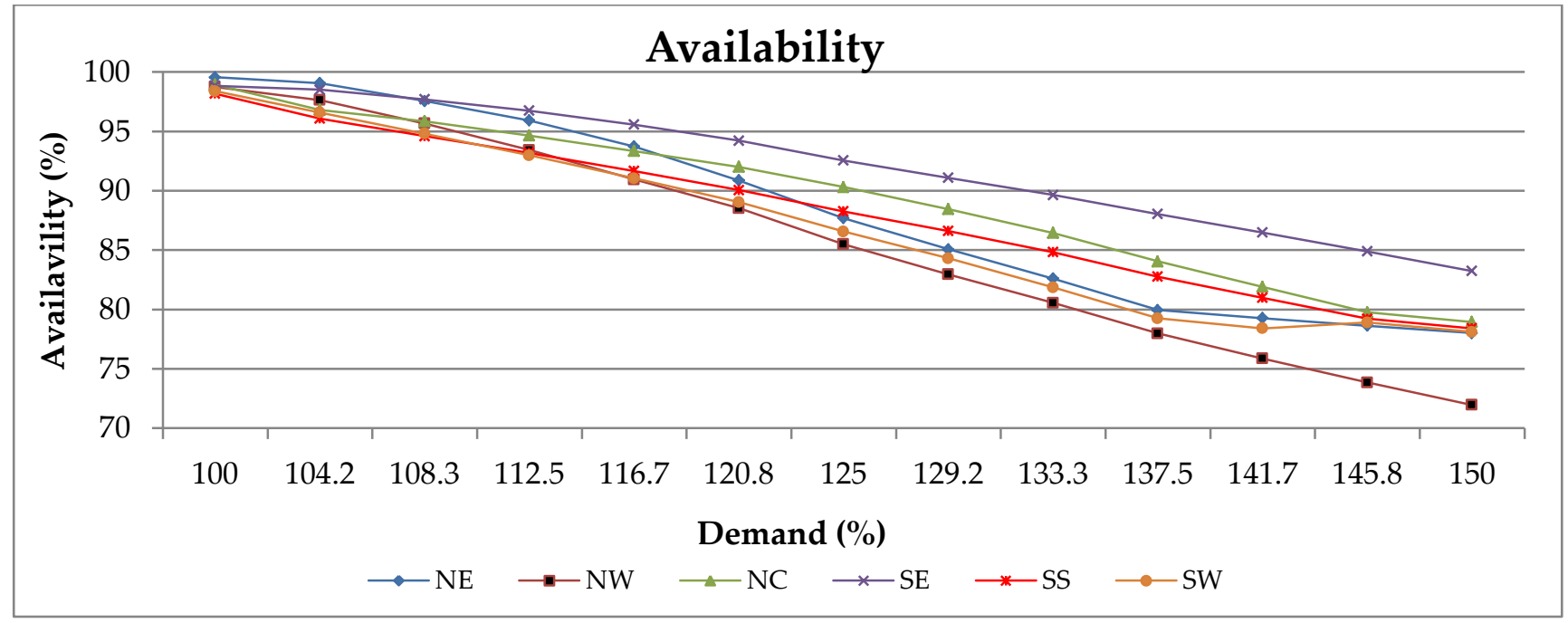

Figure 91: Availability for load growth scenario

\subsection{New SPM capacity with initial battery bank}

\subsubsection{PV capacity}

Based on the foregoing, technical decisions are again required to ensure that the user's future energy demand is accommodated by the proposed microgrid systems. Doing this ensures a reliable energy supply for the intended users. The SPM capacities that can support the new households' load demands are shown in Figure 92. The 
capacities required for the 100 - 150\% UD range from 50 to $94.6 \mathrm{~kW}$ for all the locations. These have been determined from Eq. (15) and the design safety factors reported in Table 15.

These results indicate that the SPM capacities have increased in accordance with the increase in the users' energy requirements.

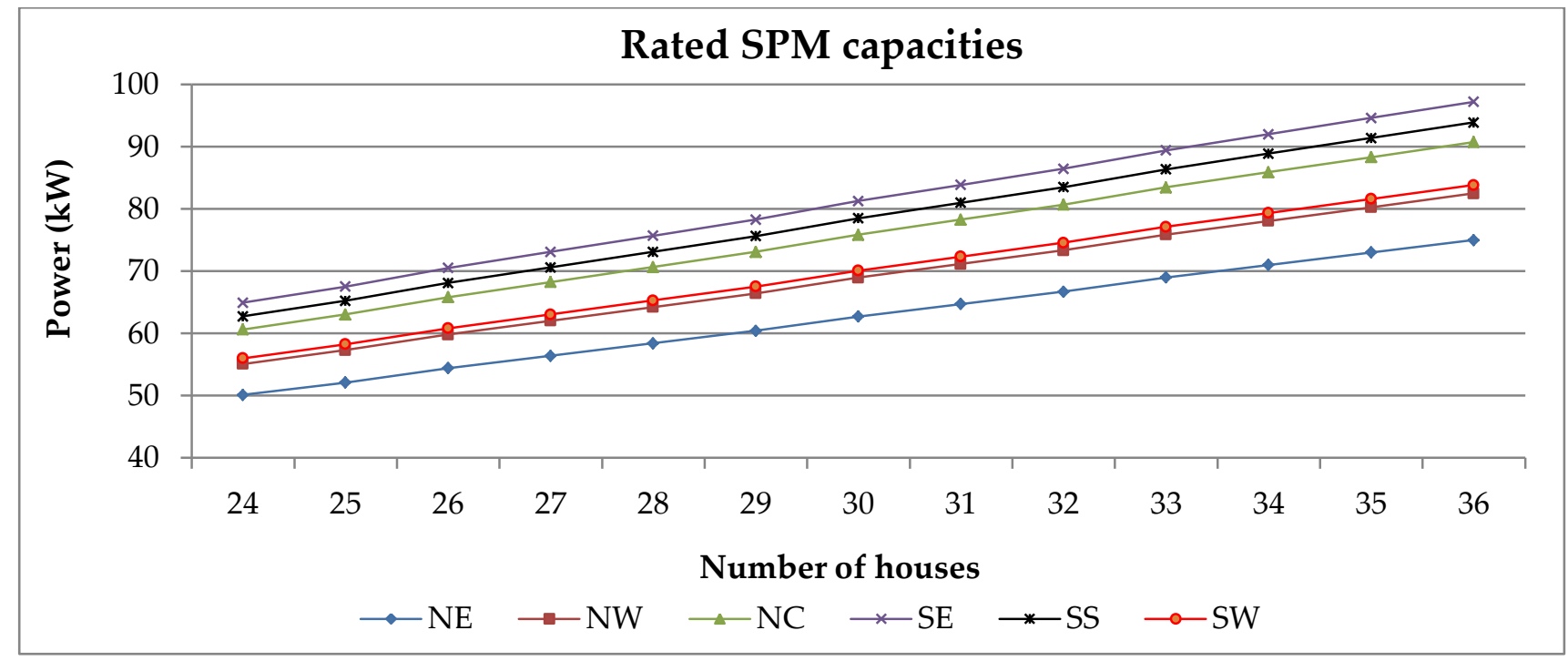

Figure 92: New SPM capacities for the load growth

\subsubsection{Battery SoC and reliability analyses with new PV capacity}

The SoC values obtained for the new SPM system capacities for the $112.5 \%$ UD, 125\% UD, 137.5\% UD and the 150\% UD are shown in Figures 93 to 96. The values for these systems are more than those obtained in Figures 83 to 88. Because the previous results show that the battery SoC decreases as load grows, analysing the battery energy profile in a step of $12.5 \%$ load demand growth is expected to provide a reasonable trend. It is clearly seen that the battery SoC profile has been improved by the new system capacities compared to the initial case shown in Figures 83 to 88.

Figure 97 shows the comparison of the unmet load for the new case with the initial case. The lowest and the highest unmet energy of 2,339 kWh/yr and 26,817 $\mathrm{kWh} / \mathrm{yr}$ are obtained for the initial case for the SE and NW zones (see Figure 89). These values correspond to the $112.5 \%$ UD and $150 \%$ UD, respectively. Similarly, the lowest and the highest unmet energy of $492 \mathrm{kWh} / \mathrm{yr}$ and 5,341 kWh/yr are obtained for the new case for the NE and SW, respectively. These also correspond to the $112.5 \%$ UD and 
$150 \%$ UD. It is obvious from these results that the new case has a lower maximum unmet load demand compared to the initial case. While UDi represents the results for the initial case, UDn stands for the results for the new SPM capacities.

The SPM's $L O E P$ and availability are presented in Figures 98 and 99. The results reveal that that better loss of energy and the availability indices are obtained for the new case compared to the initial case. The LOEP values for the initial case range from 6.81 to $28.04 \%$, for 112.5 - $150 \%$ UD. The values for the new case range from 0.68 to $4.85 \%$.

The LOEP values are reduced, while the availability values are increased in the new case, as shown in Figure 99. The system's availability ranges from 95.15 to $99.32 \%$ for the zones, representing the lowest and the highest values. This expresses the confidence level at which the load demand of those communities can be met over the dry and the rainy seasons, by using increased SPM capacities and a constant battery size.

In line with this thesis's focus - on looking practically at energy systems design and analyses, the issue of load growth for reliability purposes in the future has been carefully examined. While the SPM capacities based on scenarios 1 and 2 presented in Table 11 could be applied to systems with no load growth considerations, the SPM capacities in Figure 92 take into account future increase in users' load demands. This involves new SPM sizes and the same rated battery capacity of $540 \mathrm{kWh}$. Again, it is proven through the results presented in Figures 100 and 101 that the SPM's energy production and the total losses are higher than the values obtained for the scenarios 1 and 2 (see Figure 38 and Table 9). However, the SPM's normalised yields and losses are the same with the values obtained for the initial scenario.

As earlier discussed, the temperature losses are calculated by considering the cell temperature and the temperature coefficient of power, while the other losses are accounted for by using a derating factor of 0.85 . Similar values of the derating factor have been reported in the literature; 0.9 in [205-208]; 0.8 in [209] and 0.75 in [210]. While the UD (ep) represents the energy production, the UD (tl) stands for the total losses with respect to the users' demands. The microgrid system supplying the $112.5 \%$ UD in the NE zone has the highest energy production of $354 \mathrm{kWh} / \mathrm{d}$, while the one supplying 
the $150 \%$ UD in the SS zone has the least energy production of $330 \mathrm{kWh} / \mathrm{d}$. In the same vein, the highest and the lowest energy losses for these two zones are $103 \mathrm{kWh} / \mathrm{d}$ and 59 $\mathrm{kWh} / \mathrm{d}$, respectively.

Furthermore, as earlier mentioned, the SPM system capacities discussed under different scenarios have the same yields and losses. The yields for the new system capacities are 4.72, 4.56, 3.99, 3.84, 3.26 and $4.08 \mathrm{hr} / \mathrm{d}$ for the NE, NW, NC, SE, SS and SW zones, respectively. The corresponding users' demands range from 100 to $150 \%$ UD. Similarly, the losses are 1.37, 1.37, 1.4, 1.05, 0.84 and $1.14 \mathrm{hr} / \mathrm{d}$, respectively. The highest and the lowest values are obtained for the NE and the SS zones (see Figures 39 and 40). These values represent the highest yields and losses for any solar PV system in the considered locations, based on the criteria used in this study. Following these results and analyses, it can be generally concluded that the SPM's performance largely depends on locations.

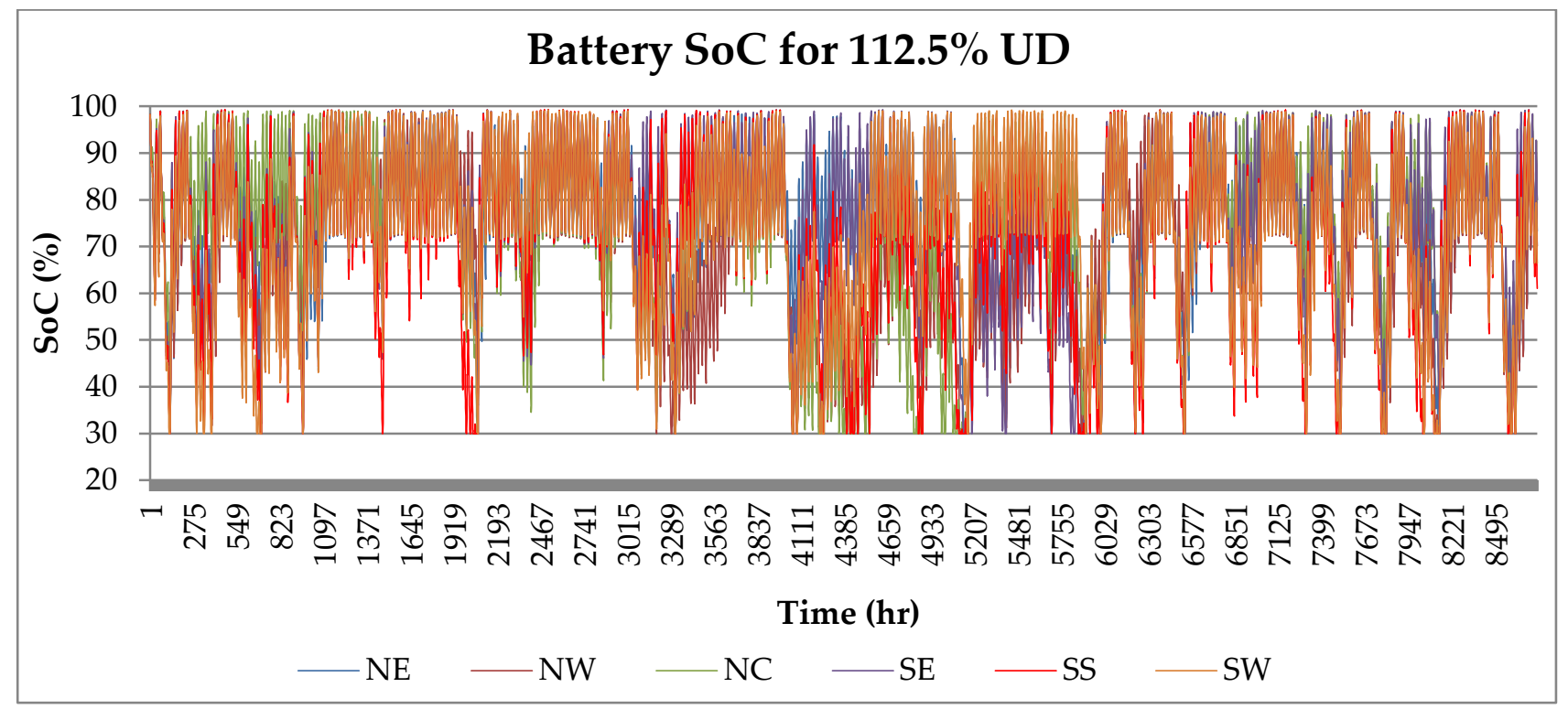

Figure 93: Battery SoC for 12.5\% load growth with new SPM capacities 


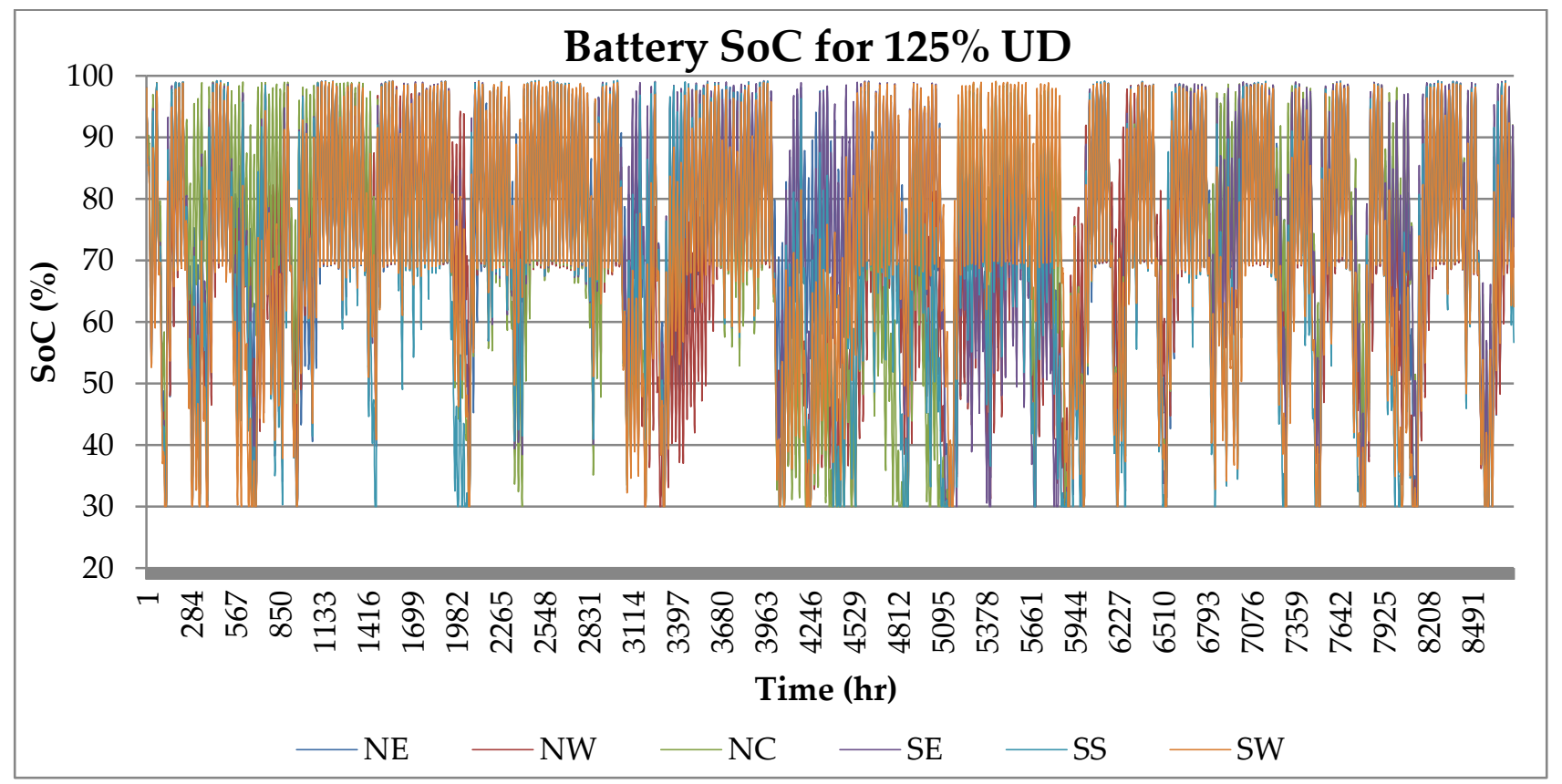

Figure 94: Battery SoC for 25\% load growth with new SPM capacities

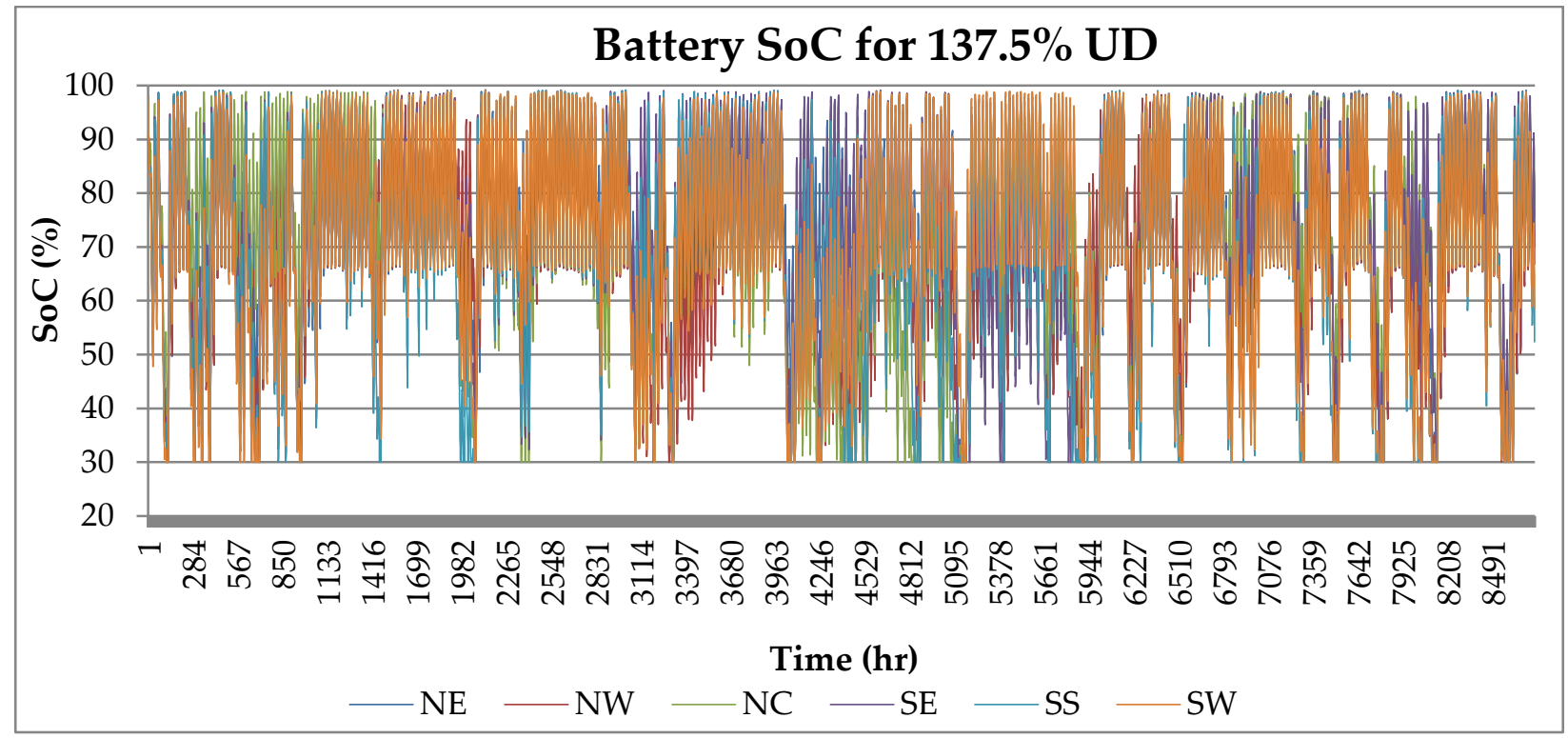

Figure 95: Battery SoC for 37.5\% load growth with new SPM capacities 


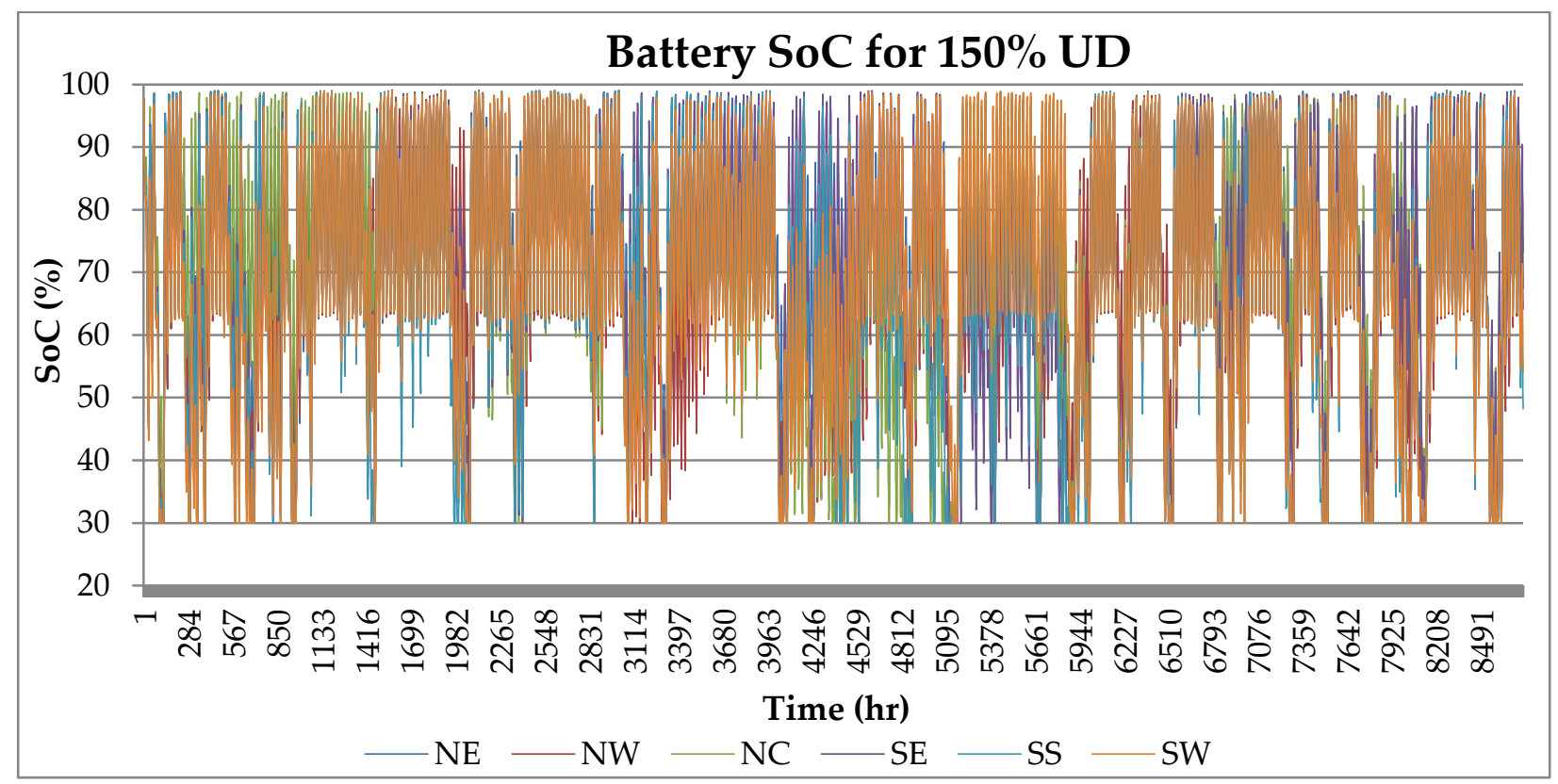

Figure 96: Battery SoC for 50\% load growth with new SPM capacities

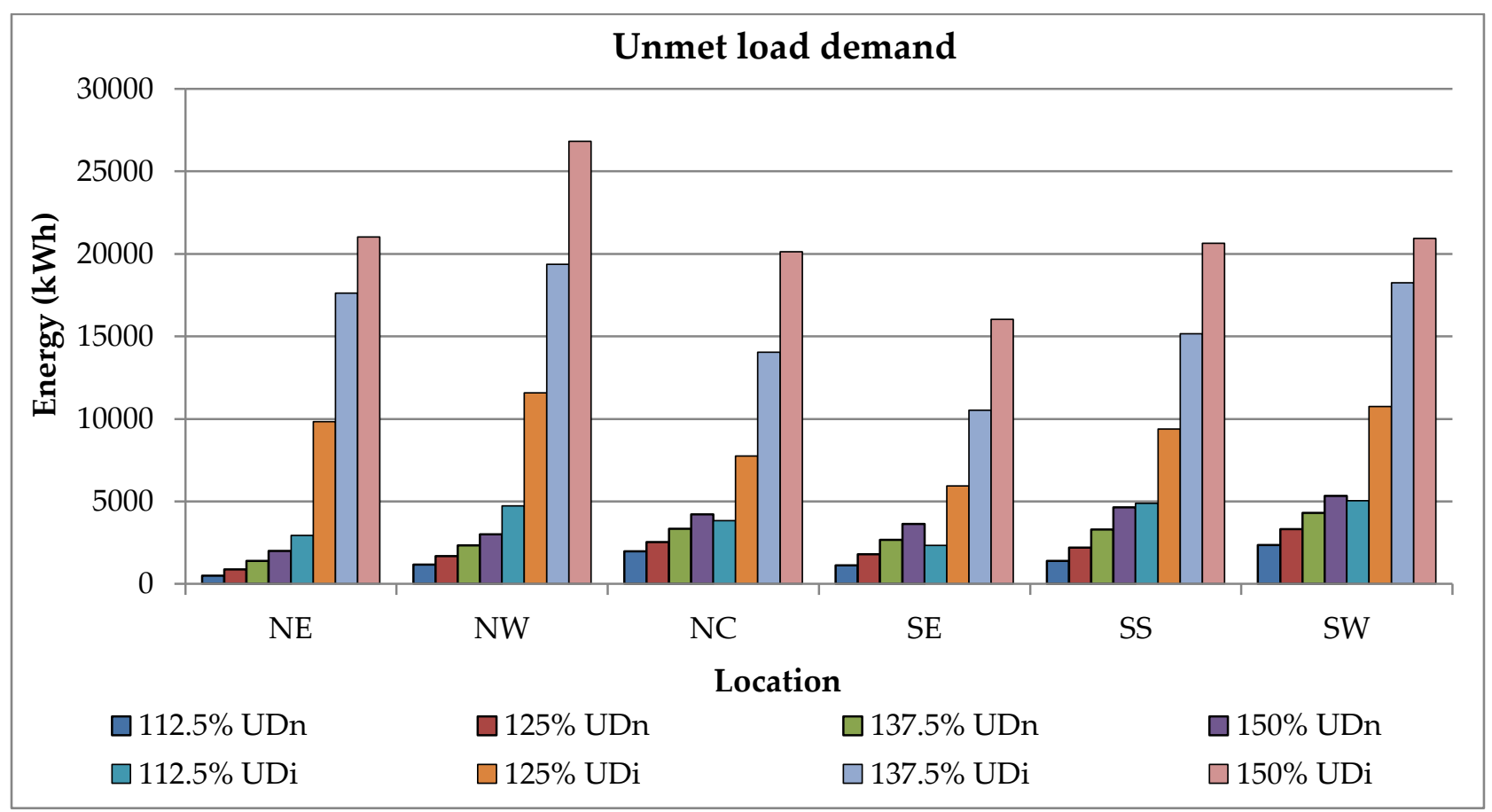

Figure 97: Unmet demand with the initial and the new SPM capacities 


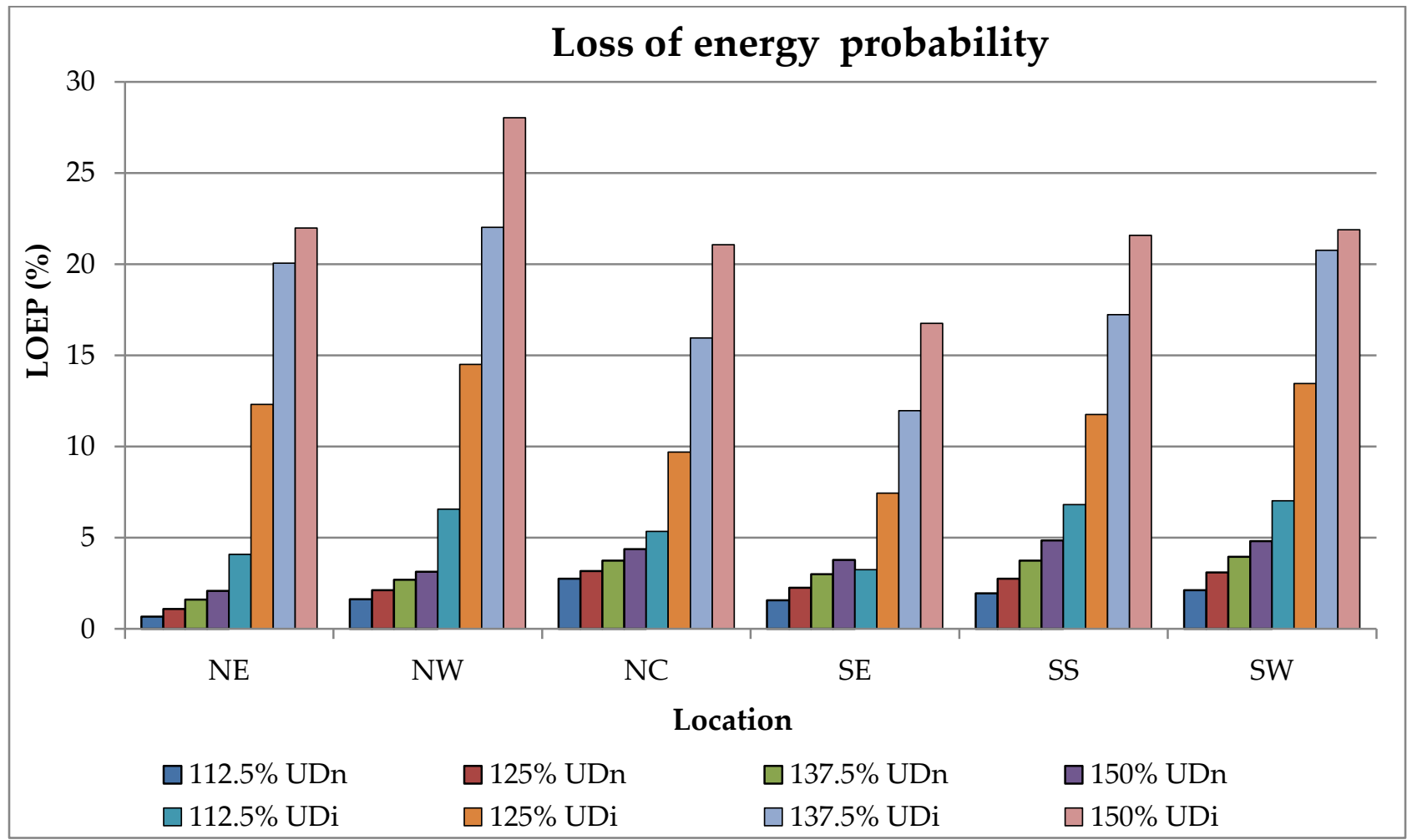

Figure 98: Loss of energy probability with the initial and the new SPM capacities

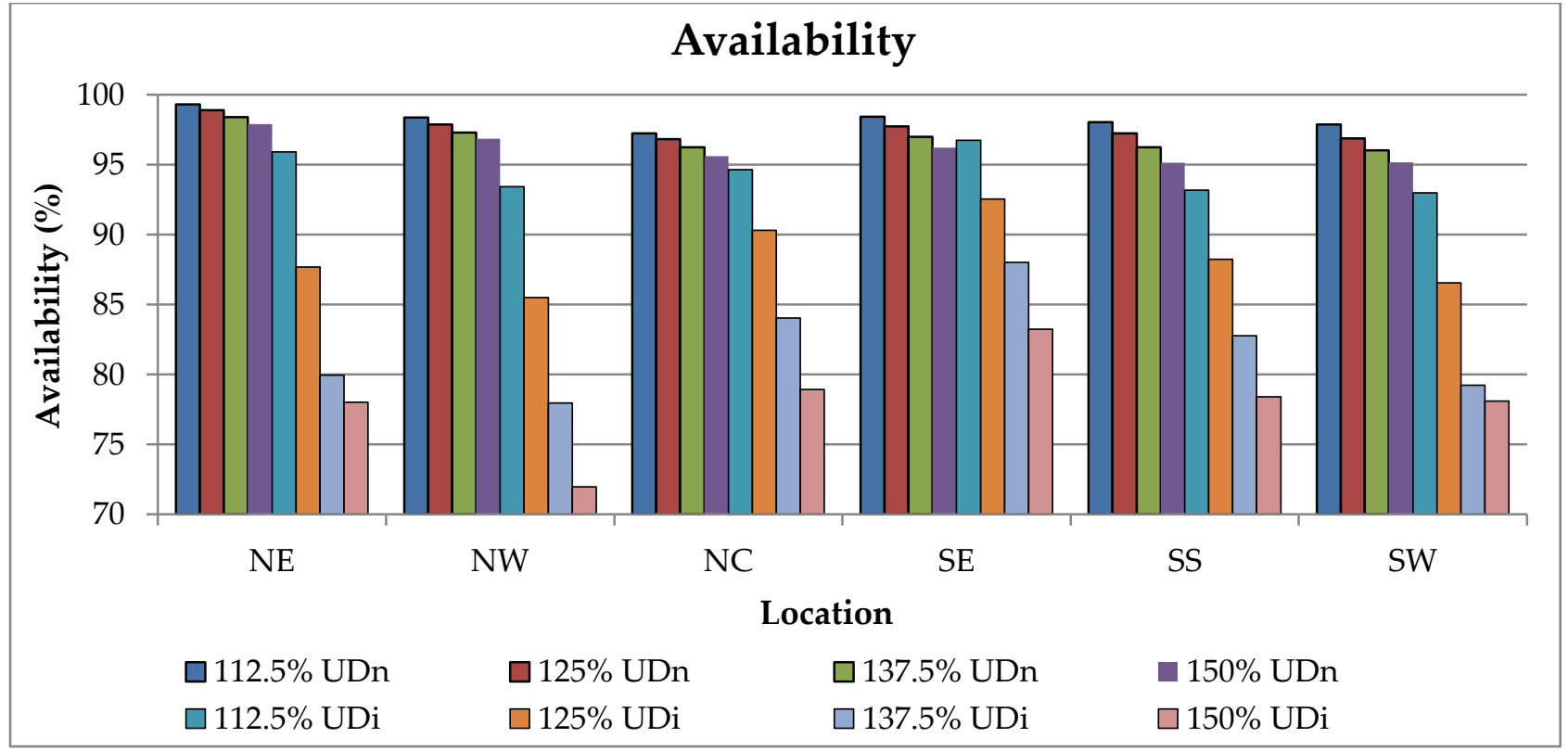

Figure 99: Availability for the initial and the new SPM capacities 


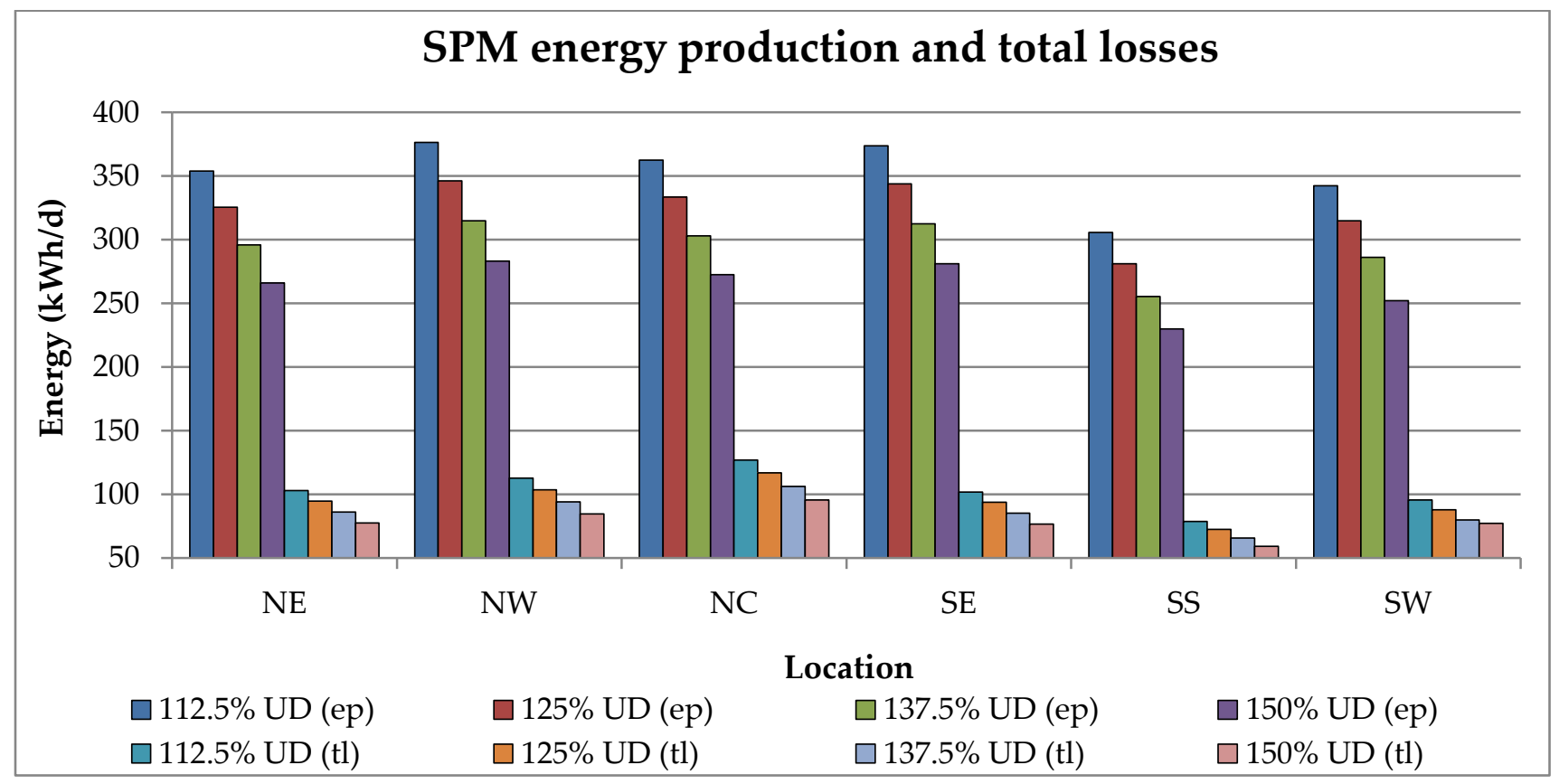

Figure 100: Energy production and total losses for the new SPM capacities

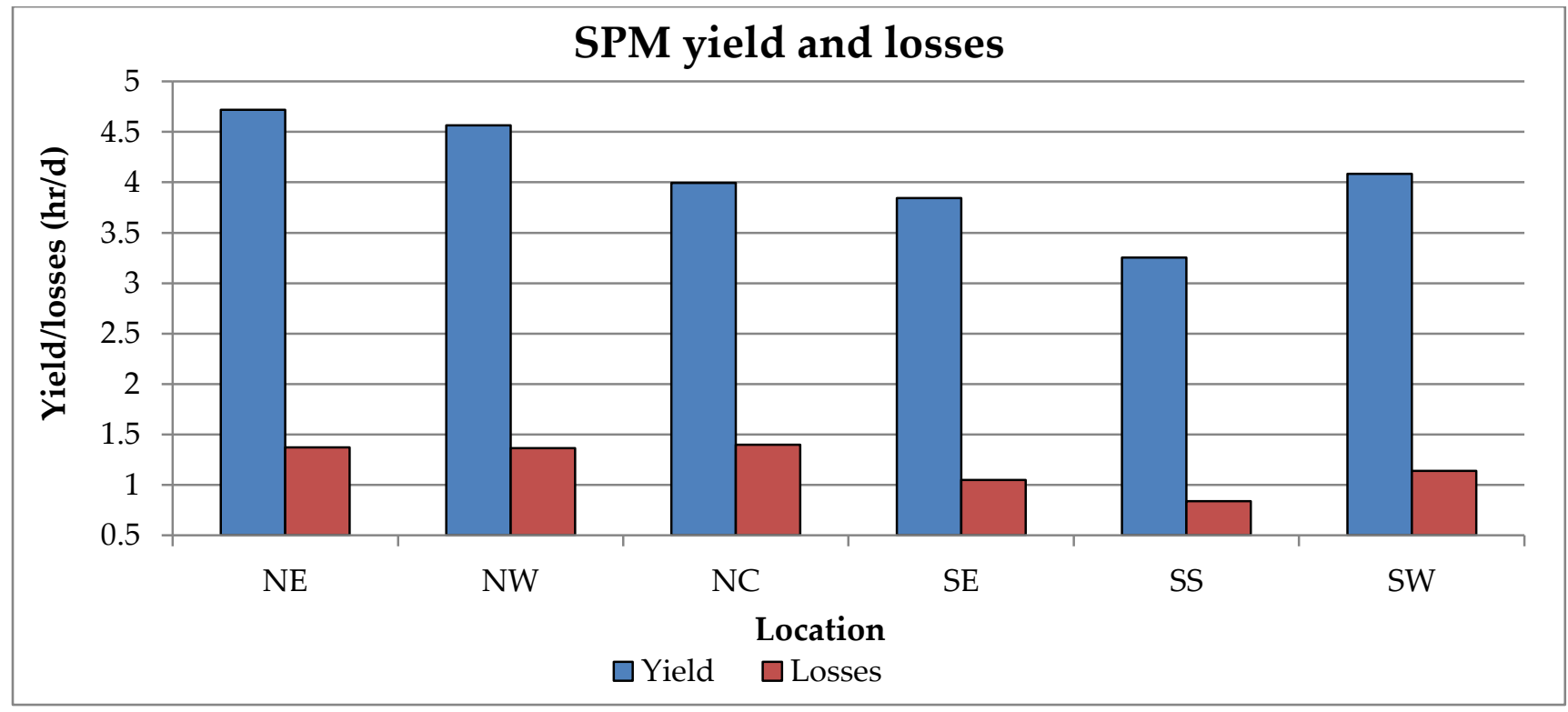

Figure 101: Yield and losses of the new SPM capacities

\subsection{SPM capacity with new battery bank}

The $540 \mathrm{kWh}$ battery bank is arranged in 2 strings of 174 units of $2 \mathrm{~V} 800$ Ah cell to achieve the ampere-hour rating of 1,600 at $348 \mathrm{~V}$ system voltage. From the practical point of view, it is unrealistic to increase battery strings all the time, unless a significant 
increase in the users' energy consumption creates a strong case for the expansion. It should also be noted that the configuration of multiple battery bank's strings in parallel usually leads to poor performance and a shortened life. This is because of the unequal charge and discharge rates between the different strings [157]. For this reason, between 2 and 3 battery strings are considered in this study based on best practices.

Though Table 13 has expressed the relationship between the load demand and the percentage users' demand (\% UD), there is no information over the proposed systems' life span about the time when the increase in demand will occur. A careful technical decision is, therefore, necessary to be able to analyse the practicability of the proposed microgrid systems. Figure 99 reveals that the SPM's availability reduces as the percentage users' demand increases. However, the values obtained for the $125 \%$ UD are higher than those obtained for the $137.5 \%$ UD and the 150\% UD for all the zones, though lower than the values for the $112.5 \%$ UD. The values obtained for the $125 \%$ UD scenario are $98.91 \%, 97.88 \%, 96.83 \%, 97.75 \%, 97.25 \%$ and $96.90 \%$, respectively, for the NE, NW, NC, SE, SS and SW zones. Based on this, the initial battery design is, therefore, assumed for the 100 to $125 \%$ UD, while a new battery capacity is considered for the UDs greater than $125 \%$.

Additionally, the information shown in Table 13 suggests that UDs greater than $100 \%$ (i.e. UD $>63,875 \mathrm{kWh} / \mathrm{yr}$ ) will require more electricity supply from the SPM systems than the initial case of $63,875 \mathrm{kWh}$. In this vein, more energy supply is needed to satisfy the load growth. While the new SPM capacities and the initial battery size are considered for up to $25 \%$ load growth (i.e. 125\% UD), the new SPM sizes and the new battery bank can be considered for the load growth of $>25 \%$ to $75 \%$. A load growth of $75 \%$ corresponds to $175 \%$ UD, which is $111,690 \mathrm{kWh} / \mathrm{yr}$. Substituting this information into Eq. (28), with other parameters remaining unchanged, the new battery capacity is $826 \mathrm{kWh}$. This has an ampere-hour rating of 2,374 and the new battery arrangement will, therefore, require 3 strings of 174 units of $2 \mathrm{~V} 800$ Ah cell.

\subsubsection{New PV and battery bank capacities}

The hourly SoC values for the 150\% and 175\% UDs presented in Figures 102 and 103, respectively, show the battery bank's energy profiles in the different locations, using the new PV and battery capacities. The relatively lower solar energy generation during the rainy seasons results in lower SoC values. This is noticeable in all the figures illustrating the battery SoC over the 8760-hour period. The unmet load demands for the 
150\% and 175\% UDs are shown in Figure 104 and are also found to be lower than the initial and new values obtained for the $150 \%$ UD in Figure 97. This stems from the increase in the battery bank's capacity, which is able to accommodate a relatively higher energy discharge.

The system in the NE zone has the lowest unmet loads of 399 and $922 \mathrm{kWh} / \mathrm{yr}$ for the $150 \%$ and $175 \%$ UDs, respectively, while the highest values of 2,889 and 4,911 $\mathrm{kWh} / \mathrm{yr}$ are obtained for the $150 \%$ and $175 \%$ UDs in the SS zone. Also, the loss of energy probability for the systems serving the $150 \%$ and $175 \%$ UDs is shown in Figure 105. This is also found to be lower than the initial and the new values obtained for the $150 \%$ UD in Figure 99. The LOEP of $0.42 \%, 1.21 \%, 2.33 \%, 1.15 \%, 3.01 \%$ and $2.29 \%$ is obtained for the $150 \%$ UD, while $0.83 \%, 1.80 \%, 2.87 \%, 1.80 \%, 4.38 \%$ and $3.60 \%$ is obtained for the $175 \%$, respectively.

The system's availability for the 150\% and 175\% UDs are presented in Figure 106. It is obvious that the values are higher than those shown in Figure 99. The values of $99.58 \%, 98.79 \%, 98.84 \%, 96.99 \%$ and $97.70 \%$ are obtained for the $150 \%$ UD, while the values of $99.17 \%, 98.20 \%, 97.13 \%, 98.20 \%, 95.62 \%$ and $96.40 \%$ are obtained for the $175 \%$ UD, respectively. The highest and the lowest availability are obtained for the systems in the NE and SS zones, respectively. These results again reflect the disparity in the SPM's performances in different locations. In this case, the energy system in the SS zone has the lowest performance indices compared to the other zones (see Table 9, Figures 39, 40, 100 and 101).

It can also be observed that the reliability indices for the new PV and battery capacities are better than those of the $150 \%$ UD with initial battery capacity. This is because of the larger battery bank resulting in a relatively lower depth of discharge, compared to the initial battery bank. Increasing the PV and the battery capacities can achieve higher system reliability. However, it will incur higher costs. 


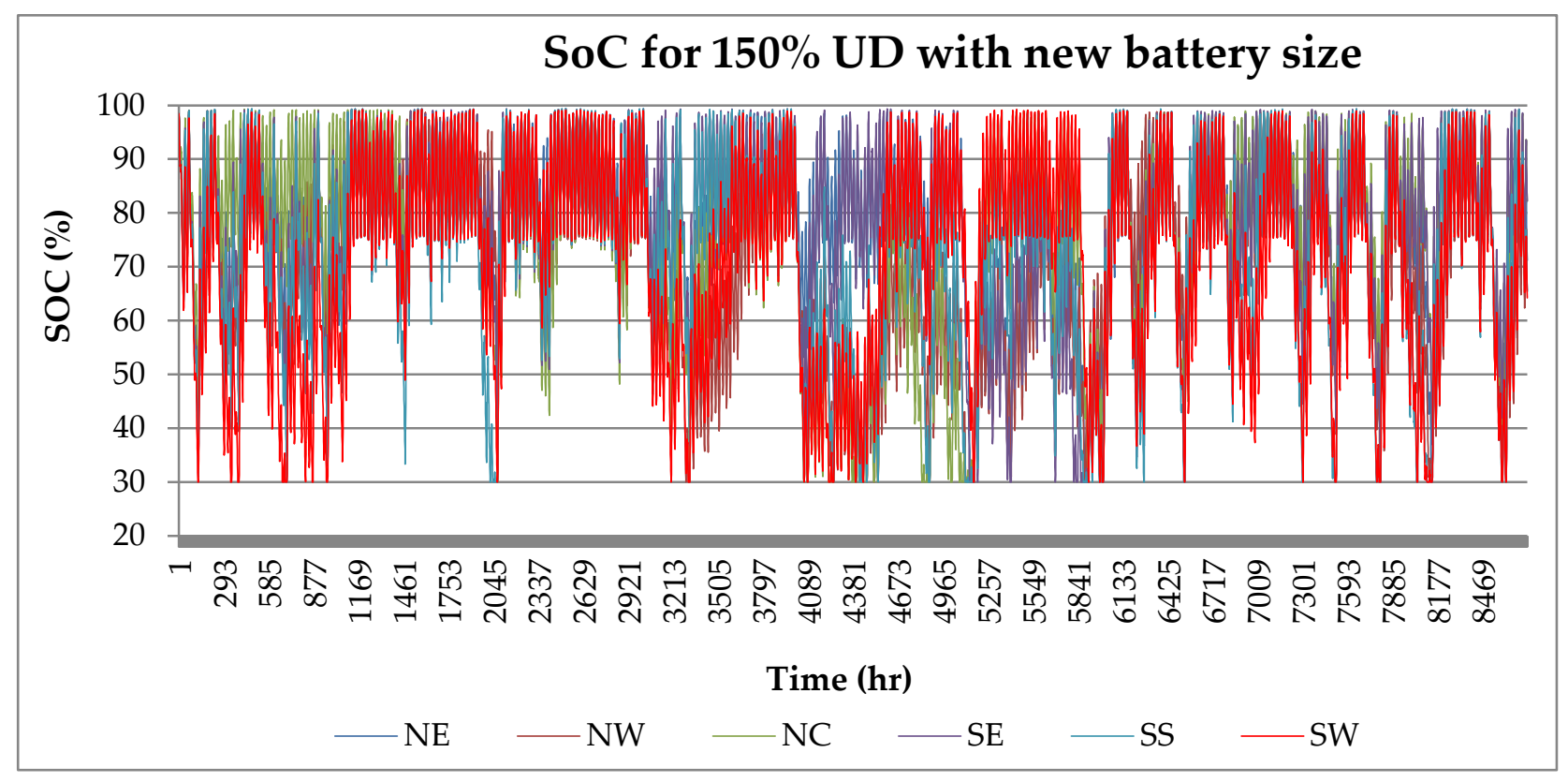

Figure 102: Battery SoC for 150\% UD with new PV and battery capacities

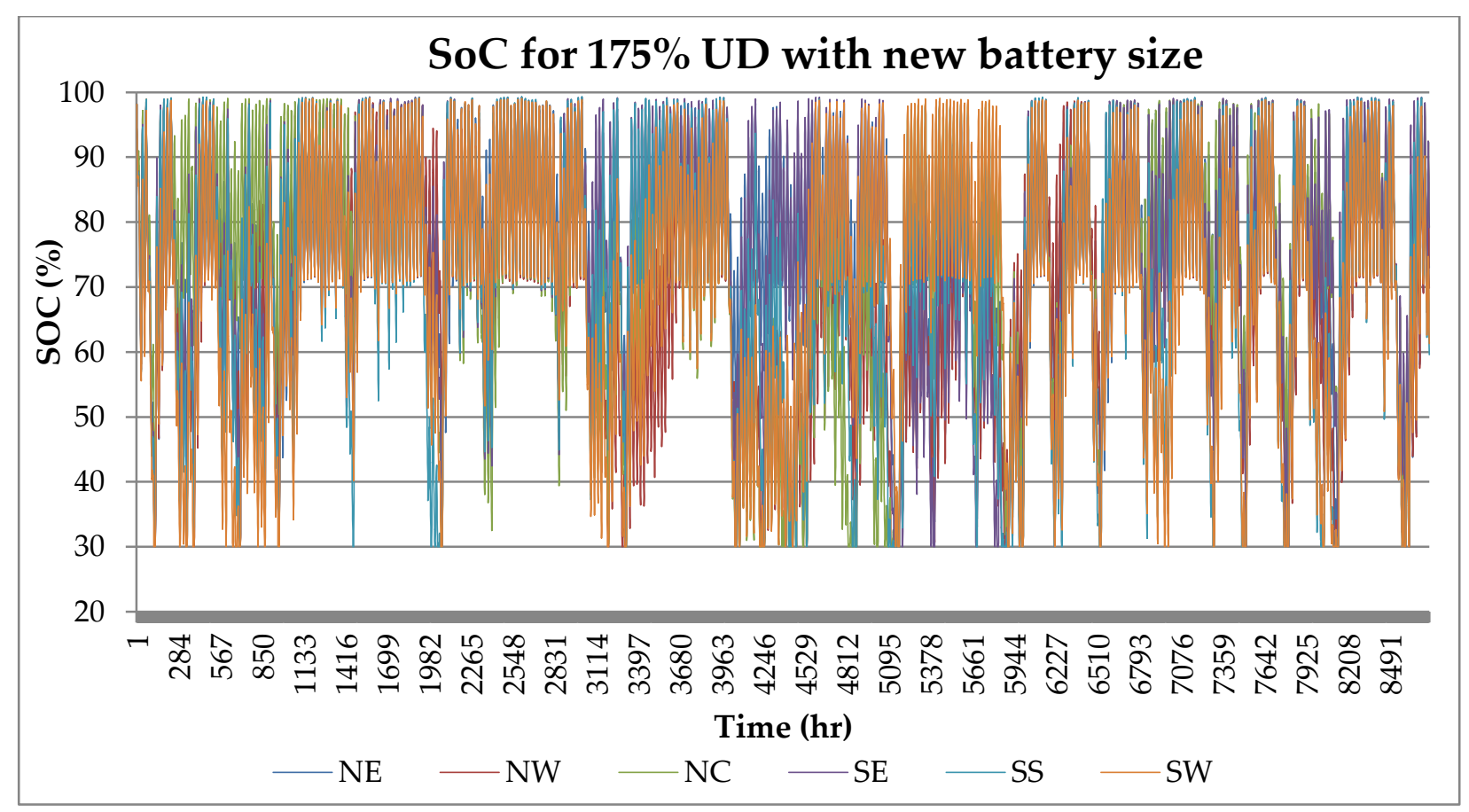

Figure 103: Battery SoC for 175\% UD with new PV and battery capacities 


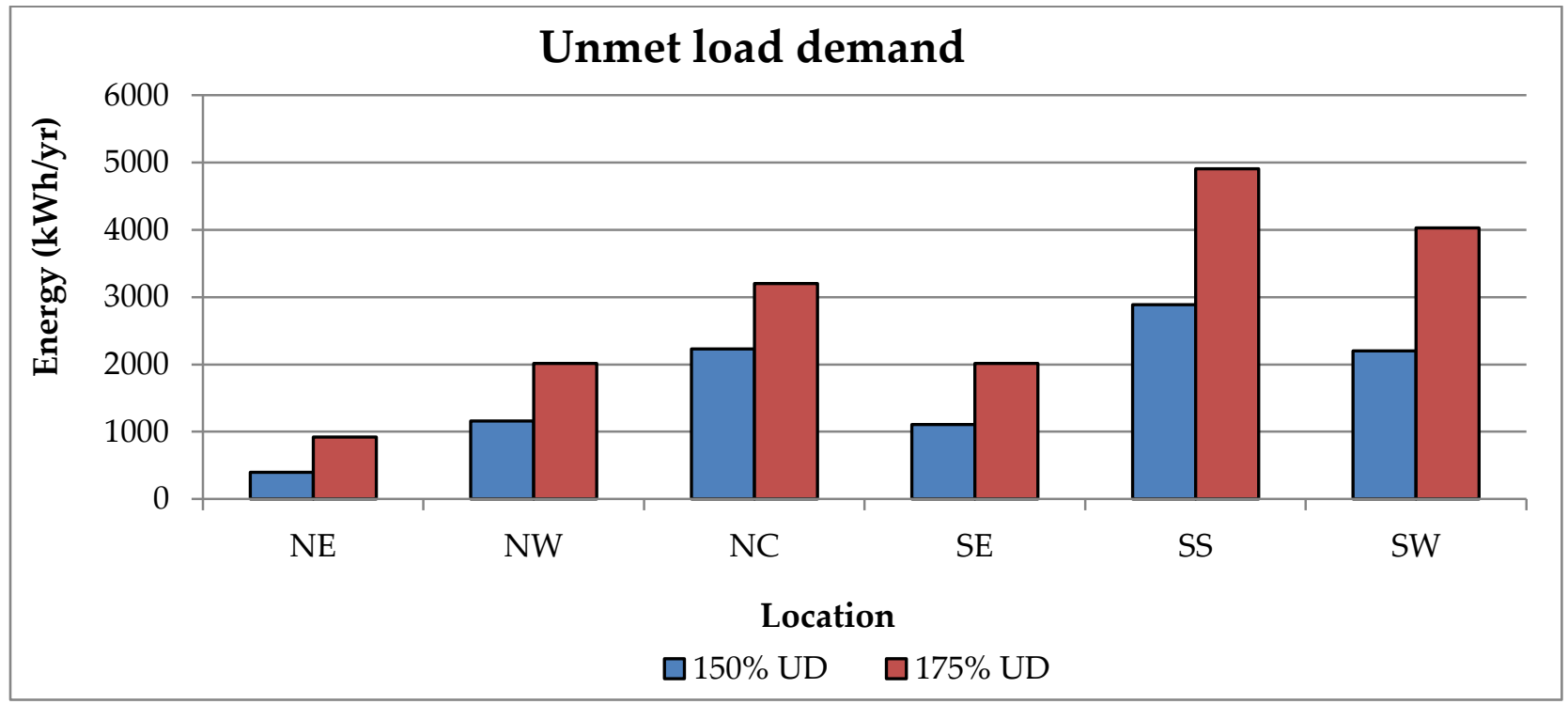

Figure 104: Unmet energy demand for the new PV and battery capacities

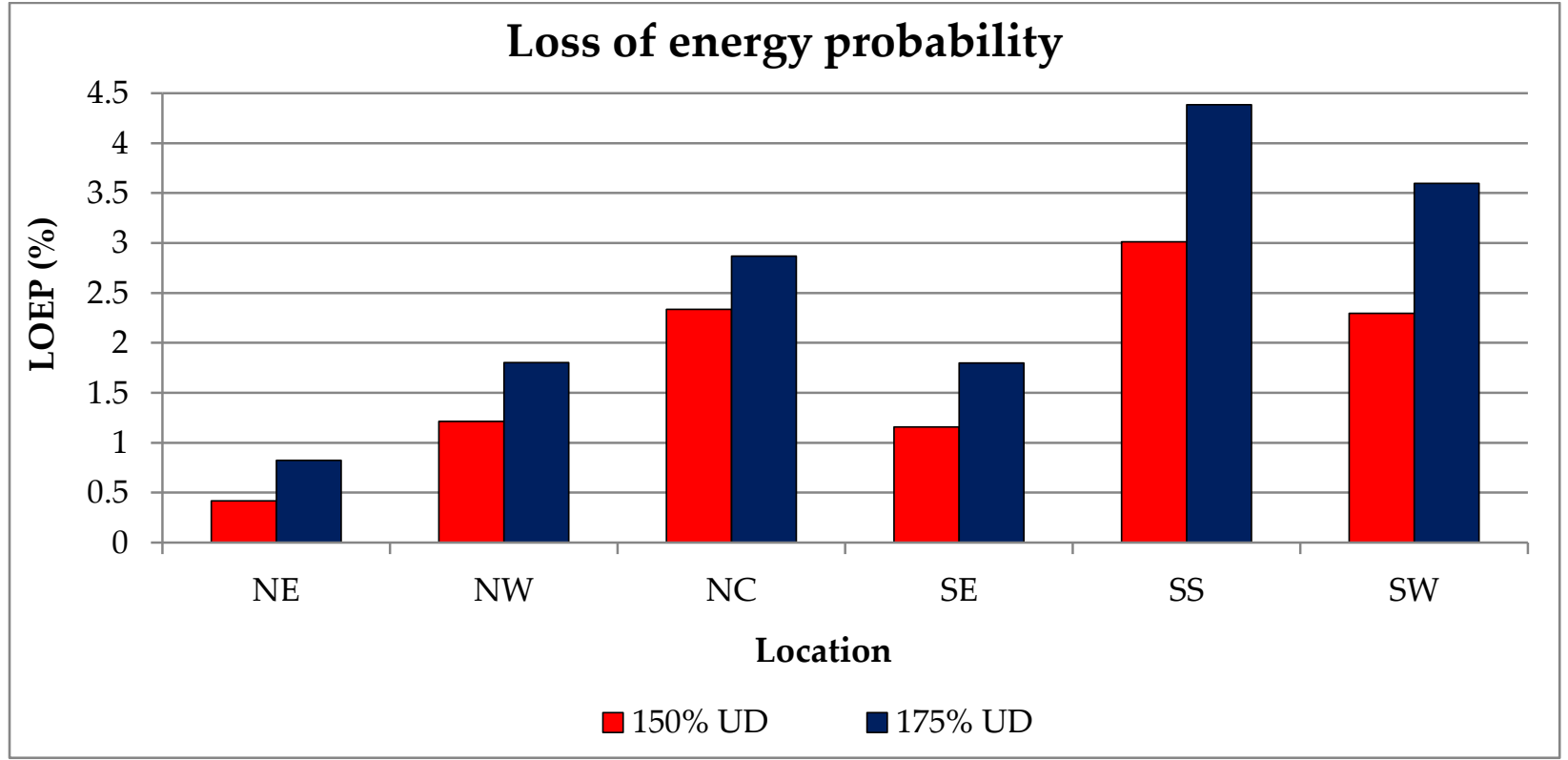

Figure 105: Loss of energy probability for new PV and battery capacities 


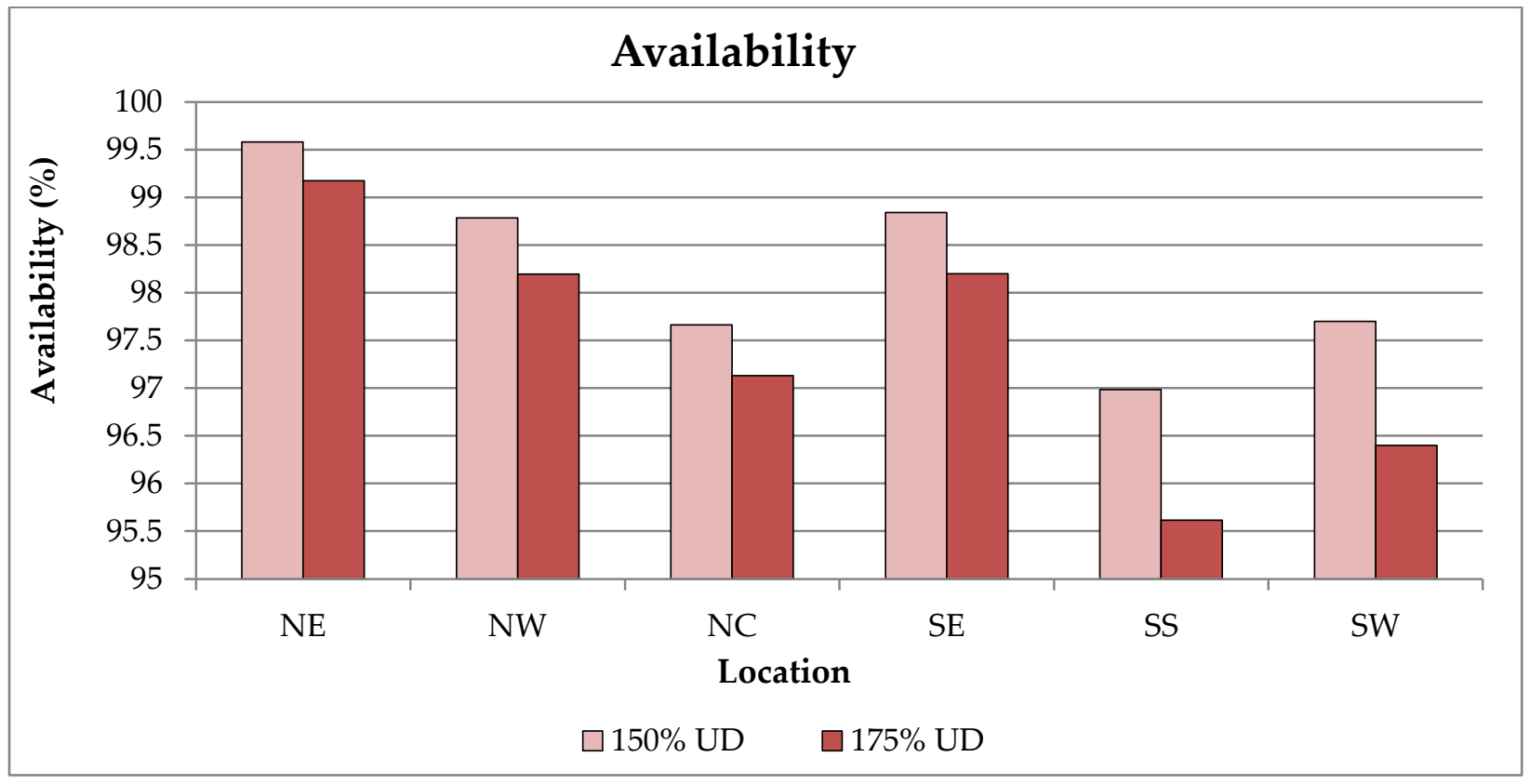

Figure 106: Availability for the new PV and battery capacities

The results have shown that the SPM system's availability depends on the users' energy consumption, the rated capacities and final outputs. The SPM's configuration for the locations differs, while similar battery bank and inverter sizes and the users' load demands have been considered. The maximum number of NASENI PV modules that is required in series is 15 for all the SPM capacities. The series-connected modules produce a total voltage of $668 \mathrm{~V}$. However, the number of strings of the PV modules differs. In the initial scenario, the number of strings is 14, 14, 19, 21, 21 and 16 for the SPMs in the NE, NW, NC, SE, SS and SW zones, respectively. The number of strings for scenario 1 is 18, 19, 21, 23, 22 and 20. The initial and the new battery banks are 540 and $826 \mathrm{kWh}$, respectively. The battery cells assumed in this thesis analysis are manufactured by the Vision Batteries [192]. The ETS $60 \mathrm{kVA}$ inverter/controller [193] has also been sized to support the peak users' load capacities shown in Figures 107 (a) to (e) for the different number of houses.
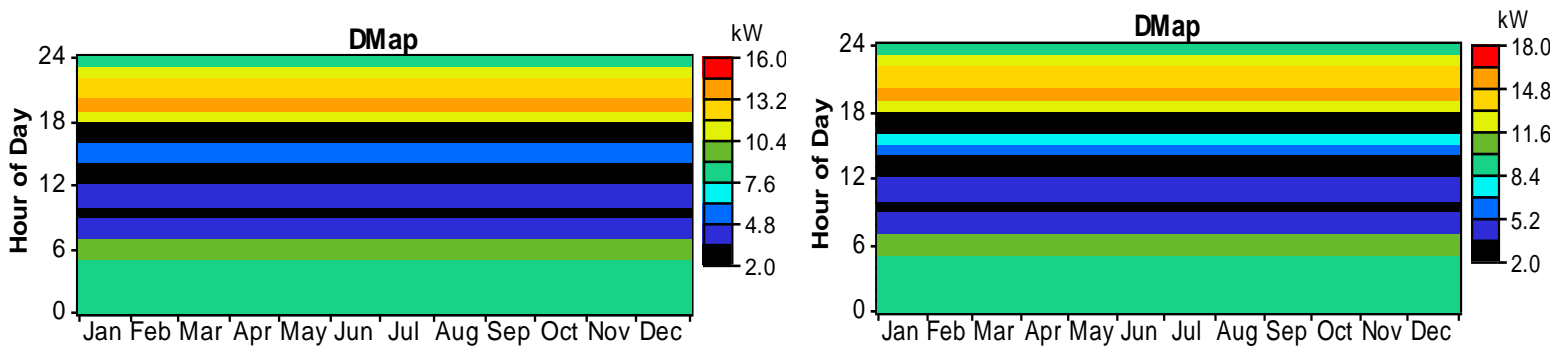
(a)

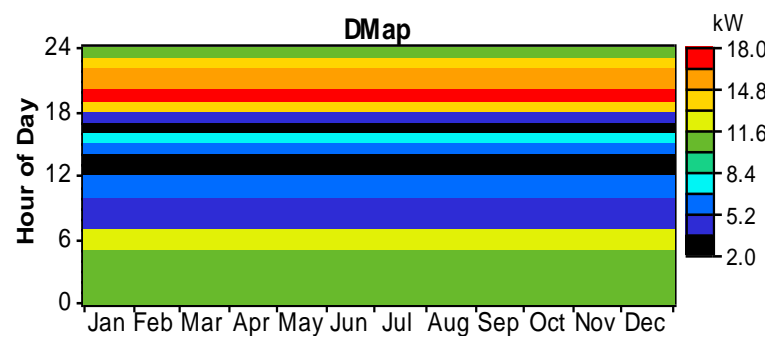

(c) (b)

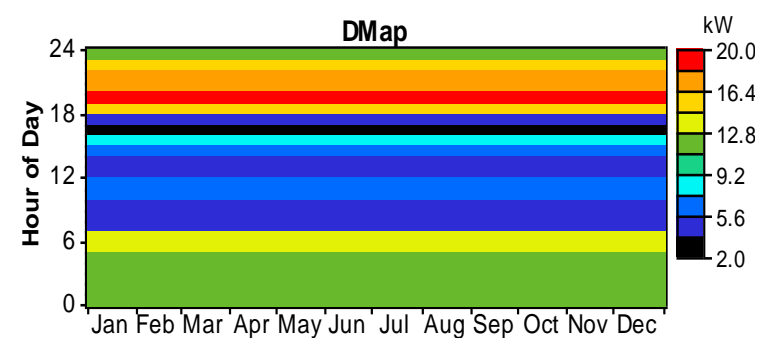

(d)

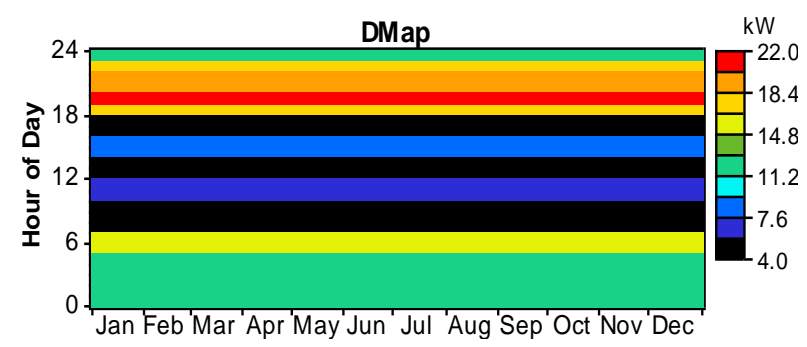

(e)

Figure 107 (a) - (e): Load capacities for the 100, 112.5, 125, 137.5 and 150\% UDs

\subsection{Load growth factor}

The author's field experience has identified that the failure to consider users' load growth over the energy system's life span is one of the major factors responsible for increased PV system failure in several locations in Nigeria. The users' load growth is usually due to one or all of the following factors:

- the change in the users' status

- the increase in the number of users

- the use of inefficient appliances

The thesis, therefore, attempts to address this issue by proposing design approaches and analyses that consider both the present and the future community's electricity demands. The load growth factors $\left(l_{8 f}\right)$ for the analyses earlier discussed range from 1.042 to 1.500 and are used to estimate the SPM's sizes required to support the users' load demand growth. While the $d_{s f}$ quantifies the system's capacities that will compensate for the energy losses, the $l_{g f}$ estimates the system's ratings that can support the users' increased energy consumption. Practically, these two factors are the correction factors that are used to determine the suitable SPM capacities for the specified communities. 


\subsection{Chapter summary}

The chapter has presented the scenarios, detailed results and analyses of the proposed solar photovoltaic microgrid systems with load demand growth. These involved evaluating the impact of the users' load growth on the existing microgrid capacities, in terms of the battery state of charge, unmet load, loss of energy probability and availability parameters. This has provided useful information about the extent to which the available microgrid systems can withstand the increase in the users' load demand requirements. Such information is vital for decision-making and planning.

The chapter has also presented new SPM capacities with the initial battery size, which have relatively higher reliability indices. The techno-economic design for these systems is also compared with the scenario of new PV and battery capacities to find out the effect of increasing the energy storage capacity. The results have shown that the SPM's availability depends on the users' energy consumption, the system capacities and the final outputs. The SPM's configuration for the locations differs, while similar battery bank and inverter sizes and the users' loads have been considered.

The thesis has carefully presented the analyses that include both the losses and the users' increased demand. This can deepen the understanding and planning of offgrid energy systems.

The results presented in the chapter also prove that the SPM's energy production and total losses, expressed in $\mathrm{kWh}$, increase with capacities. However, the system's normalised yields and losses, expressed in $\mathrm{kWh} / \mathrm{kW}$ or $\mathrm{hr} / \mathrm{d}$, are the same for different sizes. This implies that SPM systems in the same location have the same yields and losses. While the temperature losses have been assessed by considering the array temperature and the temperature coefficient of power, the other losses - incomplete utilisation of solar irradiation and the BOS inefficiencies of the BOS, are accounted for by the derating factor.

The chapter also demonstrates that the battery SoC values decreases as the load increases. In addition, the initial SPM system's availability reduces to as low as $\sim 78 \%$ due to the impact of users' load growth. The implication of this is that the users will experience a loss of energy for $\sim 22 \%$ of the time during the year. However, by increasing the microgrid capacities in proportion to the users' increased load requirements, the availability ranging from $95.15 \%$ to $99.32 \%$ can be obtained. This 
expresses the confidence level at which the communities' load demand may be met over the dry and the rainy seasons. The cost implications of the different microgrid capacities are discussed in the next chapter. 


\section{Chapter 7}

\section{Economic performance of the Microgrid}

This chapter discusses the proposed microgrid system's economic performance, both with and without the users' load demand growth. It presents a detailed life cycle cost assessment of the different system capacities that is then compared with that of a diesel power plant. The chapter is divided into three main parts. The first presents the cost implications of the microgrid systems with no users' load demand growth. This is considered in terms of the initial capital cost, percentage cost of the PV and the battery components, operation and maintenance cost, life cycle cost (LCC) and the cost of energy (COE). The second part discusses the energy system's economic analysis with the user's load growth, while the third section compares the proposed solar photovoltaic microgrid's costs with that of a diesel power plant. A summary concludes the chapter.

\subsection{Cost of systems with no load growth}

\subsection{Initial capital cost}

The photovoltaic system's initial capital costs for the different zones are shown in Figure 108. The cost analysis with no load growth implies that the initial scenario and the scenario 1 are based on microgrid capacities for the 100\% users' demand (UD). The initial costs for the initial scenario are $\$ 144,498$, \$144,507, \$159,290, \$166,336, \$166,346 and $\$ 151,433$ for the NE, NW, NC, SE, SS and SW zones, respectively. Similarly, the values obtained for the scenario 1 are $\$ 154,912$, \$ 160,183, \$165,432, \$170,676, \$168,585 and $\$ 161,235$. These results demonstrate that the initial costs are proportional to the system's capacities, with those in the NE and NW zones having the lowest costs for the two scenarios, compared to the systems in the other zones. 


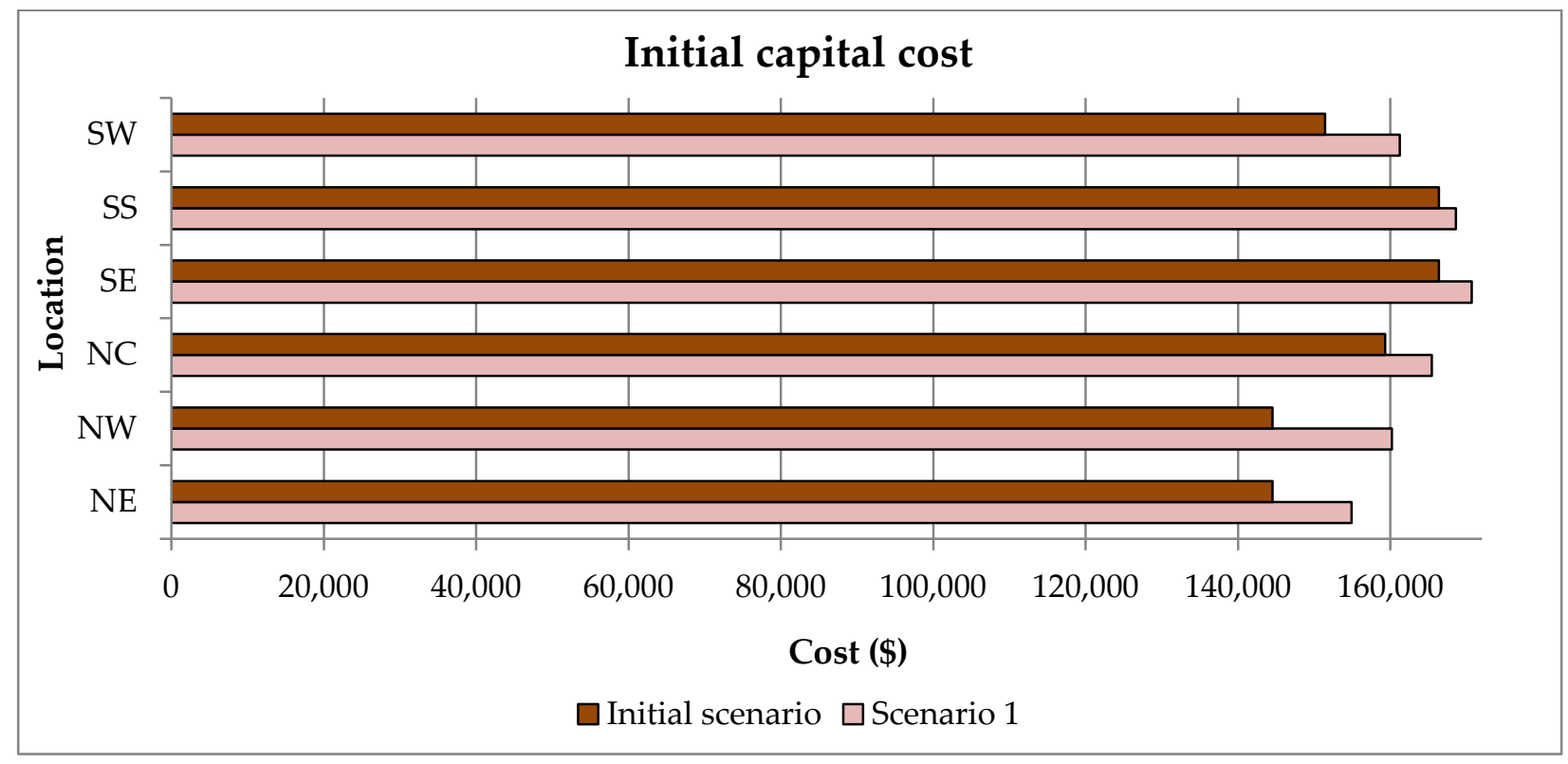

Figure 108: Initial capital costs of the microgrid systems with no load growth

\section{1. 2 Percentage costs of PV and battery components}

The initial capital investment involves the cost of the solar photovoltaic array and the balance of system - battery bank, inverter/controller, capacitor, cables etc. It also includes the installation cost. The percentage costs of the photovoltaic array and the battery systems are shown in Figure 109.

Furthermore, the battery bank has relatively higher initial costs for most of the data sets. The solar photovoltaic arrays' costs for the initial scenario from 32 to $40.5 \%$ of the initial cost, while the battery bank's cost ranges from $\sim 40$ to $46 \%$. Similarly, the photovoltaic arrays' costs range from $\sim 36$ to $42 \%$ and the battery cost ranges from $\sim 40$ to $43 \%$ of the initial cost for the scenario 1 . This clearly shows that the PV modules' and the battery bank's costs constitute a significant portion of the SPM's initial capital costs.

From a practical point of view, achieving economically feasible PV-based energy solution is dependent on how well the PV and the battery components are designed. For instance, a too-large battery bank design will invariably lead to a high initial capital cost. This is because while the PV module costs are currently being reduced, the battery storage systems are still expensive. In this thesis, the battery system has been designed to support the worst-case users' energy consumption with a $20 \%$ capacity for contingencies. The solar PV modules are modeled to provide the required charging 
current for the battery bank. The technical analysis allows the PV array capacities in proportion with the users' load growth while using the same battery bank. The battery bank is configured in 2 strings of 174 series-connected 2V 800 Ah cells. Such a design provides cost effective solutions compared to increasing the battery system to 3 strings of 174 series-connected 2V 800 Ah cells. Considering the huge financial investments that will be committed to the PV/battery components, it is necessary that they are carefully designed. This study has, therefore, undertaken an in-depth PV/battery component analysis to achieve the techno-economic results that are comparable to those in the literature.

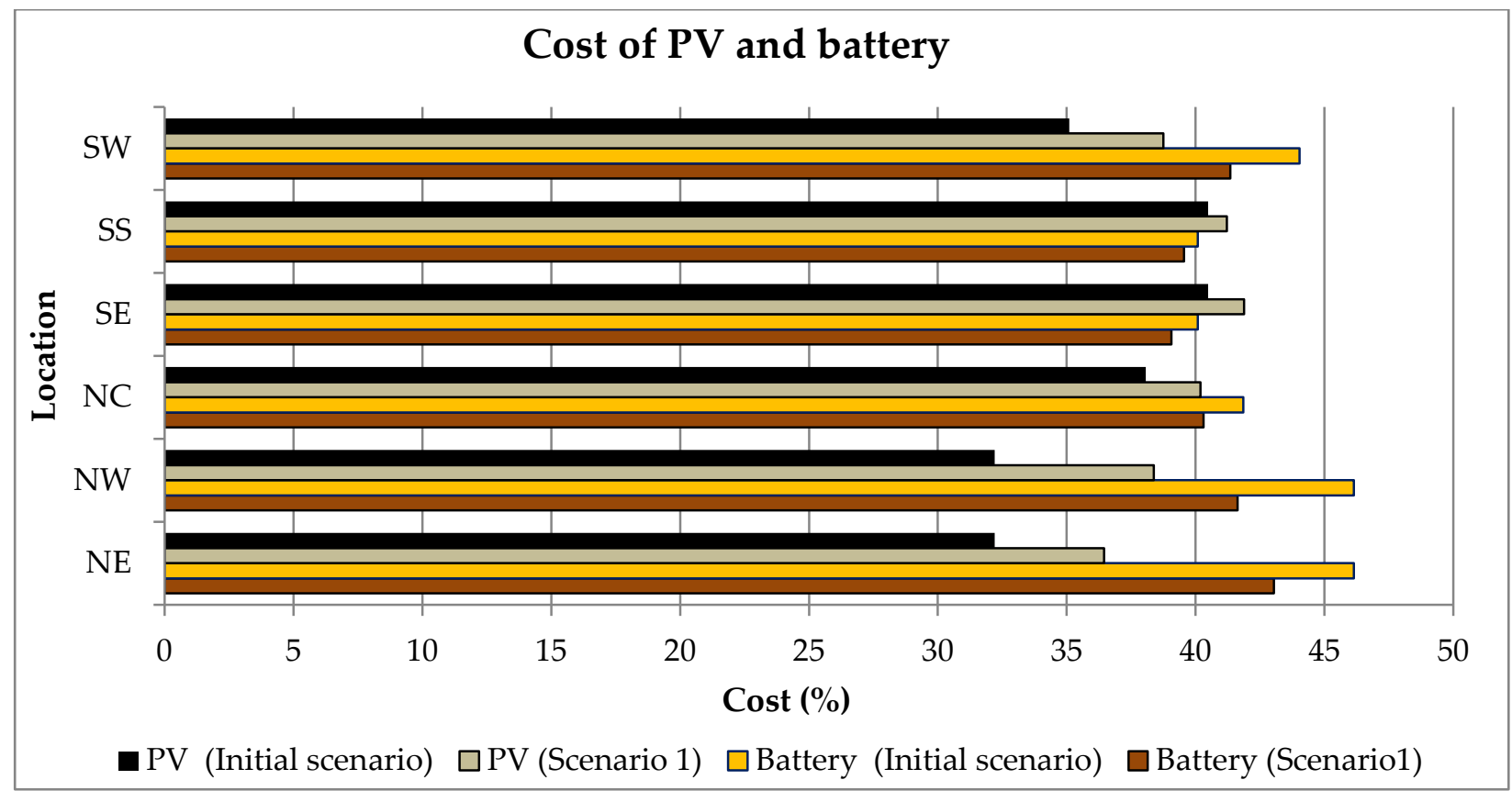

Figure 109: Percentage cost of the PV array and the battery bank

\section{1.3 Operation and maintenance and replacement costs}

The proposed SPM's operation and maintenance $(\mathrm{O}$ and $\mathrm{M})$ costs are presented in Figure 110. The $O$ and $M$ costs for the initial scenario are $\$ 3,049, \$ 3,049, \$ 3,361, \$ 3,510$, $\$ 3,510$ and $\$ 3,195$, respectively, while the values obtained for the scenario 1 are $\$ 3$, $269, \$ 3,380, \$ 3,490, \$ 3,601, \$ 3,557$ and $\$ 3,402$, for the specified zones.

The total replacement cost is $\$ 277,944$ and is the same for the electricity systems in all the zones. This is because the arrays will not be replaced during the project's useful life. Similarly, since the same battery bank is used for the initial scenario and the 
scenario 1, the same battery replacement cost of $\$ 237,256$ is incurred by the systems in all the zones. This value is $85.36 \%$ of the total system replacement cost and such a high value is obtained because the battery bank has a life span of $\sim 5$ years, meaning that it will be replaced four times over the 25-year project life. The replacement costs of the inverter and the LV capacitor account for the largest proportion of the remaining $14.64 \%$.

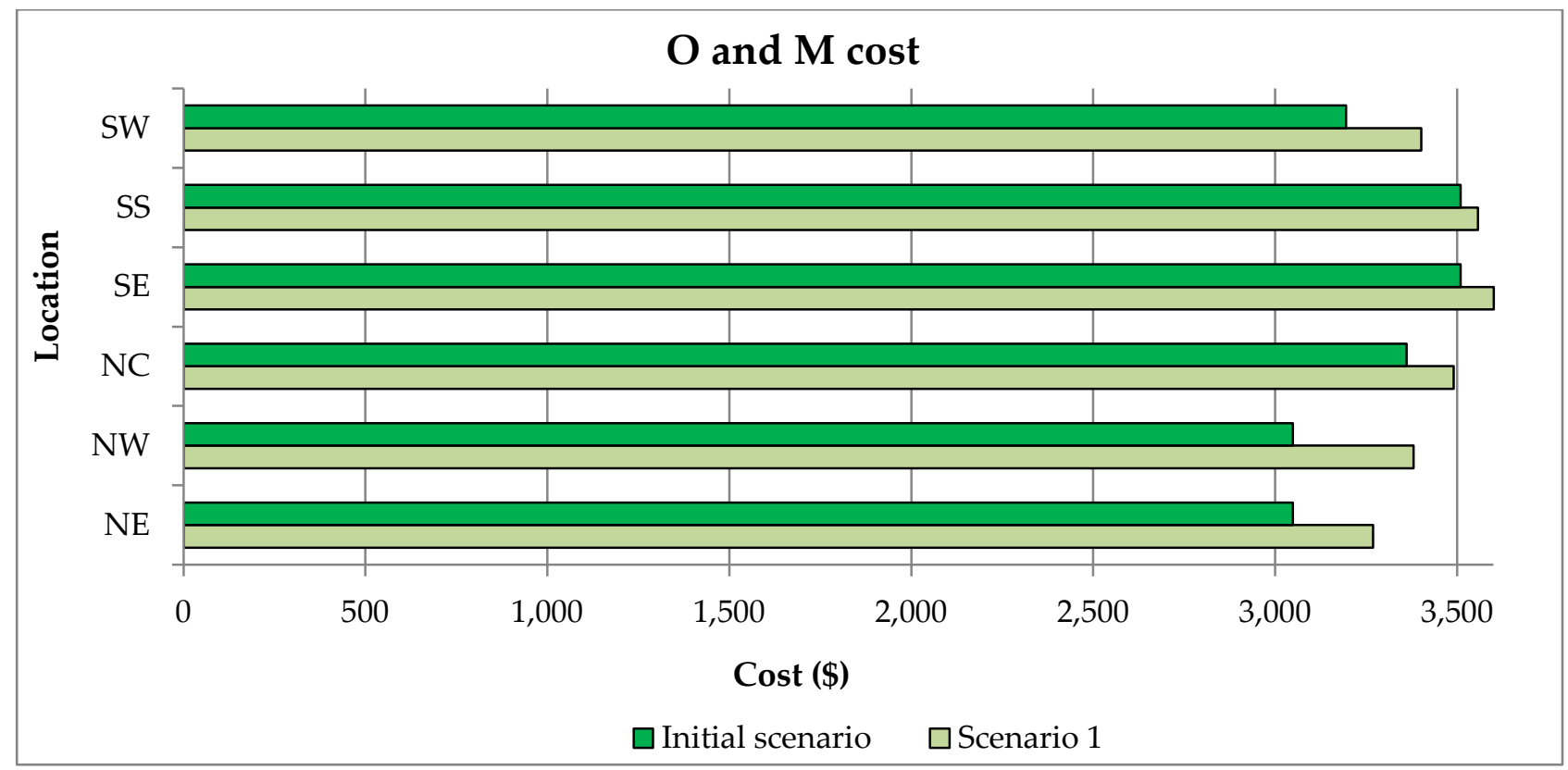

Figure 110: Operation and maintenance costs of the microgrids

\subsection{Life cycle cost}

The system's life cycle cost $(L C C)$ is shown in Figure 111. These have the same trend as Figures 108 and 110. Again, this is an indication that the LCC increases as the SPM's capacities are increased. The LCCs for the initial scenario are $\$ 425,491, \$ 425,500$, $\$ 440,595, \$ 447,789, \$ 447,800$ and $\$ 432,572$, for the specified communities, respectively. Similarly, the values obtained for the scenario 1 are $\$ 436,125, \$ 441,507, \$ 446,867, \$$ $452,222, \$ 450,086$ and $\$ 442,581$. The microgrid system in the SS zone has the highest capacity of $60.87 \mathrm{~kW}$ for the initial scenario, and it has the highest LCC. In the same vein, the microgrid system in the SE zone has the highest capacity of $65 \mathrm{~kW}$ in the scenario 1 and is also associated with the highest $L C C$. The results further show that the SPM's life cycle costs are $2.74-2.94$ times the initial capital costs. 


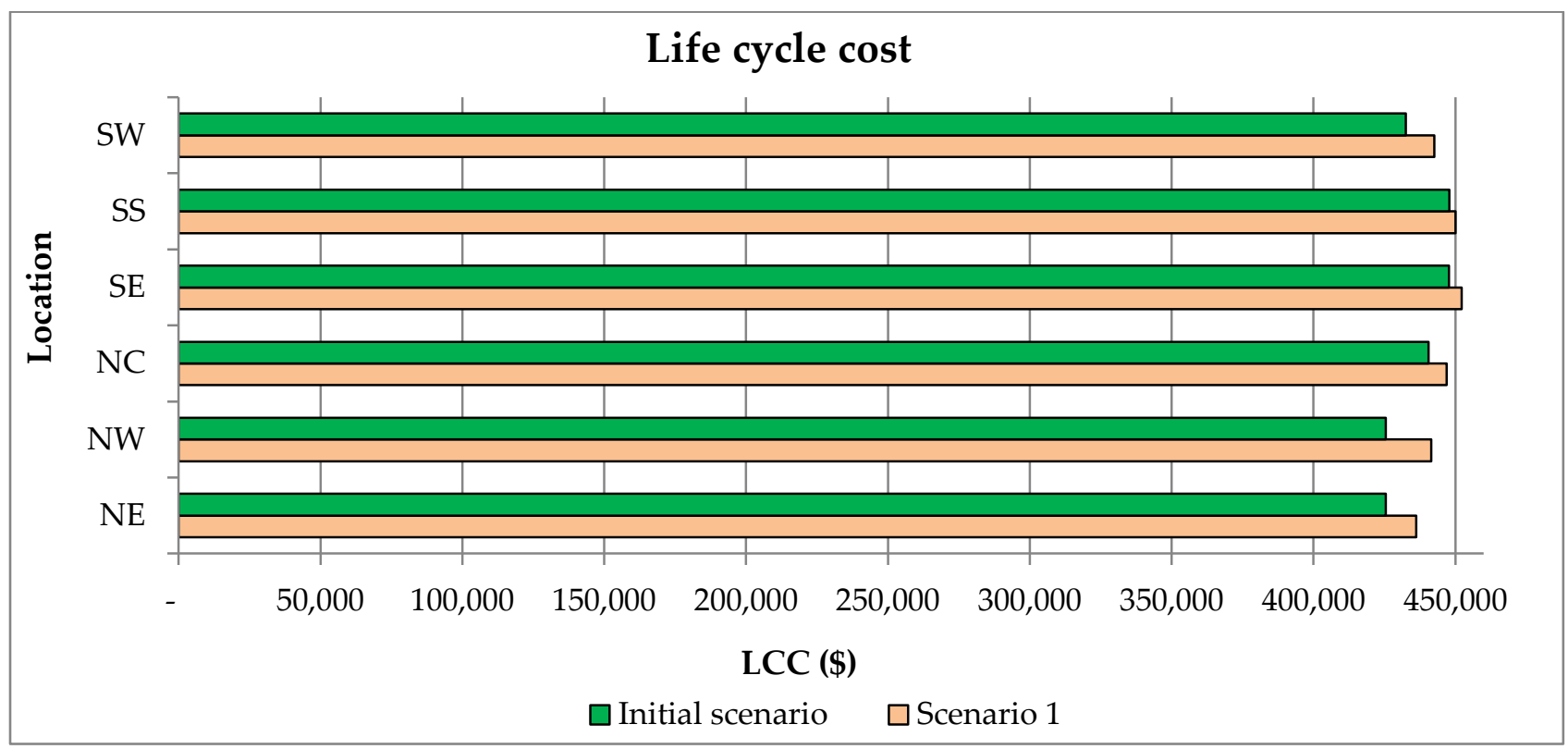

Figure 111: Life cycle costs of the photovoltaic microgrids

\subsection{Cost of energy}

The system's cost of energy (COE) is presented in Figure 112. The values of $\$$ 0.482/kWh, $\$ 0.494 / \mathrm{kWh}, \$ 0.436 / \mathrm{kWh}, \$ 0.409 / \mathrm{kWh}, \$ 0.483 / \mathrm{kWh}$ and $\$ 0.487 / \mathrm{kWh}$ are obtained for the initial scenario. The corresponding values of $\$ 0.396 / \mathrm{kWh}, \$ 0.377 / \mathrm{kWh}$, $\$ 0.400 / \mathrm{kWh}, \$ 0.388 / \mathrm{kWh}, \$ 0.470 / \mathrm{kWh}$ and $\$ 0.415 / \mathrm{kWh}$ are obtained for the scenario 1 .

The COE values obtained for the initial are higher than those of the scenario 1 because of the SPM's relatively lower energy production in the initial scenario. This generally supports the established fact, that the higher the useful energy delivered by the system, the lower the cost of energy per $\mathrm{kWh}[152,167,194]$. The lowest COE for the initial scenario is obtained for the SPM in the SE zone. However, the lowest value for the scenario 1 is obtained for the microgrid in the NW location, because of its relatively higher annual energy production. 


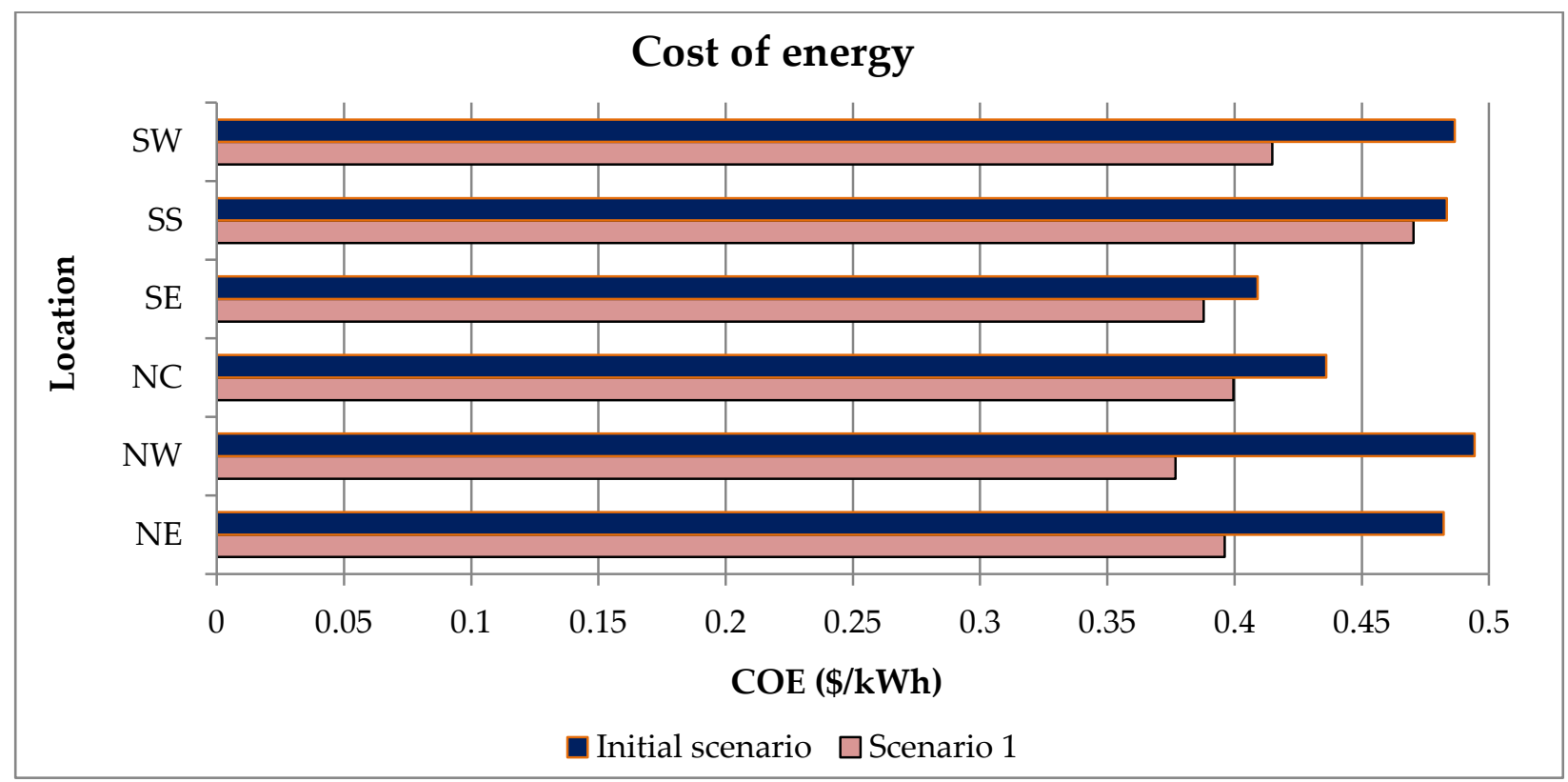

Figure 112: Cost of energy of the photovoltaic microgrids

\subsection{Cost of systems considering load growth}

\section{2. 1 Initial capital cost}

The SPM capacities are increased to manage the users' load demand growth, while the same battery bank capacity is still used. The system's initial capital costs for the increased demand scenario are presented in Figure 113. The microgrid systems in the NE zone have the lowest costs for all the users' demands. This is because the PV array sizes in the NE zone are lower than those of the other zones. These values are $\$$ $161,643, \$ 168,257, \$ 173,349$ and \$ 180,949 , respectively, for the $112.5 \%, 125 \%, 137.5 \%$ and $150 \%$ UD. However, the photovoltaic energy systems in the SE zone have the highest costs because of their relatively higher capacities. The costs are $\$ 179,177, \$ 187$, $748, \$ 194,036$ and $\$ 203,695$, respectively. The results reveal that the costs shown in Figure 113 are higher than those presented in Figure 108.

The results obtained for the $150 \% \mathrm{UD}^{*}$ and the $175 \%$ UD represent the system's costs for the $50 \%$ and the $75 \%$ users' load demand growth. In this case, the $150 \%$ UD $^{\times}$ and the 175\% UD are supported by the new PV array and battery capacities. In other words, both the PV array and the battery bank capacities are increased. The new battery size is $876 \mathrm{kWh}$, compared to the initial capacity of $540 \mathrm{kWh}$. The photovoltaic energy systems in the NE zone have the costs of $\$ 215,956$ and $\$ 229,219$, while the systems in 
the SE zone have the corresponding values of $\$ 238,702$ and $\$ 256,009$. Because the battery capacity is increased, the initial capital costs increase by $50-59 \%$.

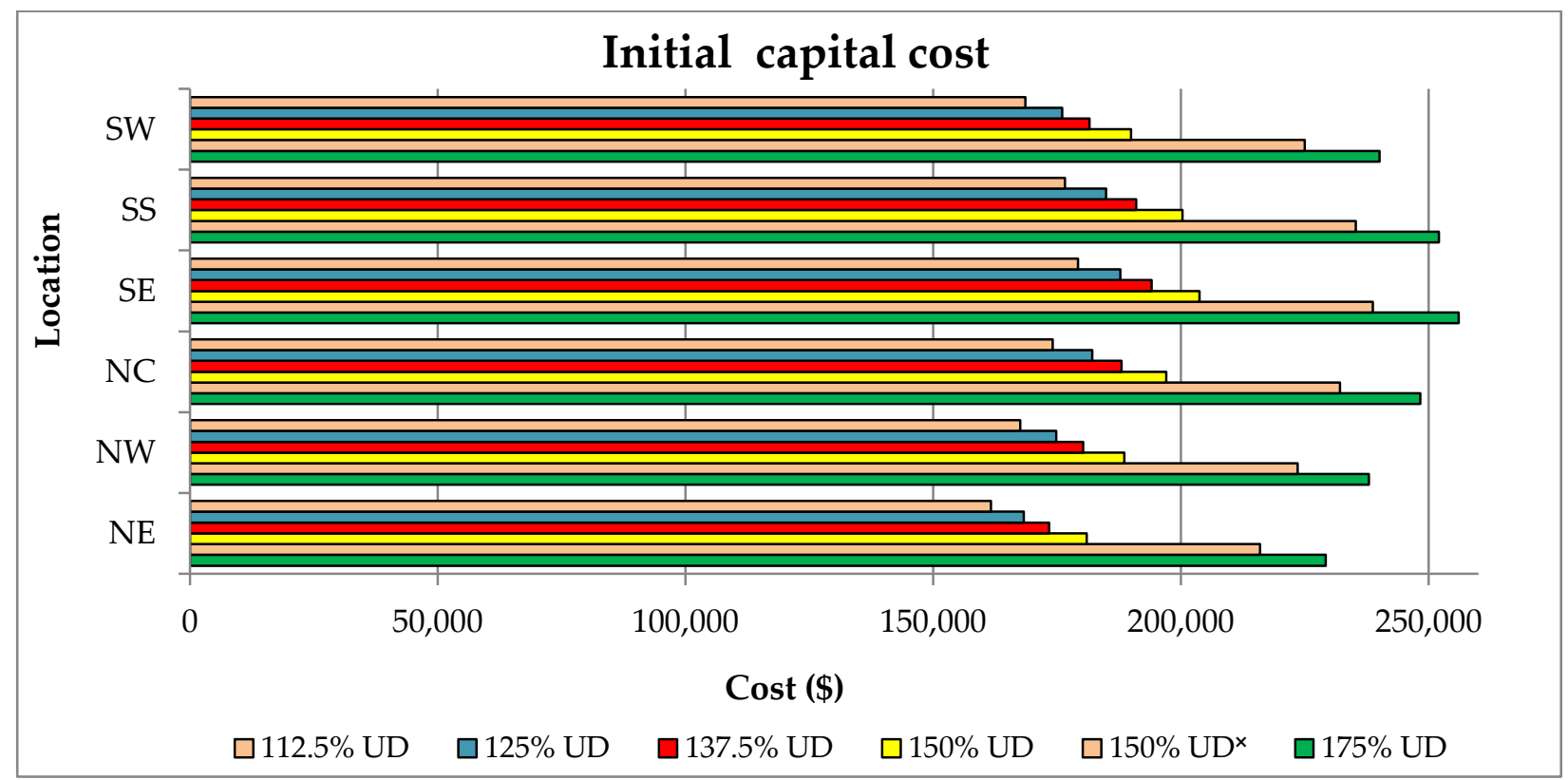

Figure 113: Initial capital costs for load growth scenario

\section{2. 2 Percentage costs of PV and battery components}

The percentage costs of the PV and the battery components are shown in Figures 114 and 115. The PV array costs for the 112.5 to $175 \%$ UD in the NE zone range from $\sim 38$ to $45 \%$ of the total initial capital costs. It is obvious that the systems supplying the $150 \%$ $\mathrm{UD}^{\times}$have the lowest costs. This does not, however, mean that the PV cost for the 50\% load growth is lower than the $12.5-37.5 \%$ load growth. The situation arises because the PV cost for the $150 \%$ UD scenario is the same with that of $150 \%$ UD $^{*}$, while the battery cost has increased by $\sim 67 \%$. The same explanation applies to the $175 \%$ UD. The respective costs in the SE zone range from $\sim 44$ to $47 \%$. However, the battery costs for the 112.5 to $175 \%$ UD range from $~ 33$ to $47 \%$ in the NE zones. 


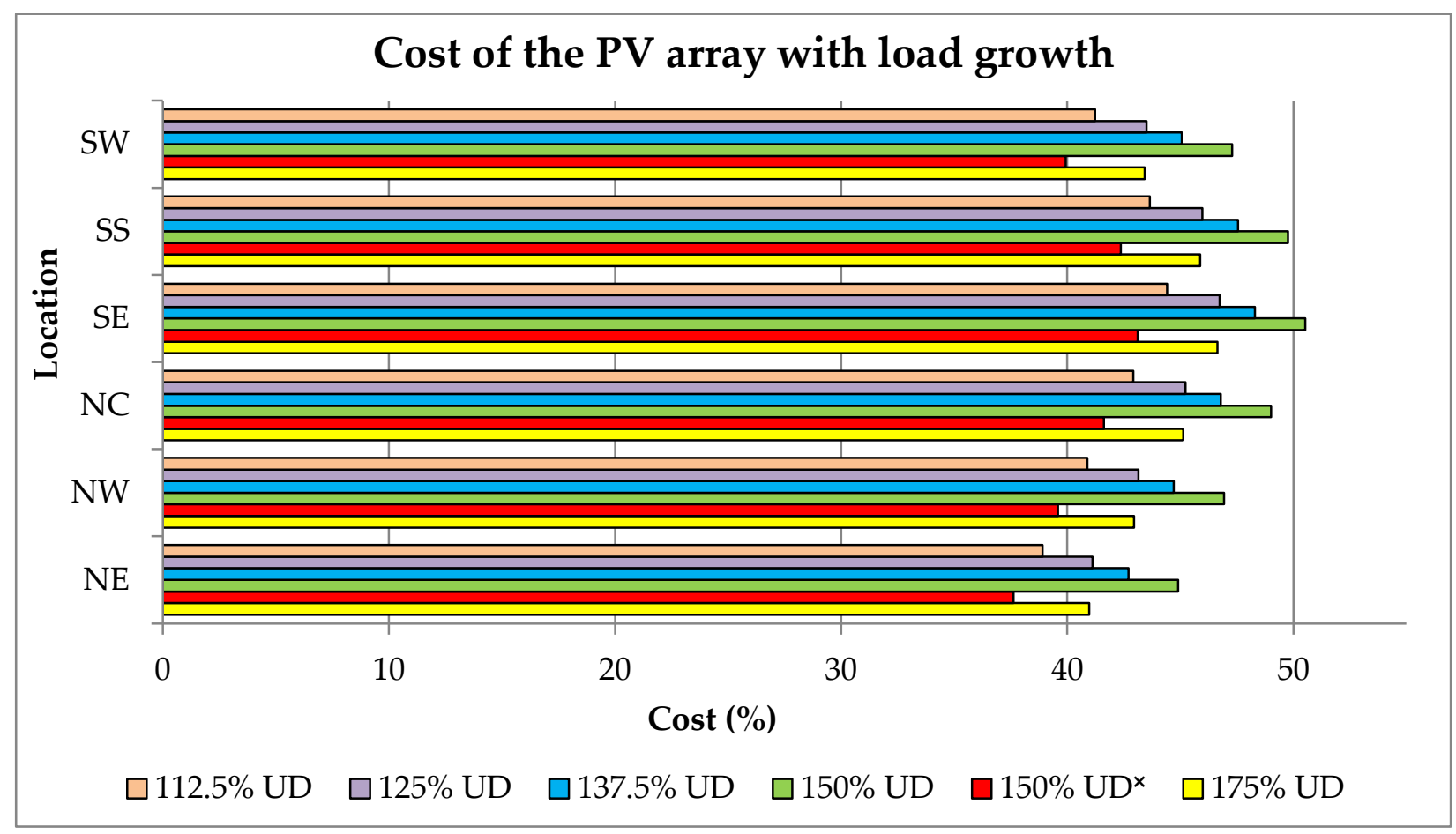

Figure 114: Percentage cost of the PV array with load growth

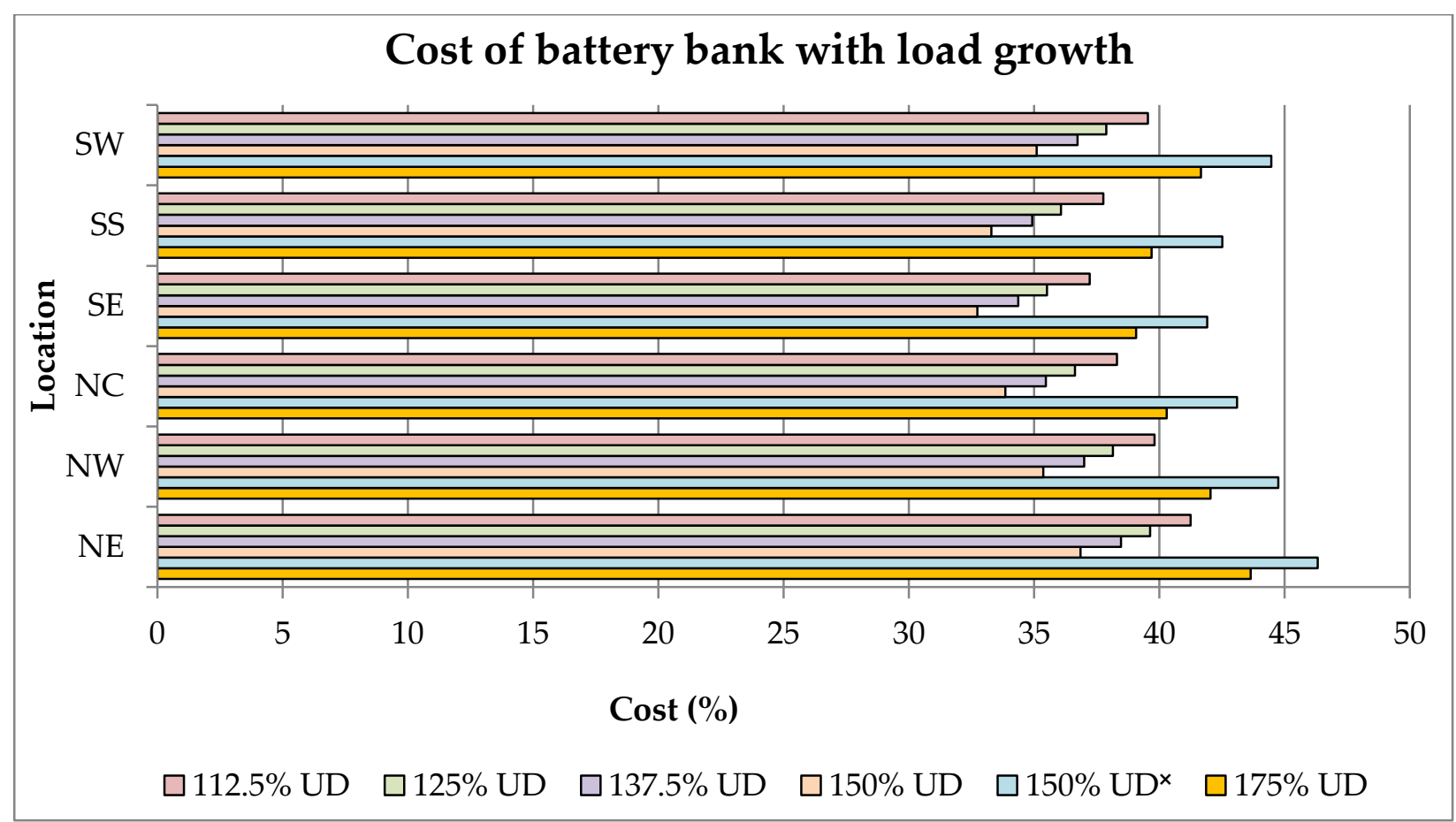

Figure 115: Percentage cost of the battery bank with load growth 


\section{2. 3 Operation and maintenance and replacement costs}

The $\mathrm{O}$ and $\mathrm{M}$ costs are shown in Figure 116 and they have increased compared to the values earlier presented in Figure 110. The results also show that the lowest values are obtained for the system in the NE zone. These are \$3,411, \$3,550, \$3,658 and \$3,818 for the $112.5 \%, 125 \%, 137.5 \%$ and $150 \%$ UD, respectively. The corresponding values obtained for the system in the SE zone are $\$ 3,558, \$ 3,715, \$ 3,830$ and $\$ 4,008$. The replacement cost of $\$ 277,944$ is obtained for all the systems, which is the same for the initial scenario and the scenario 1.

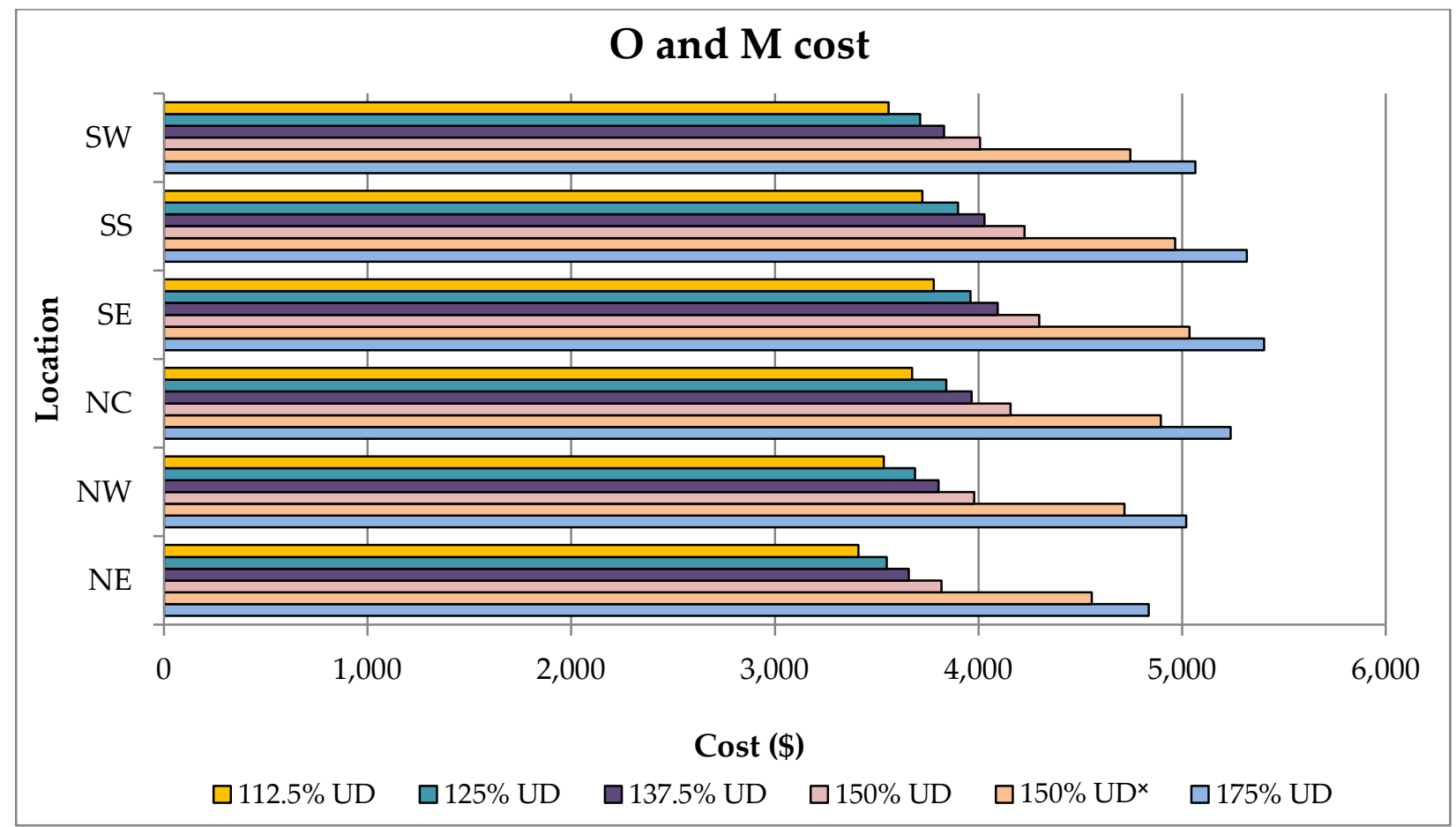

Figure 116: $\mathrm{O}$ and $\mathrm{M}$ costs for load growth scenario

\section{2. 4 Life cycle cost}

The life cycle costs presented in Figure 117 follow the same trend as those values presented in Figure 111. The lowest and the highest values are obtained for the systems in the NE and SE zones. The values are also higher than those presented for the initial scenario and scenario 1 in Figure 111. 


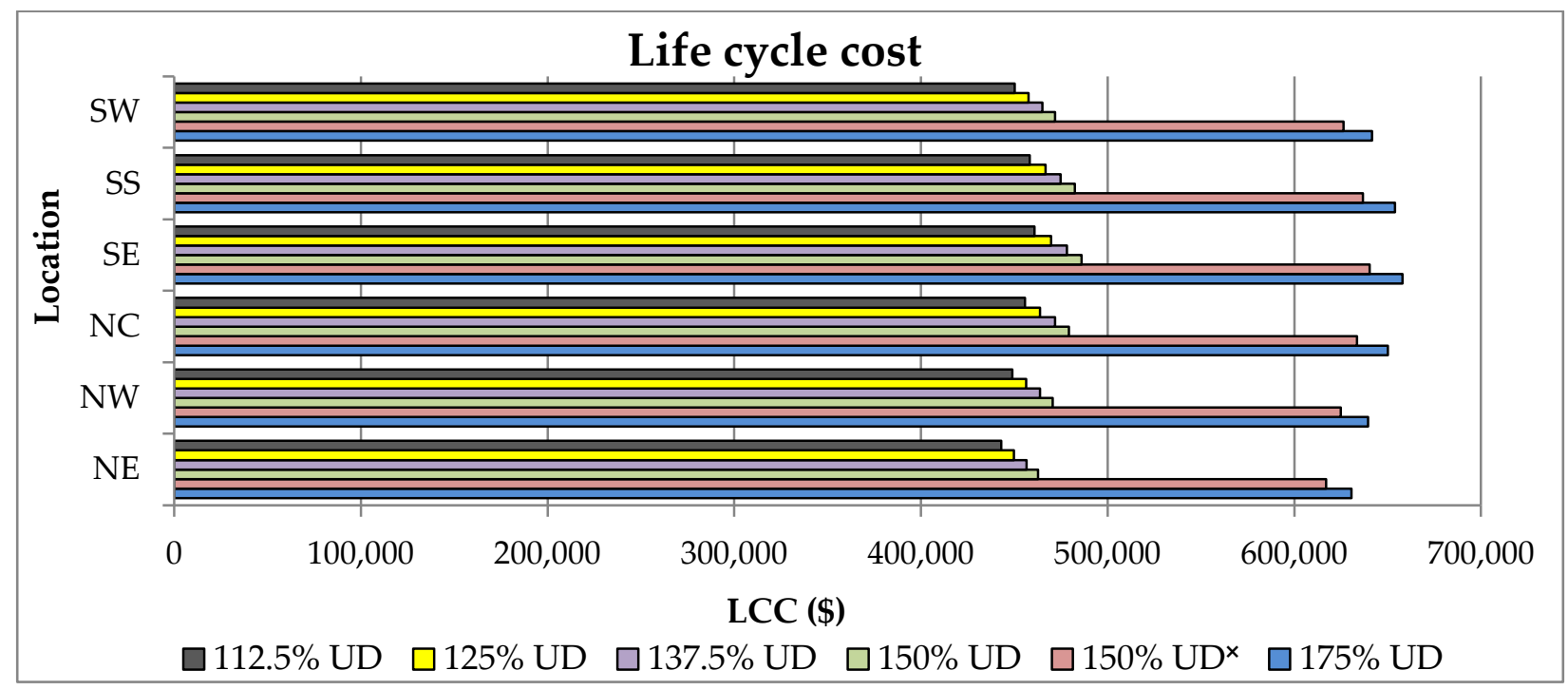

Figure 117: Life cycle costs for the load growth scenario

\section{2. 5 Cost of energy}

The cost of energy of the different load growth scenarios is shown in Figure 118. It can be clearly observed that the COE reduces as the solar PV microgrid capacities increase. This is attributed to the increase in the value of the useful energy produced by the systems. The literature establishes that the cost of energy has an inverse relationship with the useful electrical energy produced by the system [152, 167, 194]. The system in the SS zone produced the lowest energy for all the houses. Therefore, it attracts the highest COE compared to the other zones.

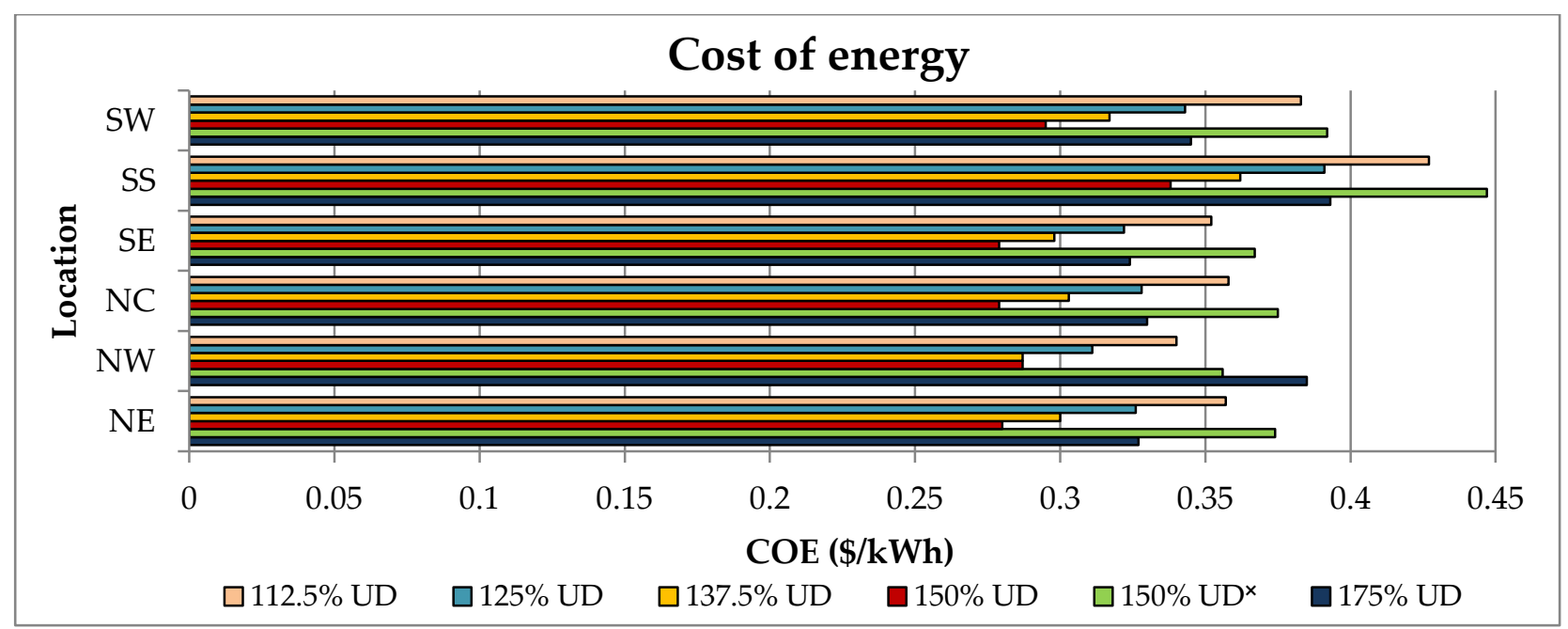

Figure 118: Cost of energy for the load growth scenario 
The results presented in Figures 113 to 117 clearly demonstrate that the initial, percentage cost of $\mathrm{PV} /$ battery, $\mathrm{O}$ and $\mathrm{M}$, replacement and the life cycle costs have increased for the 150\% UD " and 175\% UD. As earlier presented, the 150\% UD" and the $175 \%$ UD are supported by the new PV array and battery capacities. By increasing the number of strings from 2 to 3 , it is clear that the system's costs have drastically increased. For instance, the life cycle costs for the systems supplying the 150\% UD have been increased by $33.4 \%, 32.8 \%, 32.2 \%, 31.8 \%, 32.0 \%$ and $32.7 \%$ to obtain the values for the $150 \%$ UD for the specified zones. The replacement costs have also been increased from $\$ 277,944$ to $\$ 396,572$, which is $\sim 43 \%$ increase due to the additional battery string.

Increasing both the PV array and the battery bank in light of the load demand growth has the potential to improve the microgrid's availability. However, it is associated with high capital costs. A comparison of the reliability indices for the $150 \%$ UD $^{*}$ scenario in Figures 104 to 106 to the values earlier obtained for the 150\% UD in Figure 97 to 99 shows this clearly. The financial implications of increasing the battery capacity are shown in Figures 113 to 116.

Whilst it is important to consider the possibility of future load growth, it is also necessary to allow for trade-offs between the technical and the economic situations. This can help to achieve cost-effective energy solutions. One feasible way to accomplish this is by reducing the users' daily load demand. This could help to avoid the increase of the number of battery strings, while considering the future load growth. This possibility has been demonstrated with some results presented for the scenario 2 in Chapter 5. It is possible to allow a load growth up to $25 \%$ (i.e. equivalent to 6 additional houses) with increased SPM solar capacities and the initial battery size. This will lead to lower costs compared to the case of increasing the battery bank.

Additionally, since the users' loads are usually not classified as critical loads, they could be reduced at nights. This will require a relatively lower battery capacity, thus, reducing the overall system's initial costs [157]. The nocturnal energy requirement of the load profile in Figure 12 is $\sim 71 \%$ of the total users' daily demand. The fridges are operated as base loads in the analysis and they have the highest demand. Reducing the number of hours of operation of the fridges during the night could help to manage the SPMs with the locations' solar energy resources. Such consideration can be useful for achieving an economically feasible system. The results further demonstrate that 
achieving $100 \%$ availability by increasing the SPM's capacities is generally associated with very high initial, $\mathrm{O}$ and $\mathrm{M}$, replacement and life cycle costs. Therefore, reducing users' load demands can be used for realising a near $100 \%$ availability with relatively lower battery requirements.

This thesis considers 25, 50 and 75\% users' load demand growths of the baseline of $175 \mathrm{kWh} / \mathrm{d}$ (i.e. $63,875 \mathrm{kWh} / \mathrm{yr}$ ). For instance, the photovoltaic microgrid system designed for the $125 \%$ UD implies that the energy supply system will be required to support the users' total load growth of $25 \%$. In this case, the initial demand of 63,875 $\mathrm{kWh} / \mathrm{yr}$ will be increased to $64,514 \mathrm{kWh} / \mathrm{yr}$, over the SPM's useful life. The SPM capacities that can meet this energy consumption requirement are $63 \mathrm{~kW}, 69 \mathrm{~kW}, 76 \mathrm{~kW}$, $81 \mathrm{~kW}, 78 \mathrm{~kW}$ and $70 \mathrm{~kW}$, respectively, for the specified zones (see Figure 92). The energy production, yields, and the losses of these systems have been presented in Figures 100 and 101. The percentage increase in the SPM's capacities is $25 \%$ compared to the base case. The PV array capacities per house for the different locations are 2.63 $\mathrm{kW}, 2.88 \mathrm{~kW}, 3.17 \mathrm{~kW}, 3.38 \mathrm{~kW}, 3.25 \mathrm{~kW}$ and $2.92 \mathrm{~kW}$, respectively.

It is again noticed that the SPM's sizes of the communities depend largely on the location's characteristics - the solar irradiation cycles and the ambient temperatures, even though the same users' load demands are assumed.

\subsection{Comparison of SPM costs with a diesel plant cost}

A $30 \mathrm{kVA}$ diesel generator is selected for meeting the intended users' load demand requirements. The diesel power plant's fuel consumption is shown in Figure 119. These are 103.63, 109.01, 114.39, 119.78, and $125.16 \mathrm{l} /$ day for the 100 to $150 \%$ UD. The average consumption rates for the users' daily load demand range from 4.32 to 5.21 $1 /$ day. The fuel consumption is load following, which is why it does not scale linearly with the users' demand and it conforms to the users' load profile in Figure 12. This is because the useful hourly energy output of the generator is the energy that meets the users' hourly energy requirement [152]. However, it is clearly observed that the fuel consumption increases as the user's energy consumption increases from 100 to $150 \%$ UD. The analysis for each scenario has been done separately, and the results have been combined in Figure 119. 
The generator's initial capital, $\mathrm{O}$ and $\mathrm{M}$, and replacement costs are $\$ 26,562$, $\$ 5,538$ and $\$ 109,011$, respectively. The results reveal that the diesel's initial capital cost is $\sim 10$ to $17 \%$ of the SPM's initial costs. The generator's expected length of life is $\sim 4$ years. This means that it will be replaced five times during the 25-year project life. The diesel system's replacement cost is also $\sim 27$ to $40 \%$ of the values obtained for the photovoltaic microgrids.

The diesel plant's fuel and life cycle costs are presented in Figure 120. With Nigeria' diesel fuel cost of $\sim \$ 0.85 / 1$, the annual fuel costs range from 32,121 to $\$ 38,831$. The fuel costs over the 25-year system's life span range from 719,072 to $\$ 868,466$, while the life cycle costs range from 860,183 to $\$ 1,009,577$ for the 100 to $150 \%$ UDs. Though the generator's initial cost is lower than the values obtained for the SPMs, it has relatively higher life cycle and $\mathrm{O}$ and $\mathrm{M}$ costs (see Figures 108 and 113). The results further show that the diesel power system's life cycle cost is $~ 2-2.3$ times the SPM's life cycle costs for the six locations.

The diesel system's cost of energy for the 100 to $150 \%$ UDs is shown in Fig. 121. It ranges from 0.341 to $\$ 0.400 / \mathrm{kWh}$. The values are lower than those obtained for the solar photovoltaic microgrid systems. This is because of the diesel plant's relatively higher energy production. The higher the energy produced by an energy system, the lower is the cost of energy. Some of SPM's major benefits are that they are fueled by the sun and the solar PV arrays do not require any $\mathrm{O}$ and $\mathrm{M}$ and replacement costs over the project's lifetime, compared to the diesel-powered generation systems that have higher life cycle costs. The diesel power plant's life cycle cost is higher than those of the SPM systems due to the fuel and maintenance costs. This can be supported by the literature $[4,5,7,8$, $12,106,110,111,119]$.

When the users' load demand was analysed with a $15 \mathrm{kVA}$ generator, the cost of energy obtained was $\$ 0.46 / \mathrm{kWh}$. However, with a $30 \mathrm{kVA}$ generator, the cost of energy ranges from 0.341 to $\$ 0.400 / \mathrm{kWh}$. This signifies that the cost of energy reduces as the energy generation increases $[142,152]$. It is obvious that for the same scenario, a $15 \mathrm{kVA}$ generator produces a relatively lower energy for the users' requirements $[142,152]$. The $30 \mathrm{kVA}$ generator has been selected for the application according to the IEC standard 62257-7-3 [142], which guides the selection of generator sets for rural electrification 
systems. Since the energy system is off-grid, it is crucial to select a generator that can adequately support the users' requirements $[162,194]$.

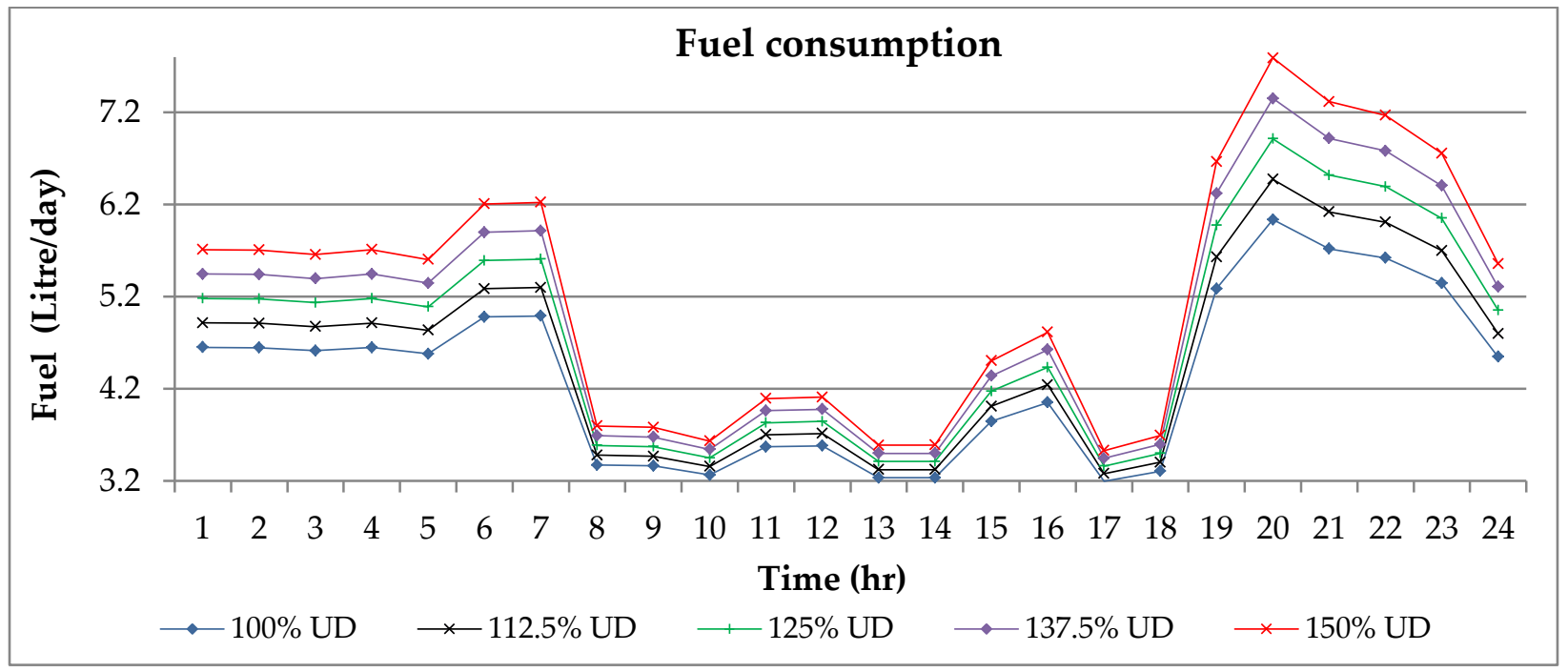

Figure 119: Daily fuel consumption for different demand requirements

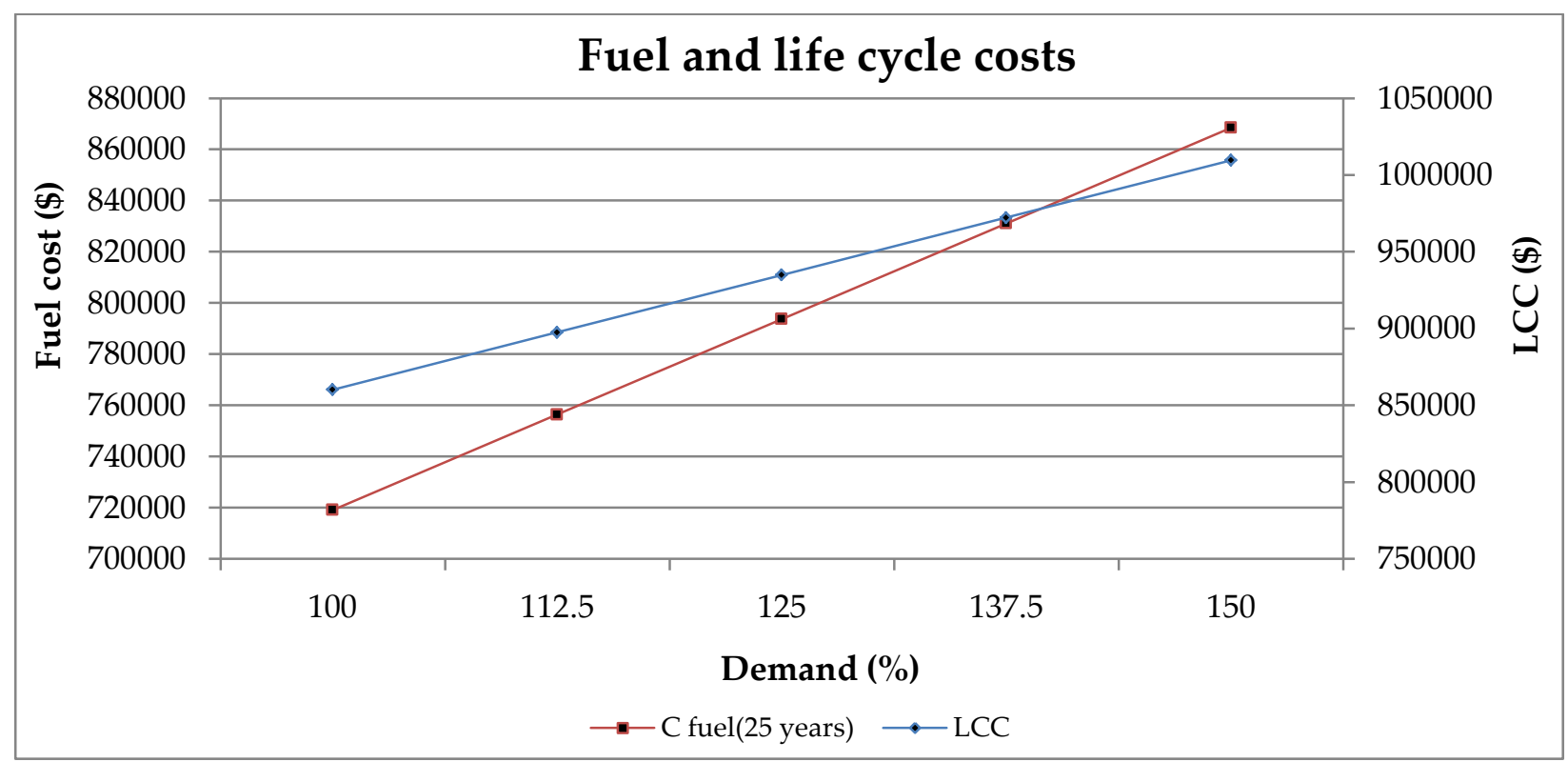

Figure 120: Fuel and life cycle costs of the diesel generator 


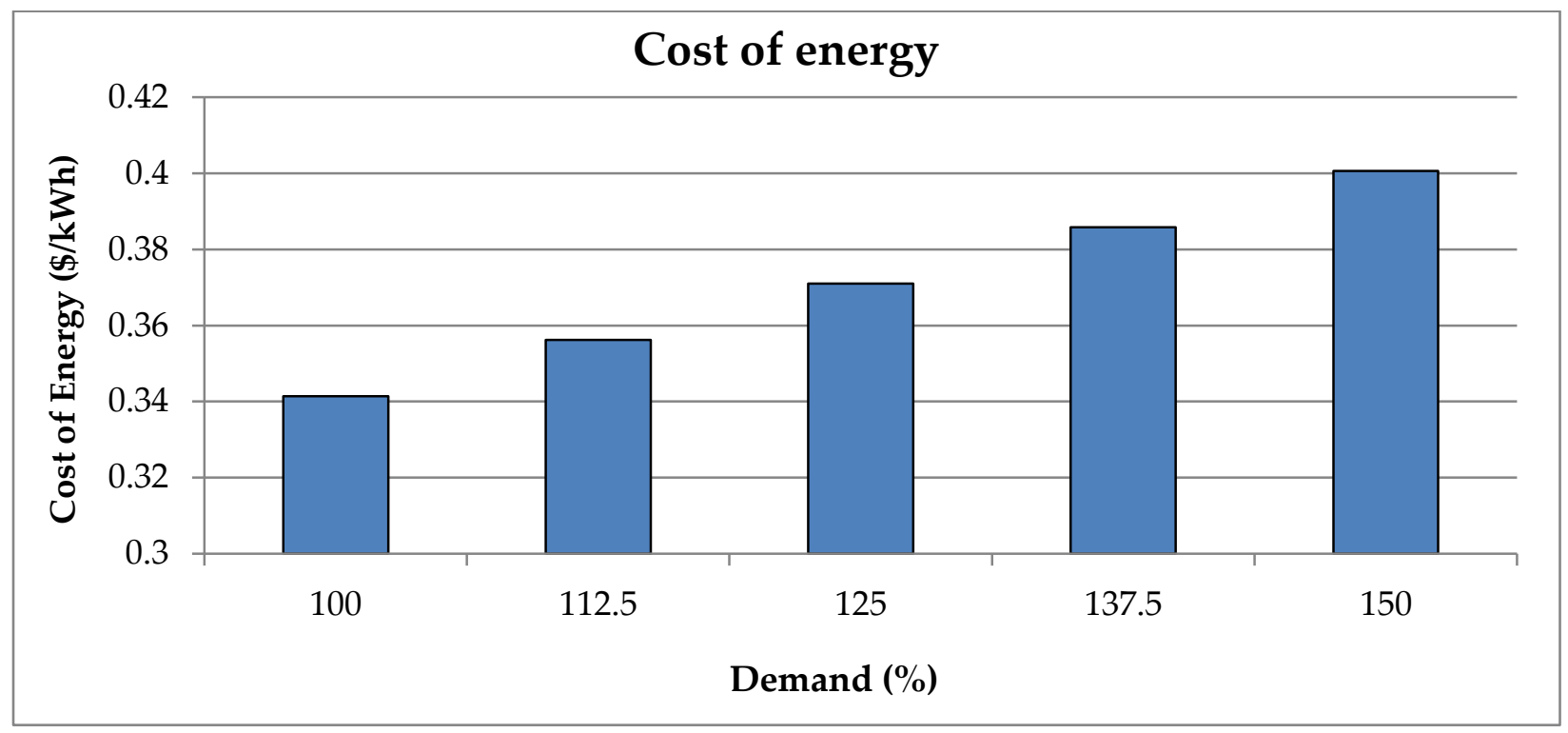

Figure 121: Cost per unit energy generation of the diesel power plant

\subsection{Chapter summary}

This chapter has primarily focused on a discussion of the SPM's economic performance with and without the users' load demand growth. The performance evaluation was done in terms of the initial capital, percentage cost of PV/battery components, operation and maintenance, replacement and the life cycle costs. The initial capital cost includes those of the PV arrays, battery, inverter/charger, capacitor, cables etc., and the installation cost. Even though solar photovoltaic arrays do not require any maintenance over their life span, a certain amount of money is spent on replacing and/or maintaining the other components like the battery bank, inverter, including cleaning the surface of the solar PV arrays.

The chapter demonstrates that though the generator's initial cost is lower than the values obtained for the SPMs, it has a relatively higher life cycle and $\mathrm{O}$ and $\mathrm{M}$ costs. The diesel generator's initial capital cost is $\sim 10$ to $17 \%$ of the values obtained for the SPMs. However, the diesel system's life cycle cost is about 2 to 2.3 times those of the proposed SPM systems.

Furthermore, the diesel generator is expected to be replaced five times during the 25 -year project life. The replacement cost is, therefore, $\sim 27$ to $40 \%$ of the SPM's replacement costs. However, because of the diesel plant's relatively higher energy 
production, its cost of energy for the 100 to $150 \%$ users' demand is lower than those obtained for the solar photovoltaic microgrid systems.

The SPM's array cost in the initial scenario ranges from 32 to $40.5 \%$ of the total initial cost, while the battery cost is $\sim 40 \%$ to $46 \%$. Similarly, the PV array cost in the scenario 1 ranges from $\sim 36$ to $42 \%$ of the total initial capital cost, while the battery cost ranges from $\sim 40$ to $43 \%$. This indicates that the PV modules and the battery costs constitute a significant portion of the SPM's initial capital cost. Again, this fact demonstrates the critical importance of ensuring the two components are carefully designed in order to achieve economically feasible PV microgrid solutions.

Yet, notwithstanding the diesel power plant's relatively lower initial capital cost, the solar photovoltaic microgrids have significant economic benefits. For instance, they are fueled by the sun and the solar PV arrays do not require any $\mathrm{O}$ and $\mathrm{M}$ and replacement costs over the project lifetime - a contrast to the diesel-powered generation systems that have higher life cycle costs because of the fuel and maintenance costs. The chapter has presented a detailed economic analysis of the proposed photovoltaic microgrid systems compared to the conventional diesel power plant. The SPM's environmental performance will be discussed in the next chapter. 


\section{Chapter 8}

\section{Environmental performance of the Microgrid}

This chapter discusses the environmental performance assessment of the proposed solar photovoltaic microgrids, both with and without the load demand growth. This is done by evaluating the quantity of carbon dioxide emissions avoided, life cycle emission rate, global warming potential (GWP), energy payback time (EPBT) and the energy return on investment (EROI) of the microgrid systems. The diesel system's fuel consumption reported in Chapter 7 is used to analyse the carbon emissions saved by the proposed microgrid systems. However, the emission rate reported in the literature for countries from which Nigeria imports its solar PV modules is used to obtain an estimated solar photovoltaic emission rate for the proposed microgrids. This emission rate is then used to evaluate the microgrid system's GWP for the specified zones.

The chapter is divided into three main parts. The first presents the analysis of the carbon dioxide avoided by the microgrid systems. The second estimates the microgrid's GWP by using the estimated life cycle emission rate. The third aspect reports the energy payback time (EPBT) of the photovoltaic microgrid systems in Nigeria's six geo-political zones. The fourth section focuses on the comparison of the environmental impact of the proposed microgrid systems with that of a diesel power plant. A summary concludes the chapter.

\subsection{Carbon dioxide emissions avoided}

Some diesel plant's emission factors have been reported in the literature. A value of $3.2 \mathrm{kgCO}_{2}$ per litre of diesel is reported in [195]; $3.15 \mathrm{kgCO}_{2}$ perlitre diesel [196] and $3.0 \mathrm{kgCO}_{2}$ per litre of diesel in [197]. An emission factor of $3.0 \mathrm{kgCO}_{2}$ per liter of diesel is used in this thesis based on the mentioned values.

The annual carbon dioxide emissions resulting from the fuel consumption for supplying 100 to $150 \%$ UD are presented in Figure 122. These range from 113.5 to 137 tonnes. The emissions are assumed as the quantity of carbon dioxide that can be saved by the proposed solar photovoltaic microgrids in the specified locations, according to 
the literature [202, 204]. The results, therefore, demonstrate that the higher the amount of fuel consumed, the higher the carbon dioxide emissions.

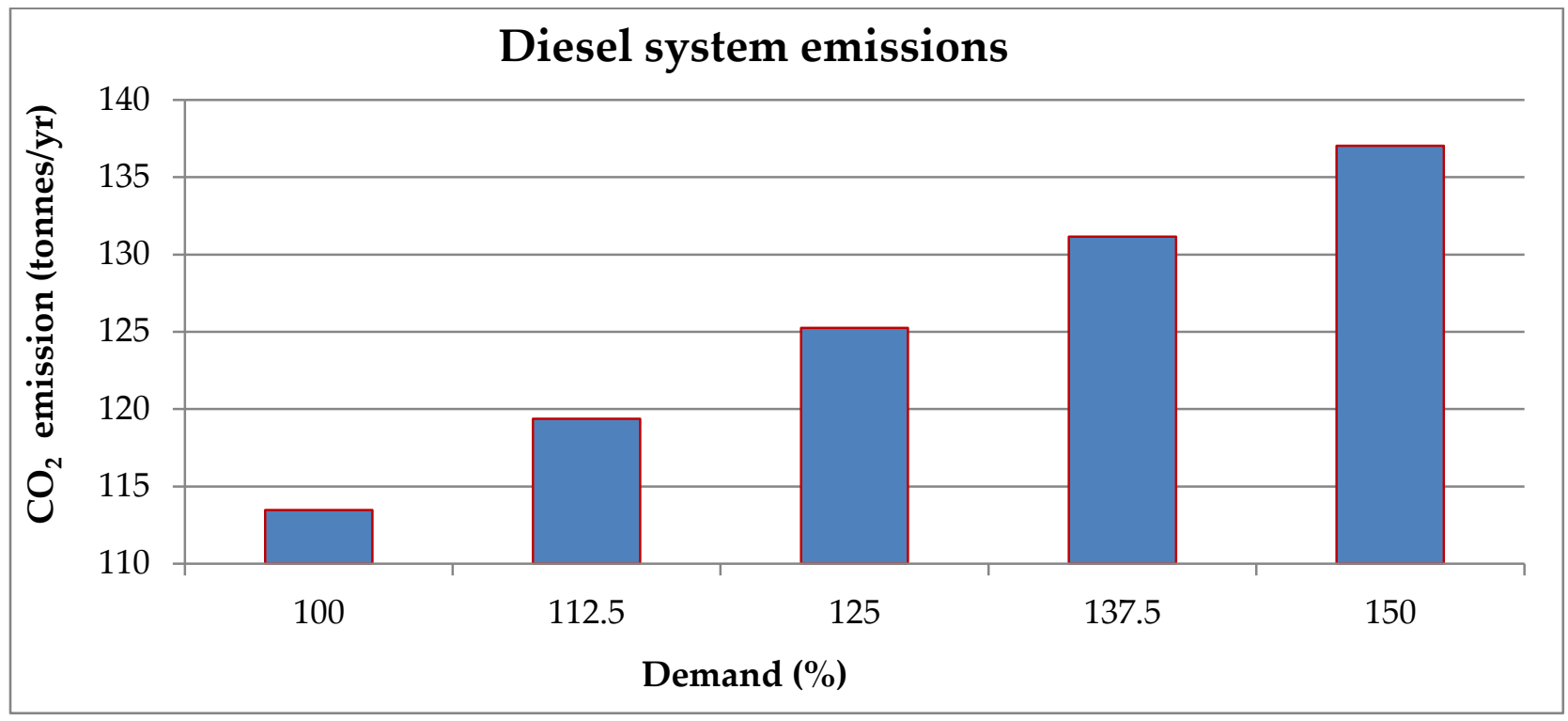

Figure 122: Annual carbon dioxide emission by the diesel power plant

\subsection{Emission rate and GWP of the proposed SPMs}

The $\mathrm{CO}_{2}$ emission rate is a useful index for evaluating the effectiveness of the solar photovoltaic systems in terms of global warming [129, 198, 158]. This index is usually measured in $\mathrm{gCO}_{2}$ per $\mathrm{kWh}$, and it is employed in this study to compare the proposed SPM's environmental impact with that of a diesel generator.

The life cycle GHG emissions for various technologies have been reported in [129], with crystalline PV systems having a value ranging from 50 to $100 \mathrm{gCO}_{2}-\mathrm{eq} / \mathrm{kWh}$. The lower emission values are obtained for the Southern European locations such as Spain and Italy, with a high solar irradiation of $1,700 \mathrm{kWh} / \mathrm{m}^{2} / \mathrm{yr}$. However, the higher emission values are obtained for the locations with relatively lower solar irradiation like Belgium, United Kingdom, and Germany.

The GHG emissions of $72.2 \mathrm{~g} \mathrm{CO}_{2}$-eq/kWh for mono c-Si PV modules that are manufactured in China have been reported in [130]. The authors used a solar irradiation of $1,700 \mathrm{kWh} / \mathrm{m}^{2} / \mathrm{yr}$ and module efficiency of $14 \%$. The life cycle emission ranging from 30 to $45 \mathrm{gCO}$-eq/kWh has also been reported in [199] for c-Si modules, using solar irradiation of $1,700 \mathrm{kWh} / \mathrm{m}^{2} / \mathrm{yr}$. Life cycle emissions ranging from $39-110 \mathrm{gCO}$-eq $/ \mathrm{kWh}$ 
has been presented for various solar photovoltaic modules under an average Switzerland solar irradiation of $1,100 \mathrm{kWh} / \mathrm{m}^{2} / \mathrm{yr}$ [200]. The life cycle emission values of 46 - 63 gCO - -eq $/ \mathrm{kWh}$ have been reported based on a solar insolation of $1,700 \mathrm{kWh} / \mathrm{m}^{2} / \mathrm{yr}$ [201].

This life cycle analysis information is used to obtain ballpark life cycle GHG emissions under the Nigerian conditions. From Figures 10, 13 and 14, the country's solar irradiation ranges from 1,490 to $2,224 \mathrm{kWh} / \mathrm{m}^{2} / \mathrm{yr}$ (i.e. from the southern to the northern zones), with an average of $1,923 \mathrm{kWh} / \mathrm{m}^{2} / \mathrm{yr}$. The solar irradiation of these locations is carefully considered and is used with an estimated emission rate of $\sim 57$ $\mathrm{gCO}_{2}$-eq/kWh to evaluate the system's global warming potential. With this information, the microgrid system's GWP for the different locations is presented in Figure 123.

The GWP values report the quantity of greenhouse gases (GHGs) emitted over the SPM's life cycle [129]. The values obtained for the six zones range from 3,409 to $7,945 \mathrm{kgCO}_{2}$-eq. These are comparable to the GWP of 6,000 $\mathrm{kgCO}_{2}$-eq reported for solar photovoltaic systems in [129]. The results reveal that the impact of the proposed solar photovoltaic microgrid in one location is conspicuously different from the other, even though the locations are in the same country. This is due to the disparities in the locations' solar irradiation and the amount of electricity produced by the proposed microgrids.

Realistically, the amount of electricity produced by the systems is influenced by the location's solar insolation, losses due to temperature, dust, inverter conversion etc. These parameters are different for the locations. The results presented in Figure 123 demonstrate that the locations with higher solar irradiation have relatively lower impacts both for the initial scenario and the scenario 1. This knowledge can be supported by the analysis presented in [129] that the regions with a relatively higher solar irradiation have lower GHG emissions. Therefore, the photovoltaic microgrids in the NE, NW and SW regions have lower global warming potential compared to those in the NC, SE, and SS. The lowest and the highest GWP values of 3,409 and 7, $975 \mathrm{kgCO}_{2}-$ eq are obtained for the photovoltaic systems in the NE and SS regions, respectively. 


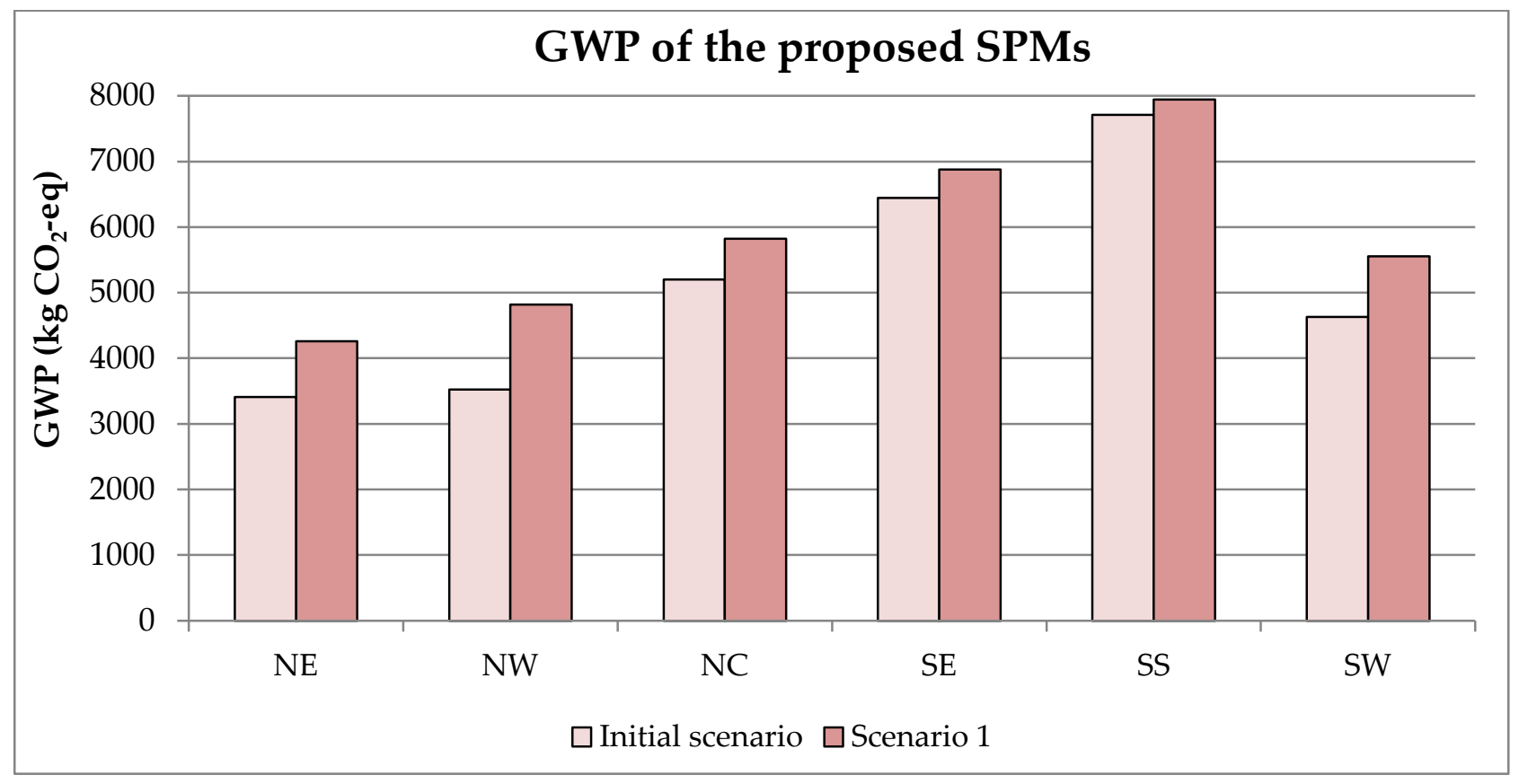

Figure 123: The proposed SPM's GWP

\subsection{Energy payback time}

The cumulative energy demand (CED) of 4,350 MJ-eq. is used to evaluate the proposed system's EPBTs over the 25-year lifetime. This value is the average of the CED of 3,800 and 4,900 MJ-eq. reported in $[129,130]$. With $\eta_{8}$ of $30 \%$, the energy produced per solar photovoltaic module and the EPBTs for the SPMs are shown in Figure 124.

The values of EaGEN per module in the NE, NW, NC, SE, SS and SW zones are 1,178, 1,148, 994, 961, 814 and 1,015 MJ, respectively. These translate to 327, 319, 276, 267, 226 and $282 \mathrm{kWh}$ for the specified zones. The SPM's EPBT for the locations is about $1.11,1.14,1.31,1.36,1.6$ and 1.29 years. The results reveal that the energy system with the highest energy generation has the lowest EPBT, while the one with the lowest energy production has the highest EPBT. These are the NE and the SS locations, respectively. Again, the disparities in the values of EPBT are attributable to the difference in the locations' solar energy potential [158]. With this analysis, it can be concluded that it will take between 1.11 and 1.6 years for the proposed solar photovoltaic systems to pay back the energy used in their life cycles.

The EPBTs of $0.5-2$ years are reported in [211]. The author also highlights the factors on which the payback time depend, such as location, solar irradiation and the 
technology used. The EPBTs of about 1.9 and 2.4 years are reported in [130] for mono cSi technologies in Europe and China, respectively. In addition, values of $1.7-2.7$ years have been reported in [198]. The literature also reports EPBTs of 1.38, 1.45, 1.50, 1.49 and 1.51 years [214] for Philippines, Europe, Korea, Malaysia, and the USA. The EPBT of $1.11-1.6$ years obtained in this study can be compared with the mentioned values.

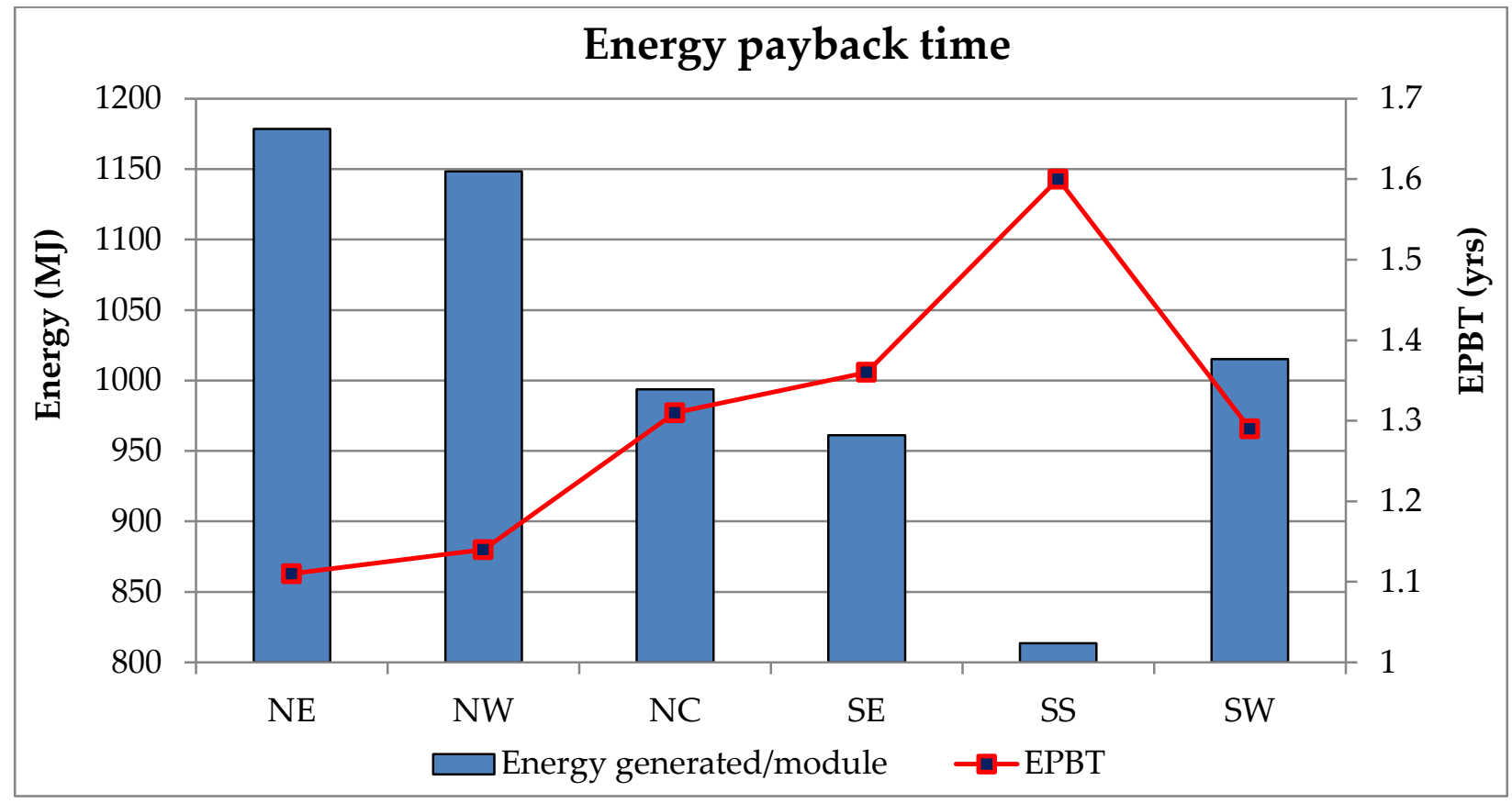

Figure 124: The energy payback time of the SPM

\subsection{Energy return on investment}

The EROIs for the zones are shown in Figure 125. These range from 12.5 to 18.02, 15.63 to 22.52 and 18.75 to 27.03 , for lifetimes of 20,25 and 30 years, respectively. Though this study assumes a 25-year project lifetime, the analysis is also done for 20 and 30 years to achieve a wide range of results. The values signify that the electricity systems have the potential to produce many times as much energy over their life cycle, as it takes to manufacture them. This indicates that if the proposed microgrid systems are implemented, they can offer significant environmental benefits of producing more energy than consume over their life cycle. The results also demonstrate that EROI increases with lifetime, according to Eq. (48) [129]. The lowest and the highest values are obtained for the 20 and 30 years, respectively. 
Furthermore, it is obvious that the energy system in the NE location has the highest EROI, while the one in the SS location has the lowest EROI. Again, the disparities in the values of EROI are attributable to the difference in the zones' solar energy potential. Figure 125 shows that the EROIs for the proposed solar photovoltaic microgrids are proportional to the amount of energy they produced per module. The implication of this relationship is that those solar photovoltaic systems having relatively higher energy generation have higher values of EROI.

However, Figure 124 demonstrates that the energy payback times are inversely proportional to the amount of energy generated per module. This indicates that the solar photovoltaic microgrid systems with relatively higher energy generation have lower values of energy payback time. Therefore, it is desirable to achieve higher and lower values of EROI and EPBT, respectively.

The energy return on investment values of 16.1 and 12.6 were reported in [130] for solar electricity systems in Europe and China, respectively. Also, a value of 12 has been reported for a solar photovoltaic system in Spain [129]. The literature also reports EROIs of about 21.5, 20.8, 19.6, 19.6 and 19 for Philippines, Europe, Korea, Malaysia, and the USA [214]. These were obtained under the mean USA solar irradiation and system life span of $1,800 \mathrm{kWh} / \mathrm{m}^{2} / \mathrm{yr}$ and 30 years, respectively. The EROIs of 18.75 27.03 obtained for a lifetime of 30 years in this study, can be compared with the mentioned values. It can be observed that the values of 27.03, 26.32, 22.9, 22.06 and 23.26 obtained for NE, NW, NC, SE and SW zones, respectively, are higher than the values reported for the Phillipines, Europe, Korea, Malysia and the USA. The value of 18.75 obtained for the SS zone is lower than the values reported for these countries. 


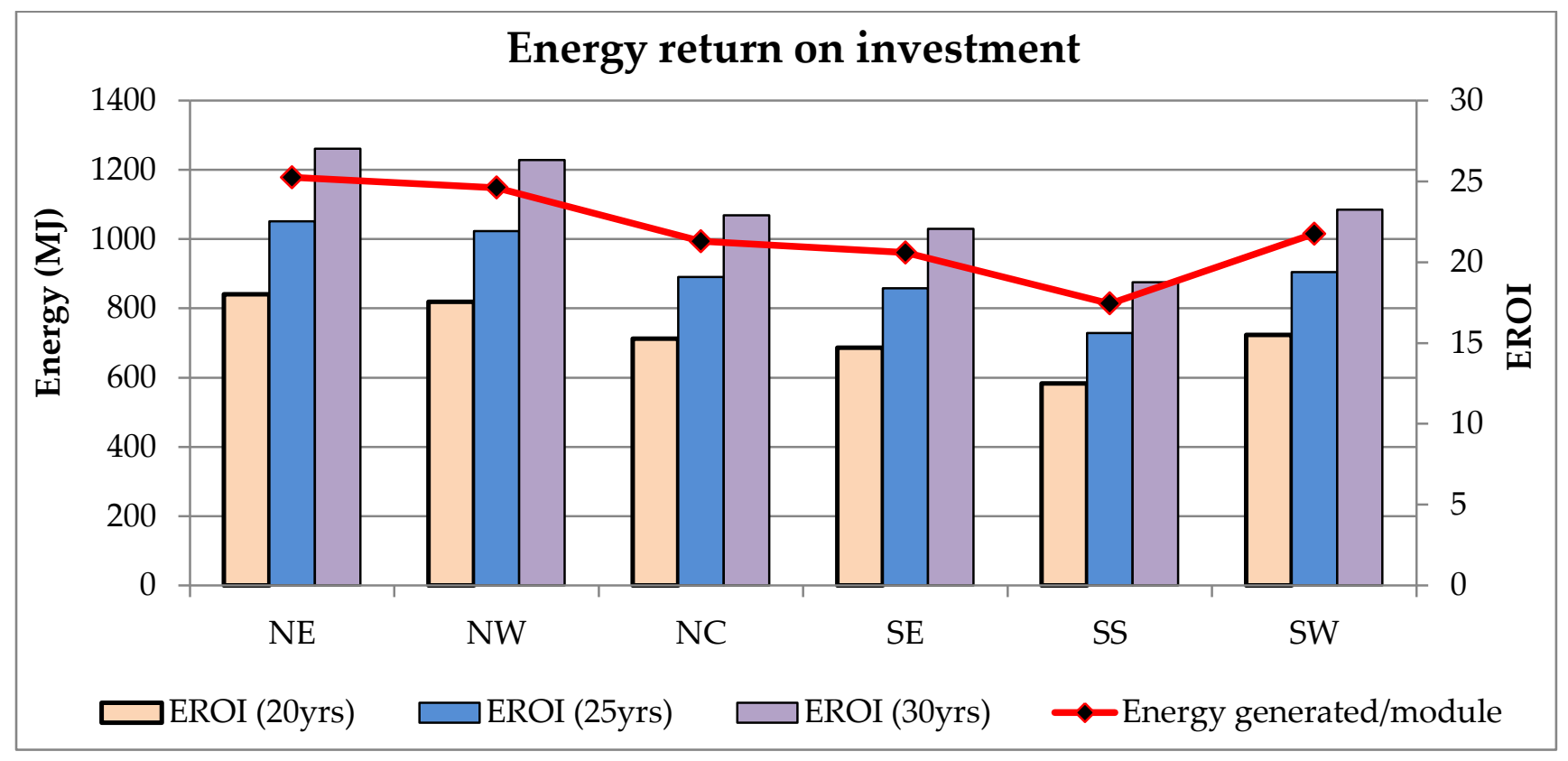

Figure 125: The energy return on investment of the SPMs

\subsection{Comparison of impact of SPM systems with a diesel system}

The diesel power plant's $\mathrm{CO}_{2}$ emission rates range from 576 to $695 \mathrm{gCO}_{2} / \mathrm{kWh}$ for $100-150 \%$ UD. The values of 576, 606, 636, 665 and $695 \mathrm{gCO}_{2} / \mathrm{kWh}$ are obtained for 100 , $112.5,125,137.5$ and $150 \%$ UD, respectively. These can be compared with the 870 $\mathrm{gCO}_{2} / \mathrm{kWh}$ reported in [202], $763 \mathrm{gCO}_{2} / \mathrm{kWh}$ in [203] and $1,178 \mathrm{gCO}_{2} / \mathrm{kWh}$ in [204]. These values are very high compared to the estimated SPM's emission rate of 57 $\mathrm{gCO}_{2} / \mathrm{kWh}$, and those other values reported in the literature. The National Renewable Energy Laboratory (NREL) also reports that the life cycle emission rates of renewable electricity systems are lower than those of conventional fossil-fueled power plants by $400-1,000 \mathrm{gCO}_{2} / \mathrm{kWh}$ [215]. The SPMs clearly have the potential to mitigate global warming due to their relatively lower carbon emissions [129].

Figures 119 and 122 give information about the amount of fuel and carbon emissions assumed to be saved by the proposed microgrid systems. The analysis indicates that it is possible to avoid a significant amount of carbon dioxide emissions are over the project's life span, if the photovoltaic microgrids are implemented. This thesis has taken some steps further than most related existing studies [103-128, 177-186], by looking closely at the aspects of microgrid design, planning and performance 
evaluation. It has also examined the $\mathrm{CO}_{2}$ emission rate and the GWP for PV systems, and also comparing them with those of the fossil-fuel based system.

The commonality of most of the related studies is the assumption that the total carbon dioxide emissions by a fossil-fuel based system are the same as the amount of the emissions avoided by the solar PV system. Importantly, however, some carbon dioxide gases are emitted when the PV modules are being manufactured and this makes the solar technologies not $100 \%$ carbon neutral. The fact that solar PV systems are not without emissions are demonstrated by some existing studies [129, 130, 190, 197203].

Life cycle assessments (LCAs) are a complex evaluation that requires extensive studies. Approximate emission rate and cumulative energy demand values have been used in this thesis to assess the proposed SPM's life cycle impact, compared to the conventional diesel energy system. This technique has been employed because there is no solar photovoltaic cell manufacturing system in Nigeria, and as such, there is no existing study on LCA for the country [158]. However, future work will consider the evaluation of the life cycle impact of the solar photovoltaic modules that are fabricated at the NASENI's 7.5 MW Solar Panel Manufacturing plant. This is the only existing solar module plant in the country and it produces its PV modules from imported solar cells.

\subsection{Chapter summary}

This chapter has mainly focused on a discussion of the environmental performance of the different solar photovoltaic microgrid systems with and without the users' load demand growth. This was done by analysing the carbon dioxide emissions avoided, the proposed system's life cycle emission rate, global warming potential, the energy payback time and the energy return on investment.

With an emission factor of $3.0 \mathrm{kgCO}_{2}$ per litre of diesel, the fuel consumed by the diesel plant ranges from 103.6 to $125.2 \mathrm{l} / \mathrm{d}$ for the 100 to $150 \%$ users' demand. This is used to calculate the quantity of carbon dioxide emissions produced by the generator, which range from 113.5 to 137 tonnes .

Furthermore, an estimated life cycle emission rate of $\sim 57 \mathrm{gCO}_{2}$-eq per $\mathrm{kWh}$ was used to obtain the system's GWP ranging from 3,795 to 7,765 $\mathrm{kgCO}_{2}$-eq. The chapter 
also demonstrates with results that the impact of a solar photovoltaic microgrid in one location is conspicuously different from systems in the other locations. This is due to disparities in the solar irradiation of the zones and the amount of electricity produced by the proposed systems.

The diesel plant's $\mathrm{CO}_{2}$ emission rates were discussed for different users' demand (UD). These range from 576 to $695 \mathrm{gCO}_{2} / \mathrm{kWh}$. In addition, these values are very high compared to the SPM's value of $57 \mathrm{gCO}_{2} / \mathrm{kWh}^{\prime}$. It can be concluded that solar photovoltaic electricity systems have very low carbon emission rates compared to the diesel power system.

The SPM's energy payback times for the specified locations are about 1.11, 1.14, $1.31,1.36,1.6$ and 1.29 years. The results show that the energy system with the highest energy generation has the lowest EPBT, while the one with the lowest energy production has the highest EPBT. These locations are Bununu in the NE zone and Amurukeri in the SS zone, respectively. Again, the disparities in the values of EPBTs are attributable to the difference in the solar energy potential of the six different locations. This information indicates that it will take between 1.11 and 1.6 years for the proposed solar photovoltaic modules to pay back the energy used in their life cycles.

The chapter has reported EROIs of 22.52, 21.93, 19.08, 18.38, 15.63 and 19.38 for the solar photovoltaic microgrids for the six location. These values signify that the microgrid systems have the potential to produce 15.65 - 22.55 times as much energy, over their 25-year lifetime, as it takes to manufacture them. Clearly, the proposed solar microgrids, producing more energy than they consume over their life cycles, can offer significant environmental benefits.

Approximate values have been used to indicate the proposed SPM's life cycle carbon emissions and global warming mitigation potential, compared to the conventional diesel energy system. The other environmental performance indicators have also been presented such as the energy payback time and the energy return on investment. These provide a better environmental impact assessment compared to some existing work in the literature. 


\section{Chapter 9}

\section{Conclusions and recommendation}

This chapter concludes the thesis by reflecting on the key findings, making recommendations and providing directions for future research. The research examined the techno-economic and environmental analysis of solar photovoltaic-based electrification systems for remote households in different locations. Some of the communities that do not currently have an electricity access in Nigeria are used as case studies. To achieve this prime goal, the following questions have been carefully considered. These questions address the following;

- How to determine the appropriate electricity generation capacity that is able to adequately support the basic energy requirements of a specified community?

- How a solar photovoltaic microgrid system (SPM) can be depended on for meeting the users' load demand for every hour of the day?

- How reliable will the microgrid system be with and without load growth?

- To what degree is the photovoltaic microgrid system economically and environmentally viable?

The first question fundamentally refers to the fact that a community combines different kinds of people, with different preferences, appliances and the energy usage patterns. These are coupled with the possibility of seasonal variations in the users' energy consumption. The second question refers to the intermittent characteristic of the intended communities' solar energy resources. Also, the third question centers on the solar power plant's reliability with and without the users' load demand growth. The last question focuses on how the solar PV systems can compete with the conventional energy system from the perspective of cost and environmental impact.

The questions have been answered by introducing approaches that are based on the global engineering standards and practical experience. This includes assessing the users' daily energy needs and usage patterns, designing and modelling stand-alone SPMs with capacities that can balance the community's energy consumption. The strategies also consider evaluating the proposed system's performance over the dry and the rainy seasons during the year. This was then used to analyse the system's 
availability with and without future load demand growth. The proposed solar power system's environmental performance was also examined. A comprehensive literature review was conducted for the solar photovoltaic technologies, energy storage systems and the decentralised photovoltaic electricity systems for developing countries. This presents the state-of-the-art and provides the opportunity to identify the knowledge gaps in the literature.

Furthermore, a general system planning strategy was discussed. This divides the off-grid energy project into four phases, such as the pre-design, detailed design, implementation and the post-implementation stages. This thesis focuses on the first and the second phases. The effectiveness of the proposed photovoltaic energy systems is evaluated by comparing the thesis results with that of a diesel energy generation system, and with the findings in the relevant literature.

\subsection{Achieved Objectives}

This thesis has achieved the following objectives:

- It assesses the intended users' daily energy needs and the usage patterns in the specified communities and creates an aggregate load profile for 24 households per community.

The author obtained the daily energy consumption and pattern of the individual houses through a preliminary assessment of the users' lifestyles. This information was first evaluated and then further processed to create the community's aggregate load profile, by adding the load demands of the 24 different houses. Through this analysis, information about the users' daily peak and off-peak electricity demands was also obtained. The 24-hour load profile thus serves as the working demand profile for all the six different communities that are used as case studies in the thesis.

- It designs and models stand-alone SPMs with electrical generation capacities that can balance the energy usage of the households in the communities.

The aggregate load profile that was employed for designing and modelling the appropriate SPMs that could support the intended users' energy requirements, in relation to the locations' solar energy resources. The microgrids for the six locations were simulated under different environmental factors, i.e. solar irradiance and ambient temperature. This provided a means to quantitatively 
ascertain the disparities in the solar photovoltaic power capacities for the different localities.

A suitable battery energy system was also sized, modelled, and included in the SPM model for balancing the mismatch between the load profile and the intermittent solar energy generation. The energy demand versus energy generation simulations and analyses were done for the whole year. Such analyses provide useful knowledge and information about how the variability of the energy source and the users' load demands could be managed over the 8760hour operation time.

- It evaluates the system's performance during different seasons - dry and rainy seasons and assesses its loss of energy probability and availability over the year, with and without load growth.

The SPM's performance analysis has been achieved by detailed assessments of the possible energy generation and the associated losses over the year. Introducing the derating factor, the temperature coefficient of power, the load reduction scenario, and the SPM capacity increase to the energy models gave rise to interesting performance results.

The performance indices are presented in terms of the power output, energy production, normalised yields and losses and the efficiency. The normalised yields and losses provide a performance summary suitable for comparing the proposed SPMs with different solar photovoltaic energy configurations or designs operating in other locations. The SPM's reliability over the year was also evaluated in terms of the unmet load demand, loss of energy probability and the availability indices. This, done both for the SPMs with and without load demand growth, thus provided a general knowledge about the confidence level at which the microgrid systems in the different locations can support the users' load demand requirements, under different load conditions.

- It conducts comparative cost and environmental benefit evaluations for the proposed SPMs and the diesel energy option.

The SPM's economic and environmental performances were quantitatively assessed. A life cycle cost analysis (LCCA) technique was used to evaluate the economic performance of the different SPM capacities. This is compared to the LCCA of the conventional diesel power generation system. The results show a wide range of cost differences between the solar photovoltaic microgrids and the 
diesel energy system over the assumed project lifetime of 25 years. These costs are presented in terms of the initial, operation and maintenance, replacement and the life cycle costs, as well as the cost of energy generated.

A life cycle impact assessment was used to assess the SPM's environmental performances. This was first presented in terms of the annual fossil fuel saved, $\mathrm{CO}_{2}$ emissions avoided, emission rate and the global warming potential compared to the diesel system. The SPM's emission rate was estimated based on the existing values reported in the literature and then compared with the diesel power plant's emission rates. The proposed system's EPBTs and EROIs are also determined. The results of the analysis were then compared with those reported in the literature to evaluate the achieved objectives.

\subsection{Contributions}

This section presents the major conclusions based on the thesis' contributions. Solar photovoltaic power generation technologies can be used to develop clean, costeffective and reliable electricity supply systems for remote communities in developing countries. Practical approaches, guided by relevant global engineering standards, are necessary for proposing suitable photovoltaic microgrid systems for different locations. The microgrid design strategies and the techno-economic and life cycle impact analysis that are introduced in the thesis have achieved comprehensive results of academic value and interests. This can be used for a better understanding of how to plan and develop distributed photovoltaic power systems for off-grid applications in developing countries.

\section{2. 1 Proposing a practical model}

A practical microgrid model is proposed in Chapter 4 that is based on a community's layout comprising 24 houses, the users' lifestyles and the solar energy resource. The layout is found to be useful because it provides relevant information about the physical arrangement of the households within the community. This includes the distances between the houses and the proposed SPM that help determine the actual lengths of cables required to supply the generated electricity to the houses from the photovoltaic power plant. Identifying the actual lengths of the conductors is useful for estimating the line losses. The common domestic appliances such as compact 
fluorescent lamps, fans, TVs, radios, fridges etc. constitute the electrical loads in the proposed model.

In addition, the community's aggregate load profile has been developed based on the users' energy consumption patterns. The existing local factors such as the current users' energy status and the available solar energy resource are also instrumental in understanding the problem and how to provide possible solutions. The knowledge and information of the geography and the communities that are used as case studies, then informs the proposed techno-economic design and analysis, rather than completely relying on assumptions. Furthermore, the proposed model will be useful for strengthening the local capacity, as it considers the possibility of using locallyfabricated solar photovoltaic modules. This contribution has been published as part of the following journal articles:

(1) Akinyele D. O, Rayudu R. K, Nair N. K. C, “Development of photovoltaic power plant for remote residential applications: The socio-technical and economic perspectives", Applied Energy 2015; 155: 131-149.

(2) Akinyele D. O, Rayudu R. K, “Community-based hybrid energy supply system: A practical and comparative approach", Applied Energy 2016; 171: 608-628

\section{2. 2 SPM capacities through design safety factor}

Chapter 3 of the thesis proposes the use of design safety factor $\left(d_{s f}\right)$ to determine the SPM's rated capacities. This factor is location-specific and unique. It is ascertained through a detailed solar photovoltaic performance evaluation based on the required design parameters, such as the users' highest daily demand, peak sun hours and the location's solar irradiance, solar irradiance at STC, rated PV array maximum power at STC, derating factor, temperature coefficient of power of the PV array, actual PV array temperature and the array temperature at STC.

Sizing the SPMs by using the $d_{s f}$ is found to be useful because it provides a practical means of determining different solar PV capacities for a particular community of interest. Such system ratings will adequately support the users' load demand requirements while also compensating for the losses. Once this factor is obtained for a particular system in a specified location, it can be used to determine the size of every other solar photovoltaic system in the same location. 
Furthermore, using this technique has allowed a wide range of SPM sizes to be determined. This saves the time that could have been spent by using the sizing methodology to estimate several SPM system's capacities, one after the other (see Chapter 5 for the results). The factors can be used as benchmark over-rating factors for any solar photovoltaic application in a specified site. From the practical point of view, solar photovoltaic modules are over-rated so as to compensate for losses and then provide enough current to charge the battery.

Therefore, the $d_{s f}$ introduced in this thesis is found to be reliable because it has been quantitatively obtained rather than being assumed. Apart from this advantage, it also provides a better and easy understanding of the fundamental solar photovoltaic microgrid design. This is because the SPM's sizes are easily determined by multiplying the $d_{s f}$ with the system's ideal capacity (see Chapter 3 ). Such a sizing technique is thus useful for practical photovoltaic system application. This contribution has been published as part of the following papers:

(1) Akinyele D. O, Rayudu R. K, “Community-based hybrid energy supply system: A practical and comparative approach", Applied Energy 2016; 171: 608-628

(2) Akinyele D. O, Rayudu R. K, Rodney H. G. Tan, “Comparative study of photovoltaic technologies based on performance, cost and space requirement: Strategy for selection and application", International Journal of Green Energy 2015. DOI: 10.1080/15435075.2015.1112287

\section{2. 3 SPM performance indices}

The thesis highlights with results in Chapter 6 the usefulness of rigorous battery SoC analysis in determining how the intermittent energy produced by the SPMs has been managed over the year. It finds that relatively lower and higher battery SoC is proof of lower and higher solar irradiation cycles of the specified locations, respectively. This is helpful when making decisions and planning, e.g. when deciding whether to introduce load reduction or to increase the SPM's capacity during the periods of low SoC. Apart from this, introducing the battery SoC probability distribution in the microgrid's performance assessment is also found to be useful in determining whether or not the battery bank receives enough charging current from the system.

The thesis proposes a detailed SPM performance evaluation method that is useful for a better academic understanding of photovoltaic system applications. This method can be used to predict the possible system's energy generation and losses in different 
localities. Accounting for PV energy losses makes the method better than some existing related research results in the literature that do not consider energy losses. The thesis finds that a performance analysis that considers losses is helpful for appropriately ascertaining what is likely to be the proposed SPM's behaviour and outputs under realworld operating conditions.

By introducing the derating factor and the temperature coefficient of power into the SPMs' energy generation model, the research is able to quantify the energy losses as a result of the ambient temperature, incomplete absorption of solar irradiation and the inefficiencies of the balance of system (BOS). This is useful for achieving realistic system outputs, as discussed in Chapter 5. Furthermore, examining the system performance in terms of the normalised yields and losses is beneficial for comparing the proposed SPMs with different photovoltaic systems in other locations.

The thesis findings also indicate that the normalised yields and losses of different SPM sizes in the same location are the same, and are different from the values obtained for the microgrid systems at other locations. This is useful for identifying an error or inconsistency in the performance analysis of different solar PV systems in a particular location. The SPM with the lowest losses and the highest efficiency is found in a location having the lowest solar irradiance of all the six locations. This reveals that the performance of the solar photovoltaic systems is dependent on the location.

The thesis finds that the proposed SPMs can provide a reliable electricity supply for the intended localities. The results presented in Chapter 6 show this can be determined through some reliability indices, such as the unmet load demand, loss of energy probability, and the availability. This helps to understand the relationship between the system's intermittent energy generation, users' load demands, and the battery SoC. It also helps to determine the extent to which the proposed SPMs could be reliable.

Integrating the system performance analysis with the reliability assessment has been shown with results to be a valuable means of evaluating the effectiveness of the proposed energy models. The thesis results show the practicality of the proposed models by comparing the system design, performance and reliability assessments of the proposed SPMs with and without the users' load demand growth. This comparison is needed because of the possibility of the users' energy consumption increase, which needs to be carefully considered during the design and planning stage. Such an analysis that considers users' load growth provides a better academic knowledge on the 
development of stand-alone energy systems for off-grid applications (see Chapter 7). This contribution has been published as part of the following papers:

(1) Akinyele D. O, Rayudu R. K, "The techno-economic and life cycle environmental performance analyses of a solar photovoltaic microgrid system for developing countries", Energy 2016; 109: 160-179

\section{2. 4 Economic performance of the proposed systems}

The thesis analysis underscores that the proposed SPMs have the potential to offer cost-effective electricity solutions for the communities, compared to the conventional diesel system. It finds that the diesel energy system's life cycle costs are more than twice as those of the SPMs, even though the diesel plant's initial capital cost is as low as 15 to $18 \%$ of the values obtained for the SPMs (see Chapter 7). The relatively higher diesel system's life cycle cost is attributable to the fuel and maintenance costs over the 25-year project life span.

The results also demonstrate that the initial capital costs of both the PV and the battery systems constitute the largest percentage of the SPM's total initial capital costs. These values range from $\sim 75$ to $82 \%$ of the total initial capital costs. This information can be useful for identifying the cost-effective energy configurations for the communities. This contribution has been published as part of the following papers:

(1) Akinyele D. O, Rayudu R. K, “Community-Based Hybrid Energy Supply System: A Practical and Comparative Approach", Applied Energy 2015; 171: 608-628

(2) Akinyele D. O, Rayudu R. K, "The techno-economic and life cycle environmental performance analyses of a solar photovoltaic microgrid system for developing countries", Energy 2016; 109: 160-179

\section{2. 5 Life cycle environmental performance of the proposed systems}

Chapter 8 highlights that using a life cycle assessment (LCA) technique to evaluate the SPM's environmental performance presents better results than some existing studies in the literature. For example, a comparison of the SPM's life cycle $\mathrm{CO}_{2}$ emissions rate with that of a diesel energy system identifies that the SPM system's emission rate is as low as $\sim 8.2-9.9 \%$ of the values obtained for the diesel generation system. The results show that the SPM's global warming impact is very low compared to that of the conventional diesel system. 
Furthermore, the environmental performance assessment helps to estimate the amount of fossil fuel that could be saved and the carbon dioxide emissions that could be avoided by the proposed solar photovoltaic microgrids. The EPBT and the EROI evaluations are also useful for determining the time required for the proposed photovoltaic energy systems in the six different locations to pay back the energy used in their life cycles, and how many times they will be able to generate the primary energy used for their manufacture.

The thesis also reports that LCAs depend on location, environmental impacts of the manufacturing of the PV technology (e.g. mono c-Si), the energy produced by the PV systems and their lifetimes. This information could be helpful for understanding and quantifying the environmental impact of photovoltaic technologies compared to the conventional diesel energy technology. This contribution has been published as part of the following papers:

(1) Akinyele D. O, Rayudu R. K, "The techno-economic and life cycle environmental performance analyses of a solar photovoltaic microgrid (SPM) system for developing countries", Energy 2016; 109: 160-179

The remaining thesis' contributions are the comprehensive reviews of the global developments in photovoltaic, energy storage technologies, and the strategy for developing off-grid energy systems. These have also been published in the following journal articles:

(1) Akinyele D. O, Rayudu R. K, Nair N. K. C, "Global Progress in photovoltaic technologies and the scenario of solar panel plant and module performance estimation Application in Nigeria", Renewable and Sustainable Energy Reviews 2015; 48: 112-139.

(2) Akinyele D. O, Rayudu, "Review of energy storage technologies for sustainable power networks",Sustainable Energy Technologies and Assessments 2014; 8: 74-91.

(3) Akinyele D. O, Rayudu R. K, “Strategy for developing energy systems for remote communities: Insights to best practices and sustainability", Sustainable Energy Technologies and Assessments 2015; 16: 106-127.

\subsection{Future Work}

The thesis has presented a techno-economic and life cycle impact analysis of solar photovoltaic-based electrification systems for off-grid communities. Nigeria's six geo- 
political zones were used as case studies. The overall goal of the research is to develop new energy systems for those communities that do not have access to the national grid. The results have generally indicated that it is possible for remote communities to be electrified through well designed and planned solar photovoltaic technologies.

Renewable energy research, planning and development is not only wide, but also relatively new in many developing countries, including Nigeria. If the Sustainable Development Goal [224] target of electrifying every community of the world by the year 2030 is to be realised, both intensive and extensive research efforts will be necessary. This will be required to advance the renewable electricity applications in developing countries, especially for the remote and energy-poor communities. Based on this premise, the following future studies are recommended;

- To achieve the long-term viability of the energy systems, i.e. sustainability, a deepened social perspective must be integrated with the already considered technical-economic-environmental aspects. If localised electrical power systems based on renewable energies are to be developed, future research will be required to examine comprehensively the existing social structure and the people's consumption pattern. The author's practical experience and the familiarity with the communities and the energy consumption in buildings, used as case studies have been instrumental in developing a working load demand profile. This implies that a detailed survey of the existing appliances in the different households, the energy needs and the usage patterns is a requirement for determining the actual situation and making better decisions about off-grid SPMs development. Therefore, the use of data logger in future energy systems installation will help to record the users' electricity consumption. The recorded data will be useful for future design, research and analysis. This social aspect is an intrinsic part of the life of any energy project. It cuts across the pre-design, detailed design, implementation and post-implementation phases of energy system development. Since no energy project can survive without good institutional, policy, financial and management frameworks, the social perspective needs to be seriously considered as part of the future energy research. How the social aspect is quantified will constitute significant findings 
of such studies. Other financial metrics like return on investment and payback time need to be considered in the future work.

- Further studies will be needed on Integrated Solar Photovoltaic Microgrids (ISPMs) for multiple communities that are also expected to advance the solar power generation systems research through interconnected microgrids. Such research could make an interesting contribution in investigating the exchange of electrical power between the participating microgrids, the adequacy of energy and complementary characteristics. It can provide a path for reliable localised energy supply for zones or regions or communities in developing countries.

- The analysis of the thesis is based on different locations' solar energy resource. This allowed the author to ascertain how new electricity systems might be proposed for people who do not currently have access to energy supply in those locations. Such a study could be expanded in the future by investigating the possibility of hybridising more than one renewable energy resource, e.g. solar plus small hydro, solar plus biomass and solar plus wind. The research is expected to produce a broad range of techno-economic options toward $100 \%$ renewable electricity that can create more knowledge for planning and decisionmaking.

- Further studies on the effective energy management, control and coordination of the components of the off-grid energy solutions will also be required. Such research will provide the knowledge of the operation of off-grid renewable systems, which are relatively new in the power systems landscape.

- The introduction of dynamic and fault analyses of the solar electricity system to further research studies will also enrich the academic knowledge. This will help to achieve a better understanding of the behaviour of stand-alone electric power generation systems. Such contributions could be compared with the static analyses for the purpose of developing knowledge.

- Future research works that will present well-structured maintenance and monitoring procedures for solar photovoltaic energy systems for remote locations are also needed. The literature demonstrates that, while sizing and 
modelling of solar electricity systems are emphasised, there is little or no emphasis on the maintenance and monitoring aspects. Yet these constitute an important part of the post-implementation phase of the energy planning and development. The repair and/or replacement of the components of the solar photovoltaic plants must also be carefully considered. Further considerations on both corrective and preventive aspects of solar photovoltaic plant maintenance are expected to create useful information on how to promote renewable electricity applications. Research that incorporates the modelling of sensors, e.g. data logger with off-grid electricity systems could also provide good information about quantifying PV system performance. This could then be used as a basis for replicating such systems in another location, thus promoting renewable energy research and application.

- Off-grid microgrid solutions require sound financial and institutional frameworks because of their high initial capital investments. Research on effective business models for technical solutions could also provide a way of enriching the current body of knowledge.

- Research work also needs to be channelled towards educating the energy users on the most efficient way of using energy. The wide gap between how energy is used in the developed and the developing countries can generally be described by the level of awareness and education. The role of the research community in filling this gap cannot be over-emphasised. The education of the intended users will not only have a positive social impact, but will also help in conserving and managing the available energy.

\subsection{Closing Remarks}

This study has proposed Solar Photovoltaic Microgrids (SPMs) for different communities based on global engineering standards and best practices. It has presented a detailed techno-economic evaluation for the proposed energy systems, as well as the environmental impact analysis. It has also comprehensively presented the PV performance under different solar irradiance and ambient temperatures, leading to 
comparative evaluation of the proposed system's yields and losses in the specified locations.

Rigorous battery state of charge analysis is used to examine the solar energy's intermittent and seasonal variations. The study further presented a detailed reliability assessment for the proposed SPMs. This was done to ascertain the possible confidence level at which the energy systems can meet the users' energy consumption. The system reliability is quantified through the unmet load demand, loss of energy probability and the availability indices.

The results of the technical-economical-environmental analysis are comparable with those reported in the literature to evaluate the system's effectiveness, thus, contributing to the body of knowledge. The study has given general indications of how remote communities around the world, might be electrified through distributed photovoltaic power systems. It has also provided directions for future research studies to advance the academic knowledge and understanding of the application of solar energy in practical terms.

The proposed SPM model is based on global engineering standards and best practices and has very considerable practical applications. It can provide a reference point for researchers, designers, planners, policymakers, and other stakeholders of interest in conceptualising and proceeding with the design, planning and development of new electrification systems for remote communities. The research provides important information about the key energy design and planning indices, such as the users' energy needs and the electrical load patterns, the site characteristics, the possibility of load demand growth and the appropriate electricity generation capacity.

Solar PV-based microgrid options are progressively gaining recognition and relevance in the electricity industries for electrifying world's isolated and energy-poor communities. In other words, they are being identified as a key alternative for meeting the emerging countries' energy needs. This development is expected to continually open new opportunities that will lead to widespread use of solar technologies in those countries, in the not-too-far future. The findings set out in this thesis and the issues it raises could contribute to ensuring those opportunities are realised. 


\section{Bibliography}

[1] Rudnick H, Access to electricity: making the expansion worldwide, IEEE Power and Energy Mag. 2014; 12(4):20-25.

[2] Louie H, Dauenhauer P, Wilson M, Zomers A, Mutale J, Eternal light, IEEE Power and Energy Mag. 2014;12(4):70-78.

[3] Electricity access database - World Energy Outlook 2014.

[4] Akinyele D. O, Rayudu R. K, Distributed photovoltaic power generation for energypoor households: The Nigerian perspectives, In Proc. IEEE/PES Asia Pacific Power and Energy Engineering Conference (APPEEC), 2013, pp. 1-6.

[5] Akinyele D. O, Rayudu R. K, Nair N. K. C, Seah W. K. G, Clean development mechanism projects for developing countries: potential for carbon emissions mitigation and sustainable development, In Proc. $18^{\text {th }}$ National Power System Conference (NPSC), 2014, pp. 1-6.

[6] Akinyele D. O, Rayudu R. K, Nair N. K. C, Global progress in photovoltaic technologies and the scenario of solar panel plant and module performance estimation - application in Nigeria, Renewable and Sustainable Energy Reviews 2015; 48: 112-139.

[7] Akinyele D. O, Rayudu R. K, Nair N. K. C, Development of photovoltaic power plant for remote residential applications: the socio-technical and economic perspectives, Applied Energy 2015; 155: 131-149.

[8] Akinyele D. O, Rayudu R. K, Chakrabarti B, Nair N. K. C, Decentralized energy generation for end-use applications: the economic, social and environmental benefits assessment, In Proc. IEEE/PES Innovative Smart Grid Technologies (ISGT) Conference, 2014, pp. 1-4.

[9] Eight MDG Goals for 2015. Available: www.undp.org (accessed: 27/01/2015)

[10] Sustainable Energy for All. Available: www.se4all.org (accessed: 27/01/2015)

[11] Open working group proposal for Sustainable Development Goals. United Nations, 2014

[12] El Bassam N, Maegaard P, Schlichting M. L, "Distributed renewable energies for off-grid communities", 1st ed., Elsevier, 2013.

[13] Twidell J, Weir T, "Renewable energy resources", 2nd ed., Taylor and Francis eLibrary, 2006.

[14] Akinyele D. O, Rayudu, Review of energy storage technologies for sustainable power networks, Sustainable Energy Technologies and Assessments 2014; 8: 74-91.

[15] Freris L, Infield D, "Renewable energy in power systems", $1^{\text {st }}$ ed., John Wiley and Sons, 2008.

[16] Renewables 2015 - Global status report, REN21 2015; 1-251. 
[17] Enerdata- Global electricity statistical yearbook 2015.

[18] Masters GM. Renewable and efficient electric power systems. $1^{\text {st }}$ ed. John Wiley; 2004.p. 385-503.

[19] Luque A, Hegedus S. Handbook of photovoltaic science and engineering. $1^{\text {st }}$ ed. John Wiley; 2003. p. 154-204.

[20] El Chaar L, lamont LA, El Zein N. Review of photovoltaic technologies. Renewable and Sustainable Energy Reviews 2011; 15:2165-75.

[21] Moheimani NR, Parlevliet D. Sustainable solar energy conversion to chemical and electrical energy. Renewable and Sustainable Energy Reviews 2013;27: 494-04

[22] Yoo J, Cho J, Han K, Yi J. Solar cells fabrication on textured tricrystalline silicon wafers using a different wet texturing technique. Journal of Korean Physical Society 2011; 59:3047-50.

[23] Tyagi VV, Rahim NAA, Rahim NA, Selvaraj JAL. Progress in solar PV technology: research and achievement, Renewable and Sustainable Energy Reviews 2013; 20:443-61.

[24] Top 10 world's most efficient solar mono-crystalline PV modules 2012. Available: www.solarplaza.com (accessed: 15/01/2015)

[25] Manna TK, Mahajan SM. Nanotechnology in the development of photovoltaic cells. In Proc: International conference on clean energy power 2007. p. 379-86.

[26] SUNTECH 250W crystalline modules. Available: http://www.suntechpower.com/ (accessed: 14/01/2015)

[27] Satyen KD. Recent developments in high efficiency photovoltaic cells. Renewable Energy 1998; 15: 467-72.

[28] Iles PA. Evolution of space solar cells. Solar Energy Materials and Solar Cells 2001; 86:1-13.

[29] Akinyele DO. Passivation of crystalline silicon solar cells by thin amorphous layer. MSc dissertation, Centre for Renewable Energy Systems Technology (CREST), School of Electronic, Electrical and Systems Engineering, Loughborough University, UK, 2010.

[30] Schaper M, Schmidt J, Plagwitz H, Brendel R. 20.1\% Efficient crystalline silicon solar cell with amorphous silicon rear-surface passivation. Progress in Photovoltaics: Research and Applications 2005;13:381-86.

[31] Aberle AG. Crystalline silicon solar cells: advanced surface passivation and analysis. University of New South Wales 1999; 75: 130, 132.

[32] Staebler DL. Wronski CR. Reversible conductivity changes in dischargeproduced amorphous Si. Applied Physics Letters 1997; 31:292-4.

[33] Meier J, Spitznagel J, Kroll U, Bucher C, Fay S, Moriarty T, et al. Potential of amorphous and microcrystalline silicon solar cells. Thin Solid Films 2004; 451452:518-24. 
[34] Britt J. Ferekides C. Applied Physics Letters 1993; 62:2851.

[35] Dimova-Malinovska D. the state-of-the-art and furure development of the photovoltaic technologies - the route from crystalline to nanostructured and new emerging materials. Journal of Physics 2010; 253: 012007.

[36] Repins I, Conteras M, Egaas B, DeHart C, Scharf J, Perkins CL. 19.9\% efficient $\mathrm{ZnO} / \mathrm{CdS} / \mathrm{CuInGeSe} 2$ solar cell with $81.2 \%$ fill factor. Progress in Photovoltaics:Research and Applications 2008;16:235-9.

[37] Powalla M. The R and D potential of CIS thin-film solar modules. In Proc: $21^{\text {st }}$ European Photovoltaic Solar Energy Conference. 2006. p. 2137-41.

[38] Zhao J. Recent advances of high-efficiency single crystalline silicon solar cells in processing technologies and substrate materials. Solar Energy Materials and Solar Cells 2004; 82:53-64.

[39] Powalla M, Bonnet D. Thin film solar cells based on the polycrystalline compound semiconductors CIS and CdTe. Advanced Optoelectron 2007.p. 1-6.

[40] http://eu-solar.panasonic.net (accessed 12/02/2015)

[41] Wu CY, Matthews JA. Knowledge flows in the solar photovoltaic industry: insights from patenting by Taiwan, Korea and China. Research Policy 2012; 41:524-40.

[42] http://www.technosun.com/eu/downloads/SANYO-HIT-E01-series-datasheetEN.pdf (accessed: 10/01/2015)

[43] Kupich M, Grunsky D, Kumar P, Schroder B. Preparation of microcrystalline single junction and amorphous-microcrystalline tandem solar cells entirely by hotwire CVD. Solar Energy Materials and Solar Cells 2004; 81:141-6.

[44] Shah A, Meier J, Torres P, Kroll U, Fischer D, Beck N, et al. Recent Progress on microcrystalline solar cells. In Proc: $6^{\text {th }}$ IEEE Phtovoltaic Specilaists conference 1997.p. 569-74.

[45] Droz C, Vallat-Sauvain E, Bailat J, Feitknecht L, Meier J, Niquille X, et al. Electrical and microstructural characterization of microcrystalline silicon layers and solar cells. In Proc:3 ${ }^{\text {rd }}$ World Conference on Photovoltaic Energy Conversion 2003, p. 1544-7.

[46] Meier J, Kroll U, Vallat-sauvin E, Spitznagel J, Graf U, Shah A. Amorphous solar cells, the micromorphh concept and the role of VHF-GD deposition technique. Solar Energy 2004; 77:983-93.

[47] Chin-Yi T, Chin-Yao T. Development of amorphous/microcrystalline silicon tandem thin film solar modules with low output voltage, high energy yield, low light-induced degradation, and high damp-heat reliability. Journal of Nanomaterials 2014; 861742:1-10.

[48] Goetzberger A, Hebling C, Schock HW. Photovoltaic materials, history, status and outlook. Materials Science and Engineering R 2003; 40:1-46. 
[49] Deb SK, Ellingson R, Ferrere S, Frank AJ, Gregg BA, Nozik AJ, et al. Photochemical solar cells based on dye-sensitization of nanocrystalline $\mathrm{TiO}_{2}$. Photovoltaic Solar Energy Conversion 1998. NREL/VP-590-250-25056.

[50] Serrano E, Rus G, Garcia-Martinez J. Nanotechnology for sustainable energy. Renewable and Sustainable Energy Reviews 2009; 13:2372-84.

[51] Sethi VK, Pandey M, Shukla P. Use of nanotechnology in solar PV cell. International Journal of Chemical Engineering and Applications 2011;2(2):77-80.

[52] Arountiounian V, Petrosyan S, Khachatryan A. Studies of the photocurrent in quantum dot solar cells by the application of a new theoretical model. Solar Energy Materials and Solar Cells 2005; 89:165-73.

[53] Chen J, Zhao DW, Song Jl, Sun XW, Deng WQ, Liu XW, et al. Directly assembled CdSe quantum dots on $\mathrm{TiO}_{2}$ in aqueous solution by adjusting $\mathrm{pH}$ value for quantum dot sensitized solar cells. Electrochemistry Communications 2009; 11:2265-7.

[54] Gorji NE. A theoretical approach on the strain-induced dislocation effects in the quantum dot solar cells. Solar Energy 2012; 86:935-40.

[55] An assessment of solar energy conversion technologies and research opportunities. Global Climate and Energy Project, Stanford University 2006. p. 1-40.

[56] Akarslan F. Photovoltaic systems and applications, modeling and optimization of renewable energy systems, InTech 2012.

[57] Ghani F, Rosengarten G, Duke M, Carson JK. On the influence of temperature on crystalline silicon solar cell characterization parameters. Solar Energy 2015; 112:437445.

[58] Dally E. Life cycle cost analysis of a stand-alone PV system in rural Kenya. M.Sc dissertation, Purdue University, West Lafayette, Indiana, 2013.

[59] Brooks W, Dunlop J. Photovoltaic (PV) installer resource guide. North American Board of Certified Energy Practitioners, US 2014.

[60] Villalva MG, Gazoli JR, Filho ER. Comprehensive approach to modeling and simulation of photovoltaic arrays. IEEE Trans. Power Electronics 2009; 24:1198-208.

[61] Richter A, Hermlie M, Glunz SW. Reassessment of the limiting efficiency for crystalline silicon solar cells. IEEE Journal of Photovoltaics 2013; 3(4):1184-91.

[62] Mahmoud Y, Xiao W, Zeineldin HH. A simple approach to modeling and simulation of photovoltaic modules. IEEE Trans. Sustainable Energy 2012; 3(1):185-6.

[63] Single diode equivalent circuit model. PVPerformance modeling collaborative 2013 (accessed: 17/01/2015)

[64] Picault D, Raison B, Bacha S, de la Casa J, Aguilera J. Forecasting photovoltaic array power production subject to mismatch losses. Solar Energy 2010; 84:1301-9.

[65] Bandou F, Hadj Arab A, Belkaid MS. Test performance electrical of the photovoltaic module in two different environments. Energy Procedia 2013; 36:101824. 
[66] Hansen CW, Luketa-Hanlin A, Stein JS. Sensitivity of single diode models for photovoltaic modules to method used for parameter estimation. Sandia National Laboratories, US. p. 1-7.

[67] Tan RHG, Tan PLJ, Mok VH. Solar irradiance estimation based on photovoltaic module short circuit current measurement. In Proc: IEEE Int'l Conf. on Smart Instrumentation, Measurement and Applications 2013.p. 1-6.

[68] Tian H, Mancilla-David F, Ellis K, Muljadi E, Jenkins P. A cell-to-module-to-array detailed model for photovoltaic panels. Solar Energy 2012; 86(9):2695-706.

[69] Mayfield R. The highs and lows of photovoltaic system calculationsunderstanding calculations for proper system design and installation of photovoltaic systems, Electrical, Construction and Maintenance (ECM) 2012. p. 1-6.

[70] Denholm P, Ela E, Kirby B, Milligan M. The role of energy storage with renewable electricity generation. NREL Technical report, NREL/TP-6A2-47187, 2010.

[71] Chen H, Cong TN, Yang W, Tan C, Li Y, Ding Y. Progress in electrical energy storage system: A critical review. Progress Nat Science 2009; 19:291-312.

[72] Whittingham MS. History, evolution and future status of energy storage. Proceedings of the IEEE 2012; 100:1518-34.

[73] Electric energy storage systems. Quanta Technology, 2013. p. 1-12.

[74] DG ENER Working paper - The Future Role and Challenges of Energy Storage. European Commission Directorate-General for Energy, p. 1-36.

[75] Teleke S. Energy storage overview: applications, technologies and economical evaluation. White paper, Quanta Technology. p. 1-11.

[76] Electricity energy storage technology options, a white paper primer on applications, costs and benefits. Electric Power Research Institute, Report 1020676, 2010.

[77] Vazquez S, Lukic SM, Galvan E, Franquelo LG, Carrasco JM. Energy storage systems for transport and grid applications. IEEE Trans Ind Electron 2010; 57(12):3881-95.

[78] Handbook of Energy Storage for Transmission and distribution Applications. EPRI-DOE Report 1001834, 2003.

[79] Superconducting Magnetic Energy Storage Systems. Engineering and Technology news, 2011.

[80] Diaz- Gonzalez F, Sumper A, Gomis-Bellmunt O, Villafafila-Robles R. A review of energy storage technologies for wind power applications. Renew Sustain Energy Rev 2012; 16:2154-71.

[81] Bradbury K. Energy storage technology review, 2010. p. 1-33.

[82] Young-Min K, Jang-Hee L, Seok-Joon K, Daniel F. Potential and evolution of compressed air energy storage: energy and exergy analyses. Entropy 2012; 14:150121. 
[83] Lim S D, Mazzoleni A P, Park J, Ro P I, Quinlan B. Conceptual design of ocean compressed air energy storage system. In Proc: Oceans, 2012. p. 1-8.

[84] Advanced Adiabatic Compressed Air Energy Storage (AA-CAES). Energy Storage Association, 2013.

[85] ADELE- Adiabatic compressed -air energy storage for electricity supply. RWE Power, 2010.

[86] Isothermal CAES. Electricity Storage Association, 2014.

[87] Chen H, Zhang X, Liu J, Tan C. Compressed air energy storage. InTech, 2013;10112.

[88] Grazzini G, Milazzo. A thermodynamic analysis of multistage adiabatic CAES. In: Proceedings of the IEEE 2012; 100(2):461-72.

[89] Underwater compressed air energy storage: Islands and microgrids. White paper, Hydrostor, 2014. p. 1-13.

[90] Sub-surface pumped hydroelectric storage. Electricity Storage Association, 2014.

[91] Surface reservoir pumped hydroelectric storage. Electricity Storage Association, 2014.

[92] Pump up that seawater! A remix to pumped-storage hydro. Worldwatch Institute, 2012.

[93] Variable speed pumped hydroelectric storage. Electricity Storage Association, 2014.

[94] World's largest solar thermal plant with storage comes on-line. RenewEconomy, 2013.

[95] Pumped heat electrical storage (PHES). Electricity Storage Association, 2014.

[96] Howes J. Concept and development of a pumped heat electricity storage device. In: Proceedings of the IEEE 2012; 100(2):493-503.

[97] Kaldellis JK, Zafirakis D. Optimum energy storage techniques for the improvement of renewable energy sources-based electricity generation economic efficiency. Energy 2007; 32:2295-305

[98] Zinc-Bromine flow batteries. Electricity Storage Association, 2014.

[99] Acha S. Modelling distributed energy resources in energy service networks. Series 16. IET Renewable Energy; 2013.

[100] Burke AF. Batteries and ultracapacitors for electric, hybrid, and fuel cell vehicles. In: Proceedings of the IEEE 2007; 95(4):806 -20.

[101] Types of lead-acid batteries. Available: www.evbatterymonitoring.com (accessed: 25/09/2015)

[102] Advancements in lead-acid, Battery University 2015. Available: www.batteryuniversity.com/learn/article/advancements_in_lead_acid (accessed: 25/09/2015) 
[103] Adeoti O, Oyewole BA, Adegboyega TD, Solar photovoltaic-based electrification system for rural development in Nigeria: domestic load assessment, Renewable Energy 2001; 24: 155-161.

[104] Bugaje IM, Remote area power supply in Nigeria: the prospects of solar energy. Renewable Energy 1999; 18: 491-500.

[105] Oparaku OU, Photovoltaic systems for distributed power supply in Nigeria. Renewable Energy 2002; 25: 31-40.

[106] Oparaku OU, Rural area power supply in Nigeria: A cost comparison of the photovoltaic, diesel/gasoline generator and grid utility options. Renewable Energy 2003; 28: 2089-2098.

[107] Akpan US, Isihak SR, Udoakah YN. Electricity access in Nigeria: Viability of offgrid photovoltaic system. In Proc: IEEE AFRICON 2013, p.1-8.

[108] Oparaku OU. Assessment of the cost-effectiveness of photovoltaic systems for telecommunications in Nigeria. International Journal of Solar Energy 2002; 22(3-4): 123129.

[109] Charles A. How is $100 \%$ renewable energy possible for Nigeria? Global Energy Network Initiative 2014, p. 1-42.

[110] Olatomiwa LJ, Mekhilef S, Huda ASN. Optimal sizing of hybrid energy system for a remote telecom tower: A case study of Nigeria. In Proc: IEEE Conf. on Energy Conversion (CENCON) 2014, p. 243-247.

[111] Adaramola MS, Paul SS, Oyewola OM. Assessment of decentralized hybrid PV solar-diesel power system for applications in northern part of Nigeria. Energy for Sustainable Development 2014; 19: 72-82.

[112] Olatomiwa L, Mekhilef S, Huda ASN, Ohunakin OS. Economic evaluation of hybrid energy systems for rural electrification in six geo-political zones of Nigeria. Renewable Energy 2015; 83: 435-446.

[113] Cloutier M, Rowley P. The feasibility of renewable energy sources for pumping clean water in sub-Saharan Africa: A case study for central Nigeria. Renewable Energy 2011; 36: 2220-2226.

[114] Nfah EM, Ngundam JM, Vandenbergh M, Schmid J. Simulation of off-grid generation options for remote villages in Cameroon. Renewable Energy 2008; 33: 10641072.

[115] Bekele G, Boneya G. Design of a photovoltaic-wind hybrid power generation system for Ethiopian remote area. Energy Procedia 2012; 14: 1760-1765.

[116] Nfah EM, Ngundam JM. Feasibility of pico-hydro and photovoltaic hybrid power systems for remote villages in Cameroon. Renewable Energy 2009; 34: 14451450. 
[117] Chaurey A, Kandpal TC. Assessment and evaluation of PV based decentralized rural electrification: An overview. Renewable and Sustainable Energy Reviews 2010; 14:2266-2278.

[118] Yilmaz S, Ozcalik HR, Kesler S, Dincer F, Yelmen B. The analysis of different PV power systems for the determination of optimal PV panels and system installation A case study in Kahramanmaras, Turkey. Renewable and Sustainable Energy Reviews 2015; 52: 1015-1024.

[119] Ghafoor A, Munir A. Design and economics analysis of an off-grid PV system for household electrification. Renewable and Sustainable energy Reviews 2015; 42: 496-502.

[120] Rahman M, Barua S, Rahman M. Hybrid distributed renewable power generation for rural area: Bangladesh perspective. International Journal of Scientific and Engineering Research 2014; 5(10): 162-166.

[121] Akella AK, Sharma MP, Saini RP. Optimum utilization of renewable energy sources in a remote area. Renewable and Sustainable Energy Reviews 2007; 11: 894908.

[122] Dorji T, Urmee T, Jennings P. Options for off-grid electrification in the Kingdom of Bhutan. Renewable Energy 2015; 45: 51-58.

[123] Jamil M, Kirmani S, Rizwan M. Techno-economic feasibility analysis of solar photovoltaic power generation: A review. Smart Grid and Renewable Energy 2012; 3: 266-274.

[124] Bouzidi B. New sizing method of PV water pumping systems. Sustainable Energy Technologies and Assessments 2013; 4: 1-10.

[125] Jinayin T, Mungkung N, Kasayapanand N. Performance analysis of off-grid solar photovoltaic electrification systems for sustainable ICTs development: Field study in 4 Regions of Thailand. Energy Procedia 2014; 61: 1925-1928.

[126] Maleki A, Askarzadeh A. Optimal sizing of a PV/wind/diesel system with battery storage for electrification to an off-grid remote region: A case study of Rafsanjan. Sustainable Energy Technologies and Assessments 2014; 7: 147 - 153.

[127] Rehman S, El-Amin I. Performance evaluation of an off-grid photovoltaic system in Saudi Arabia. Energy 2012; 46: 451-458.

[128] Nandi SK, Ghosh HR. A wind-PV-battery hybrid power system at Sitakunda in Bangladesh. Energy Policy 2009; 37: 3659-3664.

[129] Laleman R, Albrecht J, Dewulf J. Comparing various indicators for the LCA of residential photovoltaic systems. Green Energy and Technology 2013: 211-239.

[130] Yue D, You F, Darling SB. Domestic and overseas manufacturing scenarios of silicon-based photovoltaics: Life cycle energy and environmental comparative analysis. Solar Energy 2014; 105: 669-678.

[131] Guide for array and battery sizing in stand-alone photovoltaic (PV) systems, IEEE Std. 1562, Dec. 2007. 
[132] Recommend practice for sizing lead-acid batteries for stand-alone photovoltaic (PV) systems, IEEE Std. 1013, Mar. 2007.

[133] Guide for selection, charging, test and evaluation of lead-acid batteries used in stand-alone photovoltaic (PV) systems, IEEE Std. 1361, Feb. 2004.

[134] Guide for optimizing the performance and life of lead-acid batteries in remote hybrid power systems, IEEE Std. 1561, Mar. 2007.

[135] Guide for test and evaluation of lead-acid batteries used in photovoltaic (PV) hybrid power systems, IEEE Std. 1661, Feb. 2008.

[136] Photovoltaic system performance monitoring- guidelines for measurement, data exchange and analysis, IEC 61724 Standard, 1998.

[137] Recommendations for small renewable energy and hybrid systems for rural electrification (Part 9-1: Micropower systems). IEC/TS 62257-9-1 standards, 2008.

[138] Recommendations for small renewable energy and hybrid systems for rural electrification (Part 2: From requirements to a range of electrification systems). IEC/TS 62257-2 standards, 2004.

[139] Recommendations for small renewable energy and hybrid systems for rural electrification (Part 4: System selection and design). IEC/TS 62257-4 standards, 2005.

[140] Recommendations for small renewable energy and hybrid systems for rural electrification (Part 7: Generators). IEC/TS 62257-7 standards, 2008.

[141] Recommendations for small renewable energy and hybrid systems for rural electrification (Part 7-1: Generators - Photovoltaic generators). IEC/TS 62257-7-1 standards, 2010.

[142] Recommendations for small renewable energy and hybrid systems for rural electrification (Part 7-3: Generator set - Selection of generator sets for rural electrification systems). IEC/TS 62257-7-3 standards, 2008.

[143] Solar photovoltaic energy systems - Terms, definitions and symbols. IEC/TS 61836 standards, 2007.

[144] Akinyele D. O, Rayudu R. K, "Strategy for Developing Energy Systems for Remote Communities: Insights to Best Practices and Sustainability", Sustainable Energy Technologies and Assessments 2016; 16: 106-127.

[145] Kumar A, Mohanty P, Palit D, Chaurey A, Approach for standardization of offgrid electrification projects, Renewable and Sustainable Energy Reviews 2009;13:19461956.

[146] Designing sustainable off-grid rural electrification projects: principles and practices. Operational guidance for World Bank Group Staff, The Energy and Mining Sector Board 2008.

[147] Arriaga M, Canizares CA, Kazerani M, Northern lights, IEEE Power and Energy Magazine 2014;12(4):50-59. 
[148] Mandelli S. Strategies for access to energy in developing countries: Methods and models for off-grid power systems design. Doctoral dissertation, Department of Energy, Politecnico Di Milano, 2014

[149] Saadat H. Power system analysis, $2^{\text {nd }}$ ed., McGraw-Hill, 2002 p. 8-13.

[150] Yearly average of daily sums of global horizontal irradiation of Nigeria. Available: www.sodais.com (accessed: 02/10/2014)

[151] Akinyele D. O, Rayudu R. K, Rodney H. G. Tan. Comparative Study of Photovoltaic Technologies Based on Performance, Cost and Space Requirement: Strategy for Selection and Application. International Journal of Green Energy 2015. DOI: $10.1080 / 15435075.2015 .1112287$

[152] HOMER index. Available: www.homerenergy.com

[153] Markvart T, Castaner L. Practical handbook of photovoltaics: fundamentals and applications, Elsevier 2003; 500-631.

[154] Tan RHG, Mok VH. A simplified approach for fundamental photovoltaic module performance analysis. In Proc: IEEE ISGT Asia 2014. p. 1-6.

[155] El Chaar L, lamont LA, El Zein N. Review of photovoltaic technologies. Renewable and Sustainable Energy Reviews 2011; 15:2165-75.

[156] Renewable energy off-grid renewable battery sizing. Trojan Battery Company 2013. Available: http://www.batterysizingcalculator.com/ (accessed: 01/10/2014)

[157] Freitas C. High-capacity battery banks. SOLARPRO 2012; 5.2: 1-6.

[158] Akinyele DO, Rayudu RK, "Techno-economic and environmental performance analyses of a solar photovoltaic microgrid (SPM) system for developing countries", Energy 2016.

[159] Khatib T., Mohamed A, Sopina K, Mahmoud M.: 'A new approach for optimal sizing of stand-alone photovoltaic systems', International Journal of Photoenergy, volume 2012, Article ID 391213, pp. 1-7.

[160] Gopi NP, Devendran S. Autonomy considerations for a standalone photovoltaic system', Sustainable Energy Technologies and Assessments 2015;10:79-83.

[161] Posadillo R., Lopez Luque R.: 'A sizing method for stand-alone PV installations with variable demand', Renewable Energy, 2008, 33, pp. 1049-1055.

[162] Bortolini M., Gamberi M., Graziani A.: 'Technical and economic design of photovoltaic and battery energy storage system', Energy Conversion and Management 2014; 86:81-92.

[163] DIgSILENT PowerFactory version 15 user manual- Technical references of model, 2014.

[164] Man EA. Control of grid connected PV systems with grid support functions. Master's thesis, Department of Energy Technology, Aalborg University, Denmark, 2012. 
[165] Ariyo FK, Omoigui MO. Investigation of Nigerian 330kV electrical network with distributed generation penetration - Part 1: Basic analyses. Electrical and Electronic Engineering 2013; 3(2): 49-71.

[166] Akinyele D. O, Rayudu R. K, Nair N. K. C, “Community-Based Hybrid Energy Supply System: A Practical and Comparative Approach", Applied Energy 2015. Accepted

[167] Guney I, Onat N. Cost calculation algorithm for photovoltaic systems: Paths to sustainable energy, InTech 2010. <www.intechopen.com>

[168] Xu L, Ruan X, Mao C, Zhang B, Luo Y. An improved optimal sizing method for wind-solar-battery hybrid power system. IEEE Transactions on Sustainable Energy 2013;4:774-85.

[169] Akinyele D. O, Rayudu R. K, Nair N. K. C. Grid-independent renewable electricity solutions for residential use: The case study of an off-grid house in Wellington, New Zealand. In Proc. IEEE/PES Asia Pacific Power and Energy Engineering Conference (APPEEC), 2015, pp. 1-5.

[170] World population prospects - key findings and advance tables. United Nations 2015; p.1-59.

[171] Seasons in Nigeria: North and South. Available: http://www.cometonigeria.com/about-nigeria/climate/ (accessed: 14/10/15).

[172] Brooks W, Dunlop J. Photovoltaic PV installer resource guide. North American Board of Certified Energy Practitioners (NABCEP) 2012, p. 1-161.

[173] Wenham SR, Green MA, Watt ME, Corkish R. Applied photovoltaics. $1^{\text {st }}$ ed. Earthscan UK; 2007. p. 1-195.

[174] Hodge BK. Alternative energy systems and applications. $1^{\text {st }}$ ed. John Wiley; 2010. p. $1-268$.

[175] Chakraborty S, Sadhu PK, Pal N. Technical mapping of solar PV for ISM - an approach toward green campus. Energy Science and Engineering 2015; 3(3):196-206.

[176] Duffie JA, Beckman WA. Solar engineering of thermal processes, $2^{\text {nd }} \mathrm{ed}$. Wiley NY; 1991.

[177] Gregg A, Paarker T, Swenson R. A "Real world" examination of PV system design and performance. In Proc. IEEE 31 ${ }^{\text {st }}$ Photovoltaic Specialists Conference 2005; $\mathrm{p}$. 1587-1592.

[178] Ayompe LM, Duffy A, McCormack SJ, Conlon M. Measured performance of a $1.72 \mathrm{~kW}$ rooftop grid connected photovoltaic system in Ireland. Energy Conversion and Management 2011; 52: 816-825.

[179] Huld TA, Suri M, Kenny RP, Dunlop ED. Estimating PV performance over a large geographical regions. In Proc. IEEE 31 ${ }^{\text {st }}$ Photovoltaic Specialists Conference 2005; p. $1679-1682$. 
[180] Ma T, Yang H, Lu L. Performance evaluation of a stand-alone photovoltaic system on an isolated island in Hong Kong. Applied Energy 2013; 112: 663-672.

[181] Mondol JD, Yohanis Y, Smyth M, Norton B. Long term performance analysis of a grid connected photovoltaic system in Northern Ireland. Energy Conversion and Management 2006; 47: 2925-2947.

[182] Jahn U et al. International Energy Agency task II database on photovoltaic power systems: statistical and analytical evaluation of PV operational data. In Proc. 15 th European Photovoltaic Solar Energy Conference, 1998. p.1-4.

[183] Sharma V, Chandel SS. Performance analysis of a $190 \mathrm{kWp}$ grid interactive solar photovoltaic power plant in India. Energy 2013; 55: 476-485.

[184] Padmavathi K, Daniel SA. Performance analysis of a 3MWp grid connected solar photovoltaic power plant in India. Energy for Sustainable Development 2013; 17: 615625.

[185] Lau KY, Yousof MFM, Arshad SNM, Anwari M, Yatim AHM. Performance analysis of hybrid photovoltaic/diesel energy system under Malaysian conditions. Energy 2010; 35:3245-3255.

[186] Tijani HO, Tan CW, Bashir N, Techno-economic analysis of hybrid photovoltaic/diesel/battery off-grid system in Northern Nigeria. Journal of Renewable and Sustainable Energy 2014; 6 (033103): 1-20.

[187] Faxas-Guzman J, Romero AJF, Roa-Escalante G, Tafur JP, Urbina A, Longer battery lifetime provided by a priority load control algorithm on stand-alone photovoltaic system. Journal of Renewable and Sustainable Energy 2015; 7 (013110): 120.

[188] Delavaripour H, Dehkordi BM, Reliability evaluation of a stand-alone windphotovoltaic/battery energy system based on realistic model of battery. Journal of Renewable and Sustainable Energy 2015; 7 (013107): 1-22.

[189] Salah CB, Lamamra K, Fatnassi A, New optimally technical sizing procedure of domestic photovoltaic panel/battery system. Journal of Renewable and Sustainable Energy 2015; 7 (013134): 1-13.

[190] Hasanuzzaman M, Al-Amin AQ, Khanam S, Hosenuzzaman M, Photovoltaic power generation and its economic and environmental future in Bangladesh. Journal of Renewable and Sustainable Energy 2015; 7 (013108): 1-12.

[191] Moharil RM, Kulkarni PS, A case study of solar photovoltaic power system at Sagardeep Island, India. Renewable and Sustainable Energy Reviews 2009; 13:673-681.

[192] www.vision-batt.com

[193] ETS hybrid charger and inverter- three phase. Available: www.jfy-tech.com accessed (10/10/2014) 
[194] Mohanty P, Muneer T, Kolhe M, Solar photovoltaic system applications: a guide for off-grid electrification. Green Energy and Technology, Springer eBook 2015. DOI 10.1007/978-3-319-14663-8.

[195] RETScreen International. Available: www.retscreen.net. accessed (19/12/2015)

[196] US Energy Information Administration 2015. Available: www.eia.gov/tools/faqs/faq.cfm?id=307\&t=11. Accessed (19/12/2015)

[197] Jakhrani AQ, Othman A, Rigit ARH, Samo SR, Estimation of carbon footprints from diesel generator emissions. In Proc. Intrenational Conf. in Green and Ubiquitous Technology 2012. p.78-81.

[198] Ito M, Life cycle assessment of PV systems - Crystalline silicon properties and uses. InTechopen 2011; 297-312.

[199] Fthenakis VM, Kim HC, Alsema E, Emissions from photovoltaic life cycles. Environmental Science and Technology 2008; 42(6):2168-2174.

[200] Jungbluth N, Life cycle assessment of crystalline photovoltaics in the Swiss ecoinvent database. Progress in Photovoltaics Research and Applications 2005; 13:429446.

[201] Alsema E. A, Energy pay-back time and $\mathrm{CO}_{2}$ emissions of PV systems. Progress in Photovoltaics Research and Applications 2000; 8:17-25.

[202] Olatomiwa L, Mekhilef S, Huda ASN, Sanusi K, Techno-economic analysis of hybrid PV-diesel-battery and PV-wind-diesel-battery power systems for mobile BTS: the way forward for rural development. Energy Science and Engineering 2015; 3(4): 271-285.

[203] Bortolini M, Gamberi M, Graziani A, Pilati F, Economic and environmental biobjective design of an off-grid photovoltaic-battery-diesel generator hybrid energy system. Energy Conversion and Management 2015; 106:1024-1038.

[204] Olatomiwa L, Mekhilef S, Ohunakin OS, Hybrid renewable power supply for rural health clinics (RHC) in six geo-political zones of Nigeria. Sustainable Energy Technologies and Assessments 2016; 13: 1-12.

[205] Ngan MS, Tan CW, Assessment of economic viability for PV/wind/diesel hybrid energy system in southern Peninsular Malaysia. Renewable and Sustainable Energy Reviews 2012;16(1):634-647.

[206] Campana PE, Li H, Yan J, Techno-economic feasibility of the irrigation system for the grassland and farmland conservation in China: Photovoltaic vs. wind power water pumping. Energy Conversion and Management 2015;103:311-320.

[207] Rezzouk H, Mellit A, Feasibility study and sensitivity analysis of a stand-alone photovoltaic-diesel-battery hybrid energy system in the north of Algeria. Renewable and Sustainable Energy Reviews 2015; 43:1134-1150.

[208] Sen R, Bhattacharyya SC, Off-grid electricity generation with renewable energy technologies in India: An application of HOMER. Renewable Energy 2014; 62:388-398. 
[209] Li C, Ge X, Zheng Y, Xu C, Ren Y, Song C, yang C, Techno-economic feasibility study of autonomous hybrid wind/PV/battery power system for a household in Urumqi, China. Energy 2013;55:263-272.

[210] Amutha WM, Rajini V. Techno-economic evaluation of various hybrid power systems for rural telecom. Renewable and Sustainable Energy Reviews 2015;43:553-561.

[211] Fthenakis V. How long does it take for photovoltaics to produce the energy used? The Magazine for Professional Engineers 2012; 16-17.

[212] Forster P, Ramaswamy V. Changes in atmospheric constitutents and in radiative forcing. In: Solomon S, Qin D, Manning M, Chen Z, Marquis M, Averyt K, Tignor MMB, Miller HL, Climate change 2007: The physical science basis. Contribution of working group I to IV assessment report of the IPCC. Cambridge University Press, UK.

[213] Akinyele DO, Rayudu RK. Comprehensive techno-economic and environmental impact analyses of a localized solar photovoltaic (PV) energy system for developing countries, Energy Conversion and Management 2016; 124: 266-279.

[214] Fthenakis V, Betita R, Shields M, Vinje R, Blunden J. Life cycle analysis of highperformance monocystalline silicon photovoltaic systems: Energy payback times and net energy production value. In Proc. 27 th European Photovoltaic Solar Energy Conference and Exhibition, 2012. pp. 4667-4672.

[215] Life cycle greenhouse gas emissions from electricity generation. National Renewable Energy Laboratory 2014. www.nrel.gov/analysis/sustain_ica_results.html (accessed: 20/04/16)

[216] PVSyst photovoltaic software. http://www.pvsyst.com/en/ (accessed: 12/12/15)

[217] Straw G. Understanding project management: Skills and insights for successful project delivery. $1^{\text {st }}$ Ed. Kogan Page Ltd., 2015. p. 71-80.

[218] Westland J. The project management life cycle: A complete step-by-step methodology for initiating, planning, executing and closing a project successfully. $1^{\text {st }}$ Ed. Kogan Page Ltd. , 2006. p. 90-128.

[219] Short W, Packey DJ, Holt T. A manual for the economic evaluation of energy efficiency and renewable energy technologies. National Renewable Energy Laboratory, NREL/TP-462-5173, 1995.

[220] NASENI solar energy limited. www.naseni.org (accessed: 10/02/2015)

[221] LV capacitor. www.argenelectric.com (accessed: 10/10/2014)

[222] Diesel generators. southwestproducts.com (accessed: 4/10/2014) 
[223] MicCom cable. www.micomcable.com (accessed: 11/10/2014)

[224] UN Sustainable Development Goals.

http://www.un.org/sustainabledevelopment/sustainable-development-goals/ (accessed: 11/10/2016)

[225] MAOT Consult Limited, Registered Engineers and Planners. Surulere, Lagos Nigeria

[226] Skoplaki E, Palyvos JA. Operating temperature of photovoltaic modules; A survey of pertinent correlations. Renewable Energy 2009; 34(1):23-29.

[227] Bardhi M, Grandi G, Tina GM. Comparison of PV cell temperature estimation by different thermal power exchange calculation methods. European Association for the Development of Renewable Energies, Environment and Power Quality (EA4EPQ), 2012.

[228] Denoix T, Sechilariu M, Locment F. Experimental comparison of photovoltaic panel operating cell temperature models. IEEE Industrial Electronics Society Conference 2014. 